Lars Otterpohl

\title{
Koordination in nichtlinearen dynamischen Systemen
}




\section{Lars Otterpohl}

\section{Koordination in nichtlinearen dynamischen Systemen}

Die Koordination der betrieblichen Teilbereiche ist im arbeitsteiligen System Unternehmung eine notwendige Voraussetzung für erfolgreiches Agieren am Markt. Die Arbeit hat zum Ziel, in der betrieblichen Praxis eingesetzte Koordinationsinstrumente auf ihre Wirkung in einem nichtlinearen, dynamischen Umfeld zu untersuchen. Hierzu wird eine Typologie für Koordinationsinstrumente verwendet und die einzelnen Koordinationstypen werden mit Hilfe der Spieltheorie und der Chaostheorie analysiert. Es zeigt sich, dass alle Typen grundsätzlich anfällig für deterministisches Chaos sind. Die systembestimmenden und damit Chaos auslösenden Parameter werden identifiziert und damit Maßnahmen aufgezeigt, wie chaotisches Verhalten verhindert werden kann.

Lars Otterpohl, geboren 1972 in Oberhausen, Studium der Wirtschaftswissenschaft an der RuhrUniversität Bochum; Abschluss zum Diplom-Ökonom 1996; Wissenschaftlicher Mitarbeiter am Lehrstuhl für Angewandte Betriebswirtschaftslehre I (Produktionswirtschaft) der Ruhr-Universität Bochum; Promotion zum Dr. rer. oec. 2001; seit August 2001 Referent im Controlling der Stinnes AG Mülheim. 
Koordination in nichtlinearen dynamischen Systemen 


\section{BOCHUMER BEITRÄGE ZUR UNTERNEHMUNGSFÜHRUNG UND UNTERNEHMENSFORSCHUNG}

Herausgegeben von Prof. Dr. Michael Abramovici,

Prof. Dr. Dr. h.c. mult. Walther Busse von Colbe, Prof. Dr. Dr. h.c. Wemer H. Engelhardt,

Prof. Dr. Roland Gabriel, Prof. Dr. Arno Jaeger, Prof. Dr. Gert Laßmann,

Prof. Dr. Wolfgang Maßberg, Prof. Dr. Bernhard Pellens, Prof. Dr. Marion Steven,

Prof. Dr. Rolf Wartmann, Prof. Dr. Brigitte Werners

Band 62

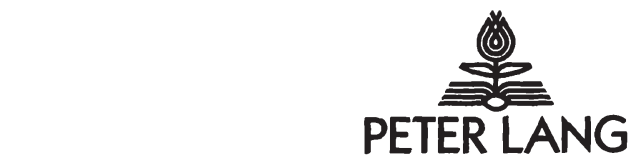

Frankfurt am Main - Berlin - Bern - Bruxelles - New York - Oxford - Wien 


\section{Lars Otterpohl}

\section{Koordination in nichtlinearen dynamischen Systemen}

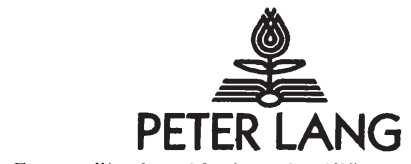

Europäischer Verlag der Wissenschaften 
Die Deutsche Bibliothek - CIP-Einheitsaufnahme

Otterpohl, Lars:

Koordination in nichtlinearen dynamischen Systemen / Lars Otterpohl. Frankfurt am Main ; Berlin ; Bern ; Bruxelles ; New York ; Oxford ; Wien : Lang, 2002

(Bochumer Beitrăge zur Unternehmungsführung und Unternehmensforschung ; Bd. 62)

Zugl.: Bochum, Univ., Diss., 2001

ISBN3-631-39214-1

Open Access: The online version of this publication is published on www. peterlang.com and www.econstor.eu under the international Creative Commons License CC-BY 4.0. Learn more on how you can use and share this work: http://creativecommons.org/licenses/by/4.0.

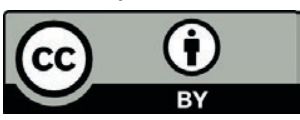

This book is available Open Access thanks to the kind support of ZBW Leibniz-Informationszentrum Wirtschaft.

Gedruckt auf alterungsbeständigem, säurefreiem Papier.

\author{
D294 \\ ISSN 0175-7105 \\ ISBN 3-631-39214-1 \\ ISBN 978-3-631-75508-2 (eBook) \\ (C) Peter Lang GmbH \\ Europäischer Verlag der Wissenschaften \\ Frankfurt am Main 2002 \\ Alle Rechte vorbehalten.
}

Das Werk einschließlich aller seiner Teile ist urheberrechtlich geschutzt. Jede Verwertung außerhalb der engen Grenzen des

Urheberrechtsgesetzes ist ohne Zustimmung des Verlages unzulässig und strafbar. Das gilt insbesondere für Vervielfältigungen, Übersetzungen, Mikroverfilmungen und die Einspeicherung und Verarbeitung in elektronischen Systemen.

Printed in Germany 123467 www.peterlang.de 
Für Yuet Ha

Lars Otterpohl - 978-3-631-75508-2 
Lars Otterpohl - 978-3-631-75508-2

Downloaded from PubFactory at 01/11/2019 04:01:56AM

via free access 


\section{Geleitwort}

Im arbeitsteiligen System Unternehmung ist die Koordination der verschiedenen betrieblichen Teilbereiche eine Aufgabe von grundsätzlicher Bedeutung. Die ökonomische Forschung hat sich bislang im Wesentlichen auf die Aufgabe konzentriert, Koordinationsmechanismen zu entwickeln und zu beschreiben. Modellbildungen zur Koordination sind meist vom Typ deterministischer linearer Modelle, sie untersuchen die im System vorhandenen Abhängigkeiten bei weitgehend konstanten Rahmenbedingungen. Dies wird der Komplexität der Realität mit ihren stochastischen Einflüssen und erratischen Schwankungen jedoch kaum gerecht. Herr Dr. Otterpohl hat sich die anspruchsvolle Aufgabe gestellt, in seiner nunmehr als Monographie vorliegenden Dissertation die Problemstellung der Koordination mit Hilfe chaostheoretischer Methoden zu untersuchen. Die Arbeit ist im Rahmen seiner Assistententätigkeit an meinem Lehrstuhl entstanden.

Die von Herrn Dr. Otterpohl untersuchte Problematik stellt in mehrfacher Hinsicht Neuland dar: Er untersucht die Koordination in Unternehmungen nicht nur mit dem Instrumentarium der Chaostheorie, sondern auch aus einem neuen Blickwinkel. Er behandelt die Frage, unter welchen Bedingungen der Einsatz von Koordinationsinstrumenten deterministisches Chaos auslösen kann und mit welchen Maßnahmen sich das Unternehmen wieder in stabile Bahnen lenken lässt. Die Untersuchung von Koordinationsmechanismen auf deterministisches Chaos erfordert die Anwendung formallogischer Modelle. Herr Dr. Otterpohl stellt zunächst die Lösungskonzepte der nichtkooperativen und der evolutionären Spieltheorie sowie deren Anwendung auf Koordinationsspiele vor und benutzt anschließend dieses Instrumentarium zur Analyse seiner Koordinationssituationen anhand von verschiedenen Modellen mit chaotischer Dynamik. Diese Modelle zeigen deutlich, dass eine Gleichgewichtsanalyse, wie sie in der Spieltheorie vorgenommen wird, nicht ausreicht, sondern dass die Forschung stärkeres Gewicht auf den zum Gleichgewicht führenden Pfad legen sollte. 
Die Ergebnisse der Arbeit stellen nicht nur einen theoretischen Erkenntnisfortschritt dar, sondern können durch das Aufzeigen von chaosanfälligen Strukturen auch die Anwendung von Koordinationsmechanismen in der betrieblichen Praxis verbessern. Die Arbeit nimmt eine geschlossene Diskussion der zugrunde gelegten Problemstellung vor und liefert darüber hinaus Ansatzpunkte für eine weiter führende Forschung auf dem Gebiet der Koordination in nichtlinearen dynamischen Systemen.

Prof. Dr. Marion Steven 


\section{Vorwort}

Unternehmen sind Systeme mit weit ausdifferenzierter Arbeitsteilung. Die Koordination der einzelnen Teilbereiche ist daher unabdingbare Voraussetzung eines erfolgreichen Agierens am Markt und seit jeher Gegenstand der Betriebswirtschaftslehre.

Die vorliegende Arbeit, die im Juli 2001 an der Ruhr-Universität Bochum als Dissertation angenommen wurde, rückt die nichtlineare Struktur betrieblicher Interdependenzen in den Mittelpunkt. Sie untersucht, wie sich Instrumente der Koordination verhalten, wenn sie in einem nichtlinearen, dynamischen Umfeld eingesetzt werden. Modelle, die dieses Szenario abbilden, sind nicht nur realitätsnäher als statische oder lineare Modelle, sondern sie können können im Unterschied zu jenen auch das Phänomen des deterministischen Chaos erklären. Als chaotisch bezeichnet man Systeme, die ein völlig regellos erscheinendes Verhalten zeigen und deren langfristige Entwicklung nicht prognostizierbar ist, obwohl sie keinerlei stochastischen Einflüssen unterliegen.

Solche Systeme lassen sich durch die Wahl geeigneter Werte für bestimmte Parameter in ruhige Bahnen lenken. Daher wird hier nicht nur das Ziel verfolgt, die Anfälligkeit von Koordinationsinstrumenten für deterministisches Chaos nachzuweisen, sondern auch die systembestimmenden Parameter und deren unkritische Wertebereiche zu identifizieren.

Die vorliegende Arbeit entstand während meiner Tätigkeit als wissenschaftlicher Mitarbeiter am Lehrstuhl für Produktionswirtschaft der RuhrUniversität Bochum. Sie wurde im Sommersemester 2001 von der Fakultät für Wirtschaftswissenschaft der Ruhr-Universität Bochum als Dissertation angenommen. Im Laufe ihrer Entstehung hat sich einige Dankesschuld angesammelt.

An erster Stelle möchte ich meiner akademischen Lehrerin Frau Prof. Dr. Marion Steven herzlich danken. Ihre wertvollen Anregungen, ihre stete Diskussionsbereitschaft und ihre intensive Betreuung haben ganz wesentlich 
zum Gelingen der Arbeit beigetragen. Weitere wichtige Hinweise verdanke ich Herrn Prof. Dr. Manfred Lösch, der das Korreferat übernommen hat.

Von zahlreichen konstruktiven Diskussionen und dem kritischen Lesen der Entwürfe hat die Arbeit sehr profitiert. Hierfür bin ich Frau Auguste Lamers, Herrn Dr. Sven Behrens, Herrn Dipl.-Kfm. Rolf Krüger und ganz besonders Herrn Dr. Peter Letmathe dankbar.

Mein größter Dank aber gilt meinen Eltern dafür, dass sie mir eine wissenschaftliche Ausbildung ermöglicht und mir immer zur Seite gestanden haben. Ohne meine Frau und ihre verständnisvolle Unterstützung wäre das vorliegende Buch sicherlich nicht zustande gekommen. Ihr widme ich daher diese Arbeit.

Lars Otterpohl 


\section{Inhaltsverzeichnis}

1 Einleitung. ..................... 1

2 Chaostheorie in den Realwissenschaften . . . . . . . . . 5

2.1 Chaostheorie in den Naturwissenschaften . . . . . . . . 10

2.2 Chaostheorie und Ökonomik . . . . . . . . . . . . . 12

2.2.1 Chaostheorie in der Volkswirtschaftslehre . . . . . . 13

2.2.2 Chaostheorie in der Betriebswirtschaftslehre . . . . . 20

3 Nichtlineare Dynamik . . . . . . . . . . . . . . . 27

3.1 Systemtheorie und Kybernetik . . . . . . . . . . . 28

3.2 Grundlegende Begriffe dynamischer Systeme . . . . . . . . 31

3.3 Topologisches Chaos . . . . . . . . . . . . . . 35

3.3.1 Die Definition nach Devaney . . . . . . . . . . 35

3.3.2 Chaos in der Sägezahnfunktion . . . . . . . . 38

3.3.3 Die Zeltfunktion . . . . . . . . . . . . . 40

3.3.4 Die logistische Parabel . . . . . . . . . . . . . . 43

3.3.5 Topologische Konjugation . . . . . . . . . . 46

3.4 Zur Numerik von Chaos . . . . . . . . . . . . 53

3.4.1 Das Beschattungslemma . . . . . . . . . . 53

3.4 .2 Lyapunov-Exponenten . . . . . . . . . . . . 57

3.4.3 Numerisches Chaos . . . . . . . . . . . . . . . . . 61 
3.5 Chaos in mehrdimensionalen Systemen . . . . . . . . . 62

3.5 .1 Grundlagen . . . . . . . . . . . . . 62

3.5.2 Exkurs 1: Lineare Modelle . . . . . . . . . . . . . . 62

3.5.2.1 Zeitdiskrete Modelle . . . . . . . . . . . 64

3.5.2.2 Zeitstetige Modelle . . . . . . . . . . 65

3.5.2.3 Linearisierung . . . . . . . . . . . 66 66

3.5.3 Exkurs 2: Zeitstetige Modelle der Dimensionen eins und zwei . . . . . . . . . . . . . . . 67

3.5.4 Definitionen . . . . . . . . . . . 70

3.5.5 Lyapunov-Spektrum . . . . . . . . . . . . 72

3.5.6 Fraktale Dimension . . . . . . . . . . . . . 75

3.5.7 Zwei seltsame Attraktoren . . . . . . . . . . . 82

3.6 Bifurkationstheorie . . . . . . . . . . . . . 85

3.6.1 Begriff der Bifurkation ............ 85

3.6.2 Sattelpunktbifurkation . . . . . . . . . . 89

3.6.3 Periodenverdopplung .............. 91

3.6.4 Hopf-Bifurkation . . . . . . . . . . . . . 92

3.6.5 Ein Musterbeispiel: Periodenverdopplung in der logistischen Parabel . . . . . . . . . . . . . . . 94

4 Koordination . . . . . . . . . . . . . . . . 99

4.1 Die Notwendigkeit der Koordination . . . . . . . . . . . . 99

4.1.1 Das ökonomische Verhaltensmodell . . . . . . . . . 99

4.1.2 Verhalten unter unvollständiger Information . . . . . 103

4.1 .3 Arbeitsteilung . . . . . . . . . . . . . . . 105

4.2 Sachliche und personelle Koordination . . . . . . . . . 107

4.3 Koordinationsformen . . . . . . . . . . . . . . . . . . 109

4.3.1 Koordinationstypologien in der Literatur . . . . . . . 110

4.3.2 Kritische Würdigung . . . . . . . . . . . 115 
5 Koordination und Chaos $\ldots \ldots \ldots \ldots \ldots$

5.1 Koordination in der Spieltheorie . . . . . . . . . 117

5.1.1 Strategische Form und Bimatrix-Form . . . . . . 118

5.1 .2 Lösungskonzepte . . . . . . . . . . . . . 120

5.1.2.1 Klassische Spieltheorie und Nash-Konzept . . 121

5.1.2.2 Kritik der klassischen Spieltheorie und evolutionäre Spieltheorie . . . . . . . . . . . 122

5.1.2.3 Evolutorische Ökonomik und evolutionäre Spieltheorie . . . . . . . . . . . 125

5.1 .3 Koordinationsspiele . . . . . . . . . . . . . . 128

5.1.3.1 Beispiele . . . . . . . . . . . . . 128

5.1.3.2 Normierung und Lösungen . . . . . . . . . 130

5.1.4 Ein konvergentes Koordinationsmodell . . . . . . 132

5.2 Koordination mit chaotischer Dynamik . . . . . . . . 133

5.2.1 Koordination durch Budgetierung . . . . . . . . 134

5.2.1.1 Modellformulierung. . . . . . . . . 134

5.2.1.2 Deterministisches Chaos . . . . . . . . 138

5.2.1.3 Stochastisches Chaos . . . . . . . . . . 143

5.2.2 Koordination durch Verrechnungspreise . . . . . . . 147

5.2.2.1 Die Organisationsstruktur: Innerbetriebliche Märkte . . . . . . . . . . 147

5.2.2.2 Das formale Modell . . . . . . . . . . . . . 149

5.2.2.3 Statische Eigenschaften: Allokationseffizienz . 153

5.2.2.4 Dynamische Eigenschaften: Chaotisches Tâtonnement . . . . . . . . . . . 157

5.2.3 Koordination durch Selbstabstimmung . . . . . . . 164

5.2.3.1 Das Szenario . . . . . . . . . . . 164

5.2.3.2 Die Elemente des Modells . . . . . . . . . 169 
5.2.3.3 Statische Eigenschaften: Nash-Gleichgewicht . 175

5.2.3.4 Dynamische Eigenschaften in kontinuierlicher Zeit: Stabilität . . . . . . . . . . . 179

5.2.3.5 Dynamische Eigenschaften in diskreter Zeit: eindimensionale Variante . . . . . . . . . . 182

5.2.3.6 Dynamische Eigenschaften in diskreter Zeit: zweidimensionale Variante . . . . . . . . . 188

5.3 Zusammenfassung der Ergebnisse . . . . . . . . . . 196

6 Fazit. . . . . . . . . . . . . . . . . . . 199

Literaturverzeichnis . . . . . . . . . . . . . . . . . . . 203 


\section{Abbildungsverzeichnis}

2.1 Konjunkturzyklen als Überlagerung harmonischer Wellen . . . 6

2.2 Steady State im Solow-Modell . . . . . . . . . . . . 15

2.3 Stabiler Steady State im Day-Modell . . . . . . . . . . . 17

2.4 Chaos im Day-Modell . . . . . . . . . . . . . . . . 17

2.5 Chaotische Zeitreihe im Day-Modell . . . . . . . . . . . . . 18

3.1 Klassifikation von Systemen . . . . . . . . . . . . 31

3.2 Grundform des kybernetischen Regelkreises . . . . . . . . . . 33

3.3 Sensitive Abhängigkeit von den Anfangsbedingungen . . . . 36

3.4 Topologische Transitivität . . . . . . . . . . . . 37

3.5 Dichte periodische Punkte . . . . . . . . . . . 38

3.6 Graph der Sägezahnfunktion . . . . . . . . . . . . . . . 39

3.7 Graph der Zeltfunktion . . . . . . . . . . . . . . . 41

3.8 Beschattungslemma . . . . . . . . . . . . . . . . 54

3.9 Lyapunov-Exponenten der logistischen Parabel . . . . . . . . 60

3.10 Diskrete und stetige Verhulst-Dynamik . . . . . . . . 68

3.11 Phasendiagramme zweidimensionaler Differentialgleichungssysteme . . . . . . . . . . . . . . . 6 69

3.12 Verformung einer Kreisscheibe . . . . . . . . . . . . . 72

3.13 Überdeckung einer Kurve durch Kreise . . . . . . . . . . 76

3.14 Konstruktion der Koch-Kurve . . . Lars Óterpo 
3.15 Hénon-Attraktor . . . . . . . . . . . . 83

3.16 Hénon-Attraktor - Detailansicht . . . . . . . . . . . . 83

3.17 Bahnen auf dem Hénon-Attraktor . . . . . . . . . . . . 84

3.18 Lorenz-Attraktor . . . . . . . . . . . . . . . . . . 85

3.19 Trajektorien auf dem Lorenz-Attraktor . . . . . . . . . . 86

3.20 Feigenbaum-Diagramm der logistischen Parabel . . . . . . . 87

3.21 Sattelpunktbifurkation (links) und Periodenverdopplung (rechts) 89

3.22 Phasendiagramm und Zeitreihe für die logistische Parabel . . . 95

3.23 Bifurkation in der logistischen Parabel . . . . . . . . . 96

4.1 Koordinationsinstrumente nach Schäffer . . . . . . . . . 111

4.2 Koordinationsinstrumente nach Kieser und Kubicek . . . . . . 111

4.3 Koordinationsinstrumente nach Laux und Liermann . . . . . . 113

4.4 Koordinationsinstrumente nach Schneider . . . . . . . . . 114

5.1 Pure Coordination $\ldots \ldots \ldots \ldots \ldots \ldots$

5.2 Win-Win-Game . . . . . . . . . . . . . . . . 129

5.3 Battle of Sexes . . . . . . . . . . . . . . . 129

5.4 Follow the Leader . . . . . . . . . . . . . . . . . . 131

5.5 Dangerous Coordination $\ldots \ldots \ldots \ldots \ldots \ldots$

5.6 Regelkreis des Modells $\mathcal{B} \ldots \ldots$. . . . . . . . . . 139

5.7 Phasendiagramme und Bahnen des deterministischen Budgetmodells für $n=\frac{1}{2}$ (oben) und $n=2$ (unten). . . . . . . 139

5.8 Feigenbaum-Diagramme des Modells $\mathcal{B}$, deterministische Variante, für $n=\frac{1}{2}$ und $n=2 \ldots \ldots \ldots 141$

5.9 Lyapunov-Exponenten des Modells $\mathcal{B}$, deterministische Variante, für $n=\frac{1}{2}$ und $n=2 \ldots \ldots \ldots \ldots 14 \ldots \ldots \ldots$

5.10 Feigenbaum-Diagramme des Modells $\mathcal{S}$, stochastische Variante, für $n=\frac{1}{2}$ und $n=2 \ldots \ldots \ldots \ldots \ldots$ 
5.11 Lyapunov-Exponenten des Modells $\mathcal{S}$, stochastische Variante, $n=\frac{1}{2}$ und $n=2 \ldots \ldots \ldots \ldots \ldots \ldots$

5.12 Struktur des produzierenden Systems $\mathcal{V} \ldots \ldots$. . . . . . 149

5.13 Technologien der liefernden (links) und der empfangenden (rechts) Stelle. . . . . . . . . . . . . . . . . . 150

5.14 Phasendiagramm und Bahn des Modells $\mathcal{V}$ für $\theta=0,85 \ldots 161$

5.15 Feigenbaum-Diagramm und Lyapunov-Exponenten des Modells $\mathcal{V}$ für $0 \leq \theta \leq \frac{8}{9} \ldots \ldots \ldots \ldots \ldots$

5.16 Feigenbaum-Diagramm und Lyapunov-Exponenten des Modells $\mathcal{V}$ für $0,8 \leq \theta \leq \frac{8}{9} \ldots \ldots \ldots \ldots \ldots$

5.17 Reaktionsfunktionen des Modells $\mathcal{S}$ für $\alpha=\beta \ldots \ldots 177$

5.18 Veränderungsrate und Differenz . . . . . . . . . . 180

5.19 Scheinbar doppelter Regelkreis der eindimensionalen Variante 182

5.20 Reaktionsfunktion $x_{t} \mapsto x_{t+2}$ für $\alpha=\beta=1 \ldots \ldots 183$

5.21 Phasendiagramme und Bahnen für $\alpha: \beta=1: 4$ (links) und $\alpha: \beta=1: 6,25$ (rechts) . . . . . . . . 185

5.22 Bifurkationsdiagramme des Modells $\mathcal{S}$, eindimensionale Variante186

5.23 Bahnen für Zyklen der Periode vier und acht . . . . . . . . 187

5.24 Lyapunov-Exponenten des Modells $\mathcal{S}$, eindimensionale Variante187

5.25 Phasendiagramm bei $\alpha / \beta=6,25$ nach 30.000 Iterationen $\ldots 188$

5.26 Echt doppelter Regelkreis der zweidimensionalen Variante . . . 190

5.27 Attraktoren im $x$ - $y$-Phasenraum für $\theta=0,94$ (oben links) bis $\theta=0,99$ (unten rechts) . . . . . . . . . . . 192

5.28 Lyapunov-Dimensionen des Modells $\mathcal{S}$, zweidimensionale Variante . . . . . . . . . . . . . . 193

5.29 Feigenbaum-Diagramme des Modells $\mathcal{S}$, zweidimensionale Variante . . . . . . . . . . . . . . . . . 194

5.30 Lyapunov-Exponenten des Modells $\mathcal{S}$, zweidimensionale Variante ....................... 195 
Lars Otterpohl - 978-3-631-75508-2

Downloaded from PubFactory at 01/11/2019 04:01:56AM

via free access 


\section{Kapitel 1}

\section{Einleitung}

Unternehmen sind soziotechnische Systeme von höchster Komplexität. Theorien zur Beschreibung, Erklärung und Gestaltung dieses Erkenntnisobjektes müssen abstrahieren, Wesentliches von Unwesentlichem trennen oder sich auf Einzelfragen konzentrieren. Die verschiedenen Teildisziplinen und Schulen der Ökonomik sind unterschiedliche Wege der Komplexitätsreduktion gegangen. Die Volkswirtschaftslehre reduziert Unternehmen in der Regel auf den güterwirtschaftlichen Bereich, indem sie nur die Input-Output-Beziehung in Form einer Produktionsfunktion verwendet. Das unternehmerische Verhalten wird durch das Gewinnmaximierungskalkül in Abhängigkeit von Beschaffungsund Absatzpreisen abgebildet. Die finanzwirtschaftliche Ebene wird ebenso ausgeblendet, wie Fragen des Rechnungswesens nicht erörtert werden. Die neoklassische Mikroökonomik, die durch das Wirken von Erich Gutenberg starken Einfluss auf die Betriebswirtschaftslehre genommen hat, ist überwiegend statisch formuliert, d.h. auf die Erörterung eines Gleichgewichtszustandes ausgerichtet, ohne dem Weg zum Gleichgewicht allzu viel Beachtung zu schenken.

Der funktionale Ansatz in der Betriebswirtschaftslehre versucht, der Komplexität in anderer Weise Herr zu werden, ohne grundlegende Fragen unternehmerischen Handelns ausschließen zu müssen. Er zerlegt das Unternehmen in Teilbereiche mit eigenen Aufgaben, insbesondere Beschaffung, Produktion, Absatz, Rechnungswesen, Finanzierung, Investition und Unternehmensführung. Die Unterteilung in Funktionen reduziert die Komplexität ganz erheblich, jedoch handelt man sich das Problem der Schnittstellenabstimmung ein. Die isolierte Betrachtung einer Teilfunktion vernachlässigt die Beziehungen zu anderen Teilfunktionen, die Koordination im Hinblick auf die Gesamtziele des Unternehmens wird erschwert. Der Versuch, dieses Problem durch To- 
talmodelle der Unternehmensplanung zu lösen, kann als gescheitert betrachtet werden; eine Zerlegung der Gesamtplanungsaufgabe ist unumgänglich. Hier können Modelle der hierarchischen oder modularen Planung eingesetzt werden.

Doch auch die Teilsysteme, wie z.B. die Produktion, sind noch so komplex, dass erhebliche Vereinfachungen nötig sind, um sie modellmäßig behandeln zu können. Häufig werden die Zusammenhänge zwischen den relevanten Variablen linearisiert. Auf diese Weise steht das Instrumentarium der Linearen Algebra und Optimierung zur Verfügung. Lineare Modelle zeigen in dynamischem Kontext aber nur wenige verschiedene Verhaltensmuster, nämlich Konvergenz, Divergenz und den Grenzfall dazwischen. Zeigt ein System nun erratische Schwankungen, muss als Erklärung auf stochastische Einflüsse zurückgegriffen werden. Sicher spielen solche exogenen Schocks eine erhebliche Rolle, doch zeigt die Untersuchung nichtlinearer Modelle, dass das System die Schwankungen auch aus sich selbst heraus generieren kann. Dies wird als deterministisches Chaos bezeichnet. Die Zeitreihen chaotischer Systeme sind, obgleich von deterministischen Bewegungsgesetzen erzeugt, von stochastischen Zeitreihen prima facie nicht zu unterscheiden. Die Beobachtung der Zeitreihe enthüllt keinerlei wiederkehrendes Muster. Eine Extrapolation einer solchen Zeitreihe in die Zukunft ist unmöglich. Hinzu kommt, dass selbst bei Kenntnis der Bewegungsgesetze eine langfristige Prognose des Systemverhaltens nicht möglich ist, weil unvermeidbare Messfehler bei der Bestimmung des gegenwärtigen Systemzustands die prognostizierte Zeitreihe von der tatsächlichen divergieren lassen.

In der vorliegenden Arbeit werden Modelle, die in der Praxis angewandte Koordinationsmechanismen abbilden, auf ihre Anfälligkeit für deterministisches Chaos untersucht. Die Modelle sind zwar in der Regel zu abstrakt, um unmittelbar in der Praxis anwendbar zu sein, dennoch muss die Unternehmensleitung, die für die Implementation von Koordinationsmechanismen verantwortlich ist, gegebenenfalls darauf achten, dass die Ursache von Schwankungen in den Mechanismen selbst liegen kann. Dass dies ganz andere Maßnahmen erfordert als die Abwehr exogener Schocks, liegt auf der Hand.

Der Gewinn, den die Betriebswirtschaftslehre aus der Anwendung chaostheoretischer Methoden ziehen kann, liegt also nicht darin, dass sie ein neues Paradigma erhält, das den Anspruch erhebt, an die Stelle linearer Modelle oder nichtmathematischer Methoden zu treten. Vielmehr vermittelt die Chaostheorie als Methode ergänzende Einsichten in die Ursachen unerwünschter und mit anderen Methoden nicht erklärbarer Phänomene, und mit diesen 
Einsichten lassen sich wirksame Handlungsempfehlungen zur Beseitigung jener Phänomene gewinnen.

Die Chaostheorie ist ein Teilgebiet der Mathematik, das in der Ökonomik bislang nicht zum Standardwerkzeug gehört. Daher gibt Kapitel 2 zunächst einen Überblick über die historische Entwicklung der Chaostheorie und ihre Anwendungen in den Realwissenschaften, insbesondere in der Volks- und Betriebswirtschaftslehre. In Kapitel 3 werden die wichtigsten Begriffe, Theoreme und Methoden vorgestellt. Die Darstellung ist rigoros mathematisch. Eine Verquickung mit ökonomischen Beispielen wird nicht vorgenommen, um dem an den mathematischen Hintergründen nicht interessierten Leser die Möglichkeit zu geben, dieses Kapitel nur gegebenenfalls beim Studium der Koordinationsmodelle zu Rate ziehen zu können, ohne den Gesamtzusammenhang $\mathrm{zu}$ verlieren. In Kapitel 4 wenden wir uns dem eigentlichen Untersuchungsgegenstand dieser Arbeit zu. Die Grundproblematik der Koordination wird erörtert und verschiedene Typologien von Koordinationsmechanismen werden vorgestellt. Diese Typologien geben ein Gerüst zur Einordnung der in Kapitel 5 entwickelten Modelle. Hier wird zunächst die Spieltheorie als Analysemethode und ein konvergentes Koordinationsmodell dargestellt, in dem die Koordination einem Fixpunkt zustrebt. Im Anschluss daran werden drei Modelle diskutiert, die zeigen, unter welchen Bedingungen unterschiedliche Koordinationsmechanismen anfällig für Chaos sein können, und welche die auslösenden Faktoren sind. Kapitel 6 fasst schließlich die Ergebnisse zusammen. Im Rahmen dieser Arbeit sind eine Reihe von Simulationsuntersuchungen durchgeführt worden. Die Ergebnisse und darauf beruhende Abbildungen hat, soweit nicht anders angegeben, der Autor durch eigene Programme mit Hilfe der Software „Mathematica Version 3.0“ von Wolfram Research erstellt. 
Lars Otterpohl - 978-3-631-75508-2

Downloaded from PubFactory at 01/11/2019 04:01:56AM

via free access 


\section{Kapitel 2}

\section{Chaostheorie in den Realwissenschaften}

Theorienbildung beruht in den Realwissenschaften auf dem Kausalitätsprinzip, mit dem induktive Schlüsse, die aus Einzelbeobachtungen allgemeine Gesetze ableiten, gerechtfertigt werden. Man unterscheidet zwei unterschiedlich strenge Ausprägungen des Prinzips. Nach der schwachen Kausalität haben gleiche Ursachen gleiche Wirkung. Dahinter verbirgt sich nichts anderes als die Erkenntnis, dass universell gültige Naturgesetze existieren, die dafür sorgen, dass zwei identische Ausgangszustände zum selben Endzustand führen. Ohne dies wären Prognosen und damit jede explikative oder gar normative Theorie unmöglich. Nun genügt die schwache Kausalität für die Bildung von ökonomischen Theorien in der Regel nicht, weil es höchstens unter Laborbedingungen gelingt, einen Ausgangszustand exakt zu reproduzieren. Naturwissenschaftliche Theorien hingegen machen von der experimentellen Methode intensiven Gebrauch. ${ }^{1}$

Die starke Kausalität geht einen Schritt weiter und behauptet, dass ähnliche Ursachen ähnliche Wirkung haben. Bei starker Kausalität wird der Geltungsbereich von Ursache-Wirkung-Zusammenhängen erheblich erweitert. Selbst wenn zwei Ausgangszustände nicht völlig identisch sind, kann man davon ausgehen, dass die Endzustände nah beieinander liegen. Eine Prognose auf der Grundlage des einen Ausgangszustands besitzt eine hinreichende Genauigkeit für den auf einem ähnlichen Ausgangszustand beruhenden tatsächlichen Endzustand.

1 In der Ökonomik fasst die Überprüfung von Theorien durch Laborexperimente seit den 60er Jahren langsam Fuß, ohne dass die experimentelle Methode bereits allgemein akzeptiert wäre. Vgl. hierzu Hey (1991). 


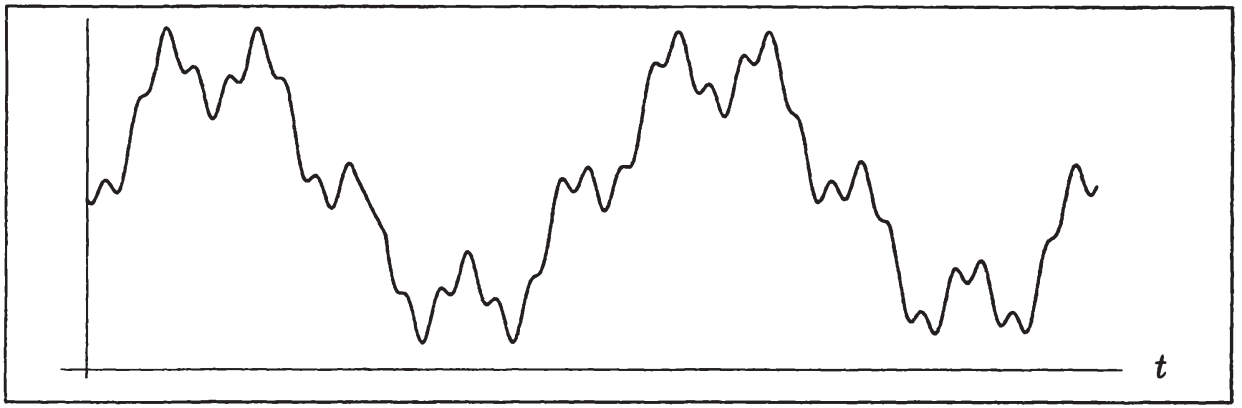

In Anlehnung an: Maußner (1994), S. 5

Abbildung 2.1: Konjunkturzyklen als Überlagerung harmonischer Wellen

Konsequent zu Ende gedacht entsteht aus dem Kausalitätsprinzip der Laplacesche Dämon, der in der Lage ist, aus der genauen Kenntnis ${ }^{2}$ von Ort und Impuls aller Teilchen zu einem Zeitpunkt das Schicksal des gesamten Universums in Vergangenheit und Zukunft zu berechnen; Ursache und Wirkung verbinden Ereignisse im Zeitablauf miteinander. Zufall ist demnach eine Illusion, die der Beschränktheit menschlicher Erkenntnisfähigkeit entspringt.

Die starke Kausalität ist das modellbildende Prinzip vor allem der inexakten Wissenschaften wie Soziologie, Ökologie oder Ökonomik. Diese Wissenschaften gehen nach wie vor überwiegend davon aus, dass eine annähernde Kenntnis der Anfangsbedingungen eine ungefähre Prognose des Systemverhaltens zulässt. Die beobachtbare Komplexität der Realität gilt als Überlagerung von vielen, für sich genommen jedoch einfach strukturierten Prozessen. Ein Musterbeispiel hierfür ist die empirische Konjunkturforschung, die lang-, mittel- und kurzfristige Zyklen zur Beschreibung von makroökonomischen Zeitreihen unterscheidet. Schumpeter bezeichnet diese Wellen nach ihren Entdeckern als Kondratieff-, Juglar- und Kitchin-Zyklen mit Perioden von ca. 50 Jahren, 10 Jahren und 3 Jahren. ${ }^{3}$ Abbildung 2.1 zeigt das Ergebnis einer Überlagerung von Sinuswellen mit diesen Perioden. In der statistischen Zeitreihenanalyse sind sogenannte Spektralschätzer entwickelt worden, d.h. Verfahren, deren Zweck die Analyse harmonischer Vorgänge und die Identifikation der Perioden sind. ${ }^{4}$

Zwei Revolutionen in der Physik zu Beginn des 20. Jahrhunderts erschütterten die Vorstellung von der Kausalität als Bindeglied zwischen Ereignis-

2 Damit wird lediglich schwache Kausalität angenommen.

3 Vgl. Schumpeter (1939), S. 161ff., sowie Maußner (1994), S. 3ff. und die dort angegebene Literatur.

4 Vgl. Schlittgen und Streitberg (1999), S. 353ff. 
sen nachhaltig. Erstens postulierte die allgemeine Relativitätstheorie, dass die Lichtgeschwindigkeit eine nicht zu überschreitende Obergrenze für die Fortbewegung ist. Das Hervorrufen einer wie auch immer gearteten Wirkung geschieht durch die Übertragung von Teilchen oder Wellen ${ }^{5}$, die sich maximal mit Lichtgeschwindigkeit bewegen. Für jede Ursache existiert ein raumzeitlicher Ereignishorizont, außerhalb dessen sie keine Wirkung hervorrufen kann. Im absoluten Anderswo kann ein Ereignis vom Hier-Jetzt aus weder beobachtet noch beeinflusst werden. ${ }^{6}$ Zweitens ist es in der Quantentheorie nach der Heisenberg-Unschärferelation unmöglich, Ort und Impuls eines Teilchens oder Zeitpunkt und Energie eines Vorgangs gleichzeitig beliebig genau zu bestimmen. In jedem Paar konjugierter Größen treten Unschärfen auf, deren Produkt nicht kleiner als ein Plancksches Wirkungsquant werden kann. ${ }^{7}$ Auf diese Weise sind im Makrokosmos und im Mikrokosmos der Erkenntnis unüberwindbare Grenzen gesetzt. Der Laplacesche Dämon wäre nicht in der Lage, Daten aus dem absoluten Anderswo zu sammeln, und ebenfalls nicht, die erreichbaren Daten ohne Unschärfe zu erlangen. ${ }^{8}$

Weder Relativität noch Unschärfe haben spürbare Auswirkungen auf den Mesokosmos, den Ort unserer unmittelbaren Anschauung. Um die Jahrhundertwende bemerkten nun einzelne Wissenschaftler, dass auch in deterministischen Systemen des Mesokosmos die Vorhersagbarkeit des Systemverhaltens eingeschränkt sein kann, wenn man die Anfangsbedingungen nicht mit absoluter Genauigkeit kennt. Der französische Mathematiker Henri Poincaré nahm viele Einsichten der Chaostheorie bereits um die Jahrhundertwende vorweg. Er beobachtete den Wechsel von laminaren und turbulenten Flüssen, untersuchte die Bewegungen dreier miteinander in Wechselwirkung stehender Körper und formulierte die Erkenntnis, dass kleine Unterschiede in den Anfangsbedingungen große Unterschiede in den späteren Erscheinungen hervorbringen. ${ }^{9}$ Der britische Physiker Maxwell vermutete, dass langfristige Wetterprognosen an lokalen Instabilitäten im Wettergeschehen scheitern. Auch erste Ansätze fraktaler Geometrie finden sich in dieser Zeit. KochKurve, Peano-Teufelstreppe, Sierpinski-Dreieck oder Menger-Schwamm galten allerdings als geometrische Kuriositäten ohne Bezug zur Realität, die

5 Nach der Theorie von der Welle-Teilchen-Dualität ist die Unterscheidung für Kausalitäten bedeutungslos. Vgl. Gerthsen (1997), S. 556ff.

6 Durch die beschränkte Lichtgeschwindigkeit ist es nicht möglich, ein Ereignis zu beobachten, das innerhalb des letzten Jahres in einer Entfernung von mehr als einem Lichtjahr stattgefunden hat. Vgl. Gerthsen (1997), S. 855.

7 Vgl. Gerthsen (1997), S. 896.

8 Streng genommen scheitert der Dämon schon daran, dass sein Datenspeicher größer als das Universum sein müsste.

9 Vgl. Poincaré $(1914,1973)$, S. 57. 
man verstanden zu haben glaubte und mit denen man sich nicht weiter beschäftigte. Die Zusammenhänge mit nichtlinearen Systemen und chaotischen Attraktoren entdeckte man erst viel später.

Der Beginn einer systematischen Chaosforschung wird gemeinhin auf das Jahr 1963 datiert. Der Meteorologe Edward Lorenz entdeckte in dem von ihm verwendeten Klimamodell sensitive Abhängigkeit von den Anfangsbedingungen, und er war der erste, dem die technischen Möglichkeiten zur Verfügung standen, dieses Phänomen systematisch zu untersuchen. Der LorenzAttraktor ${ }^{10}$ des Klimamodells ist heute zu einer Art Symbol der Chaosforschung geworden; dass es sich um einen sogenannten "Seltsamen Attraktor" handelt, wurde von Lorenz allerdings noch nicht erkannt. ${ }^{11}$ In einem weiteren Aufsatz stellte er die Frage, ob überhaupt ein Klima existiert. Mit anderen Worten, gibt es einen langfristigen Durchschnitt des Wetters? Die Analyse der Beobachtungswerte und seiner Klimamodelle ließ es möglich erscheinen, dass sich das Erdklima möglicherweise niemals auf ein Gleichgewicht mit durchschnittlichem Langzeitverhalten einpendelt. ${ }^{12}$

Etwa zur selben Zeit arbeitete der Mathematiker Steven Smale an nichtlinearen dynamischen Systemen aus topologischer Sicht. ${ }^{13}$ Er konnte nachweisen, dass auch strukturell stabile Systeme chaotisches Verhalten aufweisen können und entwickelte die „Smale-horseshoe-map“, eine Abbildung, die Chaos auf einen Mechanismus zurückführt, der sich als „Strecken und Falten" beschreiben lässt. ${ }^{14}$ Ein weiterer Meilenstein ist der Aufsatz von May ${ }^{15}$, einem Biologen, der die logistische Parabe ${ }^{16}$ als Modell einer Populationsentwicklung verwendete. Er untersuchte ihre Bifurkationen, d.h. die qualitativen Veränderungen ihres dynamischen Verhaltens in Abhängigkeit von Parameteränderungen ${ }^{17}$, hatte jedoch für den Übergang in chaotisches Verhalten noch keine rechte Erklärung. Sein wesentlicher Beitrag zur Chaostheorie besteht darin, breite Aufmerksamkeit auf das Problem der Periodenverdopplung gelenkt zu haben. Li und Yorke analysierten das Problem mit mathematischer Strenge und wiesen nach, dass ein dynamisches System, in dem

10 Vgl. Abschnitt 3.5.7.

11 Der Begriff ,Seltsamer Attraktor“ wurde geprägt in Ruelle und Takens (1971).

12 Vgl. Lorenz (1964).

13 Die Arbeiten Smales zu dynamischen Systemen sind zusammengefaßt in Smale (1980).

14 Vgl. Smale (1967). Das Strecken und Falten wird sehr ausführlich beschrieben in Peitgen, Jürgens und Saupe (1994), S. 72.

15 Vgl. May (1976).

16 Die logistische Parabel $f:[0,1] \rightarrow[0,1], x \mapsto a \cdot x(1-x)$ wird als Musterbeispiel in Form der Differenzengleichung $x_{t+1}=a \cdot x_{t}\left(1-x_{t}\right)$ in Kapitel 3 immer wieder herangezogen.

17 Den verschiedenen Formen von Bifurkationen widmet sich Abschnitt 3.6.5. 
ein Zyklus der Periode drei existiert, chaotische Eigenschaften besitzt. ${ }^{18} \mathrm{Da}-$ mit war der Begriff Chaos auch in die wissenschaftliche Diskussion eingeführt. Dieses Ergebnis war bereits ein Jahrzehnt zuvor von Sarkovskii entdeckt worden, jedoch in der westlichen Welt unbekannt geblieben. ${ }^{19}$ Das Theorem von $\mathrm{Li}$ und Yorke wird jedoch heute kaum mehr benutzt. Es ist nämlich nicht sicher gestellt, dass die Eigenschaften eines Systems, das diesem Theorem genügt, auch in der Realität bzw. Simulation beobachtbar sind.

In den siebziger Jahren dachte der amerikanische Physiker Feigenbaum über das Problem der Turbulenz nach in der Überzeugung, dass es seiner Wissenschaft nicht gelungen war, die wirklichen Probleme, nämlich die nichtlinearen, zu begreifen. In dem Bestreben, einen neuen Weg zu beschreiten, wandte er sich den einfachsten nichtlinearen Gleichungen zu, wobei er die realen komplexen Phänomene zunächst beiseite schob. Wie May bemerkte er, dass die Bifurkationen der logistischen Parabel dem Muster einer geometrischen Reihe folgen. Der Quotient zweier aufeinander folgender Parameterwerte, zu denen eine Bifurkation auftritt, konvergiert gegen 4,6692 ..., eine irrationale Zahl, von der heute noch nicht bekannt ist, ob sie transzendent ist ${ }^{20}$. Während May dieses Konvergenzverhalten für eine numerische Besonderheit hielt und umgehend wieder vergaß, ahnte Feigenbaum, dass die logistische Parabel eine Skalierung enthält ${ }^{21}$. Er erinnerte sich, dass man topologische Eigenschaften der logistischen Parabel entdeckt hatte, die auch in anderen Funktionen zu finden waren. ${ }^{22}$ Feigenbaum untersuchte die Differenzengleichung $x_{t+1}=a \sin \left(\pi x_{t}\right)$ und fand dasselbe Konvergenzverhältnis $4,6692 \ldots$ Inzwischen weiß man, dass diese Zahl, die sogenannte Feigenbaum-Konstante, für eine große Klasse von Systemen gilt, die auf den ersten Blick nichts miteinander zu tun haben. Die wichtigste Erkenntnis ist, dass Chaos keine völlige Regellosigkeit bedeutet, die sich in jeder Gleichung, in jedem realen Phänomen anders darstellt, sondern dass es eine verborgene Ordnung gibt, der man sich mit neuen Methoden und Perspektiven nähern kann. ${ }^{23}$

Die Chaosforschung gibt ein gutes Beispiel dafür, dass eine Idee nicht nur von einzelnen Wissenschaftlern entdeckt, sondern auch von der wissenschaftlichen Gemeinschaft gehört und verstanden werden muss. Für die Arbeiten

18 Vgl. Li und Yorke (1975).

19 Vgl. Sarkovskii (1964).

20 Transzendente Zahlen sind solche, die - im Gegensatz zu den ebenfalls irrationalen Wurzeln - nicht die Lösung einer Polynomialgleichung mit ganzzahligen Koeffizienten sind.

21 Inzwischen sind die Einzelheiten des Feigenbaum-Diagramms bekannt, und man kann in der Tat selbstähnliche Strukturen finden.

22 Vgl. Metropolis, Stein und Stein (1973).

${ }^{23}$ Die wichtigsten Aufsätze Feigenbaums sind Feigenbaum (1978) und Feigenbaum (1979). 
Poincarés war die Zeit noch nicht reif, statt dessen beschäftigte sich die theoretische Physik mehr als ein halbes Jahrhundert lang fast ausschließlich mit Relativität und Quantenmechanik. Lorenz publizierte seine bahnbrechenden Aufsätze im Journal of the Atmospheric Sciences und in einer schwedischen meteorologischen Fachzeitschrift namens Tellus ${ }^{24}$. Dort wurden sie von Wissenschaftlern, die ihrerseits chaotische Phänomene erforschten, natürlich mit erheblicher Verspätung zur Kenntnis genommen. Die mangelhafte Zusammenarbeit zwischen Forschern verschiedener Disziplinen und Kulturkreise verursachte eine nicht unbeträchtliche Doppelforschung.

\subsection{Chaostheorie in den Naturwissenschaften}

Die Chaosforschung hat ihren Ausgangspunkt in der Beobachtung von Komplexität in der Natur genommen. Die Strukturen von Wolken oder Pflanzen sind so kompliziert, dass eine Beschreibung mit den Begriffen der Euklidischen Geometrie unmöglich ist, doch blieb diese Komplexität lange unbeachtet. Man hatte die Vorstellung, dass sie durch die Überlagerung vieler, voneinander unabhängiger Strukturen entsteht. Ein inzwischen gut erforschtes Musterbeispiel ist die Turbulenz. Unter bestimmten Bedingungen fließt Wasser in einer stetigen und wirbelfreien Bewegung, meistens jedoch verwirbelt und diskontinuierlich, also turbulent. Laminare Fließbewegungen sind von der theoretischen Physik vollständig verstanden; Turbulenz, insbesondere der Übergang zwischen Ruhe und Turbulenz, widersetzte sich aber lange Zeit allen Erklärungsversuchen. Die Erklärung durch überlagernde Rhythmen steht jedenfalls nicht in Einklang mit der Beobachtung eines plötzlichen Übergangs in scheinbar regelloses Verhalten.

Ein weiteres physikalisches Beispiel chaotischen Verhaltens ist das anharmonische Pendel. Das Verhalten eines idealisierten, harmonischen Pendels ist vollkommen gleichförmig und vorhersagbar. Das anharmonische Pendel zeigt hingegen für einen bestimmten Bereich von Anfangsbedingungen Sensitivität, d.h. eine winzige Störung verursacht einen völlig anderen Verlauf der Pendelbewegungen.

24 Ein Physiker soll erbost ausgerufen haben: „Tellus! Kein Mensch liest Tellus.“ 
Das bekannteste Beispiel aus der Chemie ist die Belousov-ZhabotinskyReaktion $^{25}$,

$$
\begin{gathered}
\stackrel{\mathrm{Ce}^{3+} \stackrel{\mathrm{BrO}_{3}^{-}}{\longrightarrow}}{\longrightarrow} \mathrm{Ce}^{4+} \\
\mathrm{Ce}^{4+} \stackrel{\mathrm{C}_{3} \mathrm{H}_{3} \mathrm{BrO}_{4}}{\longrightarrow} \mathrm{Ce}^{3+}+\mathrm{Br}^{-}
\end{gathered}
$$

eine Redoxreaktion, die zwischen einem Überschuss von dreifach und vierfach geladenen Cer-Kationen hin- und herpendelt und dabei einen großen Reichtum an Erscheinungsformen zeigen kann. ${ }^{26}$ Die Idee der Selbstorganisation, d.h. der Entstehung komplexer Strukturen scheinbar aus dem Nichts, wie es sich etwa in Feigenbaum-Diagrammen ${ }^{27}$ beobachten lässt ${ }^{28}$, kann man anhand von chemischen Reaktionen demonstrieren. ${ }^{29}$

In der Medizin hat die Chaostheorie geholfen, das Problem der Herzrhythmusstörungen, das in Form des Kammernflimmerns zum Tode führen kann, auf eine wirksamere Weise anzugehen. ${ }^{30}$ Die Interpretation des Flimmerns als chaotisches Muster ermöglichte die Entwicklung zielgenauerer Defibrillatoren, wo man zuvor hinsichtlich der Stärke der nötigen Elektroschocks auf Vermutungen angewiesen war. Bei der Entwicklung künstlicher Herzklappen half die Erkenntnis, dass die Arbeit natürlicher Klappen das Volumen des Herzens in Form einer nichtlinearen Dynamik verändert. Die lineare Wirkung der ersten künstlichen Herzklappen barg die Gefahr einer Bildung von Blutgerinnseln, die Schlaganfälle auslösen können. ${ }^{31}$

Die Ökologie befindet sich hinsichtlich der Modellbildungsprobleme in einer ganz ähnlichen Situation wie die Ökonomik. Sie bedient sich des Instrumentariums der theoretischen Physik und behandelt ganze Populationen als dynamische Systeme, wobei von der Vielfalt in Flora und Fauna abstrahiert wird. Die realen Wirkungszusammenhänge sind so komplex, dass die mathematischen Modelle, deren Gleichungen aus empirischen Daten gewonnen werden, nur eine grobe Annäherung an die Wirklichkeit bieten können. Das

25 Vgl. Tomita und Tsuda (1979) und Tyson (1978).

26 Vgl. Ebeling (1991), S. 21.

27 Feigenbaum-Diagramme dienen der Veranschaulichung des Bifurkationsverhaltens dynamischer Systeme. Vgl. als Beispiel Abbilung 3.20.

28 Man denke an die dort zu findenden selbstähnlichen Strukturen und die Universalität der Feigenbaum-Konstante.

29 Mit diesem Gebiet eng verbunden ist der Name des belgischen Chemikers Ilya Prigogine. Vgl. z.B. Prigogine und Glansdorff (1971) und Prigogine, Allen und Herman (1977).

30 Vgl. Keener (1981) und Glass, Shrier und Bélair (1986).

31 Vgl. Garfield, Spano, Ditto und Weiss (1992). 
Erkenntnisinteresse gilt vornehmlich stabilen Lösungen. Produziert ein Modell erratisch schwankende Bahnen, wird in der Regel eine Fehlspezifikation der Modellgleichungen vermutet. Indes leben viele Populationen in Einjahresrhythmen mit nicht überlappenden Generationen, so dass sich die Populationsentwicklung am besten in Differenzengleichungen formulieren lässt. Die logistische Parabel beschreibt die Beschränktheit des Populationswachstums aufgrund knapper Ressourcen. Lange Zeit glaubte man, dass die regellos fluktuierenden Bahnen bei bestimmten Parameterwerten um einen verborgenen Gleichgewichtswert kreisen. Die Möglichkeit, es könne vielleicht gar kein Gleichgewicht geben, kam Ökologen überhaupt nicht in den Sinn. Erst May betrachtete ökologische Systeme aus der Sicht der Chaostheorie und fand, dass sich Daten über Polioepidemien oder Luchspopulationen durch nichtlineare Modelle erklären lassen. Sein Verdienst ist die Erkenntnis, dass die Chaos verursachenden Phänomene universalen Charakter tragen und von allen Wissenschaften zur Kenntnis genommen werden müssen. ${ }^{32}$

Die Anwendung der Chaostheorie ist in den Naturwissenschaften wesentlich weiter fortgeschritten als in den Geisteswissenschaften. Insofern trägt der vorstehende Abschnitt kursorische Züge und ist keineswegs vollständig; die Entwicklung schreitet in raschem Tempo voran. In neuerer Zeit versucht man, Chaos auch abseits des Mesokosmos zu entdecken. Eine Frage ist, ob die Bahnen der Planeten unseres Sonnensystems vielleicht in einer laminaren Phase sind, die irgendwann in eine chaotische übergehen wird. Außerdem wird untersucht, ob die hochgradig lineare Quantentheorie womöglich geändert werden muss, so dass ein "Quantenchaos" theoretisch erklärt werden kann.

\subsection{Chaostheorie und Ökonomik}

In den Sozialwissenschaften macht der Versuch einer Anwendung der Chaostheorie nur Sinn, wenn sich die Untersuchungsobjekte in mathematischer Sprache abbilden lassen. Daher beschränken sich chaostheoretische Ansätze auf diejenigen Bereiche der Wirtschaftswissenschaft, die sich hinsichtlich ihrer Methodik eng an die Physik anlehnen. Zwei der ganz wenigen chaostheoretischen Modelle außerhalb der Wirtschaftswissenschaft befassen sich mit den Bedingungen des Übergangs von politischen Spannungen in bewaffnete Konflikte. ${ }^{33}$ Ein frühes Modell über Verkehrsstaus kann man ungeachtet

32 May selbst nannte seinen Aufsatz in der Zeitischrift Nature einen „messianischen“ Artikel. Vgl. Gleick (1988), S. 120.

33 Vgl. Saperstein (1984) und Behrens, Feichtinger und Prskawetz (1997). 
der Publikation in einem physikalischen Journal im weiteren Sinne den Sozialwissenschaften zuordnen. ${ }^{34}$

Die bislang vorliegenden Arbeiten befassen sich zum einen mit der Formulierung theoretischer Modelle, die chaosgenerierende Mechanismen enthalten. ${ }^{35}$ Auf diese Weise soll fluktuierendes Verhalten ökonomischer Systeme ohne Rückgriff auf exogene Störungen beschrieben und erklärt werden. Zum anderen wird der Versuch unternommen, die aufgestellten Modelle mit der Realität zu konfrontieren. Dazu werden Zeitreihen ökonomischer Daten mit eigens dafür entwickelten statistischen Methoden auf die Existenz nichtlinearer Dynamik untersucht. Die Untersuchungen leiden jedoch durchweg an einer mangelnden Verfügbarkeit geeigneter Daten. Wenn überhaupt Daten zur Verfügung stehen, sind die vorliegenden Zeitreihen in der Regel zu kurz, um verlässliche Aussagen machen zu können. ${ }^{36}$ Daher hat sich in den letzten Jahren ein weiterer Zweig der empirischen Forschung etabliert: der Laborversuch. Hiermit können Modellimplikationen und Verhaltensannahmen unter kontrollierten Bedingungen überprüft werden, wobei die Experimente prinzipiell beliebig oft wiederholbar sind. ${ }^{37}$

\subsubsection{Chaostheorie in der Volkswirtschaftslehre}

Die Volkswirtschaftslehre nutzt die Erkenntnisse der Chaostheorie, um Fluktuationen in ökonomischen Zeitreihen zu erklären. Vor allem Konjunkturzy$k l e n$ lassen sich auf diese Weise modellieren, und es gibt eine Reihe von empirischen Untersuchungen, die versuchen, chaosgenerierende Mechanismen in Konjunkturdaten zu entdecken. Diese Ansätze verstehen sich als Gegenentwurf zu der bereits angerissenen Vorstellung von Konjunkturen als überlagernde harmonische Schwingungen. ${ }^{38}$

In seinem Beitrag zur Theorie des Wirtschaftswachstums aus dem Jahre 1956 entwarf Solow ein einfaches deskriptives Modell, das stabiles Wirtschaftswachstum auf Bevölkerungswachstum, Kapitalakkumulation und technischen Fortschritt zurückführt. ${ }^{39}$ Dieses Modell war über mehr als zwei

34 Vgl. Mushi und Higuchi (1976).

35 Vgl. die einführenden Übersichten Baumol und Benhabib (1989), Scheinkman (1990), Benhabib (1992) und Medio (1992).

36 Eine Ausnahme bilden Wertpapiermärkte, in denen täglich Preise ermittelt werden und die deshalb bei empirischen Untersuchungen gern herangezogen werden. Vgl. z.B. Elsner (1996) oder Thoma (1997).

37 Vgl. Sterman $(1988,1989)$.

38 Vgl. S. 6 f.

39 Vgl. Solow (1956). 
Jahrzehnte die Grundlage aller wachstumstheoretischen Forschung. 1982 zeigte Day, dass geringe Modifikationen der Modellannahmen schon genügen, um chaotisches Verhalten der modellendogenen Variablen zu erzeugen. Damit lieferte er zugleich eine modellimmanente Erklärung für Konjunkturzyklen. Das Solow-Modell sagt gleichmäßiges Wachstum voraus, Konjunkturschwankungen müssen über exogene Schocks erklärt werden. ${ }^{40}$

In der hier präsentierten Version ${ }^{41}$ des Solow-Modells wird die Zeit kontinuierlich gemessen, $t \in \mathbb{R}$. Eine geschlossene Volkswirtschaft produziert ein Gut $Y$, das sich sowohl zu Investitions- als auch zu Konsumzwecken eignet. Dazu werden die Produktionsfaktoren Arbeit $N$, Kapital $K$ und technisches Wissen $Z$ benötigt. Die Geltung des Sayschen Theorems ${ }^{42}$ wird angenommen, d.h. $Y, N$ und $K$ werden auf Märkten mit vollständiger Konkurrenz und flexiblen Preisen gehandelt, so dass die Märkte zu jedem Zeitpunkt geräumt sind. Daher genügt die Betrachtung der Angebotsseite der Volkswirtschaft, die Nachfrageseite ist nur implizit im Modell enthalten.

Die Bevölkerung und damit die Faktoreinsatzmenge $N(t)$ wächst mit einer konstanten Rate $n$ :

$$
N(t)=N_{0} \cdot e^{n \cdot t}
$$

Das technische Wissen wird mit der konstanten Rate $z$ akkumuliert:

$$
Z(t)=Z_{0} \cdot e^{z \cdot t}
$$

Technischer Fortschritt wirkt Harrod-neutral, so dass man Arbeit in Effizienzeinheiten messen kann: $Z \cdot N$. Anders ausgedrückt hat eine Vermehrung des technischen Wissens dieselbe Wirkung wie eine Erhöhung der eingesetzten Arbeit (in physischen Einheiten).

Kapital wird durch Konsumverzicht akkumuliert. Es wird angenommen, dass eine konstante Quote $s$ der Produktion gespart wird und eine konstante Rate $\delta$ des Kapitalstocks abgeschrieben wird. Damit ergibt sich der Zeitpfad der Kapitalstockentwicklung zu

$$
K(t)=\int_{0}^{t}(s \cdot Y(\tau)-\delta \cdot K(\tau)) d \tau+K_{0}
$$

Die gesamtwirtschaftliche Produktionsfunktion

$$
Y=F(Z \cdot N, K)
$$

40 Vgl. Maußner (1994), S. 41ff.

41 Vgl. Barro und Sala-i-Martin (1995), S. 16ff., und Maußner und Klump (1996), S. 36ff.

42 Nach dem Sayschen Theorem schafft sich jedes Angebot seine Nachfrage selbst, da niemand plant, etwas zu produzieren, der nicht plant, das entstehende Einkommen irgendwie zu verwenden. Vgl. Felderer und Homburg (1994), S. 84ff. 
ist zweimal stetig differenzierbar, linear-homogen und genügt dem Ertragsgesetz sowie den Inada-Bedingungen ${ }^{43}$. Daraus folgt, dass alle Produktionsfaktoren essenziell sind. Die weitere Analyse erfolgt anhand der ProKopf-Produktionsfunktion $y=f(k)$, die die Abhängigkeit der Pro-KopfProduktion $y=\frac{Y}{Z \cdot N}$ von der Pro-Kopf-Kapitalausstattung $k=\frac{K}{Z \cdot N}$ beschreibt $^{44}$. Sie hat die Eigenschaften:

$$
\begin{gathered}
f(0)=0, \quad f^{\prime}(k)>0, \quad f^{\prime \prime}(k)<0, \\
\lim _{k \rightarrow 0} f^{\prime}(k)=\infty, \quad \lim _{k \rightarrow \infty} f^{\prime}(k)=0 .
\end{gathered}
$$

Die Veränderungsrate der Pro-Kopf-Kapitalausstattung, die sogenannte fundamentale Gleichung der Kapitalakkumulation, ergibt sich aus den Annahmen zu

$$
\dot{k}=s \cdot f(k)-k(z+n-\delta) .
$$

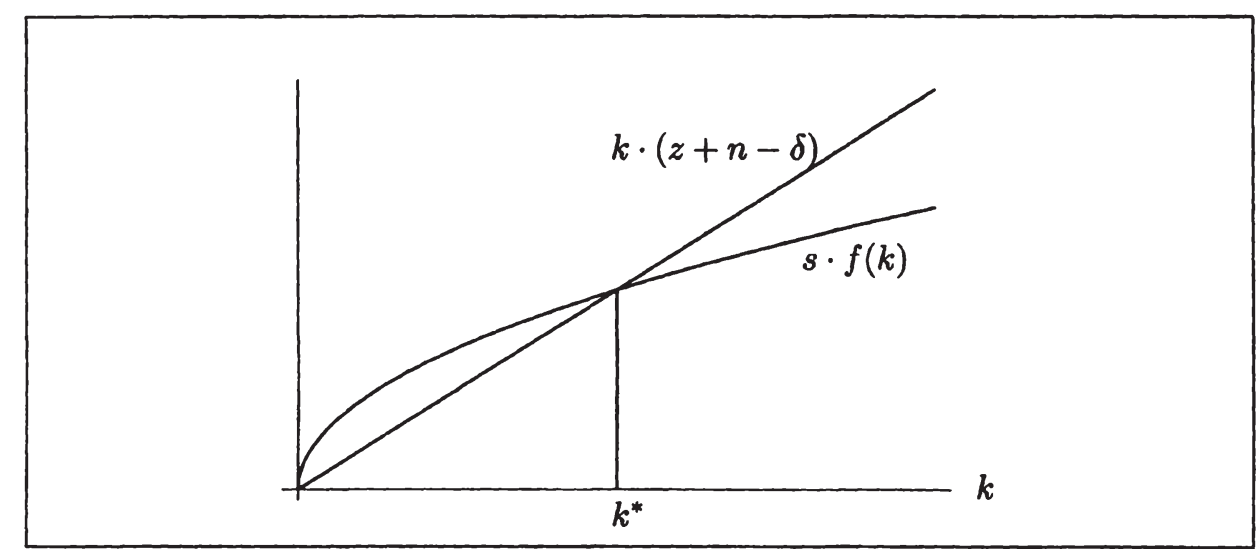

In Anlehnung an: Solow (1956), S. 70

\section{Abbildung 2.2: Steady State im Solow-Modell}

Ein Wachstumsgleichgewicht, auch Steady State genannt, ist erreicht, wenn alle Wachstumsraten konstant sind. Nun sind außer der Wachstumsrate der Kapitalakkumulation alle übrigen Wachstumsraten per Annahme

43 Nach den Inada-Bedingungen haben die Ableitungen $F_{K}$ und $F_{Z \cdot N}$ der Produktionsfunktion die Eigenschaften $\lim _{Z \cdot N \rightarrow 0} F_{Z \cdot N}=\lim _{K \rightarrow 0} F_{K}=\infty, \lim _{Z \cdot N \rightarrow \infty} F_{Z \cdot N}=$ $\lim _{K \rightarrow \infty} F_{K}=0$. Beispielsweise genügt die Cobb-Douglas-Produktionsfunktion $Y=$ $(Z \cdot N)^{\alpha} \cdot K^{1-\alpha}$ diesen Bedingungen.

44 Genau genommen müsste es Produktion bzw. Kapitalausstattung pro Einheit effizienter Arbeit heißen. 
konstant. Es bleibt als Gleichgewichtsbedingung $\dot{k}=0$, also nach der fundamentalen Gleichung

$$
s \cdot f(k)=k(z+n-\delta) .
$$

In Abbildung 2.2 sind die linke Seite der Gleichung (2.2) als Kurve und die rechte Seite als Gerade zu erkennen. Im Schnittpunkt befindet sich der Steady State, da die Gleichgewichtsbedingung (2.2) erfüllt ist. Er ist eindeutig, denn die Annahmen über die Pro-Kopf-Produktionsfunktion stellen sicher, dass nur ein Schnittpunkt existieren kann, und stabil, denn für $k<k^{*}$ gilt $\dot{k}>0$ und vice versa.

Im Solow-Modell wird der Steady State durch die neoklassische Produktionsfunktion sichergestellt. Dies gilt immer noch, wenn man wie Day die Zeit in diskreten Einheiten misst, $t \in \mathbb{Z}$. Die Wachstumsraten von Arbeit und technischem Wissen führen zu den Zeitpfaden

$$
N_{t}=N_{0}(1+n)^{t}
$$

bzw.

$$
Z_{t}=Z_{0}(1+z)^{t}
$$

Kapitalakkumulation vollzieht sich nach der Gleichung

$$
K_{t}=\sum_{\tau=0}^{t}\left(s \cdot Y_{\tau}-\delta K_{\tau}\right)+K_{0} .
$$

Die fundamentale Gleichung in Form einer Differenzengleichung $k_{t+1}=$ $\psi\left(k_{t}\right)$ ist analog zu Gleichung (2.1) und lautet

$$
k_{t+1}=\frac{s \cdot f\left(k_{t}\right)}{(1+z)(1+n)}+\frac{(1-\delta) k_{t}}{(1+z)(1+n)} .
$$

Das Phasendiagramm 2.3 zeigt die Kurve zu Gleichung (2.3). Im Schnittpunkt dieser Kurve mit der Gleichgewichtsbedingung $k_{t+1}=k_{t}$ findet sich wiederum ein eindeutiger und stabiler Steady State mit $k^{*}=\psi\left(k^{*}\right)$.

Day nimmt eine zweite Änderung vor. Er ersetzt die neoklassische ProKopf-Produktionsfunktion durch

$$
\phi(k)=b \cdot k^{\beta}(m-k)^{\gamma}, \quad b, \beta, m, \gamma>0
$$

und begründet dies mit möglichen negativen Rückwirkungen der Produktion. Es fallen Schadstoffe an, die in geringen Mengen von der Umwelt abgebaut 


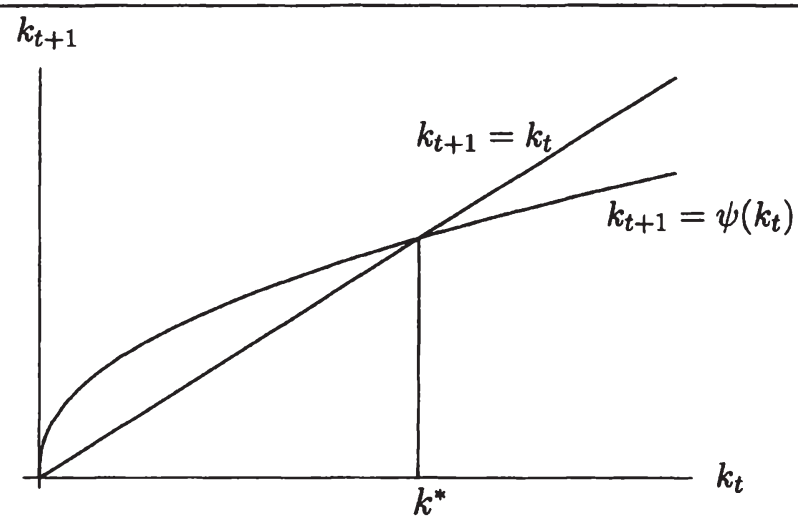

In Anlehnung an: Day (1982), S. 410

Abbildung 2.3: Stabiler Steady State im Day-Modell

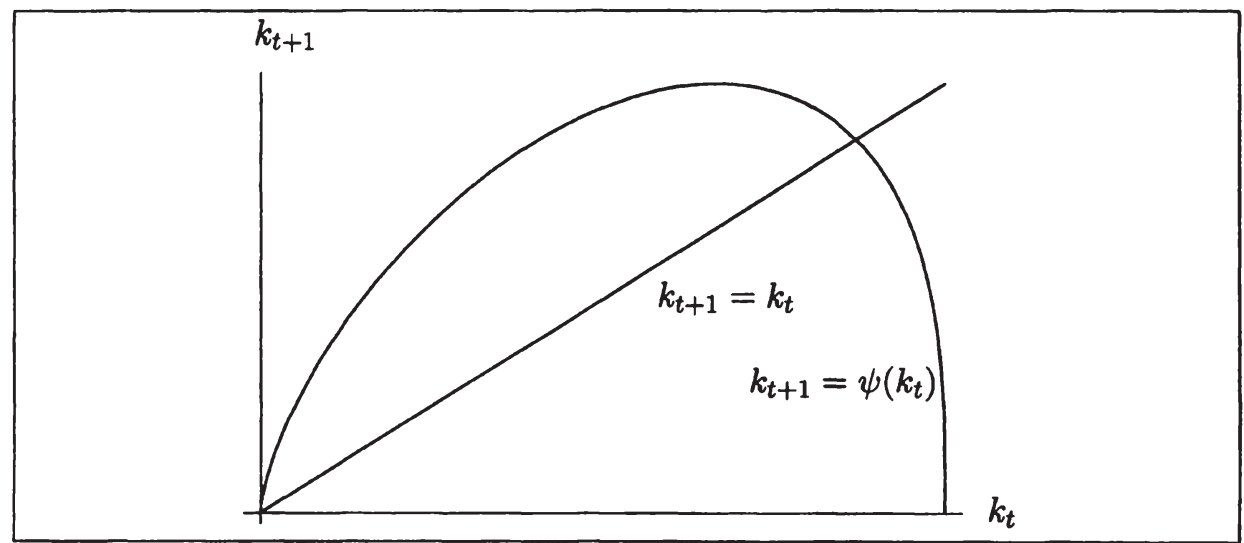

In Anlehnung an: Day (1982), S. 410

Abbildung 2.4: Chaos im Day-Modell

werden können, bei steigender Kapitalintensivierung aber die Produktion beeinträchtigen. Die Produktionsmenge kann nicht unbegrenzt gesteigert werden, sondern erreicht bei $k^{\max }=\frac{\beta \cdot m}{\beta+\gamma}$ ihr Maximum. Setzt man $\phi$ in die fundamentale Gleichung (2.3) ein und wählt die Parameter $\delta=m=\beta=\gamma=1$, so erhält man die logistische Parabel..$^{45}$ Für $\beta, \gamma \neq 1$ ergibt sich eine unimodale Funktion, die in Abbildung 2.4 dargestellt ist.

$\overline{45}$ Die Wahl $m=1$ bedeutet nur eine Normierung. Die Abschreibungsrate $\delta=1$ zu setzen, heißt $\phi$ als Bruttoproduktionsfunktion zu interpretieren. 


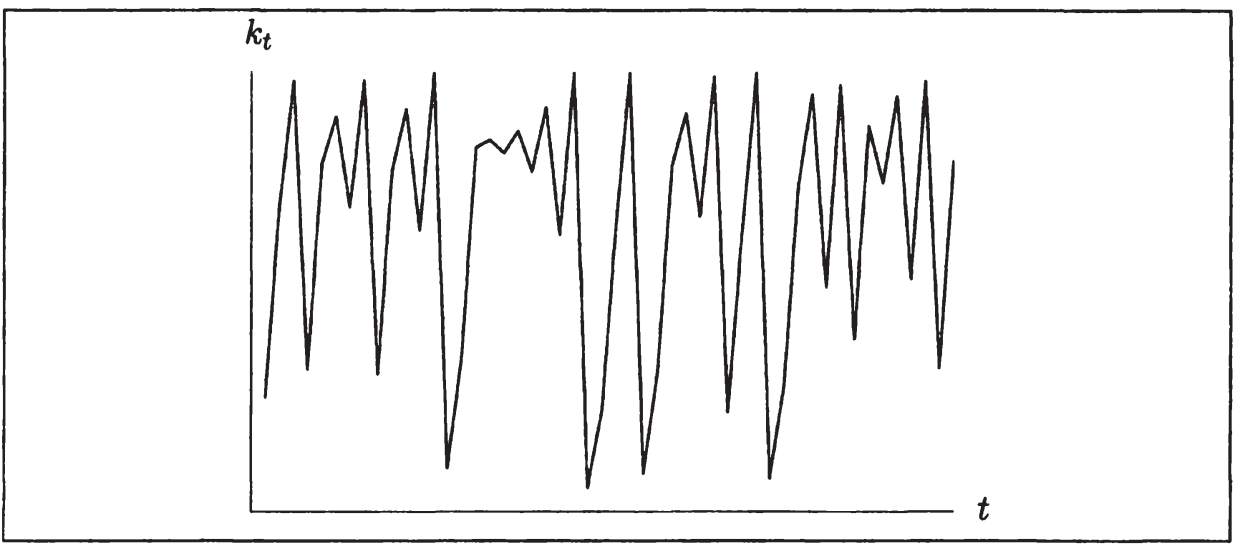

Abbildung 2.5: Chaotische Zeitreihe im Day-Modell

Auch hier zeigt sich ein Steady State im Schnittpunkt von Winkelhalbierender und dem Graphen der Funktion $\psi$. Jedoch weist dieser Steady State keine Stabilität auf, weil die Steigung von $\psi$ im Schnittpunkt betragsmäßig größer als Eins ist. Vielmehr oszilliert die Zeitreihe der Pro-KopfKapitalausstattung $k_{t}$ und damit auch der Pro-Kopf-Produktion $y_{t}$ scheinbar regellos im Intervall $(0, m)$, obwohl das Modell keine stochastischen Variablen enthält. Abbildung 2.5 zeigt eine solche chaotische Zeitreihe. ${ }^{46}$

Die Volkswirtschaftstheorie, die sich häufig der Methoden der Naturwissenschaften, vor allem der Physik bedient, hat auch die Chaostheorie in einer Fülle von Modellen intensiv genutzt. Eine Reihe von Aufsätzen variiert den Overlapping-Generations-(OLG-)Kontext, um endogene Aperiodizität zu modellieren. OLG-Modelle sind dadurch gekennzeichnet, dass die Individuen bei diskreter Zeitmessung zwei Perioden lang leben, so dass in jeder Periode eine junge und eine alte Generation existiert. ${ }^{47}$ Das Modell von Grandmont erklärt Konjunkturzyklen in einem Szenario vollkommener Märkte, die permanent geräumt sind, bei vollkommener Voraussicht der Marktteilnehmer. Exogene Schocks, insbesondere staatliche Eingriffe, werden außen vor gelassen. Der Ursprung der Zyklen liegt im Konflikt zwischen intertemporalem Einkommens- und Substitutionseffekt. ${ }^{48}$ In anderen OLG-Modellen entsteht Chaos durch positive Abhängigkeit der Diskontrate vom Vermögen der Indi-

46 Die mathematischen Hintergründe für ein solches Systemverhalten werden ausführlich im folgenden Kapitel dargelegt.

47 Die Methode der Overlapping Generations geht zurück auf Diamond (1965).

48 Vgl. Grandmont (1985). 
viduen ${ }^{49}$ bzw. intergenerationalen Handel bei bestimmten Eigenschaften der Nutzenfunktionen ${ }^{50}$.

Sogar bei optimierendem Verhalten der Individuen sind chaotische Pfade möglich. Aufbauend auf einem Modell von Benhabib und Nishimura, das robuste zweiperiodische Zyklen unter Standardannahmen für eine ZweiSektoren-Ökonomie nachweist ${ }^{51}$, untersuchen Deneckere und Pelikan, unter welchen Bedingungen bezüglich Technologie und Präferenzen Chaos auftritt $^{52}$. Boldrin und Montrucchio zeigen, dass in mehrsektoralen Ökonomien bei hinreichend kleiner Diskontrate jede Politikfunktion mit optimierendem Verhalten vereinbar ist, also auch solche, die chaotische Zeitpfade verursachen. ${ }^{53}$ Diese wichtige Erkenntnis, dass rationales, optimierendes Verhalten zu Chaos führen kann, wurde von Kopel und Feichtinger auf betriebswirtschaftliche Fragestellungen übertragen. ${ }^{54}$

In die Mikroökonomik gehören Konsumwahlmodelle, die die Nachfrage nach einzelnen Gütern zum Gegenstand haben. Bei Benhabib und Day ergibt sich Chaos aus der Abhängigkeit der Präferenzen von den bisherigen Erfahrungen und dem Verlangen nach Abwechslung, also zyklischen Präferenzen. Damit wird die sonst übliche Annahme stabiler Präferenzen bei veränderlichen Restriktionen fallen gelassen. ${ }^{55}$ In zwei einander recht ähnlichen Modellen entsteht Chaos durch interpersonelle Effekte der Konsumwahl. Kaufentscheidungen hängen von den Entscheidungen anderer Individuen ab, was in der Literatur als Bandwagon-Effekt bekannt ist, aber selten explizit analysiert wird. ${ }^{56}$ Bei Gaertner berücksichtigen die Akteure die Erfahrungen ihrer „Nachbarn "57, bei Granovetter und Soong hängt die Kaufentscheidung von der in der Vergangenheit von anderen Individuen gekauften Menge eines Gutes $\mathrm{ab}^{58}$.

\footnotetext{
Vgl. Benhabib und Day (1980).

Vgl. Benhabib und Day (1982).

Vgl. Benhabib und Nishimura (1985).

Vgl. Deneckere und Pelikan (1986).

3 Vl. Boldrin und Montrucchio (1986).

54 Vgl. Kopel und Feichtinger (1995).

55 Vgl. Benhabib und Day (1981).

56 Eine chaostheoretische Analyse liefern Feichtinger, Hommes und Milik (1997).

57 Vgl. Gaertner (1986).

58 Vgl. Granovetter und Soong (1986).
} 


\subsubsection{Chaostheorie in der Betriebswirtschaftslehre}

In der Betriebswirtschaftslehre gibt es vergleichsweise wenige chaostheoretische Ansätze ${ }^{59}$, bislang sind solche Methoden in der Betriebswirtschaftslehre nicht allgemein akzeptiert. Daher wird im Folgenden begründet, warum die Chaostheorie Erkenntnisse bei betriebswirtschaftlichen Fragestellungen erwarten lässt. Lassen sich Mechanismen finden, die einerseits in der Chaostheorie verwendet werden, andererseits aber auch bei betriebswirtschaftlichen Fragestellungen zum Einsatz kommen können, ist eine Anwendung der Chaostheorie gerechtfertigt. ${ }^{60}$

Die Ausführungen in Kapitel 3 werden zeigen, dass Chaos zwei Systemeigenschaften voraussetzt. Zum einen müssen die Systemgleichungen nichtlinear sein, und zum anderen muss es sich um ein rückgekoppeltes System handeln, d.h. die Systemvariablen müssen Funktionen ihrer eigenen Historie sein.

Schon Gutenberg betont, dass betrieblich relevante Größen nichtlinear voneinander abhängen. ${ }^{61}$ Die Komplexität der Strukturen in einem Unternehmen und zwischen Unternehmen und Umwelt verlangt geradezu eine nichtlineare Modellierung, soll sie der Forderung nach Realitätsnähe gerecht werden. ${ }^{62}$ In der betriebswirtschaftlichen Theorie werden nichtlineare Funktionen durchaus benutzt, allerdings in der Regel in statischen Modellen.

Die Produktionstheorie verwendet - wie auch das oben dargestellte Modell des Wirtschaftswachstums - neoklassische Produktionsfunktionen. Sie genügen dem Ertragsgesetz und sind homogen vom Grade $k \leq 1$, so dass die zugehörigen Kostenfunktionen sowohl bei partieller als auch bei totaler Faktorvariation (mit Ausnahme des linearhomogenen Falles) streng konvex verlaufen ${ }^{63}$ Die Gutenberg-Produktionsfunktion unterstellt bei intensitätsmäßiger Anpassung eine parabolische Abhängigkeit der Produktionskoeffizienten von der Intensität. Daraus ergibt sich ein s-förmiger Verlauf der Kostenfunktion. ${ }^{64}$

In der Absatztheorie werden einfach oder doppelt geknickte Preis-AbsatzFunktionen verwendet, um Marktunvollkommenheiten abzubilden. ${ }^{65}$ Die

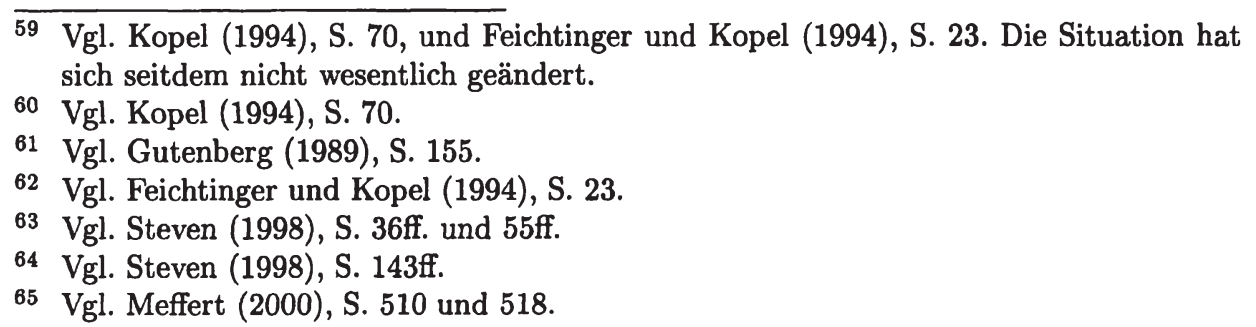


Knickstellen repräsentieren Schwellenwerte, an denen sich das Verhalten der Konkurrenz bzw. der Nachfrager sprunghaft ändert. Hyperbolische Nachfragefunktionen lassen sich aus Nutzenfunktionen mit konstanter Substitutionselastizität herleiten. Die Cobb-Douglas-Nutzenfunktion ist ein Spezialfall solcher sogenannter CES-Nutzenfunktionen. Zwischen Werbebudget und Nachfrage wird regelmäßig ein s-förmiger oder ertragsgesetzlicher Verlauf angenommen mit der Begründung, eine Intensivierung der Werbung führe zwar ceteris paribus zu einer Erhöhung der Nachfrage, ab einem gewissen $\mathrm{Maß}$ trete jedoch eine Sättigung des Marktes ein. ${ }^{66}$ Mit Sättigungseffekten und Schwellenwerten können Diffusionsprozesse neu eingeführter Produkte in nichtlinearer Form modelliert werden. ${ }^{67}$

Wirtschaftliches Handeln vollzieht sich im Zeitablauf. Statische Modelle, die zeitpunktbezogen in der Regel Gleichgewichtssituationen analysieren, können nur als erste Annäherung an die Realität verstanden werden. Im dynamischen Kontext sind die Modellvariablen häufig rückgekoppelt. Feedback tritt in hierarchischen Systemen wie der hierarchischen Produktionsplanung auf, wenn die Vorgaben der oberen Planungsebene von der unteren Ebene verarbeitet und die Ergebnisse rückgemeldet werden. ${ }^{68}$ Planungssysteme wie das Material Requirements Planning ${ }^{69}$, auf dem PPS-Systeme aufbauen, oder Bedienungssysteme ${ }^{70}$ basieren ebenfalls auf einer Abstimmung der einzelnen Ebenen durch Prioritäten und Rückkopplungen. Verhalten sich Entscheidungsträger aufgrund begrenzter Informationsverarbeitungskapazität myopisch, beispielsweise indem sie adaptive Erwartungen bilden, entsteht eine Rückkopplung dadurch, dass gegenwärtige Entscheidungen auf der Basis von Erfahrungen in der Vergangenheit getroffen werden. Des Weiteren sind Carry-Over-Effekte in Anpassungsprozessen, z.B. Werbung oder Investition, zu nennen, d.h. eine Werbeausgabe wirkt nicht nur in der aktuellen Periode, sondern auch - mit abnehmender Intensität - in späteren Perioden. Die erste betriebswirtschaftliche Anwendung der Chaostheorie behandelte chaotische Zeitpfade, die durch Investitionen ausgelöst wurden. ${ }^{71}$ Externe Effekte treten auf, wenn die Handlungen eines Individuums die Präferenzen oder verfügbaren Technologien anderer Individuen - seien es Haushalte oder Unternehmen - beeinflussen. Sie stellen in gewisser Hinsicht einen Spezialfall dar, da es sich hier um Rückkopplung über mehrere Individuen, nicht über die Zeit handelt.

\footnotetext{
66 Vgl. Busse von Colbe, Hammann und Laßmann (1992), S. 300.

67 Vgl. Granovetter und Soong (1983).

68 Vgl. Steven (1994), S. 27.

69 Vgl. Kistner und Steven (1993), S. 256.

70 In Erramilli und Forys (1991) werden Telefonschaltanlagen als Beispiel benutzt.

71 Vgl. Albach (1987).
} 
Die Aufzählung lässt sich beinahe beliebig erweitern, z.B. um asymmetrische Information, unterschiedliche Ablaufgeschwindigkeiten von Prozessen usw. ${ }^{72}$

Selbstverständlich ist chaotisches Verhalten bei nichtlinearer Rückkopplung nicht zwingend. Dies hat schon die Analyse der logistischen Parabel $x \mapsto a \cdot x(1-x)$ gezeigt, wo Chaos nur bei bestimmten Werten des Parameters $a$ auftritt.

Die - wie oben bereits erwähnt - erste Anwendung der Chaostheorie auf eine betriebswirtschaftliche Fragestellung stammt von Albach. Er stellt ein Modell des Unternehmenswachstums vor, in dem die treibende Kraft die Forschung und Entwicklung ist. Dem Management stehen zwei Parameter zur Verfügung: die Forschungsintensität als Anteil der Forschungsaufwendungen am befriedigenden Gewinn und die befriedigende Wachstumsrate als modellexogene, vom Management gewünschte Größe, mit denen es auf die Unternehmensentwicklung Einfluss nehmen kann. Der Zusammenbruch des Unternehmens tritt ein, wenn der Verschuldungsgrad negativ wird. Es zeigt sich, dass sowohl eine zu geringe Forschungsintensität durch monotone Schrumpfung als auch eine zu hohe Forschungsintensität durch chaotische Schwingungen den Zusammenbruch herbeiführen kann. ${ }^{73}$ Diese Arbeit hat eine Reihe von Weiterentwicklungen erfahren. Pinkwart variiert das Modell dahin gehend, dass er auf die befriedigende Wachstumsrate verzichtet und den Unternehmenszusammenbruch nicht an einer Überschuldung, sondern an einer Zahlungsunfähigkeit festmacht. ${ }^{74}$ Auch er kommt zu dem Ergebnis, dass sowohl zu hohe als auch zu niedrige Forschungsaufwendungen fatal sein können. Feichtinger und Kopel erweitern das Modell durch eine explizite Erfassung des tatsächlichen Entscheidungsverhaltens der Manager. In den vorherigen Versionen wurde das Management nur für die anfängliche Bestimmung der Parameter eingesetzt, danach blieb das Unternehmen gleichsam sich selbst überlassen. Die in der Mikroökonomik üblichen Annahmen der perfekten Voraussicht und vollständigen Rationalität werden nicht aufrecht erhalten, statt dessen findet ein Anchoring and Adjustment Verwendung. ${ }^{75}$

Im Supply Chain Management wird das Problem diskutiert, dass sich geringfügige Schwankungen der Endnachfrage über die Lieferkette hinweg zu immer größeren Schwankungen aufschaukeln. Dieses Phänomen ist als Bullwhip-Effekt bekannt ${ }^{76}$ und erinnert an den Schmetterlingseffekt. Wilding sowie Zäpfel und Wasner zeigen in zwei Aufsätzen auf, wie sich der

\footnotetext{
72 Vgl. Feichtinger und Kopel (1994), S. 23ff.

73 Vgl. Albach (1987), S. 14ff. und 55ff.

74 Vgl. Pinkwart (1992).

75 Vgl. Feichtinger und Kopel (1993) und Kopel (1994).

76 Vgl. Forrester (1972).
} 
Bullwhip-Effekt durch nichtlineare Rückkopplungen in der Lieferkette erklären lässt. ${ }^{77}$ Ähnlich von der Theorie des System Dynamics beeinflusst ist das Management-Modell von Rasmussen und Mosekilde. ${ }^{78}$

Die geometrische Seite der Chaostheorie, die fraktale Geometrie, nimmt Warnecke als Vorbild zur Entwicklung eines Organisationskonzepts. Vor dem Hintergrund eines turbulenten Umfelds empfiehlt er, Unternehmen in Organisationseinheiten zu unterteilen, die sich an den Eigenschaften von Fraktalen wie Selbstähnlichkeit und Dynamik orientieren. ${ }^{79}$

Einen ganzheitlichen Ansatz verfolgt Deser. Er betrachtet das Unternehmen als Gesamtsystem und nähert sich diesem auf drei Ebenen. Zum ersten stellt er die gegenwärtig aktuellen Forschungsmethoden im Hinblick auf nichtlineare dynamische Systeme vor; zum zweiten verwendet er den historisch gewachsenen, umgangssprachlichen Chaosbegriff unter der Überschrift „Phänomenologisches Chaos"; und zum dritten zeigt er die Erkenntnismöglichkeiten der modernen Chaosforschung auf, wobei er auf quantifizierende Aussagen jedoch vollständig verzichtet. ${ }^{80}$

Erwähnt werden soll schließlich noch eine Forschungsrichtung, die unter dem Oberbegriff „Chaossteuerung“" subsumiert werden kann. Es kann in der Realität zum Problem werden, die systembestimmenden Parameter so zu variieren, dass sie das System in stabile Regionen lenken. Dennoch ist es unter gewissen Voraussetzungen möglich, das System zu stabilisieren, indem man gezielt gegen die erratischen Schwankungen steuert. Die Methode ist unter dem Namen "Targeting“ bekannt. ${ }^{81}$ Dieser Fall spielt in den in der vorliegenden Arbeit vorzustellenden Modellen allerdings keine Rolle.

Als Beispiel für eine betriebswirtschaftliche Anwendung der Chaostheorie möge ein Warteschlangenmodell von Türschmann dienen. Das Modell ist sehr einfach strukturiert und daher instruktiv. ${ }^{82}$

Ausgangspunkt ist die allgemeine Bilanzgleichung

$$
\text { Endbestand = Anfangsbestand + Zugang - Abgang. }
$$

Das Geschehen vollzieht sich in diskreter Zeit, d.h. die Warteschlange verändert sich nur in bestimmten Zeitpunkten. Dann gilt für die Länge der

77 Vgl. Wilding (1998) sowie Zäpfel und Wasner (1999).

78 Vgl. Rasmussen und Mosekilde (1988) und auch den einführenden Artikel Andersen (1988).

79 Vgl. Warnecke $(1992,1993,1995)$.

80 Vgl. Deser (1997).

81 Vgl. Kopel $(1996,1997)$.

82 Vgl. Türschmann (1990). 


\section{KAPITEL 2. CHAOSTHEORIE IN DEN REALWISSENSCHAFTEN}

Warteschlange $L_{t+1}$ zum Zeitpunkt $t+1$

$$
L_{t+1}=L_{t}+Z_{t}-A_{t}
$$

mit dem Zugang $Z_{t}$ und dem Abgang $A_{t}$.

Es wird unterstellt, dass sich der Abgang proportional zur Länge der Warteschlange verhält

$$
A_{t}=L_{t} \cdot \mu,
$$

der Zugang erfolgt gemäß einer logistischen Gleichung:

$$
Z_{t}=L_{t} \cdot \gamma\left(1-\frac{L_{t}}{L^{\max }}\right)
$$

$\mu$ und $\gamma$ sind konstante positive Parameter, $L^{\max }$ ist die maximale Länge der Warteschlange. Bei $L^{\max }$ erfolgt kein Zugang mehr. Je geringer die Länge der Schlange ist, desto größer wird der Zugang bis zu einem Maximalwert für $Z_{t}$, der bei $L^{\max } / 2$ erreicht wird. Verkürzt man die Schlange noch weiter, wird auch der Zugang wieder geringer. Der erste Teil dieser Argumentation erscheint plausibel; Türschmann lässt hingegen offen, warum sich die Schlange für kleine Werte $Z_{t}$ auf die beschriebene Weise verhalten sollte.

Durch Einsetzen erhält man die Differenzengleichung

$$
L_{t+1}=L_{t}(1-\mu+\gamma)-\frac{\gamma}{L^{\max }} \cdot L_{t}^{2}
$$

die die Länge der Warteschlange unter Beachtung der Hypothesen über Zuund Abgang bestimmt. Die Variablensubstitution

$$
L_{t}=\frac{(1-\mu+\gamma) L^{\max }}{\gamma} \cdot x_{t}
$$

zeigt die topologische Konjugation ${ }^{83}$ zur logistischen Parabel. Man erhält nämlich

$$
x_{t+1}=(1-\mu+\gamma) x_{t}\left(1-x_{t}\right) .
$$

Das Verhalten der Warteschlange hängt vom Ausdruck $1-\mu+\gamma$ ab, durchläuft die in Abschnitt 3.23 ausführlich beschriebene Bifurkationskaskade und weist für gewisse Parameterwerte chaotisches Verhalten auf.

Die bisherigen Ausführungen haben gezeigt, dass nichtlineare rückgekoppelte Systeme zum einen grundsätzlich anfällig für Chaos sind und zum anderen durchaus Erkenntnisobjekte der Betriebswirtschaftslehre darstellen. Die

83 Vgl. Abschnitt 3.3.5. Topologisch konjugierte dynamische Systeme zeigen ein qualitativ übereinstimmendes Verhalten. 
so häufigen linearen oder statischen Modellierungen müssen als - bisweilen grobe - Vereinfachung angesehen werden. Warum sind dann Anwendungen der Chaostheorie auf betriebswirtschaftliche Fragestellungen nicht häufiger? Die Probleme sind zum einen auf der Ebene der Mathematik, zum anderen direkt in der Betriebswirtschaftslehre zu suchen.

Die mathematische Chaosforschung steckt auch nach über dreißig Jahren immer noch in den Anfängen. Allgemeine und leistungsfähige Theoreme stehen nach wie vor kaum zur Verfügung, und es ist fraglich, ob sie überhaupt existieren. Eine einheitlich anerkannte Definition von Chaos gibt es nicht; alle bisherigen Definitionsversuche sind problematisch. Jede Anwendung der Chaostheorie ruht also auf einem unsicheren mathematischen Fundament.

Die zur Beherrschung nichtlinearer dynamischer Systeme notwendige Mathematik ist hochkompliziert und wird von Ökonomen in der Regel nicht beherrscht. Die mathematische Handhabbarkeit bei der Modellierung ökonomischer Sachverhalte wird gewöhnlich durch Linearisierung sichergestellt. ${ }^{84}$ Hier kann sich der Ökonom auf ein mathematisches Theoriegebäude stützen, das gewissermaßen vollständig konstruiert ist. Die Bereitschaft, sich in die Theorie nichtlinearer Dynamik einzuarbeiten, wird hingegen schlecht belohnt. Man bewegt sich trotz aller Bemühungen auf recht dünnem Eis.

Chaostheoretische Modelle in der Ökonomik sind geprägt von einem hohen Abstraktionsgrad, um beherrschbar zu bleiben. Die verwendeten Systemgleichungen werden von nicht berücksichtigten Determinanten und stochastischen Einflüssen überlagert, und es ist gerade bei hochsensitiven Systemen keineswegs klar, wie sich die Einführung weiterer Variablen auswirkt. Dadurch bleibt der Anwendungsbereich der Modelle recht eingeschränkt; Verallgemeinerungen sind kaum möglich.

Darüber hinaus wird unterstellt, dass das System selbst autonom ist, die Systemgleichungen sich also über die Zeit hinweg nicht verändern. Man muss jedoch davon ausgehen, dass der Entscheidungsträger bei Auftreten chaotischer Zyklen in das System eingreift und es damit verändert. Die Analyse der Chaos verursachenden Einflussfaktoren in betriebswirtschaftlichen Modellen ist kein Selbstzweck, sondern notwendige Voraussetzung für die Entwicklung von Anpassungsmechanismen. Erst wenn man in der Lage ist, Chaos über-

84 Die Verwendung der Differentialrechnung stellt im Grunde genommen ebenfalls eine Linearisierung her, da die verwendeten Funktionen durch Tangenten approximiert werden. 
haupt als solches zu identifizieren, und weiß, unter welchen Voraussetzungen es auftreten kann, ist es möglich, geeignete Gegenmaßnahmen zu entwerfen ${ }^{85}$

Die Ziel der vorliegenden Arbeit besteht mithin darin, ein Bewusstsein für chaosgenerierende Mechanismen im Bereich der Koordination zu schaffen und mögliche Instrumente aufzuzeigen, die helfen können, mit den unerwünschten Auswirkungen des Chaos fertig zu werden. Die notwendig strengen Voraussetzungen der Modelle verbieten allerdings eine direkte Umsetzung in praktische Handlungsanweisungen. Insofern handelt es sich um deskriptive, nicht normative Theorie.

85 Genauso muss man in der Kostenrechnung erst eine Analyse der Soll-Ist-Abweichungen der angefallenen Kosten durchführen, bevor geeignete Korrekturen durchgeführt werden können. 


\section{Kapitel 3}

\section{Nichtlineare Dynamik}

In diesem Kapitel wird das methodische Fundament gelegt, indem die mathematischen Werkzeuge bereitgestellt werden, die zur Erklärung komplexen, chaotischen Verhaltens in dynamischen Systemen notwendig sind. Wir werden sehen, dass bereits einfache, nichtlineare Rückkopplungssysteme ein sehr komplex erscheinendes Verhalten an den Tag legen können, obwohl die zugrunde liegenden Systemgleichungen frei von stochastischen Einflüssen sind. Dafür steht der Begriff deterministisches Chaos.

Nach einer kurzen Einordnung der Nichtlinearen Dynamik in die Systemtheorie werden die grundlegenden Definitionen der Theorie der dynamischen Systeme vorgestellt, mit denen im Weiteren zu arbeiten ist. Zum Begriff des deterministischen Chaos existiert bislang keine eindeutige Definition. Die exakteste und anschaulichste Begriffsfassung, das topologische Chaos nach Devaney, wird als Erstes vorgestellt und auf einfache Funktionen angewendet. Das Konzept der topologischen Konjugation wird die Verbindung dieser Systeme untereinander und zu einer großen Zahl weiterer Systeme herstellen. Die Konsequenzen der topologischen Konjugation sind weit reichend: Die Diskussion der einfachen Systeme erscheint sehr speziell und für eine sinnvolle Anwendung auf reale Rückkopplungssysteme unbrauchbar. Die topologische Konjugation wird zeigen, dass alle Ergebnisse auf viele Systeme übertragbar sind, die nur wesentlich allgemeineren Bedingungen genügen müssen.

Nichtlineare Systeme bereiten einer strengen Analyse erhebliche, teils sogar unlösbare Probleme. Oftmals ist man gezwungen, sich auf eine Untersuchung in numerischen Simulationsrechnungen zu beschränken. Zunächst wird diskutiert, welchen Beitrag Simulationen vor dem Hintergrund der dargestellten Eigenschaften chaotischer Systeme überhaupt leisten können. Danach werden verschiedene numerische Analysemethoden vorgestellt und eine 
darauf aufbauende Definition für numerisches Chaos formuliert. Eine Beweiskraft in mathematischem Sinne besitzen jedoch auch noch so ausgefeilte numerische Methoden selbstverständlich nicht.

Die Darstellung von Chaos in mehrdimensionalen Systemen erfordert einen eigenen Begriffsapparat; insbesondere der Begriff des Seltsamen Attraktors spielt eine wichtige Rolle. Ein Exkurs resümiert Eigenschaften linearer Systeme, auf die die Analyse nichtlinearer Systeme zurückgreift; ein weiterer Exkurs zeigt die Sonderrolle zeitstetiger Systeme der Dimension eins und zwei auf. Die topologische Definition nach Devaney lässt sich zwar prinzipiell auf mehrdimensionale Systeme übertragen, und es gibt auch weitere Definitionen, auf die kurz eingegangen wird. Doch wegen der großen technischen Schwierigkeiten im Umgang mit mehrdimensionalen Systemen haben numerische Methoden eine große Bedeutung. Die für eindimensionale Systeme dargestellten Konzepte werden daher auf den mehrdimensionalen Fall erweitert. Mit dem Hénon-Attraktor und dem Rössler-Attraktor wird je ein Beispiel für ein System in zeitdiskreter und in zeitstetiger Zeit vorgestellt.

Die Bifurkationstheorie schließlich wird genauer aufzeigen, welche Strukturen in den besprochenen Systemen verborgen sind. Eine Bifurkation ist eine Änderung des qualitativen Systemverhaltens, die von der Änderung eines Parameterwertes verursacht wird. Dem Parameter kommt mithin eine systembestimmende Bedeutung zu; die Kontrolle solcher Parameter eröffnet die Möglichkeit, das Systemverhalten zu steuern.

\subsection{Systemtheorie und Kybernetik}

Die allgemeine Systemtheorie definiert ein System als eine Menge von Elementen, die miteinander in Beziehung stehen ${ }^{86}$ Die Elemente, die Systemvariablen heißen, werden in der Menge $A=\left\{a_{1}, \ldots, a_{n}\right\}$ zusammengefasst, das Element $a_{0}$ ist die Umwelt des Systems. Die Beziehungen zwischen den Elementen bzw. zwischen Elementen und Umwelt werden mit $r_{i j}, \quad i, j=0, \ldots, n$ bezeichnet und bilden die Menge $R$.

\section{Definition 3.1}

Ein System ist eine Menge $S=\{A, R\}$. A heißt Universum, $R$ die Charakteristik von $S$.

Die Elemente des Systems sind Entitäten, deren innere Struktur außerhalb der Betrachtung bleibt; sie werden nur durch ihre Relationen beschrieben.

${ }_{86}$ Vgl. Krallmann (1996), S. 6. 
Ein Element $a_{i}$ erhält von anderen Elementen $a_{j}$ Input und reagiert darauf, indem es an andere Elemente Output abgibt, den diese wiederum als Input verwenden. Die Reaktion des Elements wird durch eine Transformationsregel $\mathrm{T}_{i}$ erfasst:

$$
\mathbf{w}_{i}=\mathbf{T}_{i}\left(\mathbf{v}_{i}\right)
$$

mit $\mathbf{v}_{i}$ als Input und $\mathbf{w}_{i}$ als Output des Elements. Die Vektordarstellung impliziert, dass sich Input und Output aufspalten lassen. Die Kopplungen der Elemente können durch Vektoren $\mathbf{v}_{i j}$ angegeben werden, die die gemeinsamen Elemente der Vektoren $\mathbf{w}_{i}$ und $\mathbf{v}_{j}$ enthalten, also angeben, welche Outputs des Elements $a_{i}$ von Element $a_{j}$ als Input verwendet werden. ${ }^{87}$ Man unterscheidet die drei folgenden Typen von Kopplungen. ${ }^{88}$

Serielle Kopplung liegt vor, wenn der Fluss nur in einer Richtung von $a_{i}$ nach $a_{j}$ verläuft, der Vektor $\mathbf{v}_{j i}$ also nicht existiert. Je nach Gestalt von $\mathbf{v}_{i j}$ kann man vier Unterfälle trennen:

a) lose Kopplung: $\mathbf{v}_{i j} \neq \mathbf{w}_{i}, \mathbf{v}_{i j} \neq \mathbf{v}_{j}$

b) lose Input-Kopplung: $\mathbf{v}_{i j}=\mathbf{w}_{i}, \mathbf{v}_{i j} \neq \mathbf{v}_{j}$

c) lose Output-Kopplung: $\mathbf{v}_{i j} \neq \mathbf{w}_{i}, \mathbf{v}_{i j}=\mathbf{v}_{j}$

d) enge Kopplung: $\mathbf{v}_{i j}=\mathbf{w}_{i}=\mathbf{v}_{j}$

Parallele Kopplung liegt vor, wenn $a_{i}$ und $a_{j}$ Input von einem dritten Element $a_{k}$ erhalten. Man unterscheidet hier zwei Unterfälle:

a) balancierte Kopplung: $\mathbf{v}_{k i}=\mathbf{v}_{k j}$

b) nicht-balancierte Kopplung: $\mathbf{v}_{\boldsymbol{k} i} \neq \mathbf{v}_{\boldsymbol{k} j}$

Rückkopplung schließlich bedeutet, dass der Input eines Elements $a_{i}$ teilweise zum Input desselben Elements wird, formal dass der Vektor $\mathbf{v}_{i i}$ existiert. Rückkopplungen spielen bei der Analyse chaotischer Modelle eine große Rolle.

Die Umwelt stellt ein Element außerhalb des Systems dar. Das Verhalten eines Systems wird dargestellt als Input-Output-Relation zwischen System und Umwelt. Die Umwelt übt einen Stimulus auf das System aus, bezeichnet als $\mathbf{x}$, worauf das System mit dem Respons y reagiert:

$$
\mathbf{y}=\mathbf{T}(\mathbf{x}) \text {. }
$$

Die Elemente der Vektoren $\mathbf{x}$ und $\mathbf{y}$ werden als partielle Stimuli und Response bezeichnet. Da es für eine Input-Output-Relation keinen formalen

87 Die Kopplungen $v_{i j}$ dürfen nicht mit den Relationen $r_{i j}$ identifiziert werden. Die $v_{i j}$ geben nur den direkten Fluss von $a_{i}$ nach $a_{j}$ an, die $r_{i j}$ sind Symbole für die Beziehung zwischen $a_{i}$ und $a_{j}$, die auch indirekt über intermediäre Elemente bestehen kann. Insofern stehen die $r_{i j}$ auf einem abstrakteren Level.

Vgl. Klír und Valach (1967). 
Unterschied macht, ob sie innerhalb des Modells oder über die Systemgrenzen hinweg besteht, könnte man statt $\mathbf{y}$ und $\mathbf{x}$ auch $\mathbf{w}_{0}$ und $\mathbf{v}_{0}$ schreiben.

Ist bei der Transformation von Input in Output der Zusammenhang eindeutig, liegt eine Funktion $\mathbf{y}=\mathbf{f}(\mathbf{x})$ vor. Das System reagiert auf denselben Stimulus immer gleich.

Bei mehrwertiger Transformation sind zwei Fälle zu unterscheiden. Erstens liegt eine mehrwertige Stimulus-Respons-Beziehung vor, wenn das System stochastischen Einflüssen ausgesetzt ist. Dann hängt der Respons auch von einer Zufallsvariablen ab, die den Stimulus überlagert. Zweitens kann in einem dynamischen System ein Respons von einer Sequenz von Stimuli abhängen. Unterschiedliche Response auf ein und denselben Stimulus werden dadurch verursacht, dass der Stimulus verschiedenen Sequenzen angehört. In diesem Fall muss das System die vorherigen Stimuli erinnern können. Dies wird abgebildet im Status des Systems $\mathbf{s}_{t}$, der abhängt von Status und Input des Systems zu einem früheren Zeitpunkt. Der Respons lässt sich damit als Funktion des gegenwärtigen und aller vergangenen Stimuli formulieren:

$$
\mathbf{y}_{t}=\mathbf{f}\left(\mathbf{x}_{t}, \mathbf{s}_{t}\left(\phi\left(\mathbf{x}_{t-1}, \mathbf{s}_{t-1}\right)\right)\right.
$$

Die Abgrenzung von System und Umwelt wie auch die Abgrenzung der Elemente untereinander, der Auflösungsgrad, hängt vom Analysezweck ab. Es besteht ein Unterschied zwischen dem System selbst und der Beobachtung bzw. Beschreibung eines Systems. Letztere ist eine - bewusst oder unbewusst - immer theoriegeleitete Abbildung der Realität, die von allen nicht interessierenden Aspekten abstrahiert, sie ist ein Modell eines real oder in der Vorstellung existierenden Systems.

Die Klassifikation von Systemen erfolgt nach verschiedenen Kriterien. Man kann Systeme in natürliche und künstliche einteilen nach der Beteiligung des Menschen an der Entstehung. Eine andere Einteilung ist die in ideelle, also nur in der Vorstellung existierende, und reale Systeme. Ideelle Systeme sind immer künstlich, alle Modelle fallen in diese Kategorie.

Weiterhin kann man Systeme in offene und geschlossene einteilen, wobei letztere nicht mit ihrer Umwelt in Beziehung stehen. Dynamische Systeme verändern sich im Zeitablauf, statische Systeme bleiben unverändert. Nach der Art der Relationen kann man schließlich lineare und nichtlineare Systeme unterteilen. Die drei letztgenannten Merkmale betreffen genau genommen Modelle. Real existierende Systeme sind immer offen, dynamisch und nichtlinear. Die Beziehungen zur Umwelt, der Veränderungsdruck oder die 
Nichtlinearität können aber so schwach ausgeprägt sein, dass sie in der Modellierung vernachlässigt werden können. Die Klassifikation von Systemen ist in Abbildung 3.1 schematisch dargestellt.

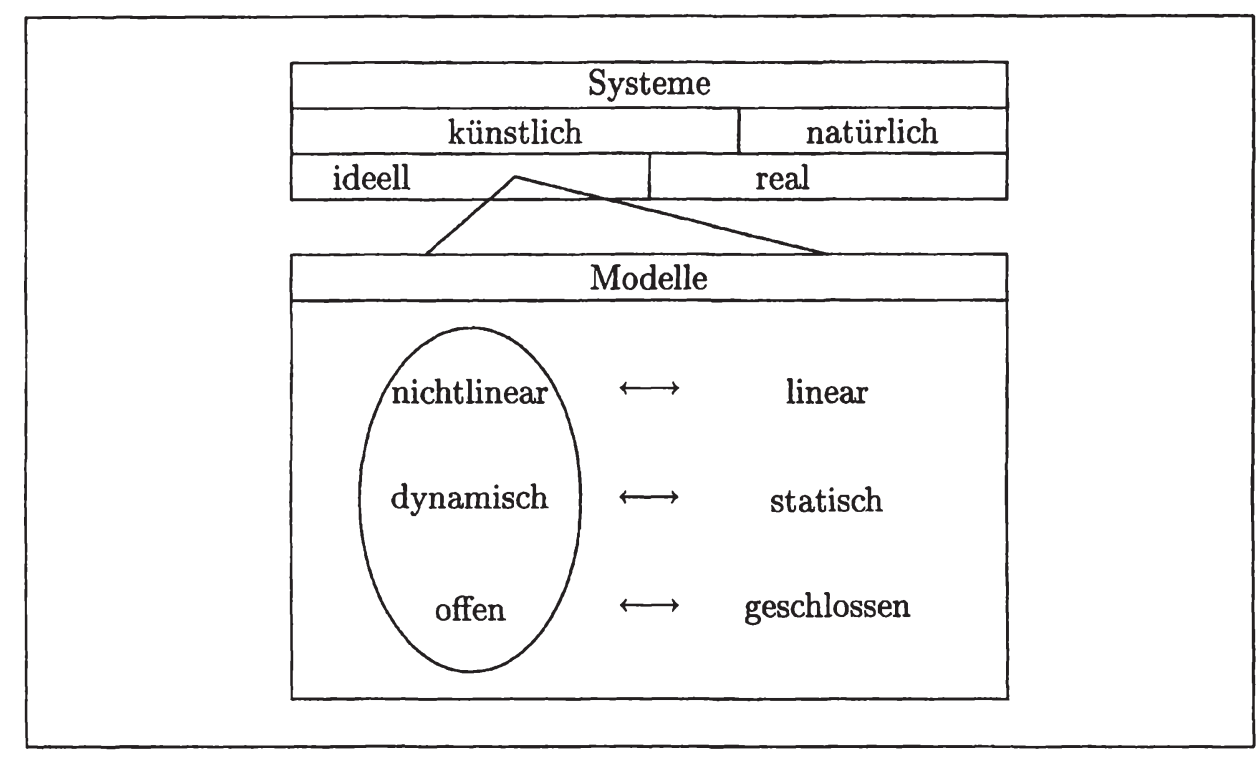

Abbildung 3.1: Klassifikation von Systemen

Ein Sonderfall ist die Einteilung in stochastische und deterministische Systeme. Reale Systeme sind streng genommen stochastisch wegen der Heisenbergschen Unschärferelation. Auf makroskopischer Ebene spielt diese allerdings keine Rolle, so dass man von deterministischen Systemen sprechen kann. Oft sind jedoch nicht alle nötigen Informationen über Struktur und Verhalten des Systems bekannt. Unbekannte Einflussfaktoren werden dann als stochastische Einflüsse interpretiert und mit Wahrscheinlichkeitsverteilungen modelliert.

\subsection{Grundlegende Begriffe dynamischer Systeme}

Die Modellierung der Zeit in dynamischen Systemen kann auf zwei Weisen erfolgen, deren Wahl für die Modellergebnisse weitreichende Folgen hat. In zeitstetigen Systemen ist die Zeit ein Kontinuum, die Systemvariablen können sich beständig ändern. Zeitdiskrete Systeme werden hingegen als Folge von Zeitpunkten interpretiert, zwischen denen die Systemvariablen konstant 
bleiben. Wie später gezeigt wird, können stetige Systeme erst ab drei Systemvariablen chaotisches Verhalten zeigen, während in diskreten Systemen bereits eine Variable genügt. ${ }^{89}$

Ein stetiges System wird beschrieben durch ein System von Differentialgleichungen:

$$
\dot{\mathbf{x}}=\mathbf{f}(\mathbf{x}(t))
$$

mit dem Systemzustand $\mathbf{x} \in X$, dem Phasenraum $X \subset \mathbb{R}^{n}$ und der kontinuierlich gemessenen Zeit $t \in \mathbb{R}$. $\mathbf{f}$ ist eine $n$-dimensionale vektorwertige Funktion, die Systemzustand und Änderungsgeschwindigkeit zu einem Zeitpunkt miteinander verknüpft.

Ein diskretes System wird dargestellt durch ein System von Differenzengleichungen ${ }^{90}$ :

$$
\mathbf{x}_{t+1}=\mathbf{f}\left(\mathbf{x}_{t}\right)
$$

Im Unterschied zu oben gilt hier $t \in \mathbb{Z}$ und $\mathbf{f}$ verknüpft zwei Systemzustände $\mathrm{zu}$ aufeinander folgenden Zeitpunkten miteinander.

$\mathrm{Zu}$ beiden Systemen gehört ein Fluss

$$
\mathbf{x}_{t}=\Phi_{t}\left(\mathbf{x}_{0}\right) \quad \text { bzw. } \quad \mathbf{x}(t)=\Phi\left(t, \mathbf{x}_{0}\right)
$$

der den Systemzustand $\mathbf{x}_{t}$ bzw. $\mathbf{x}(t)$ zum Zeitpunkt $t$ bei gegebenem Anfangswert $\mathbf{x}_{0}$ angibt. Eine Folge von $x_{t}$ in einem diskreten System nennen wir Bahn, ein Intervall von $x(t)$ heißt Trajektorie. Mit Zeitreihe sind empirische Daten gemeint.

Das Augenmerk liegt zunächst auf zeitdiskreten, eindimensionalen Systemen. Der Grund liegt darin, dass solche Systeme vergleichsweise einfach strukturiert sind. Dennoch lassen sich hieran alle wesentlichen Eigenschaften des Chaos, wie sie später definiert werden, erläutern.

Das grundlegende Prinzip eines zeitdiskreten Systems der Form (3.2) ist die Rückkopplung. Gleichung (3.2) bildet einen Regelkreis wie in Abbildung 3.2 ab. Die Folge der $x_{t}$ entsteht durch Iteration, d.h. durch wiederholtes Anwenden der Funktion $f$ auf den Startwert $x_{0}$, auf $f\left(x_{0}\right)$, auf $f\left(f\left(x_{0}\right)\right)$ usw. Auf diese Weise ist die Kenntnis des Flusses $\Phi$ nicht notwendig. Wir definieren daher:

89 Die Begriffe ,stetig" und „diskret" beziehen sich als Eigenschaft eines Systems immer auf den Zeitverlauf. Insbesondere der Begriff ,stetig“ darf nicht als Eigenschaft einer Funktion missverstanden werden.

90 Die zu Gleichung (3.1) analoge Darstellung mit Hilfe des Differenzenoperators $\Delta \mathbf{x}_{t+1}=$ $\mathbf{f}\left(\mathbf{x}_{t}\right)-\mathbf{x}_{\mathrm{t}}$ wird aus praktischen Gründen nicht verwendet. 


\section{Definition 3.2}

Sei $f: X \rightarrow X$ eine Abbildung. Dann ist die Abbildung $f^{t}: X \rightarrow X$ rekursiv definiert durch $f^{0}(x)=x$ und $f^{t}(x)=f \circ f^{t-1}(x)$ mit $t \in \mathbb{N}$ und $x \in X$. Sei nun $f$ außerdem bijektiv. Dann ist $f^{-t}(x)=f^{t-1} \circ f^{-1}(x)$.

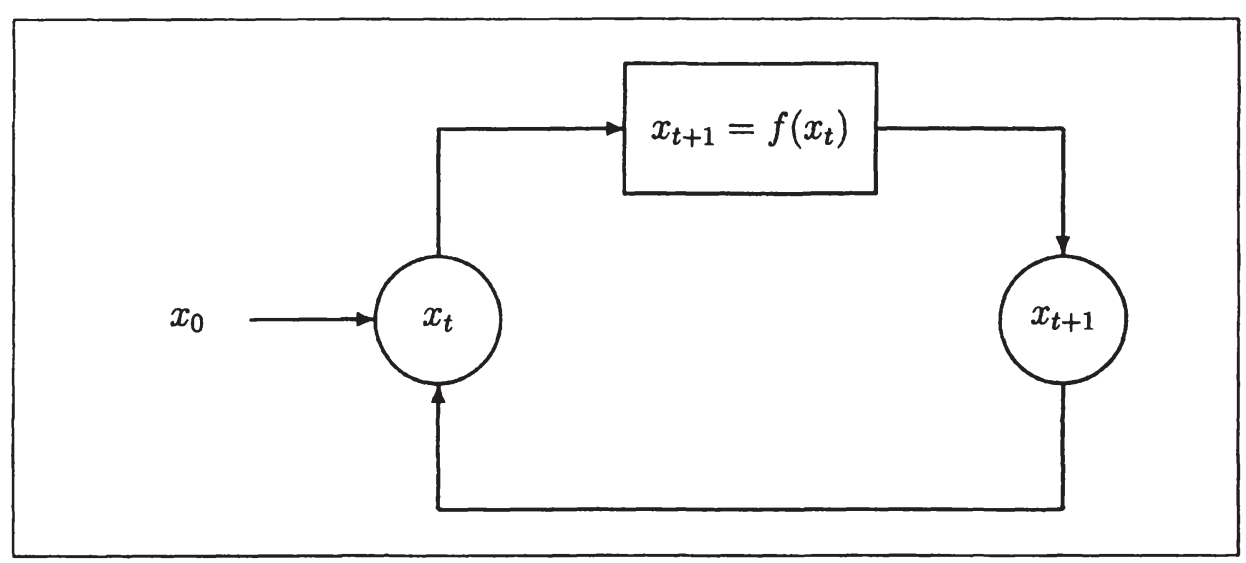

Abbildung 3.2: Grundform des kybernetischen Regelkreises

Wir stellen nun in den folgenden Definitionen einige Begriffe zusammen, mit denen sich das Verhalten eines dynamischen Systems beschreiben lässt.

\section{Definition 3.3}

Die Menge $\left\{f^{t}(x): t \in \mathbb{Z}\right\}$ für ein gewisses $x \in X$ unter einer bijektiven Abbildung $f: X \rightarrow X$ heißt Orbit von $x$. Ist $f$ nicht bijektiv, heißt die Menge $\left\{f^{t}(x): t \in \mathbb{N}\right\}$ Vorwärtsorbit von $x .^{91}$

\section{Definition 3.4}

Ein Punkt $x$ heißt periodischer Punkt der Periode $t$, wenn gilt: $f^{t}(x)=x$. Im Spezialfall $t=1$ heißt $x$ Fixpunkt von $f .{ }^{92}$

Ein Fixpunkt eines dynamischen Systems wird auch als Gleichgewicht bezeichnet. ${ }^{93}$ Ein geschlossenes System verlässt einen einmal erreichten Fixpunkt nicht mehr, es verharrt in diesem Zustand.

Der Orbit eines periodischen Punktes enthält genau $t$ Elemente, die sich ständig wiederholen. Auch dies kann man als Gleichgewicht bezeichnen, da der Zustand des Systems alle $t$ Perioden derselbe ist. Damit sind periodische

\footnotetext{
91 Vgl. Devaney (1989), S. 17.

92 Vgl. Devaney (1989), S. 18.

93 Vgl. Kopel (1994), S. 36.
} 
Punkte genau das Gegenteil dessen, was man unter Chaos versteht. Oder anders ausgedrückt: Eine Abbildung, deren Definitionsbereich ausschließlich periodische Punkte enthält, wird man zumindest dann nicht als chaotisch bezeichnen, wenn die Periode so klein ist, dass das Muster in der Zeitreihe erkennbar ist.

Von entscheidender Bedeutung ist die Frage, ob ein dynamisches System sich auf einen Fixpunkt oder periodischen Zyklus einpendelt, mit anderen Worten, ob es stabil ist. Dazu führen wir den Begriff des Attraktors ein, der den Endzustand eines stabilen Systems beschreibt. ${ }^{94}$

\section{Definition 3.5}

Ein Attraktor eines dynamischen Systems ist eine kompakte Menge $A$, die invariant unter dem Fluss $\Phi$ ist und zu der eine Umgebung $U$ existiert, aus der $A$ Punkte anzieht: $\Phi_{t}(A)=A$ für alle $t$ und $\lim _{t \rightarrow \infty} \Phi_{t}(U)=A$. Gilt für eine kompakte, invariante Menge $\lim _{t \rightarrow-\infty} \Phi_{t}(U)=A$, wird sie Repellor genannt.

\section{Definition 3.6}

Sei $p$ ein periodischer Punkt der Primperiode ${ }^{95} t$. Der Punkt $p$ heißt hyperbolisch, wenn $\left|\left(f^{t}\right)^{\prime}(p)\right| \neq 1 .^{96}$

Hyperbolische Punkte lassen Rückschlüsse auf das Verhalten eines dynamischen Systems zu, wie der folgende Satz zeigt: ${ }^{97}$

\section{Satz 3.1}

Sei $f: X \rightarrow X$ stetig differenzierbar und $p$ ein hyperbolischer Fixpunkt mit $\left|f^{\prime}(p)\right|<1$. Dann ist $p$ ein Attraktor.

Beweis. Die Invarianz ist nach Voraussetzung klar. Es ist zu zeigen, dass es eine offene Umgebung $U$ um $p$ gibt, so dass ${ }^{98}$

$$
\lim _{t \rightarrow \infty} f^{t}(x)=p \text { für alle } x \in U .
$$

Da $f$ stetig differenzierbar ist, existiert ein $\epsilon>0$ mit $\left|f^{\prime}(x)\right|<c<1$ für $x \in[p-\epsilon, p+\epsilon]$. Aus dem Mittelwertsatz der Differentialrechnung ${ }^{99}$ ergibt

94 Die Definition lässt sich auf stetige und diskrete Systeme jeder Dimension anwenden.

95 Ein Punkt der Periode $t$ ist auch periodisch mit Periode $k \cdot t, k \in \mathbb{N}$. Mit Primperiode wird die kleinste Periode bezeichnet.

96 Vgl. Devaney (1989), S. 24.

97 Vgl. Devaney (1989), S. 24.

98 Man beachte, dass in Differenzengleichungen gilt: $\Phi_{t}(x)=f^{t}(x)$.

99 Vgl. Heuser (1994), S. 279. 
sich:

$$
|f(x)-p|=|f(x)-f(p)| \leq c|x-p|<|x-p| \leq \epsilon .
$$

Damit hat man $f(x) \in[p-\epsilon, p+\epsilon]$ und der Abstand von $f(x)$ zu $p$ ist in der Tat kleiner als der von $x$ zu $p$. Wiederholt man diese Argumentation $n$-mal, erhält man

$$
\left|f^{t}(x)-p\right| \leq c^{t}|x-p|
$$

Wegen $\lim _{t \rightarrow \infty} c^{t}=0$ hat man auch $\lim _{t \rightarrow \infty}\left|f^{t}(x)-p\right|=0$, so dass $f^{t}(x) \rightarrow p$ für $t \rightarrow \infty$.

\section{Corollar 3.2}

Sei $f: X \rightarrow X$ stetig differenzierbar und $p$ ein hyperbolischer periodischer Punkt der Periode $n$ mit $\left|\left(f^{n}\right)^{\prime}(p)\right|<1$. Dann gibt es eine offene Umgebung $U$ um $p$, so dass

$$
\lim _{t \rightarrow \infty} f^{t \cdot n}(x)=p \text { für alle } x \in U .
$$

Beweis. Man zeigt dies, indem man im Beweis zu Satz 3.1 jeweils $f$ durch $f^{n}$ ersetzt.

Auch im Zusammenhang mit dem qualitativen Verhalten eines dynamischen Systems bei Änderung eines exogenen Parameters spielen hyperbolische Punkte eine Rolle. In Abschnitt 3.6 wird gezeigt, dass man eine qualitative Veränderung des Systemverhaltens nur in der Nähe nicht-hyperbolischer Punkte erwarten kann.

\subsection{Topologisches Chaos}

\subsubsection{Die Definition nach Devaney}

Für den Begriff des Chaos gibt es keine einheitliche, allgemein anerkannte Definition. Hier wird zunächst die Definition von Devaney zugrunde gelegt. Sie basiert auf topologischen Eigenschaften und eignet sich für zeitdiskrete, ein- und mehrdimensionale Systeme: ${ }^{100}$

\section{Definition 3.7}

Eine Abbildung $f: X \rightarrow X$ heißt chaotisch auf $X$, wenn

1. $f$ sensitive Abhängigkeit von den Anfangsbedingungen aufweist, 100 Vgl. Devaney (1989), S. 50. 
2. $f$ topologisch transitiv ist und

3. periodische Punkte dicht in $X$ liegen.

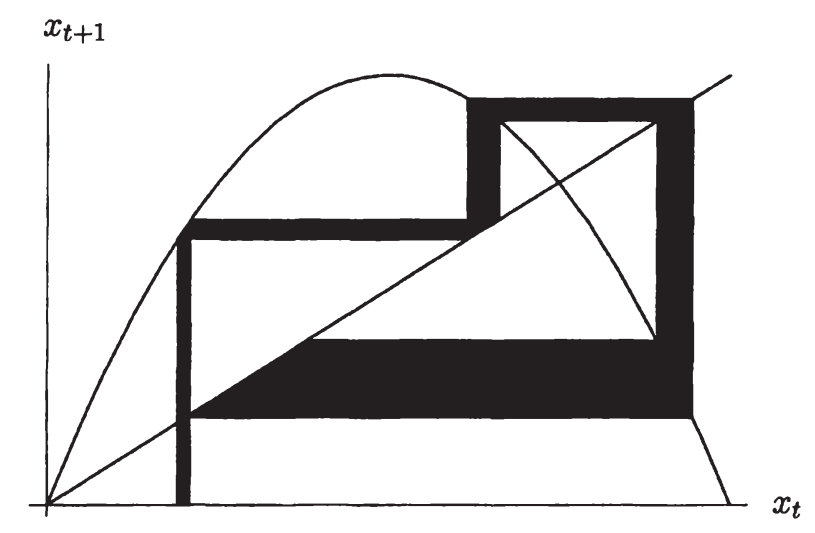

In Anlehnung an: Peitgen, Jürgens und Saupe (1994), S. 41

Abbildung 3.3: Sensitive Abhängigkeit von den Anfangsbedingungen

Diese Eigenschaften bedürfen der Erläuterung. In den Graphen wird die Parabel $x \mapsto 4 x(1-x)$ verwendet, deren Chaoseigenschaft später nachgewiesen wird.

Sensitive Abhängigkeit von den Anfangsbedingungen kann man anschaulich so erklären: $\mathrm{Zu}$ jedem Anfangswert $x_{0} \in X$ gibt es beliebig nahe Werte, deren Bahnen nach einer gewissen Anzahl von Iterationen völlig anders aussehen, als die Bahn von $x_{0}$. Dies ist die bedeutsamste und auffälligste Eigenschaft chaotischer Systeme und ist der Grund dafür, dass sich chaotische Systeme einer langfristigen Prognose widersetzen. Ein Messfehler, und mag er noch so winzig sein, wird dazu führen, dass die prognostizierte und die tatsächliche Bahn auch ohne jeden stochastischen Einfluss keine Ähnlichkeit miteinander haben. Dazu vergleiche man Abbildung 3.3. Wir wollen diese Eigenschaft in eine exakte Definition fassen: ${ }^{101}$

\section{Definition 3.8}

$f: X \rightarrow X$ weist sensitive Abhängigkeit von den Anfangsbedingungen auf, wenn es ein $\delta>0$ gibt, so dass es für jedes $x \in X$ und jede Umgebung $U$ von $x$ ein $y \in U$ und ein $t \geq 0$ gibt mit

$$
\left|f^{t}(x)-f^{t}(y)\right|>\delta
$$

101 Vgl. Devaney (1989), S. 49. 
$\mathrm{Zu}$ beachten ist bei dieser Definition, dass das einmalige Überschreiten des Wertes $\delta$, auch Sensitivitätskonstante genannt, genügt. Für das Verhalten der Bahnen an anderen Stellen $t$ wird nichts gefordert.

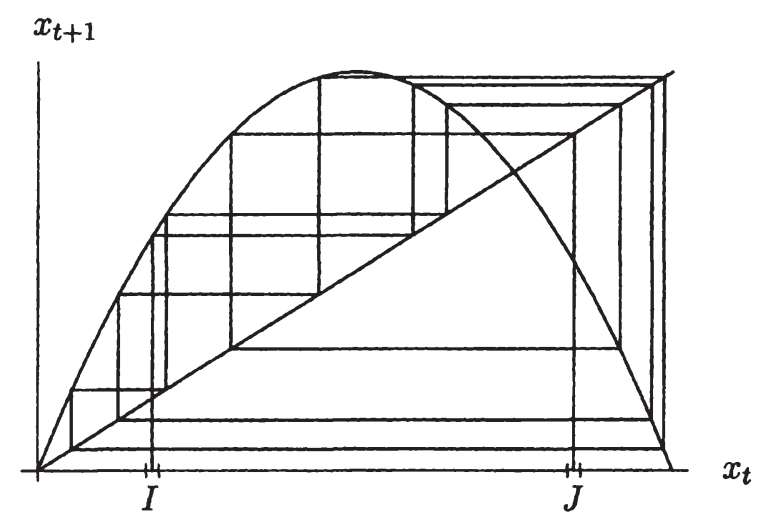

In Anlehnung an: Peitgen, Jürgens und Saupe (1994), S. 52

Abbildung 3.4: Topologische Transitivität

Topologische Transitivität bedeutet, dass es in jedem beliebig kleinen Intervall Punkte gibt, die durch Iteration schließlich jedes andere beliebig kleine Intervall erreichen. ${ }^{102}$ In Abbildung 3.4 genügen zehn Iterationen, damit der Startwert von Intervall $I$ nach $J$ gelangt. Dadurch kann das dynamische System nicht in endlich viele disjunkte offene Teilmengen von $X$ zerlegt werden, die unter Iteration invariant bleiben. ${ }^{103}$

\section{Definition 3.9}

$f: X \rightarrow X$ heißt topologisch transitiv, wenn es für jedes Paar offener Mengen $U, V \subset X$ ein $t>0$ gibt, so dass

$$
f^{t}(U) \cap V \neq \emptyset \text {. }
$$

Der Begriff des periodischen Punktes ist bereits in Definition 3.4 erklärt worden. In einem chaotischen System sollen solche Punkte nun dicht liegen.

\section{Definition 3.10}

Periodische Punkte liegen dicht, wenn in jeder Umgebung $U$ eines Punktes $x_{0} \in X$ mindestens ein periodischer Punkt gefunden werden kann.

102 Vgl. Devaney (1989), S. 49.

${ }^{103}$ Eine Teilmenge von $X$ bleibt invariant, wenn die Bahnen aller Anfangswerte aus dieser Teilmenge in ihr verbleiben. 


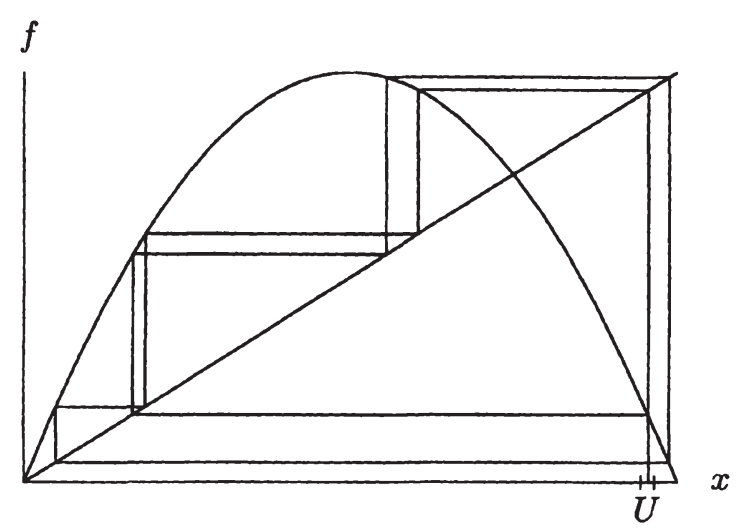

Abbildung 3.5: Dichte periodische Punkte

Abbildung 3.5 zeigt exemplarisch einen periodischen Zyklus, der in einer willkürlich gewählten Umgebung $U$ beginnt. Wie auch immer man $U$ wählt, es findet sich ein periodischer Punkt in dieser Umgebung.

Ein chaotisches System im Sinne obiger Definition enthält also drei Elemente: Unvorhersagbarkeit wegen der sensitiven Abhängigkeit, Unzerlegbarkeit wegen der topologischen Transitivität und dazu ein Element der Regularität wegen der dichten periodischen Punkte. ${ }^{104}$

\subsubsection{Chaos in der Sägezahnfunktion}

Wir beginnen damit, die sogenannte Sägezahnfunktion zu analysieren, für die sich die Chaoseigenschaften sehr leicht nachweisen lassen. ${ }^{105}$ Ersetzungsregeln wie die bereits erwähnte topologische Konjugation werden dann zeigen, dass sich die Ergebnisse verallgemeinern lassen. Auf diese Weise werden wir im folgenden Abschnitt Chaos für die Zeltfunktion nachweisen und von dort im übernächsten Abschnitt auf die logistische Parabel übertragen.

Die Sägezahnfunktion ist folgendermaßen definiert:

$$
S:[0,1] \rightarrow[0,1], \quad S(x)= \begin{cases}2 x & \text { für } x<0,5 \\ 2 x-1 & \text { für } x \geq 0,5\end{cases}
$$

104 Vgl. Loistl und Betz (1996), S. 42.

105 Die Darstellung, insbesondere die Beweise, folgen Peitgen, Jürgens und Saupe (1994), S. $88 \mathrm{ff}$. 
Der Graph ist in Abbildung 3.6 dargestellt.

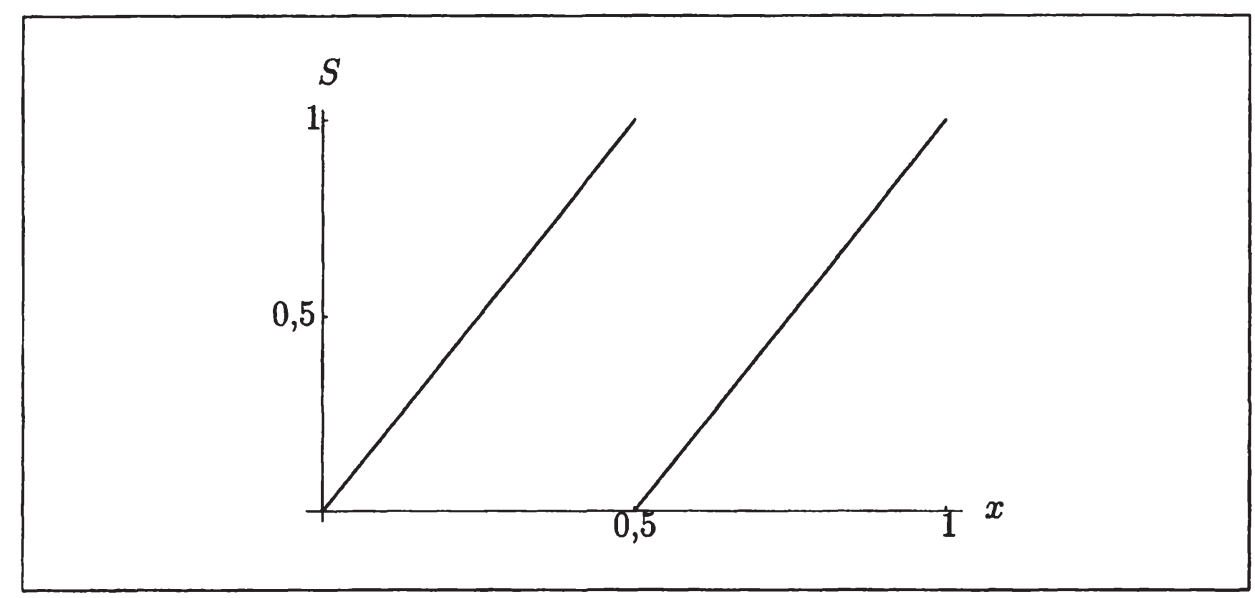

Abbildung 3.6: Graph der Sägezahnfunktion

Für diese Funktion lässt sich Chaos am einfachsten nachweisen, wenn man zu binären Zahlen übergeht. Jede Zahl aus dem Einheitsintervall wird als Folge binärer Ziffern dargestellt, und die Sägezahnfunktion verschiebt dann die Ziffern um eine Stelle nach links, wobei die Stelle vor dem Komma abgeschnitten wird ${ }^{106}$ :

$$
x=0 . a_{1} a_{2} a_{3} \cdots \rightarrow S(x)=0 . a_{2} a_{3} a_{4} \cdots
$$

\section{Satz 3.3}

$S$ weist sensitive Abhängigkeit von der Anfangsbedingung auf.

Beweis. Wir wählen $\delta \in(0,1 / 2)$ und ein $y_{0}$, das in den ersten $t$ binären Stellen mit $x_{0}$ übereinstimmt. Dann ist $\left|x_{0}-y_{0}\right| \leq 2^{-t}$. Nach $t$ Iterationen sind die übereinstimmenden Stellen eliminiert, es ist $\left|x_{t}-y_{t}\right|=1 / 2$.

Die Eigenschaft der Transitivität lässt sich ähnlich leicht beweisen.

\section{Satz 3.4}

$S$ ist topologisch transitiv.

Beweis. In jeder offenen Menge $U$ finden wir eine Zahl $x_{0}$, die nach $t$ Iterationen mit einer beliebigen Zahl $y \in[0,1]$ übereinstimmt. Die Zahl $x_{0}$ wird wie folgt konstruiert: Man nehme ein beliebiges Element von $U$, schneide

${ }^{106}$ Deswegen wird die Funktion im Englischen auch shift map genannt. Vgl. Devaney (1989), S. 41. 
dessen Binärentwicklung nach $t$ Stellen ab und ersetze die abgeschnittenen Stellen durch die Binärentwicklung von $y$. Dann gehört die so konstruierte Zahl zu $U$, da $U$ offen ist, und stimmt nach $t$ Iterationen mit $y$ überein.

Und nun noch die letzte Eigenschaft:

\section{Satz 3.5}

Die unter $S$ periodischen Punkte liegen dicht im Einheitsintervall.

Beweis. Ein periodischer Punkt hat eine periodische Binärentwicklung: $x_{0}=0 . \overline{a_{1} a_{2} \ldots a_{t}} \cdot{ }^{107}$ Man nehme einen beliebigen Punkt $x_{0}$ mit einer beliebig kleinen Umgebung. Dann schneide man $x_{0}$ nach $t$ Stellen ab und führe die Binärentwicklung mit den ersten Stellen fort, so dass der so konstruierte Punkt um höchstens $2^{-t}$ von $x_{0}$ entfernt und periodisch ist.

Damit haben wir alle Eigenschaften von Chaos für die Sägezahnfunktion intuitiv einleuchtend nachgewiesen.

\subsubsection{Die Zeltfunktion}

Der nächste Schritt auf dem Weg, Chaos für die logistische Parabel nachzuweisen, ist, die Ergebnisse für die Sägezahnfunktion auf die sogenannte Zeltfunktion zu übertragen ${ }^{108}$, die wie folgt definiert ist:

$$
T:[0,1] \rightarrow[0,1], \quad T(x)= \begin{cases}2 x & \text { für } x<0,5 \\ -2 x+2 & \text { für } x \geq 0,5\end{cases}
$$

Damit wird anhand eines konkreten Beispiels aufgezeigt, wie die Übertragung dynamischer Eigenschaften von einem System auf ein anderes funktioniert. Der Graph ist in Abbildung 3.7 dargestellt.

Wir nutzen folgende Ersetzungseigenschaft aus:

\section{Lemma 3.6}

Für $T$ und $S$ gilt die Identität

$$
T^{t}(x)=T\left(S^{t-1}(x)\right) \text { für alle } x \in[0,1] .
$$

$\overline{107}$ Der Querstrich über $a_{1} a_{2} \ldots a_{t}$ bedeutet, dass sich die Ziffernfolge ad infinitum wiederholt.

${ }^{108}$ Vgl. zu diesem Abschnitt Peitgen, Jürgens und Saupe (1994), S. 95ff. 


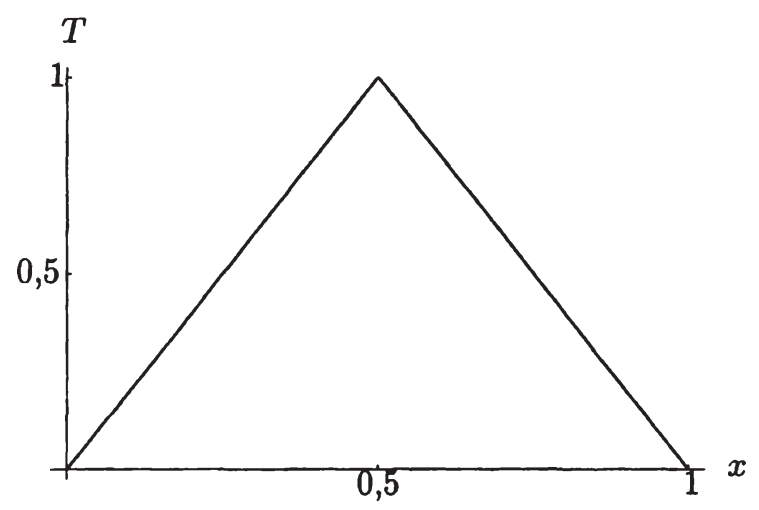

Abbildung 3.7: Graph der Zeltfunktion

Beweis. Man erhält durch einfaches Nachrechnen die Identität $T \circ T=T \circ S$. Durch Anwenden von $T$ auf beiden Seiten ergibt sich $T \circ T \circ T=T \circ T \circ S$, woraus dann sofort $T \circ T \circ T=T \circ S \circ S$ folgt. Die Behauptung erschließt sich daraus durch Induktion.

Mit Gleichung (3.7) ermitteln wir periodische Punkte von $T$, indem wir auf einen periodischen Punkt $w_{0}$ der Sägezahnfunktion einmal $T$ anwenden. Das Ergebnis $x_{0}=T\left(w_{0}\right)$ ist ein periodischer Punkt der Zeltfunktion. Es ist nämlich

$$
x_{t}=T^{t}\left(x_{0}\right)=T^{t}\left(T\left(w_{0}\right)\right)=T^{t+1}\left(w_{0}\right)=T\left(S^{t}\left(w_{0}\right)\right)=T\left(w_{0}\right)=x_{0} .
$$

Beim vierten Gleichheitszeichen wurde von der Ersetzungseigenschaft Gebrauch gemacht. $x_{0}$ ist also tatsächlich ein Punkt der Periode $t$.

Um die Eigenschaften von Chaos nachzuweisen, benutzen wir auch für die Zeltfunktion eine Binärdarstellung. Sei $x=0 . a_{1} a_{2} a_{3} \ldots$ die binäre Form einer Zahl $x \in[0,1]$. Dann gilt:

$$
T(x)= \begin{cases}0 . a_{2} a_{3} a_{4} \ldots & \text { für } x<0,5 \\ 0 . a_{2}^{*} a_{3}^{*} a_{4}^{*} \ldots & \text { für } x \geq 0,5\end{cases}
$$

mit der dualen binären Ziffer

$$
a^{*}= \begin{cases}1 & \text { für } a=0 \\ 0 & \text { für } a=1\end{cases}
$$

Wir zeigen nun 


\section{Satz 3.7}

Die Zeltfunktion weist alle Eigenschaften von Chaos gemäß Definition 3.7 auf.

Beweis.

(i) Dichte periodische Punkte

Der Punkt $w=0 . \overline{0 a_{1} \ldots a_{n}}<1 / 2$ ist periodisch unter $S$. Weiter ist $x=T(w)=S(w)=0 . \overline{a_{1} \ldots a_{n} 0}$. Damit ist $x$ ein periodischer Punkt der Periode $t+1$, dessen erste $t$ Ziffern beliebig sind, so dass in einer Umgebung jedes Punktes des Einheitsintervalls ein periodischer Punkt zu finden ist.

(ii) Sensitivität

Wir wählen einen Punkt $z_{0}$ in der Nähe von $x_{0}$, so dass $\left|z_{0}-x_{0}\right| \leq 2^{-t}$. Dazu konstruieren wir $z_{0}$, indem wir von $x_{0}$ an der $t+1$-sten Stelle die binäre Ziffer umkehren. Nun behaupten wir, dass $\left|z_{t}-x_{t}\right|=1 / 2$. Mit der Ersetzungseigenschaft (3.7) haben wir $T^{t}\left(x_{0}\right)=T\left(0 . a_{t} a_{t+1} a_{t+2} \ldots\right)$ und $T^{t}\left(z_{0}\right)=T\left(0 . a_{t} a_{t+1}^{*} a_{t+2} \ldots\right)$. Für $a_{t}=0$ stimmen $T$ und $S$ überein, so dass $x_{t}=0 . a_{t+1} a_{t+2} a_{t+3} \ldots$ und $z_{t}=0 . a_{t+1}^{*} a_{t+2} a_{t+3} \ldots$ Damit stehen an den ersten Stellen der Binärentwicklungen duale Ziffern, d.h. der Abstand ist tatsächlich $1 / 2$. Für $a_{t}=1$ erhält man $x_{t}=0 . a_{t+1}^{*} a_{t+2}^{*} a_{t+3}^{*} \cdots$ und $z_{t}=0 . a_{t+1} a_{t+2}^{*} a_{t+3}^{*} \ldots$, so dass der Abstand ebenfalls $1 / 2$ beträgt. Damit ist Sensitivität nachgewiesen.

(iii) Transitivität

Gegeben zwei kleine Teilintervalle $I, J$ des Einheitsintervalls, sei $t$ so groß, dass alle Zahlen, deren Binärentwicklung mit $a_{1} \ldots a_{t}$ beginnt, in $I$ liegen und alle Zahlen mit $b_{1} \ldots b_{t}$ in $J$. Wir konstruieren einen Punkt $x_{0} \in I$, dessen $t$-te Iterierte in $J$ liegt. Falls $a_{t}=0$, dann wählen wir $x_{0}=0 . a_{1} \ldots a_{t} b_{1} \ldots b_{t}$, und es ist $x_{t}=T\left(0.0 b_{1} \ldots b_{t}\right)=0, b_{1} \ldots b_{t} \in J$. Wenn $a_{t}=1$ ist, wähle man $x_{0}=0 . a_{1} \ldots a_{t} b_{1}^{*} \ldots b_{t}^{*}$ und erhält $x_{t}=$ $T\left(0.1 b_{1}^{*} \ldots b_{t}^{*}\right)=0 . b_{1} \ldots b_{t} 111 \ldots \in J$.

Die Beweisidee beruht also jeweils darauf, dass man die Eigenschaften der Sägezahnfunktion und die Ersetzungseigenschaft benutzt. 


\subsubsection{Die logistische Parabel}

Die Ergebnisse für die Zeltfunktion lassen sich nun auf die logistische Parabel

$$
f:[0,1] \rightarrow[0,1], \quad x \mapsto 4 x(1-x)
$$

übertragen. ${ }^{109}$ Die Äquivalenz wird durch die Koordinatentransformation

$$
x^{\prime}=h(x)=\sin ^{2}\left(\frac{\pi x}{2}\right)
$$

hergestellt. Dadurch wird das Einheitsintervall injektiv auf sich selbst abgebildet, so dass die transformierten Bahnen der Zeltfunktion den Bahnen der logistischen Parabel entsprechen. Dieser Zusammenhang wird in dem folgenden Lemma präzisiert:

\section{Lemma 3.8}

Für alle $t \in \mathbb{N}$ und $x \in[0,1]$ gilt die Funktionalgleichung

$$
f^{t}(h(x))=h\left(T^{t}(x)\right)
$$

Beweis. Sei $x_{0} \in[0,1]$ der Anfangswert für die Zeltfunktion und dessen Transformation $y_{0}=x_{0}^{\prime}=\sin ^{2}\left(x_{0} \pi / 2\right)$ der für die logistische Parabel. Die Äquivalenz der beiden Bahnen wird durch den Nachweis der Beziehung

$$
\forall t \in \mathbb{N}: y_{t}=x_{t}^{\prime}=h\left(x_{t}\right)
$$

gezeigt. Wir erhalten für $t=1$

$$
\begin{aligned}
y_{1} & =4 y_{0}\left(1-y_{0}\right) \\
& =4 \sin ^{2}\left(\frac{\pi x_{0}}{2}\right)\left(1-\sin ^{2}\left(\frac{\pi x_{0}}{2}\right)\right) \\
& =4 \sin ^{2}\left(\frac{\pi x_{0}}{2}\right) \cos ^{2}\left(\frac{\pi x_{0}}{2}\right) \\
& =\sin ^{2}\left(\pi x_{0}\right) .
\end{aligned}
$$

Bei der Iteration der Zeltfunktion ist eine Fallunterscheidung notwendig.

Fall 1: $0 \leq x \leq 1 / 2$

$$
x_{1}^{\prime}=h\left(x_{1}\right)=h\left(T\left(x_{0}\right)\right)=h\left(2 x_{0}\right)=\sin ^{2}\left(\pi x_{0}\right)
$$

$\overline{109}$ Vgl. hierzu Peitgen, Jürgens und Saupe (1994), S. $100 \mathrm{ff}$. 
Fall 2: $1 / 2<x \leq 1$

$$
\begin{aligned}
x_{1}^{\prime} & =h\left(x_{1}\right)=h\left(T\left(x_{0}\right)\right)=h\left(2-2 x_{0}\right) \\
& =\sin ^{2}\left(\pi-\pi x_{0}\right)=\sin ^{2}\left(-\pi x_{0}\right)=\sin ^{2}\left(\pi x_{0}\right)
\end{aligned}
$$

In beiden Fällen erhält man das gewünschte Ergebnis $y_{1}=x_{1}^{\prime}$. Der Schluss $y_{t}=x_{t}^{\prime}$ für alle $t$ folgt durch Induktion und wegen $x_{t}^{\prime}=h\left(T^{t}\left(x_{0}\right)\right)$ und $y_{t}=f^{t}\left(h\left(x_{0}\right)\right)$ ist die Behauptung bewiesen.

Um einen periodischen Punkt der logistischen Parabel zu ermitteln, braucht man nur auf einen periodischen Punkt der Zeltabbildung die Transformation $h$ anzuwenden.

Mit der Funktionalgleichung 3.9 beweist man nun den

\section{Satz 3.9}

Die logistische Parabel weist alle Eigenschaften von Chaos gemäß Definition 3.7 auf.

\section{Beweis.}

(i) Dichte periodische Punkte

Sei $y \in[0,1]$. Die periodischen Punkte von $f$ liegen dicht, wenn man zu jedem $y \in[0,1]$ eine Folge periodischer Punkte $\left\langle y_{t}\right\rangle$ finden kann mit $\lim _{t \rightarrow \infty} y_{t}=y .{ }^{110} \mathrm{Da} h$ bijektiv ist, können wir mit den Urbildern $x=h^{-1}(y)$ arbeiten. Unter $T$ liegen die periodischen Punkte dicht, d.h. es gibt eine Folge $\left\langle x_{t}\right\rangle$ mit $\lim _{t \rightarrow \infty} x_{t}=x$, wobei jedes $x_{t}$ die Periode $p_{t}$ besitzt. Daher gilt $\forall t \in \mathbb{N}:\left(T^{p_{t}}\left(x_{t}\right)=x_{t}\right)$. Wegen der Funktionalgleichung $f^{t} \circ h=h \circ T^{t}$ gilt

$$
f^{p_{t}}\left(y_{t}\right)=f^{p_{t}}\left(h\left(x_{t}\right)\right)=h\left(T^{p_{t}}\left(x_{t}\right)\right)=h\left(x_{t}\right)=y_{t},
$$

d.h. $\left\langle y_{t}\right\rangle$ ist eine Folge periodischer Punkte. Aus der Stetigkeit von $h$ folgt dann sofort, dass $\lim _{t \rightarrow \infty} y_{t}=y{ }^{111}$

(ii) Topologische Transitivität

Seien $U, V \in[0,1]$ zwei offene Intervalle und $y \in U$. Es ist zu zeigen, dass

${ }^{110}$ Dann liegen schließlich alle Glieder der Folge $\left\langle y_{t}\right\rangle$ in einer Umgebung von $y$, und da $y$ beliebig ist, finden wir in beliebiger Nähe zu jedem Punkt des Einheitsintervalls einen periodischen Punkt.

111 Eine Funktion $f: X \rightarrow X$ heißt stetig genau dann, wenn für jede Folge $\left\langle x_{t}\right\rangle, x_{t} \in X$ mit $\lim _{t \rightarrow \infty} x_{t}=a$ gilt $\lim _{t \rightarrow \infty} f\left(x_{t}\right)=f(a)$. Vgl. Forster (1992), S.60 ff. 
$f^{t}(y) \in V$. Aus der Stetigkeit von $h$ folgt, dass die Urbilder $A=h^{-1}(U)$ und $B=h^{-1}(V)$ ebenfalls offen sind. Da $T$ topologisch transitiv ist, gibt es ein $x \in A$, so dass $T^{t}(x) \in B$. Wir wählen $y=h(x)$, so dass $y \in U$, und wenden die Funktionalgleichung 3.9 darauf an:

$$
f^{t}(y)=f^{t}(h(x))=h\left(T^{t}(x)\right)
$$

Aus $T^{t}(x) \in B$ folgt dann $h\left(T^{t}(x)\right) \in h(B)=V$. Also ist $f$ topologisch transitiv.

(iii) Sensitivität

Die Sensitivität einer Abbildung lässt sich nicht allein durch die Sensitivität einer äquivalenten Abbildung zeigen. ${ }^{112}$ Hier muss zusätzlich ausgenutzt werden, dass $f$ auf einem kompakten Intervall definiert ist ${ }^{113}$. Wir suchen eine Folge $\left\langle y_{t}\right\rangle$ von Anfangswerten der logistischen Parabel, die gegen einen Punkt $y \in[0,1]$ konvergiert, wobei die Bahnen der Folgenglieder sich von der Bahn von $y$ um eine Sensitivitätskonstante $\epsilon_{f}>0$ unterscheiden. Dann lässt sich nämlich in beliebiger Nähe zu $y$ ein Anfangswert finden, der nach endlich vielen Iterationen eine bestimmte Differenz zur Bahn von $y$ aufweist. Für das Urbild $x=h^{-1}(y)$ lässt sich eine solche Folge finden, da $T$ sensitiv ist, d.h. es existiert eine Folge $\left\langle x_{t}\right\rangle$, so dass für alle Folgenglieder $x_{t}$ eine Zahl $k_{t} \in \mathbb{N}$ existiert mit

$$
\left|T^{k_{t}}\left(x_{t}\right)-T^{k_{t}}(x)\right| \geq \epsilon_{T} .
$$

Wir wählen als Sensitivitätskonstante für $f^{114}$

$$
\epsilon_{f}=\inf \left\{|h(a)-h(b)|:|a-b| \geq \epsilon_{T}, a, b \in[0,1]\right\} .
$$

Aus der strengen Monotonie von $h$ folgt, dass für $\epsilon_{f}$ gemäß obiger Definition nur Elemente mit $|a-b|=\epsilon_{T}$ in Frage kommen. Denn ein gröBerer Abstand von $a$ und $b$ führt auch zu einem größeren Abstand von $h(a)$ und $h(b)$. Also ist $\epsilon_{f}$ das globale Minimum der stetigen Funktion $h(\xi)-h\left(\xi-\epsilon_{T}\right) .{ }^{115}$ Und dieses Minimum findet $\operatorname{sich}$ für $h(\xi)=\sin (\pi \xi / 2)$ an der Stelle $\epsilon_{T}$, d.h. $\epsilon_{f}=h\left(\epsilon_{T}\right)>0 .{ }^{116}$

$\overline{112}$ Für ein Gegenbeispiel siehe Peitgen, Jürgens und Saupe (1994), S. 107.

113 Eine Zahlenmenge $X$ heißt kompakt, wenn jede Folge aus $X$ eine konvergente Teilfolge besitzt, deren Grenzwert wieder zu $X$ gehört. Vgl. Heuser (1994), S. 225.

114 Diese Definition hat den Sinn, folgenden Schluss zu ermöglichen: Wenn für $T$ die Sensitivitätskonstante $\epsilon_{T}$ erreicht wird, erreicht auch $f$ die Sensitivitätskonstante $\epsilon_{f}$. Im Übrigen wird hier davon Gebrauch gemacht, dass $f$ auf dem kompakten Intervall $[0,1]$ definiert ist.

115 Diese Funktion ist auf dem Intervall $\left[\epsilon_{T}, 1\right]$ definiert. Die Stetigkeit ergibt sich aus der Definition als Differenz zweier stetiger Funktionen.

${ }^{116}$ Es handelt sich hierbei um ein Randextremum, das sich nicht mit den Mitteln der Differentialrechnung finden lässt. Dass sich an der Stelle $\xi=1$ ein weiteres globales 
Wir betrachten nun die Folge $\left\langle y_{t}\right\rangle$ mit $y_{t}=h\left(x_{t}\right)$. Die $x_{t}$ sind die Folgenglieder der oben betrachteten Folge von Anfangswerten für die Iteration unter $T$. Aus der Stetigkeit von $h$ folgt unmittelbar, dass mit der Folge $\left\langle x_{t}\right\rangle$ auch $\left\langle y_{t}\right\rangle$ konvergiert, und es gilt:

$$
\lim _{t \rightarrow \infty} y_{t}=\lim _{t \rightarrow \infty} h\left(x_{t}\right)=h(x)=y \text {. }
$$

Damit hat man:

$$
\begin{aligned}
\left|f^{k_{t}}\left(y_{t}\right)-f^{k_{t}}(y)\right| & =\left|f^{k_{t}}\left(h\left(x_{t}\right)\right)-f^{k_{t}}(h(x))\right| \\
& =\left|h\left(T^{k_{t}}\left(x_{t}\right)\right)-h\left(T^{k_{t}}(x)\right)\right| \geq \epsilon_{f} .
\end{aligned}
$$

Hier wurde beim ersten Gleichheitszeichen die Definition der Folge $\left\langle y_{t}\right\rangle$ benutzt, beim zweiten Gleichheitszeichen die Funktionalgleichung $f^{t_{k}} \circ$ $h=h \circ T^{t_{k}}$ und beim Ungleichheitszeichen die Definition von $\epsilon_{f}$ sowie die Gleichung (3.10).

Wir haben also gezeigt, dass in beliebiger Nähe von $y$ ein Punkt $y_{k}$ gefunden werden kann, dessen Bahn nach $t_{k}$ Iterationen um die Sensitivitätskonstante $\epsilon_{f}$ von der Bahn von $y$ abweicht.

\subsubsection{Topologische Konjugation}

Wir haben bisher drei Abbildungen auf Chaos hin untersucht. Im ersten Schritt, der Sägezahnfunktion, war Chaos über die binäre Darstellung sehr leicht nachzuweisen. Bei der Zeltfunktion und der logistischen Parabel hingegen wurde Chaos nicht direkt nachgewiesen, sondern es wurden die Beziehungen zwischen den Abbildungen ausgenutzt. Wichtigstes Hilfsmittel dabei waren Funktionalgleichungen der Form $h \circ f=g \circ h$.

Die Funktion $h$, die das Bindeglied zwischen den Funktionen $f$ und $g$ darstellt, muss gewisse Eigenschaften besitzen. Man nennt solche Funktionen Homöomorphismen: ${ }^{117}$

Minimum befindet, ist hier nicht von Belang. Worauf es ankommt, ist nur, dass ein Zusammenhang zwischen $\epsilon_{f}$ und $\epsilon_{T}$ hergestellt ist, der obendrein sicherstellt, dass $\epsilon_{f}$ positiv ist.

117 Vgl. Devaney (1989), S. 9. 


\section{Definition 3.11}

Eine Abbildung $f: X \rightarrow Y$ heißt Homöomorphismus, wenn gilt: $f$ ist stetig und bijektiv, und die Umkehrabbildung $f^{-1}$ ist stetig ${ }^{118}$.

\section{Definition 3.12}

Seien $X, Y \in \mathbb{R}$ Teilmengen der reellen Zahlen und $f: X \rightarrow X$ und $g: Y \rightarrow Y$ Abbildungen. Dann heißen $f$ und $g$ topologisch konjugiert, wenn $f$ und $g$ stetig sind und ein Homöomorphismus $h: X \rightarrow Y$ existiert, so dass

$$
h(f(x))=g(h(x)) \text { für alle } x \in X .
$$

Der Homöomorphismus $h$ heißt topologische Konjugation.

Es sei angemerkt, dass es sich bei topologischer Konjugiertheit um eine Äquivalenzrelation handelt. ${ }^{119}$ Topologisch konjugierte Abbildungen besitzen in qualitativer Hinsicht dieselben dynamischen Eigenschaften. ${ }^{120}$

Durch eine topologische Konjugation wird die Existenz und Stabilität von periodischen Punkten übertragen und man kann bei Kenntnis der periodischen Punkte von $f$ die entsprechenden Punkte von $g$ mittels der Konjugation $h$ berechnen.

\section{Satz 3.10}

Seien $f: X \rightarrow X$ und $g: Y \rightarrow Y$ Abbildungen, die über den Homöomorphismus $h: X \rightarrow Y$ topologisch konjugiert sind. $f$ habe einen stabilen periodischen Punkt $p$. Dann hat auch $g$ einen stabilen periodischen Punkt $q$ mit $q=h(p)$.

Beweis. Sei $n$ die Primperiode von $p$. Wir folgern induktiv. Sei $p=p_{0}$ und $q=q_{0}$. Die Induktionsverankerung stimmt nach Voraussetzung. Sei nun $q_{t}=g^{t}\left(q_{0}\right)=h\left(p_{t}\right)=f^{t}\left(p_{0}\right)$. Dann gilt:

$$
q_{t+1}=g\left(q_{t}\right)=g\left(h\left(p_{t}\right)\right)=h\left(f\left(p_{t}\right)\right)=h\left(p_{t}\right)
$$

Die Eigenschaft der topologischen Konjugiertheit ist das entscheidende Hilfsmittel beim analytischen Nachweis chaotischen Verhaltens. Denn es wird

118 Die Existenz der Umkehrabbildung ist durch die Bijektivität von $f$ gesichert.

${ }^{119}$ Eine Relation $\sim$ auf einer Menge $X$ heißt Äquivalenzrelation, wenn sie reflexiv, symmetrisch und transitiv ist, d.h. wenn für alle $x, y, z \in X$ gilt: $x \sim x, x \sim y \Rightarrow y \sim x$ und $x \sim y \wedge x \sim z \Rightarrow x \sim z$. Vgl. Fischer (1995), S. 38 .

120 Vgl. Devaney (1989), S. 47. 
sich zeigen, dass sich Chaos durch topologische Konjugation vererbt, d.h. wenn $f$ chaotisch ist und $g$ topologisch konjugiert zu $f$ ist, ist damit auch $g$ chaotisch. Beim Beweis der topologischen Transitivität und der dichten periodischen Punkte der logistischen Parabel in Satz 3.9 wurde auf eben dieses Konzept zurückgegriffen. Die topologische Konjugation war der Homöomorphismus $h(x)=\sin ^{2}(\pi x / 2) \cdot{ }^{121}$

Zwei Punkte sind jedoch auf dem Weg dorthin zu beachten. Zum einen haben wir gesehen, dass sich Sensitivität allein, ohne die beiden anderen Eigenschaften, nicht unbedingt durch eine topologische Konjugation übertragen muss. Im Beweis zu Satz 3.9 hat dies nicht genügt. ${ }^{122}$ Zum anderen existiert kein Homöomorphismus, der Sägezahn- und Zeltfunktion in Beziehung setzen könnte. Existierte ein Homöomorphismus $h$, wäre $S$ wegen $h \circ S=T \circ h \Longleftrightarrow S=h^{-1} \circ T \circ h$ stetig $^{123}$, was aber nicht der Fall ist. Wir haben die Funktionalgleichung $T \circ S=T \circ T$ benutzt; $T$ ist aber nicht injektiv.

Die erste Schwierigkeit lässt sich durch ein Ergebnis lösen, das erst einige Zeit nach Devaneys Definition von Chaos vorgelegt wurde. ${ }^{124}$ Es wurde gezeigt, dass die sensitive Abhängigkeit von den Anfangsbedingungen bereits aus den übrigen beiden Bedingungen folgt, deren Nachweis damit genügt. ${ }^{125}$

Um dieses Ergebnis darstellen zu können, benötigen wir das folgende

\section{Lemma 3.11}

Sei $X$ eine offene Teilmenge von $\mathbb{R}$ und $f: X \rightarrow X$ eine stetige Funktion, die dichte periodische Punkte besitzt. Dann gibt es ein $\epsilon_{0}>0$, so dass für jedes $x \in X$ ein periodischer Punkt $p \in X$ existiert mit der Eigenschaft

$$
\left|f^{t}(p)-x\right| \geq \epsilon_{0} / 2
$$

für alle $t \in \mathbb{N}$.

Beweis. Wir wählen zwei beliebige periodische Punkte $p_{1}$ und $p_{2}$ mit disjunkten Bahnen, d.h. $f^{t}\left(p_{1}\right) \neq f^{\tau}\left(p_{2}\right)$ für alle $t, \tau \in \mathbb{N}$. Die Existenz zweier

$\overline{121}$ Vgl. Peitgen, Jürgens und Saupe (1994), S. 100.

${ }^{122}$ Es sei an dieser Stelle auch nochmals auf das Gegenbeispiel bei Peitgen, Jürgens und Saupe (1994), S. 107, hingewiesen.

${ }^{123}$ Es sei daran erinnert, dass sowohl $T$ als auch der Homöomorphismus $h$ stetig sind. Damit sind auch die Umkehrabbildung $h^{-1}$ und Kompositionen aus diesen Funktionen stetig. Vgl. Forster (1992) S. 63 und 71.

124 Vgl. Banks, Brooks, Cairns, Davis und Stacey (1992).

${ }^{125}$ Es wäre nur konsequent, die Eigenschaft der Sensitivität fortan aus der Definition 3.7 von Chaos zu streichen. Dies ist wohl deshalb bislang noch nicht geschehen, weil es sich bei der Sensitivität um die augenfälligste Eigenschaft handelt. 
solcher Punkte ist dadurch gewährleistet, dass die periodischen Punkte nach Voraussetzung dicht in $X$ liegen und $f$ stetig ist. Nun sei

$$
\left.\epsilon_{0}=\min \left\{\left|f^{t}\left(p_{1}\right)-f^{\tau}\left(p_{2}\right)\right|\right\}: t, \tau \in \mathbb{N}\right\}
$$

und $x \in X$. Die Behauptung ist bewiesen, wenn $x$ zu einer der beiden Bahnen mindestens einen Abstand von $\epsilon_{0} / 2$ hat. Der Beweis erfolgt durch Widerspruch. Angenommen also, das Gegenteil wäre der Fall. Dann gäbe es $t, \tau$ mit

$$
\left|f^{t}\left(p_{1}\right)-x\right|<\epsilon_{0} / 2 \text { und }\left|f^{\tau}\left(p_{2}\right)-x\right|<\epsilon_{0} / 2
$$

Mit der Dreiecksungleichung ${ }^{126}$ kann man folgern:

$$
\begin{aligned}
\left|f^{t}\left(p_{1}\right)-f^{\tau}\left(p_{2}\right)\right| & =\left|f^{t}\left(p_{1}\right)-x+x-f^{\tau}\left(p_{2}\right)\right| \\
& \leq\left|f^{t}\left(p_{1}\right)-x\right|+\left|x-f^{\tau}\left(p_{2}\right)\right| \\
& <\frac{\epsilon_{0}}{2}+\frac{\epsilon_{0}}{2}=\epsilon_{0} .
\end{aligned}
$$

Dies steht aber im Widerspruch zur Definition von $\epsilon_{0}$.

Die Beweisidee des Lemmas beruht also lediglich darauf, dass es periodische Punkte mit disjunkten Bahnen gibt, denen $x$ nicht beiden beliebig nahe liegen kann.

\section{Satz 3.12}

Sei $X$ eine offene Teilmenge von $\mathbb{R}$ und $f: X \rightarrow X$ eine stetige Funktion, die dichte periodische Punkte besitzt und topologisch transitiv ist. Dann zeigt $f$ auch sensitive Abhängigkeit von den Anfangsbedingungen.

Beweis. Wir wählen die Sensitivitätskonstante $\epsilon=\epsilon_{0} / 8$. Sei $J$ eine offene Teilmenge von $X$ und $x \in J$. Wir finden einen periodischen Punkt $p$ in $U=J \cap(x-\epsilon, x+\epsilon)$ mit der Periode $t$. Nach Lemma 3.11 gibt es einen periodischen Punkt $q \in X$, dessen Bahn einen Abstand von mindestens $4 \epsilon=$ $\epsilon_{0} / 2$ von $x$ hat. Wir definieren

$$
\begin{gathered}
W_{\tau}=\left(f^{\tau}(q)-\epsilon, f^{\tau}(q)+\epsilon\right) \cap X, \quad \tau=1, \ldots, t, \\
V=\bigcap_{\tau=1}^{t} f^{-\tau}\left(W_{\tau}\right) .
\end{gathered}
$$

${ }^{126}$ Vgl. Fischer (1995), S. 255. 
Die so konstruierte Menge ${ }^{127} V$ ist nicht leer, da $q \in V$ ist. Außerdem ist sie offen. Dazu stellen wir fest, dass alle $W_{\tau}$ offen sind. Wegen der Stetigkeit von $f$ sind auch die Urbilder $f^{-\tau}\left(W_{\tau}\right)$ offen und der Durchschnitt endlich vieler offener Mengen ist wieder offen. ${ }^{128}$

Wir wählen ein $y \in U$, für das gilt: $f^{k}(y) \in V$. Die Existenz eines solchen $y$ ist durch die topologische Transitivität von $f$ gewährleistet. Sei $j$ der ganzzahlige Anteil von $1+\frac{k}{t}$, so dass $1 \leq t \cdot j-k \leq t$. $y$ wurde so gewählt, dass

$$
f^{t \cdot j}(y)=f^{t \cdot j-k}\left(f^{k}(y)\right) \in f^{t \cdot j-k}(V) .
$$

Damit ist $f^{t \cdot j}(y) \in W_{t \cdot j-k}$. Wegen $f^{t \cdot j}(p)=p$ kann man mit der Dreiecksungleichung schließen:

$$
\begin{aligned}
\left|f^{t \cdot j}(p)-f^{t \cdot j}(y)\right| & =\left|p-f^{t \cdot j}(y)\right| \\
& =\left|x-f^{t \cdot j-k}(q)+f^{t \cdot j-k}(q)-f^{t \cdot j}(y)+p-x\right| \\
& \geq\left|x-f^{t \cdot j-k}(q)\right|-\left|f^{t \cdot j-k}(q)-f^{t \cdot j}(y)\right|-|p-x| .
\end{aligned}
$$

Dazu haben wir

$$
\begin{gathered}
p \in(x-\epsilon, x+\epsilon), \\
f^{t \cdot j}(y) \in\left(f^{t \cdot j-k}(q)-\epsilon, f^{t \cdot j-k}(q)+\epsilon\right) \cap X .
\end{gathered}
$$

Daraus ergibt sich

$$
\left|f^{t \cdot j}(p)-f^{t \cdot j}(y)\right| \geq 4 \epsilon-\epsilon-\epsilon=2 \epsilon .
$$

Damit muss entweder $\left|f^{t \cdot j}(x)-f^{t \cdot j}(y)\right| \geq \epsilon \operatorname{oder}\left|f^{t \cdot j}(x)-f^{t \cdot j}(p)\right| \geq \epsilon$ gelten. Anderenfalls käme man über die Dreiecksungleichung zu

$$
\begin{aligned}
\left|f^{t \cdot j}(p)-f^{t \cdot j}(y)\right| & =\left|f^{t \cdot j}(p)-f^{t \cdot j}(x)+f^{t \cdot j}(x)-f^{t \cdot j}(y)\right| \\
& =\left|f^{t \cdot j}(p)-f^{t \cdot j}(x)\right|+\left|f^{t \cdot j}(x)-f^{t \cdot j}(y)\right| \\
& <2 \epsilon .
\end{aligned}
$$

Da $p, y \in U \subset J$ ist, ist nun klar, dass die $t \cdot j$-te Iterierte von $f$ an der Stelle $p$ oder $y$ von der $t \cdot j$-ten Iterierten von $x$ um mindestens $\epsilon$ abweicht.

Die zweite Schwierigkeit zwingt dazu, das Konzept der topologischen Konjugation $\mathrm{zu}$ modifizieren. ${ }^{129}$

${ }^{127}$ Die Konstruktion besagt, dass in $V$ diejenigen Anfangswerte enthalten sind, deren $t$ Iterierte von den entsprechenden Iterierten von $q$ um nicht mehr als $\epsilon$ abweichen, oder anders ausgedrückt: Die Bahnen aller in $V$ enthaltenen Anfangswerte haben von der Bahn von $q$ einen Abstand von höchstens $\epsilon$.

128 Vgl. Heuser (1994), S. 218.

129 Vgl. Peitgen, Jürgens und Saupe (1994), S. 113. 


\section{Definition 3.13}

Seien $X, Y \subset \mathbb{R}$ und $f: X \rightarrow X$ und $g: Y \rightarrow Y$ Abbildungen. Dann heißt $g$ genau dann topologisch semikonjugiert zu $f$, wenn eine stetige, surjektive Abbildung $h: X \rightarrow Y$ existiert, so dass die Funktionalgleichung

$$
h(f(x))=g(h(x))
$$

für alle $x \in X$ erfüllt ist.

Diese Definition ist in zwei Punkten schwächer als die der topologischen Konjugation 3.12. Zum einen wird für $f$ und $g$ keine Stetigkeit gefordert; zum anderen muss $h$ kein Homöomorphismus sein, da Injektivität nicht gefordert wird. Mit dem Konzept der topologischen Semikonjugation lässt sich also auch die Beziehung $T \circ T=T \circ S$ erfassen. Das Problem war ja gerade, dass $T$ nicht injektiv ist. Andererseits ist die topologische Semikonjugiertheit keine Äquivalenzrelation. Das liegt daran, dass für $h$ womöglich keine Umkehrabbildung existiert.

Wir benötigen noch das folgende

\section{Lemma 3.13}

Gegeben seien die Abbildungen $f: X \rightarrow X, g: Y \rightarrow Y$, und $h: X \rightarrow Y$. Aus der Funktionalgleichung $h(f(x))=g(h(x))$ folgt $h\left(f^{n}(x)\right)=g^{n}(h(x))$ für alle $n \in \mathbb{N}$.

Beweis. Aus $h \circ g=g \circ h$ folgt $g \circ h \circ f=g \circ g \circ h$. Wir wenden auf die linke Seite $g \circ h=h \circ f$ an und erhalten $f \circ f^{2}=g^{2} \circ h$. Die Behauptung folgt daraus durch Induktion nach $n$.

Mit dem folgenden Satz wird nun gezeigt, dass die Herleitung der chaotischen Eigenschaften von $T$ aus denen von $S$ ebenfalls vor einem allgemeinen Hintergrund gesehen werden kann. ${ }^{130}$

\section{Satz 3.14}

Sei $g: Y \rightarrow Y$ semikonjugiert zu $f: X \rightarrow X$ über eine stetige, surjektive Funktion $h$. Außerdem sei $f$ topologisch transitiv und habe dichte periodische Punkte. Dann ist auch $g$ topologisch transitiv und hat dichte periodische Punkte.

$\overline{130}$ Vgl. Peitgen, Jürgens und Saupe (1994), S. 114. 


\section{Beweis.}

(i) Dichte periodische Punkte

Sei $y \in Y$ und $x$ das Urbild von $y$ unter $h$, d.h. $h(x)=y$. Dann gibt es eine konvergente Folge $\left\langle x_{t}\right\rangle$ periodischer Punkte von $f \operatorname{mit} \lim _{t \rightarrow \infty}\left\langle x_{t}\right\rangle=$ $x$ und $f^{n_{t}}\left(x_{t}\right)=x_{t}$. Die Behauptung ist, dass für die Folge $\left\langle y_{t}\right\rangle$ gilt: $\lim _{t \rightarrow \infty}\left\langle y_{t}\right\rangle=y$ und $g^{n_{t}}\left(y_{t}\right)=y_{t}$. Der erste Teil der Behauptung ergibt sich unmittelbar aus der Stetigkeit von $h$, der zweite ergibt sich aus Lemma 3.13. Es ist

$$
g^{n_{t}}\left(y_{t}\right)=g^{n_{t}}\left(h\left(x_{t}\right)\right)=h\left(f^{n_{t}}\left(x_{t}\right)\right)=h\left(x_{t}\right)=y_{t} .
$$

(ii) Topologische Transitivität

Seien $U$ und $V$ offene Mengen in $Y$. Es wird behauptet, dass ein $y \in$ $U$ und ein $t>0$ existieren mit $g^{t}(y) \in V$. Wir nehmen die offenen Urbilder $A=h^{-1}(U)$ und $B=h^{-1}(V)$. Nach Voraussetzung existiert ein $x \in A$, so dass $f^{t}(x) \in B$. Auf $y=h(x)$ wird wiederum Lemma 3.13 angewandt:

$$
g^{t}(y)=g^{t}(h(x))=h\left(f^{t}(x)\right) \in h(B)=V .
$$

Das Ergebnis des Abschnitts fassen wir noch zusammen in

\section{Corollar 3.15}

Sei $f$ eine chaotische Abbildung und $g$ topologisch semikonjugiert zu $f$. Dann ist auch $g$ chaotisch.

Beweis. Das Corollar ergibt sich unmittelbar aus Lemma 3.11 mit Satz 3.14 .

Der Zusammenhang zwischen den bisher behandelten Abbildungen, der Sägezahnfunktion, der Zeltfunktion und der logistischen Parabel, lässt sich auch in dem folgenden kommutativen Diagramm darstellen:

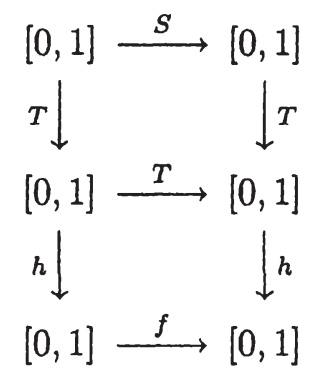




\subsection{Zur Numerik von Chaos}

\subsubsection{Das Beschattungslemma}

Numerische Experimente an Computern zeigen, dass das berechnete Verhalten chaotischer Systeme vom theoretisch zu erwartenden Verhalten abweicht. ${ }^{131}$ Dies liegt daran, dass Computer nur mit einer begrenzten Rechengenauigkeit arbeiten. Sie speichern reelle Zahlen im Binärsystem und schneiden nach einer gewissen Zahl von Stellen einfach ab. Zahlen mit unendlicher Binärentwicklung lassen sich nicht exakt auf dem Computer darstellen, und damit startet die Iteration mit einem geringfügig falschen Wert. Die Sensitivität des Systems führt nach einer gewissen Anzahl von Iterationen dazu, dass die berechnete und die theoretische Bahn signifikant voneinander abweichen.

Unter diesen Umständen ist es berechtigt zu fragen, welche Aussagekraft numerische Experimente mit einem beschränkt genauen Rechner überhaupt haben. Die überraschende Antwort gibt das sogenannte Beschattungslemma: Obwohl die berechnete Bahn grundsätzlich falsch ist, gibt es eine Bahn desselben Systems, die von der berechneten gut approximiert wird. Oder anders ausgedrückt: Wir können zwar nicht die Bahn eines gegebenen Startwertes $x_{0}$ berechnen, aber wir wissen, dass die berechnete Bahn tatsächlich - zumindest näherungsweise - existiert, wenn wir auch deren Startwert $z_{0}$ nicht kennen. Dies veranschaulicht Abbildung 3.8.

Das Beschattungslemma wird hier nur für die Sägezahnfunktion formuliert. Aufgrund der topologischen Konjugiertheit zur logistischen Parabel ist klar, dass es auch für diese gilt. In der Tat behält das Beschattungslemma in vielen chaotischen Systemen seine Gültigkeit ${ }^{132}$, so dass der Beweis gewissermaßen exemplarischen Charakter hat.

Zuvor führen wir noch eine bequemere Schreibweise für die Sägezahnfunktion ein, die auch im Dezimalsystem die Fallunterscheidung erspart. Dazu benötigen wir den Operator Frac $(x)$, der den nicht-ganzzahligen Anteil einer Zahl $x$ bezeichnet:

$$
\operatorname{Frac}(x)=x-k, \quad \text { wenn } k \leq x<k+1, k \in \mathbb{Z} .
$$

$\overline{131}$ Vgl. Peitgen, Jürgens und Saupe (1992), S. 61ff.

132 Vgl. Peitgen, Jürgens und Saupe (1994), S. 125. 

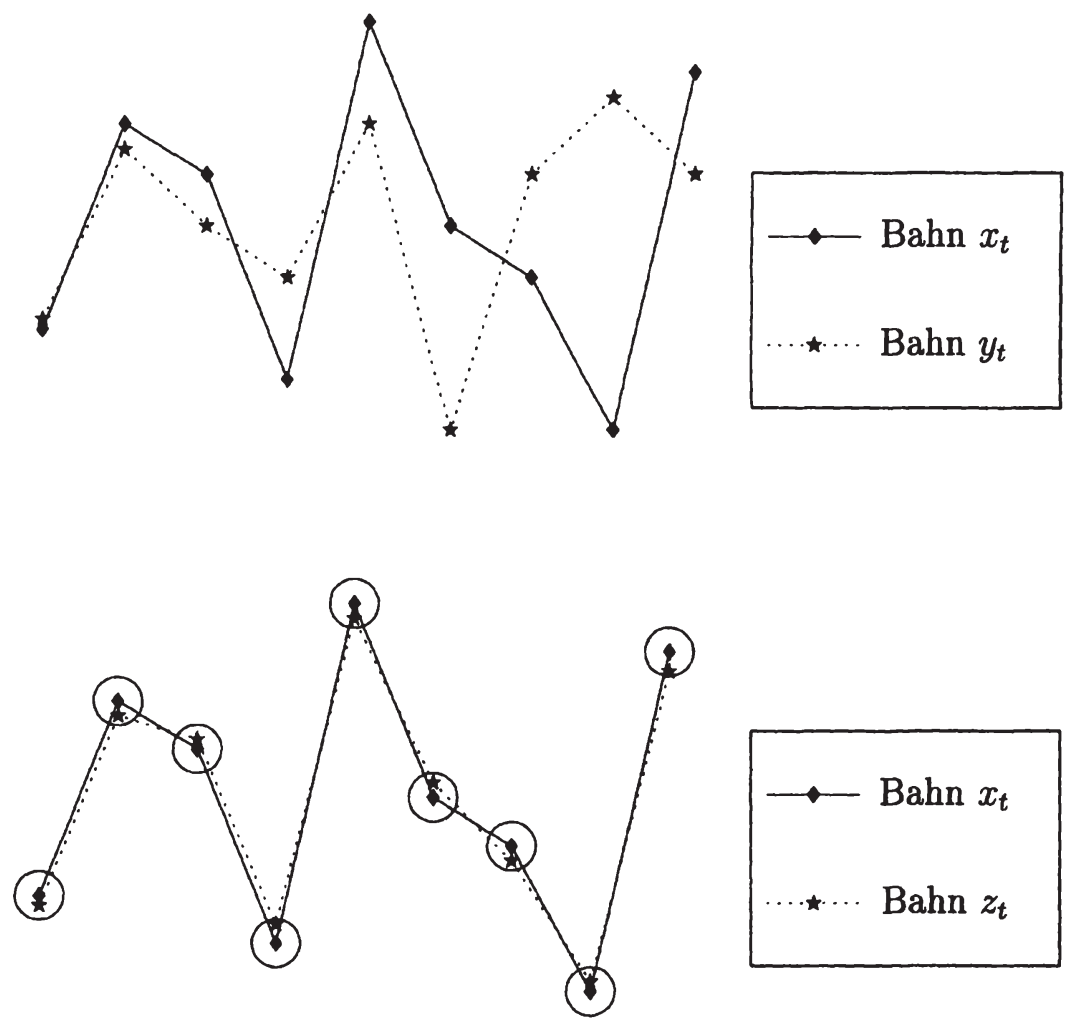

Bahn $x_{t}$

Bahn $z_{t}$

In Anlehnung an: Peitgen, Jürgens und Saupe (1994), S. 121

Abbildung 3.8: Beschattungslemma

Der Frac-Operator hat folgende Eigenschaften, die sich leicht nachrechnen lassen $^{133}$ : Sei $m \in \mathbb{Z}$. Dann gilt

$$
\begin{gathered}
\operatorname{Frac}(x+m)=\operatorname{Frac}(x) \\
\operatorname{Frac}(m \cdot x)=\operatorname{Frac}(m \cdot \operatorname{Frac}(x)) .
\end{gathered}
$$

Mit dem Frac-Operator kann man die Sägezahnfunktion schreiben als

$$
S(x)=\operatorname{Frac}(2 x) \text { für } 0 \leq x<1 .
$$

Die Iteration lässt sich nun in geschlossener Form darstellen. $\overline{133}$ Vgl. Peitgen, Jürgens und Saupe (1994), S. 84f. 


\section{Lemma 3.16}

Für die $t$-te Iterierte der Sägezahnfunktion gemäß Gleichung 3.11 gilt:

$$
x_{t}=S^{t}\left(x_{0}\right)=\operatorname{Frac}\left(2^{t} x_{0}\right) .
$$

Beweis. Der Beweis erfolgt durch Induktion. Die Induktionsvoraussetzung ist per Definition erfüllt. Es bleibt der Schluss von $t$ auf $t+1$. Nach Induktionsannahme ist

$$
x_{t}=\operatorname{Frac}\left(2^{t} x_{0}\right) .
$$

Sei $k \in \mathbb{Z}$ diejenige ganze Zahl mit $k \leq 2^{t} x_{0}<k+1$. Dann berechnen wir

$$
\begin{aligned}
x_{t+1} & =\operatorname{Frac}\left(2 x_{t}\right)=\operatorname{Frac}\left(2 \operatorname{Frac}\left(2^{t} x_{0}\right)\right) \\
& =\operatorname{Frac}\left(2\left(2^{t} x_{0}-k\right)\right)=\operatorname{Frac}\left(2^{t+1} x_{0}-2 k\right) \\
& =\operatorname{Frac}\left(2^{t+1} x_{0}\right) .
\end{aligned}
$$

Wir formulieren nun das Beschattungslemma für die Sägezahnfunktion.

\section{Lemma 3.17}

Gegeben sei ein exakter Anfangswert $x_{0}$ und die zugehörige Bahn $x_{\tau+1}=$ $S\left(x_{\tau}\right), \tau \in \mathbb{N}$. Bei jeder Iteration darf ein Fehler $\epsilon_{\tau}$ auftreten, so dass ein fehlerhafter Anfangswert

$$
y_{0}=x_{0}+\epsilon_{0}
$$

und eine fehlerhafte Bahn mit

$$
y_{\tau}=\operatorname{Frac}\left(2 y_{\tau-1}+\epsilon_{\tau}\right)
$$

entstehen. Es gebe ein $\epsilon>0$, so dass $\left|\epsilon_{\tau}\right| \leq \epsilon^{134}$ Dann existiert ein Anfangswert $z_{0}$, für dessen Bahn gilt:

$$
\left|z_{\tau}-y_{\tau}\right| \leq \epsilon
$$

Beweis. Die $\tau$-te Iterierte der fehlerhaften Bahn lautet unter Verwendung von Lemma 3.16

$$
y_{\tau}=\operatorname{Frac}\left(2^{\tau} x_{0}+\sum_{i=0}^{\tau} 2^{\tau-i} \epsilon_{i}\right) .
$$

${ }^{134}$ Dass die Fehler durch eine positive Konstante beschränkt sind, ist die einzige nötige einschränkende Annahme. Die Voraussetzungen des Beschattungslemmas werden von Computersimulationen erfüllt: In jeder Iteration tritt ein Rundungsfehler auf, der aber immer von der Rechengenauigkeit begrenzt wird. 
Wir wählen $z_{0}$ so, dass $z_{t}=y_{t}$, also

$$
z_{0}=\operatorname{Frac}\left(x_{0}+\sum_{i=0}^{t} 2^{-i} \epsilon_{i}\right)
$$

und erhalten

$$
z_{\tau}=\operatorname{Frac}\left(2^{\tau} x_{0}+\sum_{i=0}^{t} 2^{\tau-i} \epsilon_{i}\right) .
$$

Die Differenz $\Delta_{\tau}$ der Argumente des Frac-Operators ergibt sich zu

$$
\Delta_{\tau}=\sum_{i=\tau+1}^{t} 2^{\tau-i} \epsilon_{i}
$$

Dafür lässt sich eine obere Grenze angeben:

$$
\left|\Delta_{\tau}\right| \leq \sum_{i=\tau+1}^{t} 2^{\tau-i}\left|\epsilon_{i}\right| \leq \sum_{i=\tau+1}^{t} 2^{\tau-i} \epsilon<\epsilon .
$$

Die Nähe der Argumente überträgt sich auf die Werte des Frac-Operators, wenn man die Abstandsmessung nicht auf dem Einheitsintervall, sondern auf dem Einheitskreis durchführt. Statt des Betrages verwendet man dabei die Metrik $\min \{|x-y|, 1-|x-y|\}$, wobei $x, y \leq 1 .{ }^{135}$ Anderenfalls kann es bei einzelnen Iterationen vorkommen, dass das Argument von $y_{\tau}$ etwas unterhalb einer ganzen Zahl liegt und das Argument von $z_{\tau}$ etwas oberhalb, so dass $\left|z_{\tau}-y_{\tau}\right| \approx 1$. Der Grund liegt in der Unstetigkeit des Frac-Operators an ganzzahligen Punkten. Streng genommen muss die Behauptung des Beschattungslemmas in diesem Sinne modifiziert werden. ${ }^{136}$

Die Behauptung für alle Zahlen $\tau \in \mathbb{N}$ wird durch den Grenzübergang $t \rightarrow \infty$ bewiesen. Man erhält

$$
\left|\Delta_{\tau}\right| \leq \sum_{i=\tau+1}^{\infty} 2^{\tau-i}\left|\epsilon_{i}\right| \leq \sum_{i=\tau+1}^{\infty} 2^{\tau-i} \epsilon=\epsilon
$$

und der Rest folgt analog.

135 Vgl. Alligood, Sauer und Yorke (1997), S. 27. Eine Metrik $d$ ordnet allgemein zwei Elementen $X$ und $Y$ einer Menge eine positive reelle Zahl zu mit folgenden Eigenschaften: $d(X, Y) \geq 0, d(X, Y)=0 \Longleftrightarrow X=Y, d(X, Y)=d(Y, X)$ und $d(X, Y)+d(Y, Z) \leq d(X, Z)$. Die letzte Eigenschaft heißt Dreiecksungleichung. Mit Hilfe einer solchen Metrik werden Abstandsmessungen in beliebigen Räumen möglich. Vgl. Heuser (1994), S. 85.

${ }^{136}$ Vgl. Peitgen, Jürgens und Saupe (1994), S. 124. 
Die Quintessenz des Beschattungslemmas ist, dass die Dynamik eines chaotischen Systems so reichhaltig ist, dass sich innerhalb des $\epsilon$-Schattens einer fehlerbehafteten Bahn eine exakte Bahn befindet. Dies ist umso erstaunlicher, als die genaue Größe der Fehler $\epsilon_{\tau}$ gar keine Rolle spielt, solange sie nur beschränkt bleibt.

Dennoch bietet dies keinen Ausweg aus dem Problem der Sensitivität. Denn es gibt nach wie vor keine Möglichkeit, aus einem gegebenen Anfangswert das Langzeitverhalten des Systems zu prognostizieren. Im Gegenteil können auch scheinbar willkürliche Prognosen von dem Modell gestützt werden. Der Nutzen des Beschattungslemmas liegt einzig darin, dass die statistischen Eigenschaften eines chaotischen Systems, die durch numerische Experimente ermittelt werden, tatsächlich aussagekräftig sind.

\subsubsection{Lyapunov-Exponenten}

Der nichtlineare Charakter chaotischer Systeme macht eine allgemeine analytische Behandlung oft unmöglich. Wenn sich aber Chaos nicht direkt nachweisen lässt, stellt sich die Frage, ob man nicht wenigstens durch numerische Untersuchungen Hinweise auf chaotisches Verhalten entdecken kann. Ein sehr aussagekräftiges Indiz ist der Lyapunov-Exponent eines dynamischen Systems, der es erlaubt, die Stärke der sensitiven Abhängigkeit von den Anfangsbedingungen zu messen. Sensitivität sorgt dafür, dass ein beliebig kleiner Fehler durch Iteration immer größer wird. Als Idee für ein Maß der Sensitivität bietet sich die Geschwindigkeit des Fehlerwachstums an. ${ }^{137}$

Sei $\epsilon_{0}$ der Fehler, mit dem der Anfangswert behaftet ist. Die Iteration beginnt also statt mit dem exakten Wert $x_{0}$ mit dem fehlerhaften Wert $\tilde{x}_{0}=$ $x_{0}+\epsilon_{0}$. Die gesamte Fehlerverstärkung ist damit

$$
\left|\frac{\epsilon_{t}}{\epsilon_{0}}\right|=\prod_{\tau=1}^{t}\left|\frac{\epsilon_{\tau}}{\epsilon_{\tau-1}}\right| .
$$

In einem linearen System $x \mapsto c \cdot x$ wächst der Fehler in jeder Iteration um den Faktor $c$, d.h.

$$
\left|\frac{\epsilon_{t}}{\epsilon_{0}}\right|=c^{t}
$$

Dies formen wir um zu

$$
\ln c=\frac{1}{t} \ln \left|\frac{\epsilon_{t}}{\epsilon_{0}}\right| .
$$

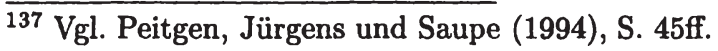


Diese Formel kann man auf beliebige Iteratoren anwenden. Man nähert also dort das Fehlerwachstum $\left|\epsilon_{t} / \epsilon_{0}\right|$ durch ein geometrisches Mittel an, als wäre es von einer linearen Transformation erzeugt worden.

Es taucht jedoch ein Problem auf. Spielt sich die Dynamik auf einem begrenzten Bereich ab, kann der Fehler nach einer gewissen Anzahl von Iterationen nicht mehr weiter wachsen. So legen numerische Experimente mit der logistischen Parabel $x \mapsto 4 x(1-x)$ den Schluss nahe, dass sich der Fehler bei jeder Iteration im Durchschnitt verdoppelt. ${ }^{138}$ Nun ist der Wertebereich auf das Einheitsintervall beschränkt, so dass nach einer gewissen Anzahl von Iterationen der Fehler so groß ist, dass eine Verdopplung schon deshalb ausgeschlossen ist. Diese Anzahl ist selbst bei sehr kleinen Anfangsfehlern zu klein, um daraus verlässliche numerische Aussagen ableiten zu können. Es gibt jedoch einen Ausweg.

Wir versuchen, die einzelnen Fehlerverstärkungsfaktoren $\left|\epsilon_{\tau} / \epsilon_{\tau-1}\right|$ abzuschätzen und setzen dies in die rechte Seite von Gleichung 3.13 ein. Es ergibt sich:

$$
\frac{1}{t} \ln \left|\frac{\epsilon_{t}}{\epsilon_{0}}\right|=\frac{1}{t} \sum_{\tau=1}^{t} \ln \left|\frac{\epsilon_{\tau}}{\epsilon_{\tau-1}}\right| .
$$

Die Idee besteht darin, auszunutzen, dass der Fehlerverstärkungsfaktor $\epsilon_{\tau} / \epsilon_{\tau-1}$ von der Größe des Fehlers $\epsilon_{\tau-1}$ nur unwesentlich beeinflusst wird. Dies sei am Beispiel der logistischen Parabel $x \mapsto 4 x(1-x)$ verdeutlicht. Der fehlerhafte Wert sei $\tilde{x}_{\tau}=x_{\tau}+\epsilon_{\tau}$. Dann ist

$$
\begin{aligned}
\epsilon_{\tau} & =\tilde{x}_{\tau}-x_{\tau}=f\left(\tilde{x}_{\tau-1}\right)-f\left(x_{\tau-1}\right) \\
& =4\left(x_{\tau-1}+\epsilon_{\tau-1}\right)\left(1-x_{\tau-1}-\epsilon_{\tau-1}\right)-4 x_{\tau-1}\left(1-x_{\tau-1}\right) \\
& =4 \epsilon_{\tau-1}\left(1-2 x_{\tau-1}\right)-4 \epsilon_{\tau-1}^{2}
\end{aligned}
$$

und

$$
\frac{\epsilon_{\tau}}{\epsilon_{\tau-1}}=4\left(1-2 x_{\tau-1}\right)-4 \epsilon_{\tau-1} \text {. }
$$

Dies bedeutet nichts anderes, als dass etwa eine Halbierung des Ausgangsfehlers auch den Folgefehler ungefähr halbiert. Man kann diesen Fakt ausnutzen, indem man statt des echten Fehlers $\epsilon_{\tau-1}$, der für genügend großes $\tau$ unbrauchbar groß wird, einfach irgendeinen konstanten kleinen Fehler $\epsilon$ einsetzt. Dadurch braucht man sich um das Wachstum des Fehlers und die Anzahl der zulässigen Iterationen keine Gedanken zu machen, es sind beliebig viele Iterationen und damit auch Grenzwertberechnungen möglich. Als

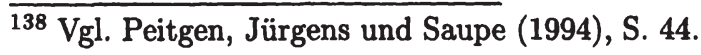


Abschätzung der Fehlerverstärkungsfaktoren erhält man

$$
\left|\frac{\epsilon_{\tau}}{\epsilon_{\tau-1}}\right| \approx \frac{\left|\tilde{\epsilon}_{\tau}\right|}{\epsilon}=\frac{\left|f\left(x_{\tau}+\epsilon\right)-f\left(x_{\tau}\right)\right|}{\epsilon} .
$$

Der Lyapunov-Exponent eines Systems kann im Experiment durch folgendes Vorgehen geschätzt werden. ${ }^{139}$ Man wählt einen Fehlerwert $\epsilon$, etwa 0,001 , sowie einen Anfangswert $x_{0}$ und berechnet $t$ zugehörige Iterationen. Daraus kann man die Fehlerabschätzungen $\tilde{\epsilon}_{\tau}=f\left(x_{\tau}+\epsilon\right)-f\left(x_{\tau}\right)$ ermitteln und logarithmieren. Durch Aufsummieren und Dividieren durch $t$ ergibt sich dann die Abschätzung gemäß Formel 3.14. ${ }^{140}$

Mathematisch exakter fasst man den Lyapunov-Exponenten aus dem Fehlerterm

$$
\frac{\epsilon_{\tau}}{\epsilon_{\tau-1}}=\frac{f\left(x_{\tau-1}+\epsilon_{\tau-1}\right)-f\left(x_{\tau-1}\right)}{\epsilon_{\tau-1}}
$$

durch Grenzübergang ${ }^{141}$ :

$$
\lim _{\epsilon_{0} \rightarrow 0} \frac{\epsilon_{\tau}}{\epsilon_{\tau-1}}=f^{\prime}\left(x_{\tau-1}\right)
$$

Es gilt also:

$$
\lim _{\epsilon_{0} \rightarrow 0} \frac{1}{t} \sum_{\tau=1}^{t} \ln \left|\frac{\epsilon_{\tau}}{\epsilon_{\tau-1}}\right|=\frac{1}{t} \sum_{\tau=1}^{t} \ln \left|f^{\prime}\left(x_{\tau-1}\right)\right| .
$$

Ein weiterer Grenzübergang liefert den exakten Lyapunov-Exponenten

$$
\lambda\left(x_{0}\right)=\lim _{t \rightarrow \infty} \frac{1}{t} \sum_{\tau=1}^{t} \ln \left|f^{\prime}\left(x_{\tau-1}\right)\right| .
$$

Für die logistische Parabel erhält man die Formel

$$
\lambda\left(x_{0}\right)=\lim _{t \rightarrow \infty} \frac{1}{t} \sum_{\tau=1}^{t} \ln \left|4-8 x_{\tau-1}\right|=\ln 4+\lim _{t \rightarrow \infty} \frac{1}{t} \sum_{\tau=0}^{t-1} \ln \left|1-2 x_{\tau}\right| .
$$

Für die (instabilen) Fixpunkte 0 und 1 sowie alle weiteren Anfangswerte, deren Bahnen im Fixpunkt 0 enden, erhält man den Lyapunov-Exponenten

$139 \mathrm{Vgl}$. Peitgen, Jürgens und Saupe (1994), S. 47.

${ }^{140}$ In dieser Arbeit werden numerische Berechnungen von Lyapunov-Exponenten mit einem Mathematica-Programm aus Sandri (1996) durchgeführt.

${ }^{141}$ Mit $\epsilon_{0}$ geht auch $\epsilon_{\tau-1}$ gegen Null. Es muss selbstverständlich die Differenzierbarkeit von $f$ vorausgesetzt werden. Vgl. Peitgen, Jürgens und Saupe (1994), S. 49. 
$\ln 4 \approx 1,39$. Für alle anderen Anfangswerte, also fast sicher ${ }^{142}$, ergibt sich der Lyapunov-Exponent $\ln 2 \approx 0,693$. Daraus ergibt sich auch, dass der Fehler bei Iteration um den Faktor $c=e^{\ln 2}=2$ ansteigt.

Die Interpretation der numerisch ermittelten Lyapunov-Exponenten ist einfach. Der Fehler verändert sich um den Faktor $e^{\lambda\left(x_{0}\right)}$. Ist der LyapunovExponent also positiv, wird der Fehler größer, sonst kleiner. Je größer der Exponent ist, desto ausgeprägter ist die sensitive Abhängigkeit von den Anfangsbedingungen.

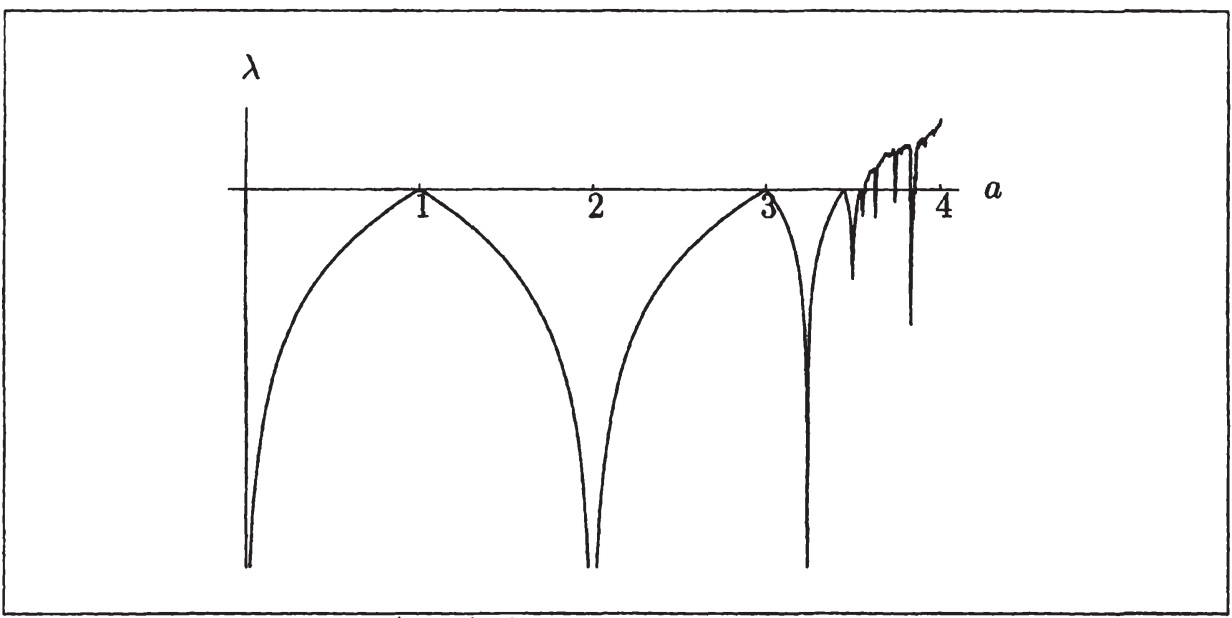

In Anlehnung an: Lorenz (1993), S. 217

Abbildung 3.9: Lyapunov-Exponenten der logistischen Parabel

Abbildung 3.9 zeigt die Lyapunov-Exponenten $\lambda$ der logistischen Parabel $x \mapsto a \cdot x(1-x)$ in Abhängigkeit vom Parameter $a$. Die in Abschnitt 3.6.5 auf analytischem Wege gewonnenen Erkenntnisse lassen sich durch LyapunovExponenten numerisch bestätigen. Für $a<1$ sind die Exponenten negativ, da der Ursprung einen attraktiven Fixpunkt darstellt, gegen den alle Anfangsbahnen konvergieren. Diese Konvergenz wird umso langsamer und damit $\lambda$ umso größer, je näher $a$ der Sattelpunktbifurkation bei $a=1$ kommt. Hier ist $\lambda=0$. Für $1<a<3$ ist der Wert $(a-1) / a$ attraktiver Fixpunkt, so dass die Lyapunov-Exponenten negativ sind. Am superattraktiven Fixpunkt $x=0,5$ für $a=2$ ist $\lambda=-\infty$. Bei der ersten Periodenverdopplung $a=3$ gilt wiederum $\lambda=0$, ebenso bei den weiteren Periodenverdopplungen 3,4495 , 3,5441 usw. Für Werte von $a$ dazwischen konvergiert das System gegen stabile Zyklen mit negativen Lyapunov-Exponenten. Jenseits des Feigenbaum-

${ }_{142}$ Ein Ereignis wird als „fast sicher" bezeichnet, wenn die Wahrscheinlichkeit seines Eintretens eins beträgt. Vgl. Bandelow (1989), S. 196. 
Punktes $a=3,5699 \ldots$ treten dann erstmals positive Lyapunov-Exponenten auf, die auf sensitive Abhängigkeit von den Anfangsbedingungen hinweisen. Es handelt sich nicht um Chaos im Sinne von Definition 3.7, was nur von $a=4$ erfüllt wird, sondern hier erstreckt sich das Chaos nicht auf den gesamten Definitionsbereich, d.h. das Intervall $[0,1]$. Die periodischen Fenster, also diejenigen Parameterwerte jenseits des Feigenbaum-Punktes, an denen das System in stabile Zyklen übergeht, deren Zykluslänge ungleich $2^{n}$ ist, werden durch negative Exponenten angezeigt, am deutlichsten das Fenster der Periode 3 bei $a \approx 3,83$. Für $a=4$ schließlich wird der größte LyapunovExponent $\lambda=\ln 2$ erreicht. ${ }^{143}$

Am Beispiel der logistischen Parabel zeigt sich also, dass Bifurkationen durch $\lambda=0$ angezeigt werden, die Form der Bifurkation aber nicht erkennbar ist. Das Ausmaß der Sensitivität lässt sich ebenfalls ablesen, nicht möglich ist allerdings eine Verifikation der Definition 3.7 von Devaney.

\subsubsection{Numerisches Chaos}

Die topologische Definition von Chaos birgt das Problem, dass sie für ein gegebenes System einen strengen Beweis verlangt, der regelmäßig nur sehr schwierig, wenn überhaupt zu führen ist. Dies gilt in noch größerem Maße für die im nächsten Abschnitt zu behandelnden mehrdimensionalen Systeme.

Glücklicherweise existiert mit dem Lyapunov-Exponenten zumindest ein Maß für sensitive Abhängigkeit, das sich auch auf den mehrdimensionalen Fall und auch bei kontinuierlicher Zeit anwenden lässt. Daher definieren wir:

\section{Definition 3.14}

Ein dynamisches System heißt numerisch chaotisch, wenn es einen beschränkten Zustandsraum und einen positiven Lyapunov-Exponenten besitzt.

Die Forderung nach einem beschränkten Zustandsraum bedeutet, dass der Systemzustand nicht betragsmäßig über alle Grenzen wachsen kann. Dies ist notwendig, weil z.B. auch lineare Systeme einen positiven LyapunovExponenten besitzen. Dennoch kann man hier nicht von chaotischem Verhalten sprechen, weil die relative Abweichung zweier Bahnen voneinander konstant bleibt. ${ }^{144}$ Sensitivität und Chaos können nicht gleichgesetzt werden.

${ }^{143}$ Dieser Sachverhalt wird anhand von Abbildung 3.20 auf S. 87 noch unter einem anderen Aspekt betrachtet werden.

${ }^{144}$ Vgl. Peitgen, Jürgens und Saupe (1994), S. 41f. 
Nun ist der Lyapunov-Exponent in Gleichung (3.15) als ein Grenzwert definiert, dessen Berechnung für ein konkretes System unmöglich sein kann. Doch immerhin lassen sich gute Näherungswerte bestimmen, indem man in Gleichung (3.15) ein hinreichend großes $t$ wählt und die Berechnung von einem Computer durchführen lässt. Dabei muss man sich im Klaren sein, dass eine solche Berechnung keine Beweiskraft in mathematischem Sinne besitzt. Eine Feststellung, die darauf beruht, dass ein System numerisch chaotisch ist, steht nur im Rang einer Hypothese.

\subsection{Chaos in mehrdimensionalen Systemen}

\subsubsection{Grundlagen}

Die bisherige Darstellung hat genügt, das Phänomen des deterministischen Chaos und seine Ursachen zu verdeutlichen. Doch nur in eher seltenen Spezialfällen können ökonomische Sachverhalte in eindimensionalen Modellen abgebildet werden. Die mathematische Darstellung mehrdimensionaler Systeme wurde bereits in den Gleichungen (3.1) und (3.2) eingeführt.

Mehr noch als für eindimensionale Systeme ist die Diskussion um die geeignete Definition von Chaos alles andere als abgeschlossen. ${ }^{145}$ Die bisherigen Definitionen haben den Nachteil, dass der Nachweis auch für recht einfache Systeme nur schwierig zu führen ist.

Glücklicherweise stehen mit dem Konzept des Lyapunov-Spektrums und der fraktalen Dimension seltsamer Attraktoren recht leistungsfähige Hilfsmittel zur Verfügung, zumindest graphische und numerische Hinweise auf Chaos $\mathrm{zu}$ entdecken. Oftmals muss man sich damit zufrieden geben.

\subsubsection{Exkurs 1: Lineare Modelle}

Die Theorie linearer Gleichungssysteme ist sehr weit entwickelt. Zumindest qualitative Ergebnisse lassen sich auch ohne die Hilfe numerischer Simulationen verhältnismäßig leicht gewinnen. Darin ist der Grund zu sehen, warum lineare Modelle in der Ökonomik so weite Verbreitung gefunden haben, obwohl bekannt ist, dass reale wirtschaftliche Zusammenhänge regelmäßig nichtlinearen Charakter haben. Der folgende Exkurs fasst die wichtigsten Eigenschaften

145 Vgl. Kelsey (1988), S. 2. 
linearer Dynamik zusammen, um das Verständnis nichtlinearer Systeme zu erleichtern.

Ein lineares Modell wird beschrieben durch die lineare Abbildung

$$
\mathbf{F}: \mathbb{R}^{n} \rightarrow \mathbb{R}^{n} ; \quad \mathbf{y}=\mathbf{A x} .
$$

Die Matrix A charakterisiert die lineare Abbildung bezüglich der kanonischen Basis des Standardraums. ${ }^{146}$ Lineare Abbildungen haben die Eigenschaften $\mathbf{F}\left(\mathbf{x}_{1}+\mathbf{x}_{2}\right)=\mathbf{F}\left(\mathbf{x}_{1}\right)+\mathbf{F}\left(\mathbf{x}_{2}\right)$ und $\mathbf{F}(c \mathbf{x})=c \mathbf{F}(\mathbf{x})$. Im eindimensionalen Fall reduziert sich das Modell auf die lineare Gleichung $y=a x .^{147}$

Die Analyse dynamischer linearer Modelle erfolgt anhand der Eigenwerte der Matrix A. Ein Eigenwert $\lambda$ mit zugehörigem Eigenvektor $\mathbf{x}$ erfüllt die Gleichung

$$
\mathbf{A x}=\lambda \mathbf{x},
$$

d.h. multipliziert man eine Matrix mit einem Eigenvektor, ist das Ergebnis ein Vielfaches des Eigenvektors, insbesondere ist der Vektor Ax parallel zu x. Man erhält die Eigenwerte als Lösungen des charakteristischen Polynoms ${ }^{148}$

$$
\operatorname{det}(\mathbf{A}-\lambda \mathbf{I})=0 \text {. }
$$

Eine $n \times n$-Matrix hat maximal $n$ verschiedene Eigenwerte, die komplex sein können. Man vereinfacht die Analyse, wenn man sich auf die Jordansche Normalform ${ }^{149}$ beschränkt. Es gilt, dass die Matrizen $\mathbf{A}$ und $\mathbf{S A S}^{-1}$ dieselben Eigenwerte haben wegen

$$
\begin{aligned}
& \operatorname{det}\left(\mathbf{S} \mathbf{A} \mathbf{S}^{-1}-\lambda \mathbf{I}\right)=\operatorname{det}\left(\mathbf{S}(\mathbf{A}-\lambda \mathbf{I}) \mathbf{S}^{-1}\right) \\
& =\operatorname{det}(\mathbf{S}) \operatorname{det}(\mathbf{A}-\lambda \mathbf{I}) \operatorname{det}\left(\mathbf{S}^{-1}\right)=\operatorname{det}(\mathbf{A}-\lambda \mathbf{I}) \text {. }
\end{aligned}
$$

Die Transformation der Matrix A stellt einen Wechsel des Koordinatensystems dar. Die Spaltenvektoren der Matrix S enthalten die Koordinaten der Spaltenvektoren von $\mathbf{A}$ bezüglich des neuen Koordinatensystems. ${ }^{150}$

Wir bemerken, dass hier eine Analogie zu nichtlinearen Systemen besteht. Dort haben wir gesehen, dass sich die dynamischen Eigenschaften eines Systems über eine topologische Konjugation auf ein anderes System übertragen,

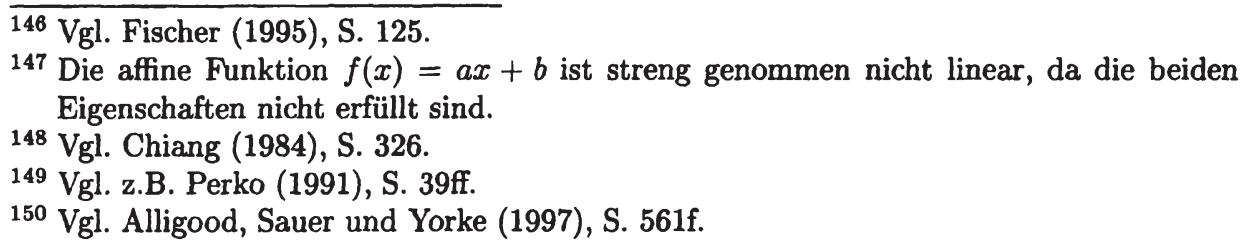


oder anders ausgedrückt: Die dynamischen Eigenschaften topologisch konjugierter Systeme stimmen überein. Hier stellen wir fest, dass lineare Systeme mit ähnlichen Matrizen dieselben Eigenwerte besitzen, die wiederum, wie gleich gezeigt wird, das dynamische Verhalten der linearen Systeme bestimmen. Eine weiterer Zusammenhang zwischen linearen und nichtlinearen Systemen betrifft die das Kriterium für Stabilität. Bei linearen Systemen können die Eigenwerte herangezogen werden, bei nichtlinearen Systemen die Lyapunov-Exponenten. Die Definition der Lyapunov-Exponenten beruht nun auf den Eigenwerten des durch die Jacobi-Matrix linearisierten Systems.

Zur Illustration genügt die Beschränkung auf den $2 \times 2$-Fall. Das charakteristische Polynom hat zwei reelle Lösungen $a$ und $b$, eine reelle Lösung $a$ oder zwei konjugiert-komplexe Lösungen $a \pm i b$. Dazu gehören die drei Normalformen

$$
\mathbf{A}_{1}=\left(\begin{array}{cc}
a & 0 \\
0 & b
\end{array}\right), \quad \mathbf{A}_{2}=\left(\begin{array}{cc}
a & 1 \\
0 & a
\end{array}\right), \quad \mathbf{A}_{3}=\left(\begin{array}{cc}
a & -b \\
b & a
\end{array}\right)
$$

\subsubsection{Zeitdiskrete Modelle}

Ein zeitdiskretes lineares Modell wird beschrieben durch die Gleichung

$$
\mathbf{x}_{t+1}=\mathbf{A} \mathbf{x}_{t}
$$

Der Ursprung ist einziger Fixpunkt des Systems, dessen Stabilität von den Eigenwerten von $\mathbf{A}$ bestimmt wird. Für einen Eigenvektor $\mathbf{x}$ folgt aus Gleichung (3.16)

$$
\mathbf{x}_{t+n}=\mathbf{A}^{n} \mathbf{x}_{t}=\lambda^{n} \mathbf{x}_{t}
$$

Man erkennt, dass ein Eigenvektor für $n \rightarrow \infty$ in den Ursprung strebt genau dann, wenn $|\lambda|<1$. Das lässt sich verallgemeinern für alle Vektoren $\mathbf{x} \in \mathbb{R}^{m}$, wie hier für $m=2$ demonstriert wird. ${ }^{151}$ Dabei können wir uns auf die Normalformen beschränken, da man jede Matrix A durch Koordinatenwechsel auf eine Normalform bringen kann, ohne dass die Transformation die Eigenwerte und damit das dynamische Verhalten des Systems ändern würde.

Für zwei verschiedene reelle Eigenwerte gilt

$$
\left(\begin{array}{l}
x_{n} \\
y_{n}
\end{array}\right)=\left(\begin{array}{ll}
a & 0 \\
0 & b
\end{array}\right)^{n}\left(\begin{array}{l}
x_{0} \\
y_{0}
\end{array}\right)=\left(\begin{array}{cc}
a^{n} & 0 \\
0 & b^{n}
\end{array}\right)\left(\begin{array}{l}
x_{0} \\
y_{0}
\end{array}\right)=\left(\begin{array}{l}
a^{n} x_{n} \\
b^{n} y_{n}
\end{array}\right) .
$$

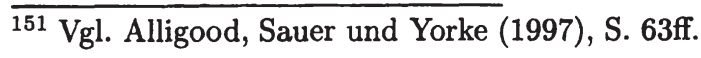


Ein reeller Eigenwert ergibt

$$
\left(\begin{array}{l}
x_{n} \\
y_{n}
\end{array}\right)=\left(\begin{array}{ll}
a & 1 \\
0 & a
\end{array}\right)^{n}\left(\begin{array}{l}
x_{0} \\
y_{0}
\end{array}\right)=a^{n-1}\left(\begin{array}{ll}
a & n \\
0 & a
\end{array}\right)\left(\begin{array}{l}
x_{0} \\
y_{0}
\end{array}\right)=a^{n-1}\left(\begin{array}{c}
a x_{0}+n y_{0} \\
n y_{0}
\end{array}\right) .
$$

Bei zwei konjugiert-komplexen Eigenwerten formt man um zu

$$
\mathbf{A}=\left(\begin{array}{cc}
a & -b \\
b & a
\end{array}\right)=\sqrt{a^{2}+b^{2}}\left(\begin{array}{cc}
\cos \theta & -\sin \theta \\
\sin \theta & \cos \theta
\end{array}\right)
$$

mit $\theta=\arctan (b / a)$. Die komplexen Eigenwerte und Eigenvektoren lassen sich interpretieren als Rotation um den Winkel $\theta$ bei gleichzeitiger Streckung um den Faktor $\sqrt{a^{2}+b^{2}}$.

In allen Fällen gilt

$$
\lim _{n \rightarrow \infty}\left(\begin{array}{l}
x_{n} \\
y_{n}
\end{array}\right)=\left(\begin{array}{l}
0 \\
0
\end{array}\right) \Leftrightarrow \forall k:\left|\lambda_{k}\right|<1
$$

Damit ist die $m=2$-Variante der folgenden Stabilitätsbedingung nachgewiesen:

\section{Satz 3.18}

Der Ursprung ist Attraktor des linearen, zeitdiskreten Systems $\mathbf{x}_{\mathbf{n}}=\mathbf{A x}_{\mathbf{0}}$ genau dann, wenn für alle Eigenwerte $\lambda_{k}$ der Matrix $\mathbf{A}$ gilt:

$$
\left|\lambda_{k}\right|<1 \text {. }
$$

\subsubsection{Zeitstetige Modelle}

Ein zeitstetiges Modell wird dargestellt durch ein System von Differentialgleichungen:

$$
\dot{\mathbf{x}}(t)=\mathbf{A x}(t) .
$$

Der Ursprung ist Gleichgewicht des Systems. Das langfristige Verhalten des Systems hängt wiederum von den Eigenwerten ab. Seien die Anfangsbedingungen $x(0)=x_{0}$ und $y(0)=y_{0}$. Bei zwei verschiedenen reellen Eigenwerten $a$ und $b$ lautet die Lösung

$$
\left(\begin{array}{l}
x(t) \\
y(t)
\end{array}\right)=\left(\begin{array}{l}
x_{0} e^{a t} \\
y_{0} e^{b t}
\end{array}\right)
$$

Das System konvergiert gegen den Ursprung, wenn beide Eigenwerte negativ sind. 
Ein System mit einem reellen Eigenwert $a$ hat die Lösung

$$
\left(\begin{array}{l}
x(t) \\
y(t)
\end{array}\right)=\left(\begin{array}{c}
x_{0} e^{a t}+y_{0} t e^{a t} \\
y_{0} e^{a t}
\end{array}\right)
$$

Für $a<0$ ergibt sich Konvergenz zum Ursprung ${ }^{152}$.

Zwei konjugiert-komplexe Eigenwerte $a \pm i b$ führen zu der Lösung

$$
\left(\begin{array}{l}
x(t) \\
y(t)
\end{array}\right)=\left(\begin{array}{c}
e^{a t}\left(x_{0} \cos b t-y_{0} \sin b t\right) \\
e^{a t}\left(y_{0} \cos b t+x_{0} \sin b t\right)
\end{array}\right) .
$$

Konvergenz zum Ursprung liegt für $a<0$ vor, also wenn der Realteil $\operatorname{Re}\left(\lambda_{k}\right)$ der Eigenwerte negativ ist.

Damit ist die $m=2$-Variante des folgenden Satzes gezeigt:

\section{Satz 3.19}

Der Ursprung ist Attraktor des linearen, zeitstetigen Systems $\dot{\mathbf{x}}(t)=\mathbf{A x}(t)$ genau dann, wenn für alle Eigenwerte $\lambda_{k} \operatorname{der}$ Matrix $A$ gilt:

$$
\operatorname{Re}\left(\lambda_{k}\right)<0 .
$$

\subsubsection{Linearisierung}

Trotz der nichtlinearen Natur ökonomischer Wirkungszusammenhänge beschränkt man sich in Modellen häufig auf lineare Näherungen. In der Umgebung eines Gleichgewichtspunktes ist diese Näherung auch gerechtfertigt.

Sei $\mathbf{f}: \mathbb{R}^{n} \rightarrow \mathbb{R}^{n}$ eine differenzierbare Abbildung und $\mathbf{x}, \mathbf{h} \in \mathbb{R}^{n}$. Dann gilt

$$
\mathbf{f}(\mathbf{x}+\mathbf{h})-\mathbf{f}(\mathbf{x}) \approx \operatorname{Df}(\mathbf{x}) \mathbf{h} .
$$

Dies ist ein lineares System, bei dem die Jacobi-Matrix von $\mathbf{f}$ die Rolle der Matrix A übernimmt. In der Nähe des Punktes $\mathbf{x}$ ist die Approximation hinreichend genau, so dass sie für eine Prüfung der lokalen Stabilität von $\mathbf{x}$ brauchbar ist.

Dieser Exkurs hat gezeigt, dass das dynamische Verhalten eines linearen Systems allein aus seinen Eigenwerten bestimmt werden kann - bei zeitdiskreten Systemen aus den Beträgen, bei zeitstetigen Systemen aus den Realteilen der Eigenwerte. Diese Ergebnisse gelten auch lokal für nichtlineare Systeme, indem man dort auf eine Linearisierung mittels Jacobi-Matrix zurückgreift.

$\overline{152}$ Man beachte, dass für $a<0$ gilt: $\lim _{t \rightarrow 0} t e^{a t}=0$. Vgl. z.B. Forster (1993), S. 76. 
Abschließend sei darauf hingewiesen, dass die Bestimmung von Eigenwerten vor allem in höherdimensionalen Systemen überaus mühsam sein kann, da das charakteristische Polynom eines $n$-dimensionalen Systems $n$-ten Grades ist. Daher sind eine Reihe von Kriterien entwickelt worden, die zumindest die Klärung der Frage erlauben, ob die Eigenwerte die für Stabilität notwendigen Größen aufweisen. Die umfassendsten Kriterien sind das Routh-HurwitzTheorem ${ }^{153}$ für Differentialgleichungssysteme und das Schur-Theorem ${ }^{154}$ für Differenzengleichungssysteme.

\subsubsection{Exkurs 2: Zeitstetige Modelle der Dimensionen eins und zwei}

In diesem Exkurs wird gezeigt, warum eine kontinuierliche Modellierung bei einer und auch bei zwei Modellvariablen kein chaotisches Verhalten erzeugen kann. Betrachten wir zunächst als eindimensionales Beispiel die VerhulstDynamik in Form einer Differenzengleichung

$$
x_{t+1}=x_{t}+a \cdot x_{t}\left(1-x_{t}\right)
$$

im Vergleich mit derselben Dynamik, nun aber als Differentialgleichung ${ }^{155}$

$$
\dot{x}(t)=a \cdot x(t)(1-x(t)) .
$$

Den Zusammenhang zwischen den beiden Gleichungen erhält man durch Grenzübergang der Periodenlänge $\Delta_{t} \rightarrow 0$.

Das Langzeitverhalten der Verhulst-Differentialgleichung wird erkennbar, wenn man die Trajektorie für die Anfangsbedingung $x(0)=x_{0}$ berechnet:

$$
x(t)=\frac{e^{a \cdot t}}{e^{a \cdot t}-1+\frac{1}{x_{0}}} \text { mit } \lim _{t \rightarrow \infty} x(t)=1 \text { für alle } x_{0}>0 .
$$

Alle Trajektorien konvergieren gegen den Gleichgewichtswert eins.

Die Abbildung 3.10 zeigt am Beispiel der Verhulst-Dynamiken (3.17) und (3.18) den entscheidenden Unterschied, auf dem die Unmöglichkeit chaotischen Verhaltens einer Differentialgleichung beruht. Die Trajektorien einer Differentialgleichung können sich im Gegensatz zu den Bahnen einer Differenzengleichung niemals schneiden. Das Gegenargument macht dies von einer

153 Vgl. Chiang (1984), S. 546f.

154 Vgl. Chiang (1984), S. 601f.

155 Vgl. Peitgen, Jürgens und Saupe (1994), S. $240 \mathrm{ff}$. 

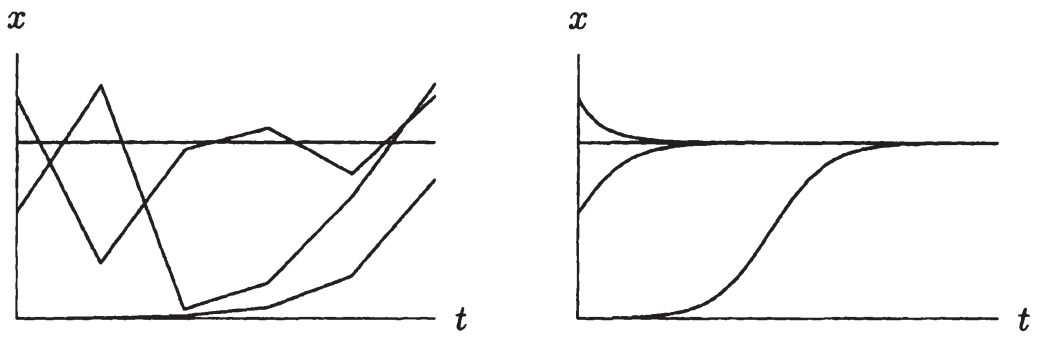

In Anlehnung an: Peitgen, Jürgens und Saupe (1994), S. 242

Abbildung 3.10: Diskrete und stetige Verhulst-Dynamik

anderen Seite verständlich. Haben zwei Trajektorien auch nur einen Punkt gemeinsam, müssen sie für alle Zeiten aufeinander verlaufen. Dasselbe gilt für Bahnen an Stellen $t \in \mathbb{Z}$, während Schnittpunkte für $t \notin \mathbb{Z}$ für Bahnen mit verschiedenen Startwerten möglich sind.

Der folgende Satz bildet das theoretische Fundament des Beispiels ${ }^{156}$ :

\section{Satz 3.20}

Sei $E$ ein normierter Vektorraum, $W \subset E$ eine offene Teilmenge, $\mathbf{f}: W \rightarrow E$ eine stetig differenzierbare Abbildung und $\mathbf{x}_{0} \in W$. Dann gibt es ein $a \in \mathbb{R}^{+}$ und eine eindeutige Lösung $\mathbf{x}:(-a, a) \rightarrow W$ der Differentialgleichung $\dot{\mathbf{x}}=$ $\mathbf{f}(\mathbf{x})$, die der Anfangsbedingung $\mathbf{x}(0)=\mathbf{x}_{0}$ genügt.

Aus dem Satz kann man folgern, dass sich weder Trajektorien verschiedener Anfangswerte noch eine Trajektorie mit sich selbst schneiden können. ${ }^{157}$ Oder anders ausgedrückt: „Durch jeden Punkt der $x y$-Ebene geht genau eine Trajektorie, und diese besitzt eine wohlbestimmte Richtung." ${ }^{158}$

Diese Tatsache hat zur Folge, dass das Verhalten zweidimensionaler Systeme ebenfalls in wenigen Typen kategorisiert werden kann. Gegenüber eindimensionalen Systemen, die nur Fixpunkte als Attraktoren kennen ${ }^{159}$, sind nun auch geschlossene Kurven in der $x y$-Ebene als Attraktoren möglich. Dass

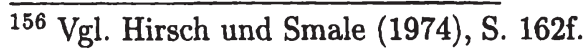

157 Vgl. Hirsch und Smale (1974), S. 167f. und Heuser (1991), S. 534.

${ }^{158}$ Heuser (1991), S. 534f.

159 Auch $\pm \infty$ sind mögliche Attraktoren. Die Lösung einer solchen Differentialgleichung heißt divergent. Außerdem sind periodisch schwankende Zeitpfade möglich. Vgl. Chiang (1984), S. 493ff. 
es damit in der Tat sein Bewenden hat, bestätigt das Theorem von Poincaré und Bendixson. ${ }^{160}$

\section{Satz 3.21}

Eine nichtleere, kompakte Grenzmenge eines stetig differenzierbaren zweidimensionalen Differentialgleichungssystems, die keinen Fixpunkt enthält, ist eine geschlossene Trajektorie.

Chaotisches Verhalten ist damit für ein zweidimensionales Differentialgleichungssystem unmöglich. ${ }^{161}$
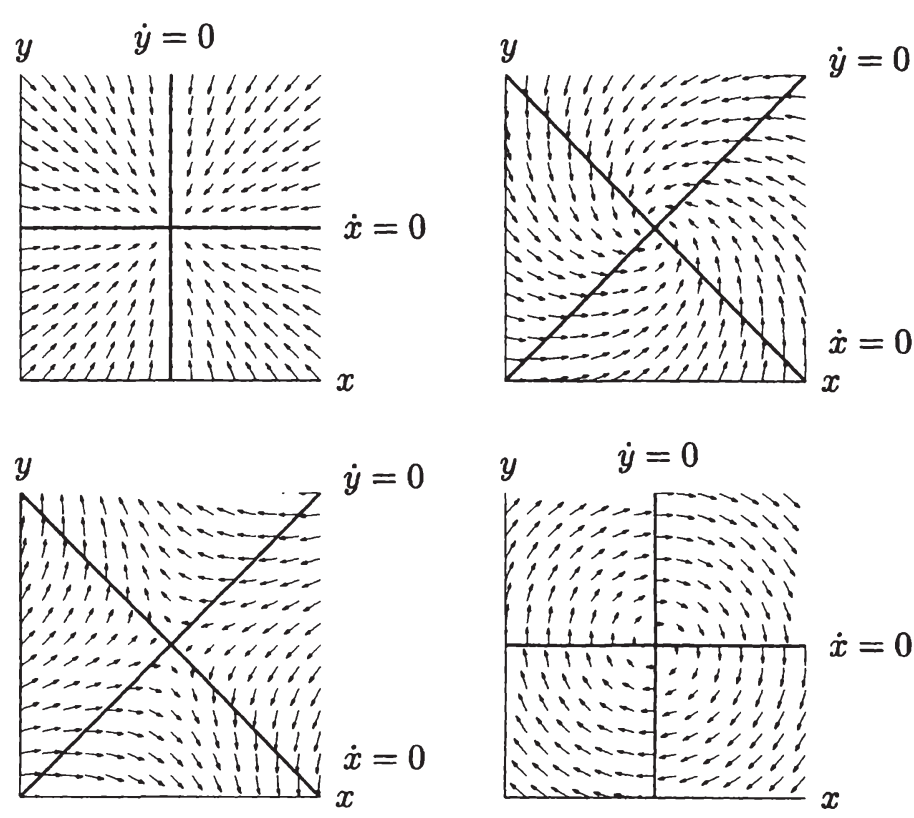

In Anlehnung an: Chiang (1984), S. 634

Abbildung 3.11: Phasendiagramme zweidimensionaler Differentialgleichungssysteme

Abbildung 3.11 zeigt die drei möglichen Gleichgewichte und unten rechts eine geschlossene Trajektorie. Die Schnittpunkte der beiden Loki $\dot{x}=0$ und $\dot{y}=0$ stellen Gleichgewichte dar, in denen sich $x$ und $y$ nicht mehr verändern. Sie können entweder direkt erreicht werden (Knoten) oder in spiralförmiger

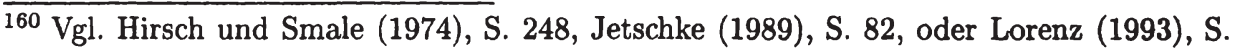
41.

$161 \mathrm{Vgl}$. Kelsey (1988), S. 11. 
Annäherung (Fokus). Beide Formen existieren auch in instabiler Form, wobei die Vektorfelder von den Gleichgewichten weg weisen. Eine Zwischenform ist der Sattelpunkt, der je nach Ausgangspunkt der Trajektorie anziehend oder abstoßend ist.

Zusätzlich zu den dargestellten Fällen sind auch multiple Gleichgewichte möglich, wenn die Gleichgewichtsloki nicht monoton sind und mehrere Schnittpunkte haben. Doch auch dann bestehen die Grenzmengen nur aus geschlossenen Trajektorien, Gleichgewichten und Trajektorien, die Gleichgewichte verbinden. ${ }^{162}$

Als Ergebnis dieses Exkurses lässt sich festhalten, dass in Differentialgleichungssystemen erst ab drei Gleichungen nach chaotischen Trajektorien gesucht zu werden braucht. Anschaulich ausgedrückt fehlt Trajektorien in der Ebene oder gar auf einer Geraden der Platz für nichtkonvergentes Verhalten. Erst im Raum können sie sich beliebig umeinander winden und zu seltsamen Attraktoren formen.

\subsubsection{Definitionen}

Die Versuche, chaotisches Verhalten mehrdimensionaler Systeme in eine exakte Definition zu fassen, sind recht zahlreich und unterscheiden sich graduell oder auch erheblich voneinander. Gemein ist ihnen lediglich, dass keine als allgemein anerkannt gelten kann. Insofern sind bereits die Grundlagen als weitgehend ungeklärt anzusehen. Hinzu kommen die erheblichen Schwierigkeiten, die die Analyse nichtlinearer mehrdimensionaler Systeme bereitet. Nicht ohne Grund werden solche Systeme in der Regel linearisiert mit der Folge, dass die gefundenen Ergebnisse zwar eindeutig sind, aber nur lokal um den Punkt der Linearisierung gelten.

Die Definition 3.7 von Devaney lässt sich auf höherdimensionale diskrete Systeme übertragen und ebenso auf Systeme, die nicht im Euklidischen Raum definiert sind, d.h. Systeme auf Kreisen, Tori oder Cantor-Mengen. ${ }^{163}$ Man muss sich lediglich klarmachen, dass in Definition 3.7 zur Messung von Abständen die Betragsfunktion $d(x, y)=|x-y|$ verwendet wird, die die für $\mathbb{R}$ natürliche Metrik darstellt. Für Systeme im $\mathbb{R}^{n}$ wäre dann beispielsweise die Euklidische Norm $d(\mathbf{x}, \mathbf{y})=\|\mathbf{x}-\mathbf{y}\|=\sqrt{\sum\left(x_{i}^{2}-y_{i}^{2}\right)}$ zu verwenden. Das Problem dieser Definition ist, dass die geforderten Eigenschaften nur in

162 Vgl. Lorenz (1993), S. 39, sowie Guckenheimer und Holmes (1983), S. $44 f$.

163 Vgl. Devaney (1989), S. 50 und 205. 
äußerst seltenen Fällen direkt nachweisbar sind. Eine Erleichterung stellt wiederum die topologische Konjugation ${ }^{164}$ dar, mit deren Hilfe von der Chaoseigenschaft eines Systems auf die eines dazu konjugierten Systems geschlossen werden kann. ${ }^{165}$

Eine frühere Definition gibt Marotto. ${ }^{166}$ Sie stellt eine Erweiterung des Theorems von Li und Yorke dar. ${ }^{167}$ Dieses Theorem war ein beliebtes, weil leicht zu prüfendes Kriterium. Ein System, das einen dreiperiodigen Zyklus besitzt, weist Eigenschaften auf, die man als Chaos bezeichnen kann. Allerdings ist es möglich, dass die Menge der in diesem Sinne chaotischen Bahnen das Lebesgue-Maß Null hat, also faktisch nicht beobachtbar ist. Das Theorem von Marotto setzt Chaos mit der Existenz eines sogenannten homoklinen Punktes gleich. Solche Systeme weisen ebenfalls Eigenschaften wie Sensitivität auf. Problematisch ist jedoch das Entdecken homokliner Punkte. In der Regel müssen umfangreiche Simulationsrechnungen durchgeführt werden, so dass der Nachweis chaotischen Verhaltens einen eher experimentellen Charakter erhält. ${ }^{168}$ Der Nachteile der Definition nach Li und Yorke bleibt darüber hinaus bestehen.

Chaos in mehrdimensionalen Systemen wird oft mit der Existenz eines seltsamen Attraktors gleichgesetzt. Doch auch für den seltsamen Attraktor gibt es keine allgemein anerkannte Definition. In Definition 3.5 wurde bereits der Begriff des Attraktors eingeführt. Wie nun als Spezialfall ein seltsamer Attraktor zu charakterisieren ist, ist von Quelle zu Quelle verschieden.

Peitgen, Jürgens und Saupe unterscheiden die Attribute seltsam und chaotisch. Seltsam ist ein Attraktor, wenn er eine fraktale Struktur hat; chaotisch ist er, wenn er sensitive Abhängigkeit von den Anfangsbedingungen aufweist. ${ }^{169}$ Jetschke hingegen setzt seltsam mit Sensitivität gleich. ${ }^{170}$ Ohne hier auf Detailprobleme der Definitionen einzugehen, sei gesagt, dass man bei der Prüfung der sensitiven Abhängigkeit und der fraktalen Struktur in der Regel auf numerische Hilfsmittel angewiesen ist. Lyapunov-Exponenten und fraktale Dimensionsmaße spielen hier eine große Rolle und werden im Folgenden vorgestellt.

\footnotetext{
164 Vgl. Definition 3.12 auf Seite 47.

165 Für ein Beispiel vgl. Devaney (1989), S. 208.

166 Vgl. Marotto (1978).

$167 \mathrm{Vgl}$. Li und Yorke (1975). Auf den noch früheren Definitionsversuch in Diamond (1976) wird hier nicht eingegangen.

${ }^{168}$ Vgl. Loistl und Betz (1996), S. 47.

169 Vgl. Peitgen, Jürgens und Saupe (1994) S. 232.

170 Vgl. Jetschke (1989), S. 143.
} 
Abschließend sei noch präzisiert: ${ }^{171}$

\section{Definition 3.15}

Ein System von Differenzengleichungen $\mathbf{x}_{t+1}=\mathbf{f}\left(\mathbf{x}_{t}\right)$ bzw. Differentialgleichungen $\dot{\mathbf{x}}=\mathbf{f}(\mathbf{x})$ mit $\mathbf{x} \in \mathbb{R}^{n}$ heißt chaotisch, wenn es einen Attraktor mit folgenden Eigenschaften besitzt: Das Lyapunov-Spektrum enthält ein positives Element und die Lyapunov-Dimension ist keine ganze Zahl. Ein solcher Attraktor heißt seltsamer Attraktor.

Die Begriffe Lyapunov-Spektrum und Lyapunov-Dimension beschreiben numerische Maße für Sensitivität und fraktale Struktur und werden in den folgenden Abschnitten eingeführt. Da sie aufeinander aufbauen, ist für einen gegebenen Attraktor in der Regel entweder keine oder es sind beide Eigenschaften erfüllt.

\subsubsection{Lyapunov-Spektrum}

Der Lyapunov-Exponent für eindimensionale Systeme wurde bereits in Abschnitt 3.4.2 vorgestellt. Er ist ein Maß dafür, wie sich zwei Bahnen mit infinitesimal kleiner Differenz im Anfangswert zueinander verhalten, mithin ein Maß für die sensitive Abhängigkeit von den Anfangsbedingungen. Ein positiver Exponent $\lambda$ bedeutet, dass sich die Bahnen mit jedem Iterationsschritt im Durchschnitt um den Faktor $e^{\lambda}$ voneinander entfernen.

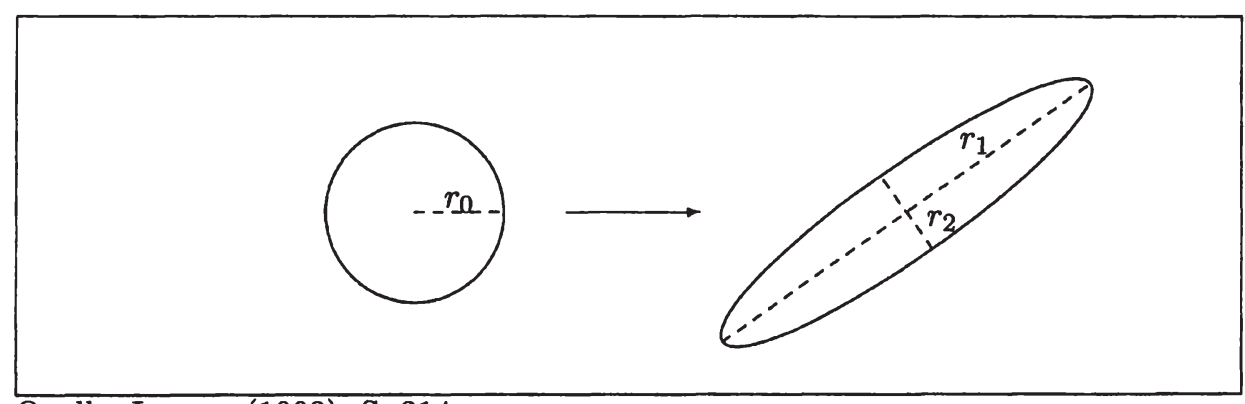

Quelle: Lorenz (1993), S. 214.

Abbildung 3.12: Verformung einer Kreisscheibe

Lyapunov-Exponenten sind auch für mehrdimensionale stetige und diskrete Systeme definiert. Nur muss hier das Auseinanderlaufen der Bahnen in 171 Vgl. Lorenz (1993), S. 170. 
$n$ Dimensionen betrachtet werden; dementsprechend gibt es $n$ LyapunovExponenten. Man bezeichnet die Menge der Lyapunov-Exponenten als Lyapunov-Spektrum. ${ }^{172}$ So zeigt Abbildung 3.12, wie eine Kreisscheibe durch Iteration zu einer Ellipse verformt wird. Die Richtungen, in der die Dehnungen bzw. Stauchungen gemessen werden, stehen senkrecht aufeinander, und die Exponenten werden üblicherweise der Größe nach geordnet. ${ }^{173}$ Der Abbildung ist auch zu entnehmen, dass die Richtungen nicht mit den Basisvektoren des Euklidischen Raumes übereinstimmen und sich durch Iteration drehen können.

Weist ein dynamisches System einen positiven Lyapunov-Exponenten auf, ist es sensitiv abhängig von den Anfangsbedingungen. Beliebig nah beieinander liegende Bahnen divergieren im Zeitablauf. Dies ist schon aus der Diskussion eindimensionaler Systeme bekannt. Doch auch die Summen von Lyapunov-Exponenten lassen sich interpretieren. Die Summe der ersten $k$ Exponenten ist der maximale durchschnittliche Faktor, um den sich ein $k$ dimensionales Volumenelement verändert. ${ }^{174}$

Der Ellipsoid in Abbildung 3.12 entsteht durch Multiplikation der Kreisscheibe mit einer quadratischen Matrix A. Die Länge der Halbachsen der Ellipse wird durch Singulärwertzerlegung von $\mathbf{A}$ bestimmt. Dazu benötigt man die Eigenwertzerlegungen der symmetrischen Matrizen $\mathbf{A A}^{\prime}=\mathbf{U} \mathbf{\Lambda} \mathbf{U}^{\prime}$ und $\mathbf{A}^{\prime} \mathbf{A}=\mathbf{V} \boldsymbol{\Lambda} \mathbf{V}^{\prime} . \boldsymbol{\Lambda}$ ist eine Diagonalmatrix, die die Eigenwerte $\lambda_{\boldsymbol{i}}$ von $\mathbf{A A}^{\prime}$ bzw. AA ${ }^{\prime}$ enthält ${ }^{175}$, wobei $\forall i: \lambda_{i} \in \mathbb{R}_{0}^{+}$. U bzw. $\mathbf{V}$ enthalten die Eigenvektoren von $\mathbf{A A}^{\prime}$ bzw. $\mathbf{A A}^{\prime}$ und sind orthonormal ${ }^{176}$. Sei außerdem $\mathbf{S}$ eine Diagonalmatrix mit den Einträgen $s_{i}=\sqrt{\lambda_{i}}$. Dann heißen die $s_{i}$ Singulärwerte und $\mathbf{A}=\mathbf{U S V}^{\prime}$ Singulärwertzerlegung von $\mathbf{A}$.

Wegen $\left\|\mathbf{v}_{i}\right\|=1$ liegt $\mathbf{v}_{i}$ auf der $n$-dimensionalen Einheitskugel, und es gilt $\mathbf{A} \mathbf{v}_{i}=s_{i} \mathbf{u}_{i}$. Durch die Multiplikation mit $\mathbf{A}$ entsteht also aus einem System orthonormaler Vektoren ein System orthogonaler Vektoren der Längen $s_{i}$. Jeder Vektor $\mathbf{x}$ lässt sich als Linearkombination $\mathbf{x}=\sum_{i} a_{i} \mathbf{v}_{i}$ darstellen mit $\|\mathbf{x}\|=\sum_{i} a_{i}$. Damit gilt $\mathbf{A x}=\sum_{i} a_{i} \mathbf{A} \mathbf{v}_{i}=\sum_{i} a_{i} s_{i} \mathbf{u}_{i}$ und $\|\mathbf{A x}\|=\sum_{i} a_{i} s_{i}$. Jeder Vektor $\mathbf{x}$ der Länge eins wird also auf einen Ellipsoid mit Halbachsenlängen $s_{i}$ abgebildet.

Wir untersuchen nun, wie sich Flüsse verhalten, die in der $\epsilon$-Umgebung $U_{\epsilon}$ eines Ausgangspunkts $\mathrm{x}_{0}$ liegen. Eine Abbildung $\mathrm{f}$ formt aus $U_{\epsilon}$ den Ellipsoi-

172 Vgl. Wolf (1986), S. 277.

173 Vgl. Alligood, Sauer und Yorke (1997), S. 194.

174 Vgl. Peitgen, Jürgens und Saupe (1994), S. 283.

${ }^{175} \mathrm{Da}$ die beiden Matrixprodukte symmetrisch zueinander sind, stimmen ihre Eigenwerte überein.

${ }^{176}$ Dies folgt wiederum aus der Symmetrie der Matrixprodukte. 
den $\mathbf{D f}\left(\mathbf{x}_{0}\right) U_{\epsilon}$ bzw. nach $n$ Iterationsschritten $\mathbf{D f}^{n}\left(\mathbf{x}_{0}\right) U_{\boldsymbol{\epsilon}}$. Die Verwendung der Jacobi-Matrix als lineare Approximation ist deswegen statthaft, weil infinitesimal kleine Abweichungen von $\mathbf{x}_{0}$ untersucht werden sollen. ${ }^{177}$ Wie oben dargestellt, werden die Halbachsen des Ellipsoiden $\mathbf{D} \mathbf{f}^{n}\left(\mathbf{x}_{0}\right) U_{\epsilon}$ von den Singulärwerten der Jacobi-Matrix bestimmt, die sich als Quadratwurzeln der Eigenwerte der Matrix $\mathbf{D f}^{n}\left(\mathbf{x}_{0}\right)\left(\mathbf{D f}^{n}\left(\mathbf{x}_{0}\right)\right)^{\prime}$ berechnen lassen.

Zur Folge der Iterationen gehört eine Folge von Singulärwertspektren, die die Expansion oder Kontraktion entlang der Halbachsen beschreibt. Üblicherweise ordnet man die Singulärwerte der Größe nach. Das durchschnittliche Expansionsverhalten pro Iterationsschritt berechnet man als geometrisches Mittel der Singulärwerte; der natürliche Logarithmus des Mittels ist der Lyapunov-Exponent. ${ }^{178}$

\section{Definition 3.16}

Sei $\mathbf{f}: \mathbb{R}^{m} \rightarrow \mathbb{R}^{m}$ eine stetig differenzierbare Abbildung, und für $k=1, \ldots, m$ sei $s_{k}^{n}$ der $k$-größte Singulärwert der Matrix $\operatorname{Df}^{n}\left(\mathbf{x}_{0}\right)$. Dann heißt

$$
\lambda_{k}=\ln \lim _{n \rightarrow \infty}\left(s_{k}^{n}\right)^{1 / n}
$$

der $k$-te Lyapunov-Exponent von $\mathbf{f}$.

Die Ermittlung der Abbildung $\mathbf{f}^{n}$ ist nicht notwendig. Statt dessen folgt aus der Kettenregel

$$
\operatorname{Df}^{n}\left(\mathbf{x}_{0}\right)=\prod_{i=0}^{n-1} \operatorname{Df}\left(\mathbf{x}_{i}\right)
$$

Das Problem liegt eher darin, dass nach einigen Iterationen die Singulärwerte sehr groß bzw. klein werden. Rundungsfehler bei computergestützter Berechnung führen zu verfälschten Ergebnissen. Man umgeht dieses Problem durch ein Orthonormalisierungsverfahren, das hier kurz vorgestellt wird. ${ }^{179}$

Wir beginnen mit einer orthonormalen Basis $\left\{\mathbf{w}_{1}^{0}, \ldots \mathbf{w}_{m}^{0}\right\}$ für $\mathbb{R}^{m}$ und berechnen

$$
\forall i=1, \ldots, m: \mathbf{z}_{i}=\mathbf{D f}\left(\mathbf{w}_{i}^{0}\right) .
$$

Die Vektoren $\mathbf{z}_{i}$ liegen auf dem Ellipsoiden $\mathbf{D f}\left(\mathbf{x}_{0}\right) U$, sind aber im Allgemeinen nicht orthogonal. Mit dem Orthogonalisierungsverfahren von GramSchmidt wird eine Menge von orthogonalen Vektoren $\left\{\mathbf{w}_{1}^{1}, \ldots \mathbf{w}_{m}^{1}\right\}$ generiert,

177 Siehe hierzu auch die Diskussion des Lyapunov-Exponenten eines eindimensionalen Systems, insbesondere Gleichung (3.15) auf Seite 59.

178 Vgl. Alligood, Sauer und Yorke (1997), S. 195.

179 Vgl. Alligood, Sauer und Yorke (1997), S. 200. 
die einen Ellipsoiden gleichen Volumens definiert. Führt man dieses Verfahren hinreichend oft durch, erhält man aus den Vektoren $\mathbf{w}_{i}^{n}$ Näherungswerte für das durchschnittliche Wachstum in die verschiedenen Richtungen. Um das Problem der Rundungsfehler zu lösen, werden die orthogonalen Vektoren vor jedem Schritt normalisiert.

Zur numerischen Berechnung von Lyapunov-Exponenten ist eine ganze Reihe von Verfahren entwickelt worden. ${ }^{180}$ Manche dieser Verfahren sind sogar für experimentelle Daten geeignet, bei denen das zugrunde liegende Gleichungssystem nicht bekannt ist.

\subsubsection{Fraktale Dimension}

Nach unserer Anschauung hat der uns umgebende Raum drei Dimensionen, eine Ebene zwei. Eine Linie identifizieren wir mit der Dimension eins, und Physiker pflegen in den vier Dimensionen der Raumzeit zu denken. Die Dimension kann man dabei denken als die Anzahl der unabhängigen Koordinaten, die notwendig ist, um einen Punkt zu lokalisieren. ${ }^{181}$ Lässt man die Anschauung, die ja schon bei der vierdimensionalen Raumzeit einige Schwierigkeiten bereitet, beiseite, ergibt sich die Verallgemeinerung auf den $n$-dimensionalen Raum beinahe von selbst.

Bei der Entwicklung eines mathematisch präzisen Dimensionsbegriffs kam es vor allem darauf an, dass topologisch äquivalente Figuren auch dieselbe Dimension haben. Topologische Äquivalenz bedeutet, dass man die Figuren durch Stauchung, Dehnung oder Krümmung ineinander überführen kann, als bestünde der sie umgebende Raum aus Gummi. ${ }^{182}$ Dies entspricht etwa dem Effekt, den die Gravitation auf die Raumzeit hat, was sich z.B. in der Ablenkung von Lichtstrahlen zeigt. ${ }^{183}$ Schnittpunkte von Linien oder Löcher in Figuren, z.B. in einem Torus, bleiben hingegen unverändert. Es entspricht der Intuition, dass solchermaßen ähnliche Objekte auch dieselbe Dimension haben sollten.

Die Überdeckungsdimension ist in diesem Sinne topologisch invariant. Sie wird berechnet, indem man die zu betrachtende Figur mit offenen Kugeln so

180 Vgl. Shimada und Nagashima (1979), Bennettin, Galgani, Giorgilli und Strelcyn (1980), Wolf (1986), Eckmann und Ruelle (1985), Kurths und Herzel (1987). Eine vergleichende Diskussion findet sich bei Geist, Parlitz und Lauterborn (1990) und Loistl und Betz (1996).

181 Vgl. Peitgen, Jürgens und Saupe (1992), S. 130.

182 Vgl. Peitgen, Jürgens und Saupe (1992), S. 128.

${ }^{183}$ Vgl. Gerthsen (1997), S. 861. 


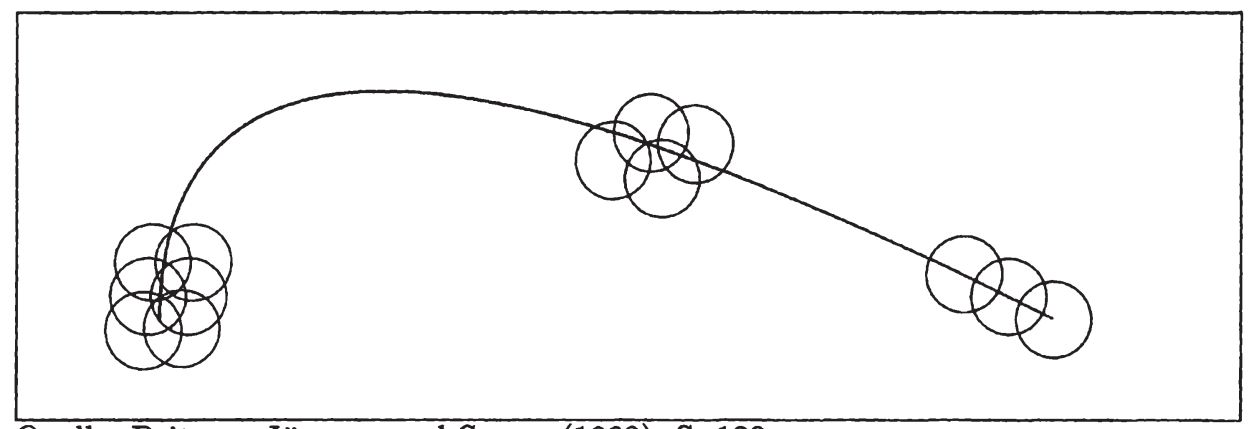

Quelle: Peitgen, Jürgens und Saupe (1992), S. 133

Abbildung 3.13: Überdeckung einer Kurve durch Kreise

überdeckt, dass sie sich möglichst wenig überschneiden. Gelingt eine Überdeckung dergestalt, dass alle Schnittmengen zwischen den Kugeln leer sind, hat die Figur die Dimension null. Dies ist der Fall bei unzusammenhängenden Punktmengen. Bei einer Kurve ist es möglich, diese mit Kreisscheiben, also Kugeln der Dimension zwei, zu überdecken, so dass sich immer nur je zwei Kreise überschneiden. Dies ist in Abbildung 3.13 dargestellt. Dort sieht man auch, dass durchaus Überdeckungen möglich sind, bei denen sich jeweils mehr als zwei Kreise überschneiden, jedoch keine Überdeckung ganz ohne Überschneidung. Um sich dies zu verdeutlichen, bemerke man, dass die Kreise offen sind, die Ränder also nicht dazugehören.

Die Überdeckungsdimension ist immer eine natürliche Zahl. Die Entdeckung raumfüllender Kurven hat aber gezeigt, dass diese intuitiv geprägte Vorstellung von Dimension nicht genügt. Peano und Hilbert diskutierten Kurven, die jeden Punkt eines Flächenstücks treffen. ${ }^{184} \mathrm{Zwar}$ gelang der Nachweis, dass die Vorstellung von der topologischen Invarianz richtig ist, doch wurden im Zuge dieser Diskussion auch Dimensionsbegriffe entwickelt, die auf andere Eigenschaften der Figuren abzielen. Diese Dimensionen können auch gebrochene Zahlen sein, was den Objekten mit nicht-ganzzahliger Dimension den Namen Fraktale eingetragen hat. ${ }^{185}$

Ein einfach zu konstruierendes Fraktal ist die Koch-Kurve in Abbildung 3.14. ${ }^{186}$ Die Idee ist, eine stetige Kurve zu finden, die an keinem Punkt differenzierbar ist. Sie müsste also aus lauter Ecken bestehen, so dass sich nirgends

$\overline{184}$ Vgl. Peano (1890) und Hilbert (1891), zitiert nach Peitgen, Jürgens und Saupe (1992), S. 115.

${ }^{185}$ Der Begriff ist abgeleitet vom lateinischen frangere - zerbrechen.

186 Vgl. von Koch (1904) und von Koch (1906), zitiert nach Peitgen, Jürgens und Saupe (1992), S. 107. 


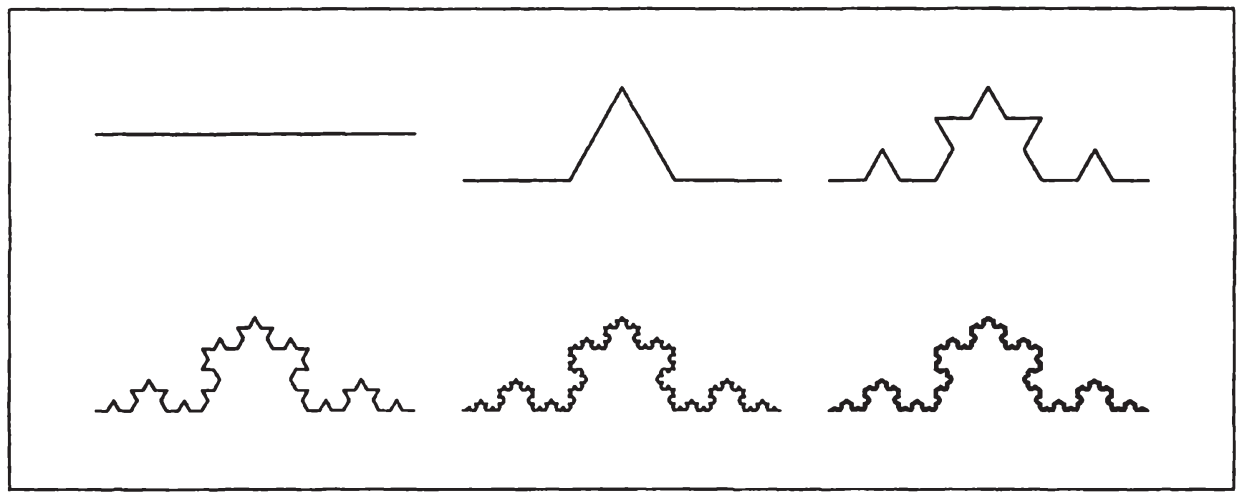

In Anlehnung an: Peitgen, Jürgens und Saupe (1992), S. 112

\section{Abbildung 3.14: Konstruktion der Koch-Kurve}

eine Tangente anlegen lässt. Ausgangspunkt der Konstruktion ist eine Strecke, deren mittleres Drittel durch ein gleichseitiges Dreieck ohne Grundseite ersetzt wird. Für die entstehenden vier Strecken wird diese Prozedur wiederholt. Setzt man fort bis ins Unendliche, erhält man als Grenzobjekt die Koch-Kurve. Eine andere Vorgehensweise führt zum selben Ergebnis, lässt sich aber leichter exakt beschreiben. Man verkleinere die Ausgangsfigur um den Faktor 1/3 und klebe vier Kopien davon so zusammen, dass die oben beschriebene Figur entsteht. Bezeichnet man die vier Ähnlichkeitstransformationen (Skalierung und Anordnung) mit $w_{i}, \quad i=1, \ldots, 4$, ist das Bild die Vereinigungsmenge $W$ der transformierten Kopien der Ausgangsfigur $A$ :

$$
W(A)=w_{1}(A) \cup w_{2}(A) \cup w_{3}(A) \cup w_{4}(A)
$$

Die Transformation $W$ wird auch Hutchinson-Operator genannt. ${ }^{187}$ Die $w_{i}$ lassen sich durch folgende Gleichungen beschreiben ${ }^{188}$

$$
\begin{aligned}
& w_{1}(x, y)=\left(\frac{1}{3} x, \frac{1}{3} y\right) \\
& w_{2}(x, y)=\left(\frac{1}{6} x-\frac{\sqrt{3}}{6} y+\frac{1}{3}, \frac{\sqrt{3}}{6} x+\frac{1}{6} y\right) \\
& w_{3}(x, y)=\left(\frac{1}{6} x+\frac{\sqrt{3}}{6} y+\frac{1}{2},-\frac{\sqrt{3}}{6} x+\frac{1}{6} y+\frac{\sqrt{3}}{6}\right) \\
& w_{4}(x, y)=\left(\frac{1}{3} x+\frac{1}{2}, \frac{1}{3} y\right)
\end{aligned}
$$

${ }_{187}$ Vgl. Hutchinson (1981).

188 Vgl. Peitgen, Jürgens und Saupe (1992), S. 202. 
Bezeichnet man die Koch-Kurve mit $K$, lässt sie sich als Fixpunkt des Hutchinson-Operators $W$ darstellen ${ }^{189}$ :

$$
W(K)=K
$$

In dieser Formulierung lässt sich ein Phänomen erkennen, das für Fraktale charakteristisch ist, die Selbstähnlichkeit. Da der Operator $W$ die Koch-Kurve unverändert lässt, muss eine Vergrößerung eines Teils der Koch-Kurve um den Faktor drei wieder die Koch-Kurve ergeben, oder anders ausgedrückt: Das Detail gleicht dem Ganzen exakt.

Die Koch-Kurve ist topologisch äquivalent zu einer Strecke, was sich auch in der gleichen Überdeckungsdimension eins ausdrückt. In einer anderen Hinsicht unterscheiden sich die beiden Objekte jedoch voneinander: Die KochKurve hat unendliche Länge. Denn bei jedem Konstruktionsschritt verlängert sich das Gebilde um den Faktor 4/3. Dennoch befindet sich die Koch-Kurve auf einer begrenzten Fläche.

Die Selbstähnlichkeit gab Anlass, den Begriff der Dimension unabhängig von topologischen Eigenschaften zu fassen. Es widerstrebt der Intuition, einer unendlichen langen Kurve auf begrenzter Fläche dieselbe Dimension zuzuweisen wie einer Strecke. Die Selbstähnlichkeitsdimension beruht auf der Beobachtung, dass auch nicht-fraktale Objekte selbstähnlich sein können und es einen Zusammenhang zwischen Verkleinerungsfaktor und Anzahl der verkleinerten Teile gibt. Verkleinert man z.B. einen Würfel mit dem Faktor $s$, so kann man den ursprünglichen Würfel aus $1 / s^{3}$ kleinen Würfeln wieder zusammensetzen. Definiert man als Selbstähnlichkeitsdimension den Exponenten $D$ des Zusammenhangs

$$
a=\frac{1}{s^{D}}
$$

so erhält man für eine Strecke $D=1$, für ein Quadrat $D=2$ und für einen Würfel eben $D=3$, also die gewohnten Überdeckungsdimensionen. Für diese Objekte ist der Verkleinerungsfaktor beliebig. Bei der Koch-Kurve ist er hingegen durch die Konstruktion festgelegt, nämlich 1/3. Die Anzahl der Teile, aus der man die Koch-Kurve wieder zusammensetzen kann, ist vier. Damit gilt Beziehung 3.19, da $4=3^{D}$. Löst man dies nach $D$ auf, erhält man als Selbstähnlichkeitsdimension für die Koch-Kurve $D=\ln 4 / \ln 3 \approx 1,2619$.

Die solchermaßen direkte Berechnung ist nur bei Konstrukten wie der Koch-Kurve möglich. Doch auch nicht exakt selbstähnlichen Strukturen lässt

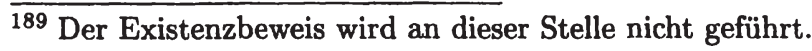


sich bisweilen keine ganzzahlige Dimension sinnvoll zuordnen. Das Ergebnis der Vermessung einer Küstenlinie beispielsweise hängt stark von der Auflösung der zugrundegelegten Karten ab. Die empirisch ermittelte Dimension der Küstenlinie von Großbritannien beträgt 1,36. Eine sinnvolle Länge kann man ihr nicht zuordnen. ${ }^{190}$

Mittlerweile ist eine ganze Reihe von Dimensionsbegriffen eingeführt worden, die alle miteinander verwandt sind. ${ }^{191}$ Sie sind nicht in allen Fällen sinnvoll, manchmal stimmen sie überein, manchmal nicht. ${ }^{192}$ Angeregt wurde die Entwicklung verschiedener Dimensionen von der grundlegenden Arbeit von Hausdorff, der die Überdeckungsdimension verallgemeinerte. ${ }^{193}$ Die Hausdorff-Dimension $D_{H}$ einer Menge $A \subset \mathbb{R}^{n}$ beruht auf dem $s$ dimensionalen Hausdorff-Maß:

$$
h^{s}(A)=\liminf _{\epsilon \rightarrow 0}\left\{\sum_{i=0}^{\infty} \operatorname{diam}\left(U_{i}\right)^{s}\right\} .
$$

Die $U_{i}$ sind offene Überdeckungen von $A$ mit Durchmessern diam $\left(U_{i}\right)<\epsilon$, die in die $s$-te Potenz erhoben werden. Die Durchmesser sind definiert als

$$
\operatorname{diam}(U)=\sup \{\|x-y\| \text { für alle } x, y \in U\}
$$

mit $\|\cdot\|$ als Euklidischer Norm. Die Reihen sind endlich oder unendlich. Für jede Menge $A$ gibt es nun eine Zahl $D_{H}(A)$, so dass

$$
h^{s}(A)= \begin{cases}0 & \text { für } s>D_{H}(A) \\ \infty & \text { für } s<D_{H}(A) .\end{cases}
$$

Die Hausdorff-Dimension $D_{H}(A)$ ist definiert als

$$
D_{H}(A)=\inf \left\{s \mid h^{s}(A)=0\right\}=\sup \left\{s \mid h^{s}(A)=\infty\right\} .
$$

Bei der konkreten Berechnung der Hausdorff-Dimension gibt es eine Reihe von Schwierigkeiten, die die Entwicklung der Box-Dimension $D_{b}$ motiviert haben. Die beiden Dimensionsbegriffe sind nicht äquivalent, stimmen jedoch für eine große Klasse von Fraktalen überein. ${ }^{194}$ Die größte Schwierigkeit der Hausdorff-Dimension liegt in der Bestimmung der Ausdrücke

\footnotetext{
190 Vgl. Mandelbrot (1967).

191 Vgl. Peitgen, Jürgens und Saupe (1992), S. 245.

${ }^{192}$ Vgl. zu den Einzelheiten z.B. Falconer (1990) und Farmer, Ott und Yorke (1983).

193 Vgl. Hausdorff (1918).

194 Vgl. Peitgen, Jürgens und Saupe (1992), S. 264.
} 
$\sum_{i=0}^{\infty} \operatorname{diam}\left(U_{i}\right)^{s}$. Die Box-Dimension ersetzt $\operatorname{diam}\left(U_{i}\right)^{s}$ durch $\epsilon^{s}$. Es sind also nicht mehr beliebige Überdeckungen erlaubt, sondern nur noch solche mit Durchmesser diam $\left(U_{i}\right)=\epsilon$. Die Definitionen als Grenzwerte für $\epsilon \rightarrow 0$ gewährleisten in den meisten Fällen Übereinstimmung der beiden Dimensionen.

Der Vorteil der Boxdimension liegt in der leichten Berechenbarkeit. Dazu unterteilt man eine Teilmenge von $\mathbb{R}^{n}$, die die Menge $A$ ganz enthält, in Kuben der Seitenlänge $\epsilon$ und zählt, wie viele dieser Kuben Elemente aus $A$ enthalten. Diese Zahl heißt $N_{\epsilon}(A)$. Es gilt ${ }^{195}$

$$
D_{b}(A)=\lim _{\epsilon \rightarrow 0} \frac{\log N_{\epsilon}(A)}{\log 1 / \epsilon} .
$$

Diese Definition besagt, dass $N_{\epsilon}(A) \propto \epsilon^{s}$. Der Ausdruck $N_{\epsilon}(A) \epsilon^{-s}$ übernimmt die Rolle des Hausdorff-Maßes. Die Zählung wird für verschiedene $\epsilon$ durchgeführt, so dass man die Boxdimension als Proportionalitätsfaktor berechnen kann. Der Algorithmus ist auch für empirische Daten anwendbar, hier muss der Proportionalitätsfaktor geschätzt werden, wofür sich die Methode der kleinsten Quadrate anbietet.

Eine Schwäche der Boxdimension besteht darin, dass bei der Zählung der Kuben unbeachtet bleibt, dass in einem Kubus mehrere Elemente aus $A$ liegen können. Man überwindet diesen Mangel, indem man die Kuben entsprechend gewichtet. Die Gewichtung erfolgt über das natürliche $\mathrm{Maß} \mu$. Wir betrachten den Attraktor $A$ eines Systems $\mathbf{x}_{k+1}=\mathbf{f}\left(\mathbf{x}_{k}\right)$. Das natürliche Maß einer Menge $B \subset \mathbb{R}^{n}$ ist

$$
\mu(B)=\lim _{n \rightarrow \infty} \frac{1}{n+1} \sum_{k=0}^{n} \mathbf{1}_{B}\left(\mathbf{x}_{k}\right)
$$

mit

$$
\mathbf{1}_{B}(x)= \begin{cases}1 & \text { für } x \in B \\ 0 & \text { sonst. }\end{cases}
$$

Für stetige Systeme $\dot{\mathbf{x}}=\mathbf{f}(\mathbf{x})$ definiert man das natürliche Maß analog:

$$
\mu_{B}=\lim _{t \rightarrow \infty} \frac{1}{t} \int_{0}^{t} \mathbf{1}_{B}(\mathbf{x}(\tau)) d \tau
$$

Da $\mu(B)$ den Anteil aller $\mathbf{x}_{k}$ angibt, die in $B$ fallen, kann man

$$
I(\epsilon)=\sum_{k=1}^{N(\epsilon)} \mu\left(B_{k}\right) \log _{2} \frac{1}{\mu\left(B_{k}\right)}
$$

$\overline{195}$ Vgl. Peitgen, Jürgens und Saupe (1992), S. 264. 
als Information im Sinne der Informationstheorie von Shannon begreifen. ${ }^{196}$ Eine Verkleinerung des Kubusdurchmessers $\epsilon$ vergrößert die Information. Für $\epsilon \rightarrow 0$ wächst $N(\epsilon)$ mit $1 / \epsilon$ logarithmisch an. Die Informationsdimension wird definiert als

$$
D_{I}=\lim _{\epsilon \rightarrow 0} \frac{I(\epsilon)}{\log _{2}(1 / \epsilon)} .
$$

Man kann zeigen, dass $D_{I} \leq D_{b}{ }^{197}$ Es sei noch vermerkt, dass man Boxdimension und Informationsdimension in ein Spektrum von Dimensionen, die sogenannten Rényi-Dimensionen einbetten kann, indem man die RényiInformation $q$-ter Ordnung

$$
I_{q}(\epsilon)=\frac{1}{1-q} \log _{2} \sum_{k=1}^{N(\epsilon)} \mu\left(B_{k}\right)^{q}
$$

verwendet. Die Rényi-Dimension $q$-ter Ordnung ist dann

$$
D_{q}=\lim _{\epsilon \rightarrow 0} \frac{I_{q}(\epsilon)}{\log _{2}(1 / s)} .
$$

Die Boxdimension entspricht $D_{0}$, die Informationsdimension $D_{1}$.

Die Informationsdimension berücksichtigt zwar das natürliche $\mathrm{MaB}$ der Kuben, das Problem der praktischen Berechnung besteht jedoch weiterhin. Nach einer Vermutung von Kaplan und Yorke gibt es nun einen Zusammenhang zwischen Informationsdimension und den Lyapunov-Exponenten eines Systems. In $\mathbb{R}^{n}$ hat man $n$ der Größe nach geordnete Lyapunov-Exponenten $\lambda_{1} \geq \cdots \geq \lambda_{n}$. Die Lyapunov-Dimension $D_{L}$ wird definiert als

$$
D_{L}= \begin{cases}0 & \text { für } m=0 \\ m+\frac{1}{\left|\lambda_{m+1}\right|} \sum_{k=1}^{m} \lambda_{k} & \text { für } 0<m<n \\ n & \text { für } m=n,\end{cases}
$$

wobei $m$ die größte ganze Zahl ist mit $\sum_{k=1}^{m} \lambda_{k} \geq 0$. Die Vermutung von Kaplan und Yorke besagt, dass ${ }^{198}$

$$
D_{L}=D_{I} .
$$

Die Vermutung wurde bislang nicht streng bewiesen, doch in vielen Untersuchungen und numerischen Experimenten belegt, so dass eine Anwendung der Lyapunov-Dimension aufgrund der im Verhältnis zur Informationsdimension guten Berechenbarkeit von Lyapunov-Exponenten als sinnvoll erscheint.

196 Vgl. Shannon (1948).

197 Vgl. Peitgen, Jürgens und Saupe (1992), S. 308.

${ }^{198}$ In Kaplan und Yorke (1979) wurde noch angenommen, dass $D_{L}=D_{b}$. Dies wurde später aufgegeben und durch die Vermutung $D_{L}=D_{I}$ ersetzt. Vgl. Frederickson, Kaplan, Yorke und Yorke (1983). 


\subsubsection{Zwei seltsame Attraktoren}

Um ein Bild von der Ästhetik und dem Detailreichtum seltsamer Attraktoren zu vermitteln, werden hier zwei Beispiele vorgestellt: der Hénon-Attraktor ${ }^{199}$ und der Lorenz-Attraktor ${ }^{200}$. Wie die meisten seltsamen Attraktoren gibt es auch bei diesen beiden zahlreiche ungelöste Fragen. Wir werden allerdings weder diesen Fragen nachgehen, noch die bislang gewonnenen Erkenntnisse im Einzelnen nachzeichnen.

Der Hénon-Attraktor beruht auf dem diskreten, zweidimensionalen System

$$
H(x, y)=\left(\begin{array}{c}
y+1-a \cdot x^{2} \\
b \cdot x
\end{array}\right)
$$

das eine Erweiterung der logistischen Parabel in zwei Dimensionen darstellt. Setzt man in Gleichung (3.20) $b=0$, ergibt sich ein der logistischen Parabel nach Gleichung (3.8) äquivalenter Iterator. ${ }^{201}$ Für die Parameterwerte $a=$ 1,4 und $b=0,3$ erhält man den in Abbildung 3.15 dargestellten Attraktor.

Bei oberflächlicher Betrachtung erweckt der Hénon-Attraktor den Eindruck, als sei er aus wenigen, parabelähnlichen Kurven zusammengesetzt. Ein Zoom in das Kästchen der Abbildung 3.15 offenbart die fraktale Struktur des Attraktors. In Abbildung 3.16 wird deutlich, dass der Attraktor aus vielen Schichten besteht. Je weiter man den Bildausschnitt vergrößert, desto mehr Schichten kommen zum Vorschein - es gibt unendlich viele. Dies ist eine Folge des nichtlinearen Terms in Gleichung (3.20); die Geometrie der Gleichung lässt sich am besten als Strecken und Falten beschreiben. ${ }^{202}$ Der Attraktor hat eine fraktale Struktur, in Abbildung 3.16 ist eine Struktur ähnlich einer Cantormenge erkennbar.

Die Dynamik auf dem Attraktor ist in der Tat chaotisch. Die in Abbildung 3.17 oben dargestellten Bahnen für $x_{t}$ und $y_{t}$ zeigen das typische, scheinbar regellose Muster, das von der logistischen Parabel her vertraut ist. Im unteren Teil von Abbildung 3.17 ist die Entwicklung der Differenz zweier Bahnen dargestellt, deren Startwerte um weniger als 0,001 auseinander liegen. Die sensitive Abhängigkeit von den Anfangsbedingungen ist gut zu erkennen.

Die Berechnung von Lyapunov-Spektrum und Lyapunov-Dimension bestätigt die augenscheinlichen Ergebnisse. Man errechnet das Lyapunov-

\footnotetext{
199 Vgl. Hénon (1976).

200 Vgl. Lorenz (1963).

201 Die Äquivalenz ist durch Variablentransformation leicht zu beweisen. Vgl. Peitgen, Jürgens und Saupe (1994), S. 234f.

202 Vgl. hierzu ausführlich Peitgen, Jürgens und Saupe (1994), S. 218ff.
} 


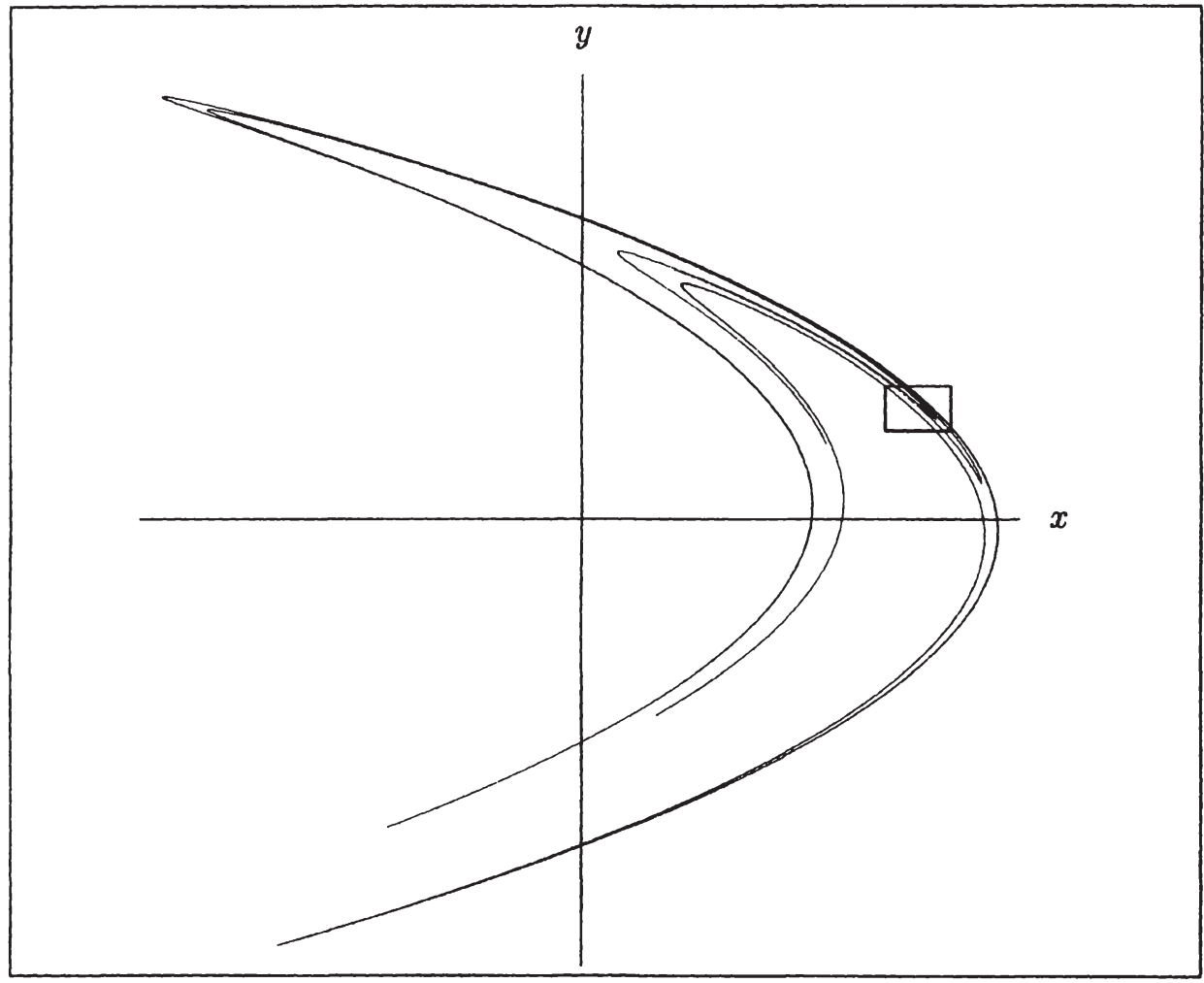

Abbildung 3.15: Hénon-Attraktor

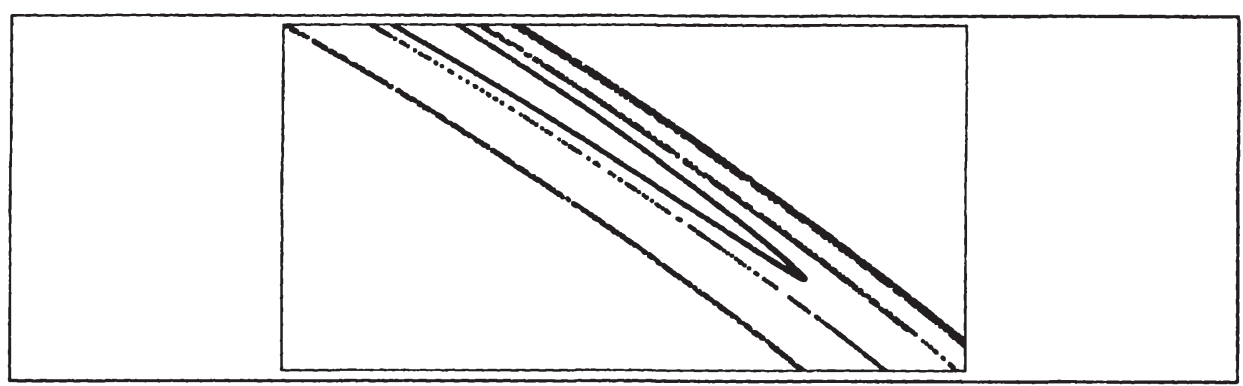

Abbildung 3.16: Hénon-Attraktor - Detailansicht

Spektrum $\left\{\lambda_{1}, \lambda_{2}\right\}=\{0,42,-1,62\}$ und die Lyapunov-Dimension $D_{L}=$ 1,25. Ein positiver Lyapunov-Exponent zeigt die Sensitivität an, das gebrochene Dimensionsmaß die fraktale Struktur des Attraktors. ${ }^{203}$

${ }^{203}$ Ausführlichere Diskussionen des Hénon-Systems liefern Peitgen, Jürgens und Saupe (1994), S. 217ff., und Strogatz (1994), S. 429ff. 


$$
x, y
$$

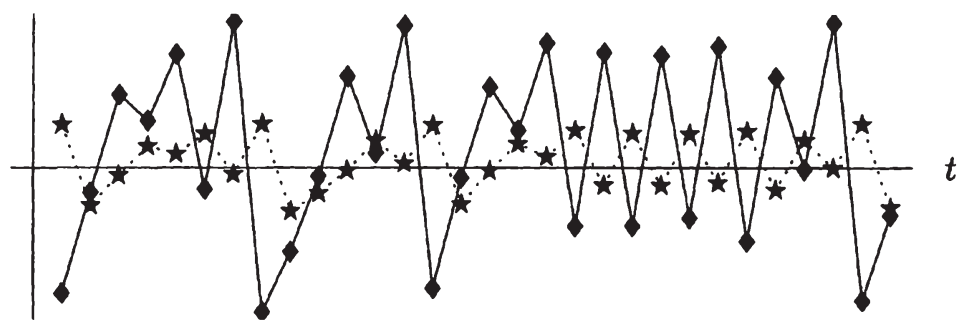

$d$

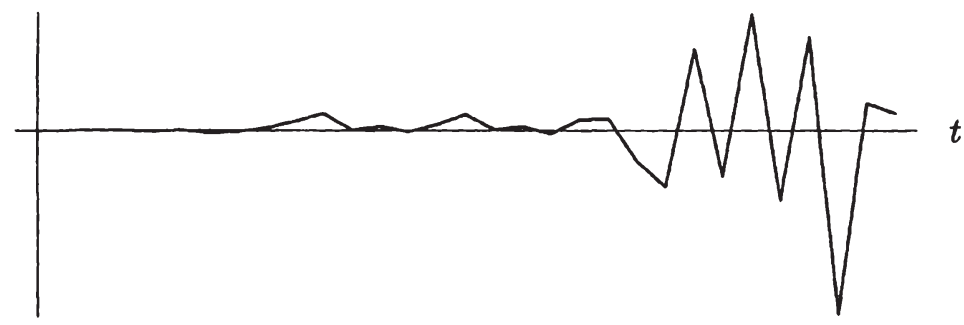

Abbildung 3.17: Bahnen auf dem Hénon-Attraktor

Der Lorenz-Attraktor ist gleichsam der Ausgangspunkt der Chaosforschung. ${ }^{204}$ Das Differentialgleichungssystem

$$
\left(\begin{array}{c}
\dot{x} \\
\dot{y} \\
\dot{z}
\end{array}\right)=\left(\begin{array}{c}
-\sigma(-x+y) \\
r x-y-x z \\
-b z+x y
\end{array}\right)
$$

stellt ein stark vereinfachtes Modell von thermischer Konvektion dar, d.h. von senkrechten Bewegungen von Luftmassen durch Hitzeeinstrahlung. Die Gleichungen beschreiben exakt die Bewegung eines Wasserrades, die mit Konvektionen in der Atmosphäre vergleichbar sind. ${ }^{205}$ Der in Abbildung 3.18 dargestellte Attraktor erinnert an einen Schmetterling. Die Parameterwerte betragen $\sigma=10, r=28$ und $b=8 / 3$. Auch hier lassen sich die Sensitivität und die fraktale Struktur mittels Lyapunov-Spektrum und LyapunovDimension nachweisen. Man erhält durch numerische Simulation die Werte $\left\{\lambda_{1}, \lambda_{2}, \lambda_{3}\right\}=\{0,905 ; 0 ;-14,57\}$ und $D_{L}=2,062$.

$\overline{204}$ Vgl. die Ausführungen in Kapitel 2.

205 Vgl. Strogatz (1994), S. 301ff. 
3.6. BIFURKATIONSTHEORIE

85

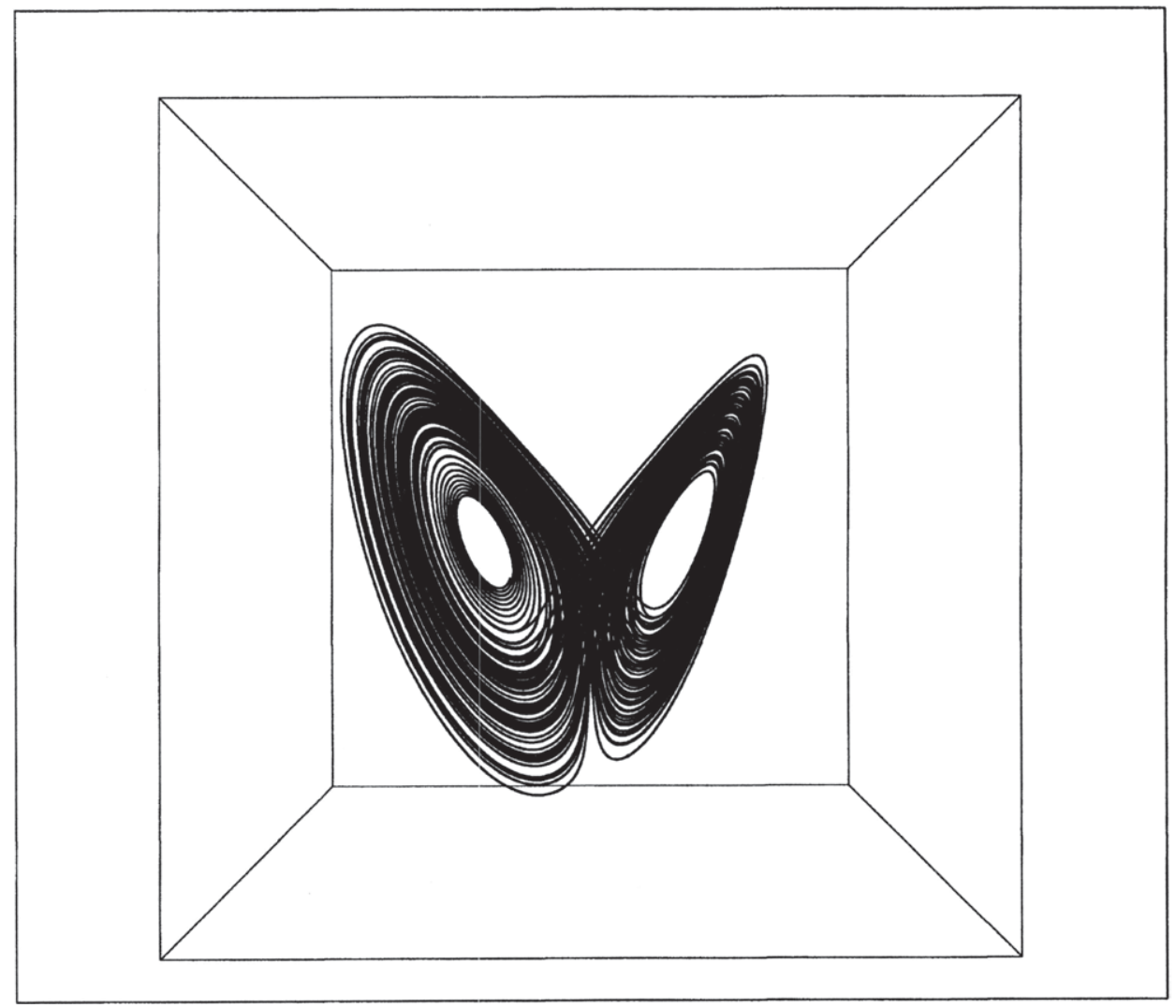

Abbildung 3.18: Lorenz-Attraktor

Dass die Trajektorien chaotisch verlaufen, zeigt Abbildung 3.19. Es ist in keiner Dimension ein Muster erkennbar, weder ein periodischer Grenzzyklus, noch ein Fixpunkt.

3.6 Bifurkationstheorie

3.6.1 Begriff der Bifurkation

Wir haben gesehen, dass die logistische Parabel $x_{t+1}=a \cdot x_{t}\left(1-x_{t}\right)$ topologisch chaotisch für $a=4$ ist und mit Hilfe des Lyapunov-Exponenten untersucht, wie stark die Sensitivität für verschiedene Werte des Parameters $a$ ausgeprägt ist. Es zeigte sich, dass die logistische Parabel je nach Wert von

Lars Otterpohl - 978-3-631-75508-2

Downloaded from PubFactory at 01/11/2019 04:01:56AM

via free access 

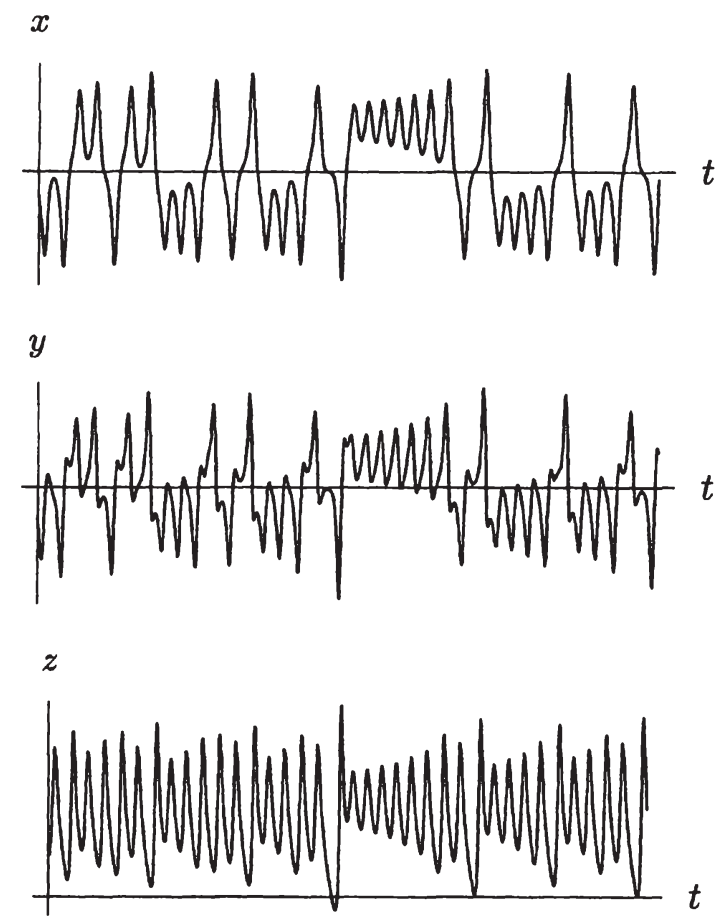

Abbildung 3.19: Trajektorien auf dem Lorenz-Attraktor

$a$ stabiles oder sensitives bzw. chaotisches Verhalten zeigen kann, d.h. Stabilität und Chaos finden sich in demselben Bewegungsgesetz. Es gibt einen wohldefinierten Weg von der Ordnung ins Chaos, der sich an dem Parameter $a$ festmachen lässt. Anhand der logistischen Parabel soll studiert werden, wie sich das Verhalten eines dynamischen Systems mit einem Parameter ändert. Eine solche Verhaltensänderung heißt Bifurkation.

Allgemein werden Familien von reellwertigen Funktionen betrachtet, die von einem Parameter abhängen ${ }^{206}$ :

$$
F(x, a)=f_{a}(x),
$$

wobei $x$ sowohl Skalar als auch Vektor sein kann.

Solche Situationen sind in ökonomischen Modellen allgegenwärtig. Parameter spiegeln exogene Einflüsse wider, die nicht modellimmanent erklärt ${ }^{206}$ Vgl. Devaney (1989), S. 80. 
werden sollen oder können. Im Gegensatz etwa zu physikalischen Modellen gibt es für sie in ökonomischen Modellen in der Regel keine universell gültigen Werte. Oftmals lassen sich solche Werte selbst empirisch nicht oder nur mit großer Ungenauigkeit ermitteln. Es ist nun wünschenswert zu wissen, ob und wie das qualitative Verhalten eines dynamischen Modells von den Parameterwerten abhängt. ${ }^{207}$

Die graphische Darstellung von Bifurkationen erfolgt in FeigenbaumDiagrammen, in denen die Attraktoren auf der Ordinate gegen den Parameterwert $a$ auf der Abszisse abgetragen werden. Das bekannteste Beispiel ist das Feigenbaum-Diagramm in Abbildung 3.20, das die Bifurkationen der logistischen Parabel $f_{a}(x)=a \cdot x(1-x)$ zeigt. Es wird im nächsten Abschnitt ausführlich besprochen. Es gibt aber auch weniger komplizierte FeigenbaumDiagramme, z.B. das des Systems $E_{a}(x)=a \cdot e^{x}{ }^{208}$

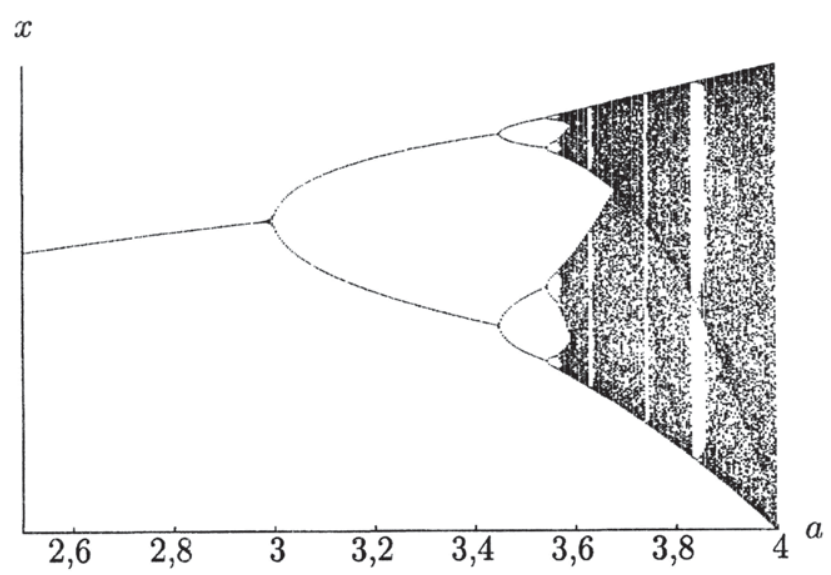

In Anlehnung an: Peitgen, Jürgens und Saupe (1994), S. 138

\section{Abbildung 3.20: Feigenbaum-Diagramm der logistischen Parabel}

Bifurkationen finden sich in der Nähe nicht-hyperbolischer Fix- und periodischer Punkte ${ }^{209}$. Der Gehalt des folgenden Satzes ist: Eine Funktionenfamilie $F(x, a)=f_{a}(x)$ habe für ein bestimmtes $a_{0}$ einen hyperbolischen Fixpunkt. Dann findet sich für Parameterwerte $a$ in der Nähe von $a_{0}$ auch nur jeweils ein Fixpunkt. Damit ändert sich das qualitative Verhalten des 207 Vgl. Lorenz (1993), S. 80.

${ }^{208}$ Vgl. Devaney (1989), S. 86. Dort wird jedoch in Fig. 12.6 im Unterschied zu Abbildung 3.20 auch der instabile Pfad des Fixpunktes jenseits der Bifurkation gezeigt.

${ }^{209}$ Siehe nochmals Definition 3.6 auf Seite 34. 
Systems durch die Parameteränderung nicht, es liegt keine Bifurkation vor. Die Beweisidee beruht darauf, dass man im Feigenbaum-Diagramm einen funktionalen Zusammenhang zwischen $a$ und $x$ findet, d.h. jedem $a$ in der Nähe von $a_{0}$ ist genau ein Fixpunkt zugeordnet. ${ }^{210}$

\section{Satz 3.22}

Sei $f_{a}(x)$ eine in $x$ und $a$ stetig differenzierbare Funktionenfamilie mit dem Parameter $a$. Für ein $a_{0}$ gebe es ein $x_{0}$, so dass

$$
f_{a_{0}}\left(x_{0}\right)=x_{0} \text { und } f_{a_{0}}^{\prime}\left(x_{0}\right) \neq 1
$$

Dann gibt es eine Umgebung $U$ um $a_{0}$ und $V$ um $x_{0}$ und eine stetige Funktion $\phi: U \rightarrow V$, so dass für alle $a \in U$

$$
x_{0}=\phi\left(a_{0}\right) \text { und } f_{a}(\phi(a))=\phi(a) .
$$

Außerdem hat $f_{a}$ keine anderen Fixpunkte in $V$.

Beweis. Wir betrachten die Funktion $G(x, a)=f_{a}(x)-x$. Nach Voraussetzung ist $G\left(x_{0}, a_{0}\right)=0$ und

$$
\frac{\partial G\left(x_{0}, a_{0}\right)}{\partial x}=f_{a_{0}}^{\prime}\left(x_{0}\right)-1 \neq 0
$$

Mit diesen Eigenschaften sind die Voraussetzungen des Satzes über implizite Funktionen ${ }^{211}$ erfüllt, und die Existenz einer stetigen Funktion $\phi: U \rightarrow V$ mit $\phi\left(a_{0}\right)=x_{0}$ und $G(\phi(a), a)=0$ für alle $a \in U$ garantiert. Außerdem ist $G(x, a) \neq 0$ für $x \neq \phi(a)$.

In reellwertigen, eindimensionalen Systemen finden sich zwei Typen der Bifurkation am häufigsten: die Sattelpunktbifurkation und die Periodenverdopplung. Beide sollen hier kurz besprochen werden. Im Anschluss daran wird die Hopf-Bifurkation vorgestellt, die nur in mehrdimensionalen Systemen auftauchen kann.

Um die weitere Analyse zu vereinfachen, benutzen wir folgendes

\section{Lemma 3.23}

Die Funktionenfamilie $f_{a}$ erfülle die Voraussetzungen von Satz 3.22. Dann hat die Funktionenfamilie

$$
g_{a}(z)=f_{a}(z+\phi(a))-\phi(a)
$$

an der Stelle $z=0$ einen Fixpunkt für alle $a$. Die dynamischen Eigenschaften von $f_{a}$ und $g_{a}$ stimmen überein.

210 Vgl. Devaney (1989), S. 84.

211 Vgl. Forster (1993), S. 71. 
Beweis. Aus der Konstruktion von $g_{a}$ folgt

$$
\forall a: g_{a}(0)=f_{a}(\phi(a))-\phi(a)=0 .
$$

Außerdem ist $g_{a}$ topologisch konjugiert zu $f_{a}$ über den Homöomorphismus $h_{a}(x)=x-\phi(a)$.

Mit Hilfe dieses Lemmas können wir uns auf Fixpunkte im Ursprung beschränken und die Ergebnisse auf den allgemeinen Fall übertragen. ${ }^{212}$

\subsubsection{Sattelpunktbifurkation}

Wenn ein System eine Sattelpunktbifurkation erfährt, entstehen zwei Fixpunkte, ein Attraktor und ein Repellor. Der folgende Satz fasst die Voraussetzungen für eine Sattelpunktbifurkation zusammen. Die Idee des Satzes 3.24 liegt darin, die Existenz einer Funktion $\phi: U \rightarrow \mathbb{R}, x \mapsto a$ zu zeigen ${ }^{213}$, die jedem Fixpunkt der Funktionenfamilie $f_{a}$ den zugehörigen Parameterwert $a$ zuordnet. Diese Funktion hat ein Extremum bei $x=0$ mit $\phi(0)=a_{0}$ und für $a>a_{0}$ bzw. $a<a_{0}$ einen stabilen und einen instabilen Ast, wie Abbildung 3.21 links zeigt.

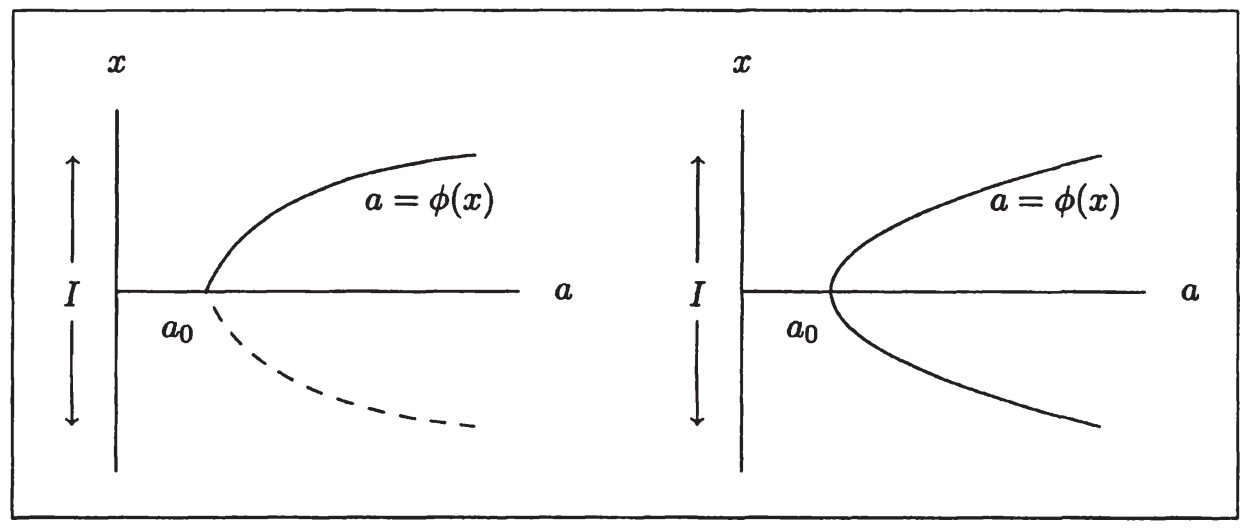

Abbildung 3.21: Sattelpunktbifurkation (links) und Periodenverdopplung (rechts)

\section{Satz 3.24}

Sei $f_{a}: \mathbb{R} \rightarrow \mathbb{R}, x \mapsto f_{a}(x)$ eine in $x$ und $a$ zweimal stetig differenzierbare Funktionenfamilie mit folgenden Eigenschaften:

212 Vgl. Devaney (1989), S. 87.

213 Vgl. Devaney (1989), S. 88. 
(i) $f_{a_{0}}(0)=0$

(ii) $f_{a_{0}}^{\prime}(0)=1$

(iii) $f_{a_{0}}^{\prime \prime}(0) \neq 0$ und $<\infty$

(iv) $\left.\frac{\partial f_{a}(0)}{\partial a}\right|_{a=a_{0}} \neq 0$.

Dann gibt es eine Umgebung $U$ um 0 und eine stetige Funktion $\phi: U \rightarrow \mathbb{R}$ mit $\phi(0)=a_{0}$ und

$$
f_{\phi(x)}(x)=x \text {. }
$$

Außerdem besitzt $\phi$ an der Stelle 0 ein lokales Extremum.

Beweis. Wir definieren $G(x, a)=f_{a}(x)-x$ und wenden darauf den Satz über implizite Funktionen an. Zunächst halten wir fest, dass $G$ stetig partiell differenzierbar in $x$ und $a$ ist. Dies folgt unmittelbar aus der stetigen Differenzierbarkeit von $f_{a}$. Außerdem gilt:

$$
G\left(0, a_{0}\right)=0 \text { und } \frac{\partial G\left(0, a_{0}\right)}{\partial a}=\left.\frac{\partial f_{a}(0)}{\partial a}\right|_{a=a_{0}} \neq 0 .
$$

Es existiert also eine stetige Funktion $\phi(x)$, die $G(x, \phi(x))=0$ genügt. Aus der Kettenregel der Differentiation folgt:

$$
\begin{array}{r}
\frac{\partial G(x, \phi(x))}{\partial x}+\frac{\partial G(x, \phi(x))}{\partial a} \cdot \phi^{\prime}(x)=0 \\
\Longleftrightarrow \phi^{\prime}(x)=-\frac{\frac{\partial G(x, \phi(x))}{\partial x}}{\frac{\partial G(x, \phi(x))}{\partial a}}=-\frac{f_{a_{0}}^{\prime}(x)-1}{\frac{\partial f_{a}(x)}{\partial a}}
\end{array}
$$

so dass $\phi^{\prime}(0)=0$.

Die zweite Ableitung an der Stelle $x=0$ lautet

$$
\phi^{\prime \prime}(0)=-\frac{\frac{\partial^{2} G((0, \phi(0)))}{\partial x^{2}} \cdot \frac{\partial G((0, \phi(0)))}{\partial a}}{\left(\frac{\partial G((0, \phi(0)))}{\partial a}\right)^{2}}=-\frac{f_{a_{0}}^{\prime \prime}(0)}{\left.\frac{\partial f_{a}(0)}{\partial a}\right|_{a=a_{0}}} \neq 0 .
$$




\subsubsection{Periodenverdopplung}

Ein ganz ähnlicher Satz wird nun für den anderen Fall, die Periodenverdopplung gezeigt. Er fasst die Voraussetzungen zusammen, unter denen sich ein Fixpunkt bei Variation des Parameters in einen Zyklus der Periode 2 verzweigt. ${ }^{214}$ Es wird ebenfalls eine Funktion $\phi: U \rightarrow \mathbb{R}, x \mapsto a$ konstruiert, deren Äste jetzt aber für zweiperiodige Zyklen stehen. Abbildung 3.21 stellt diese Funktion auf der rechten Seite dar. Die dort dargestellte parabolische Form der Funktion $\phi$ entsteht, wenn die Schwarzsche Ableitung

$$
S f_{a}(x)=\frac{f_{a}^{\prime \prime \prime}(x)}{f_{a}^{\prime}(x)}-\frac{3}{2}\left(\frac{f_{a}^{\prime \prime}(x)}{f_{a}^{\prime}(x)}\right)^{2}
$$

für $x=0$ und $a=a_{0}$ ungleich Null ist. ${ }^{215}$

\section{Satz 3.25}

Sei $f_{a}: \mathbb{R} \rightarrow \mathbb{R}, x \mapsto f_{a}(x)$ eine in $x$ und $a$ stetig differenzierbare Funktionenfamilie mit folgenden Eigenschaften:

(i) $f_{a}(0)=0$ für alle $a$ in einem Intervall um $a_{0}$

(ii) $f_{a_{0}}^{\prime}(0)=-1$

(iii) $\left.\frac{\partial\left(f_{a}^{2}(0)\right)^{\prime}}{\partial a}\right|_{a=a_{0}} \neq 0$.

Dann gibt es ein Intervall $I$ um 0 und eine Funktion $\phi: I \rightarrow \mathbb{R}$, so dass

$$
f_{\phi(x)}(x) \neq x \quad \text { aber } \quad f_{\phi(x)}^{2}(x)=x .
$$

Beweis. Wir definieren $G(x, a)=f_{a}^{2}(x)-x$ und

$$
H(x, a)= \begin{cases}\frac{G(x, a)}{x} & \text { für } x \neq 0 \\ \frac{\partial G}{\partial x}(0, a) & \text { für } x=0\end{cases}
$$

Im Gegensatz zum vorhergehenden Beweis kann man nicht direkt mit $G$ arbeiten, da $\frac{\partial G\left(0, a_{0}\right)}{\partial a}=0$. Dies ergibt sich aus der Voraussetzung $f_{a}(0)=0$ in einer Umgebung von $a_{0}$, denn damit ändert sich der Funktionswert bei einer infinitesimalen Variation von $a$ in $a_{0}$ nicht.

${ }^{214}$ Vgl. Devaney (1989), S. 90.

215 Vgl. Devaney (1989), S. 69 und $91 f$. 
Wir prüfen nun, ob $H$ an der Stelle $\left(0, a_{0}\right)$ die Voraussetzungen des Satzes über stetige Funktionen erfüllt. Zunächst ergibt sich aus der Regel von de l'Hôpital ${ }^{216}$ :

$$
\lim _{x \rightarrow 0} \frac{G(x, a)}{x}=\lim _{x \rightarrow 0} \frac{\partial G(x, a)}{\partial x}=0 .
$$

Außerdem gilt noch

$$
H\left(0, a_{0}\right)=\frac{\partial G\left(0, a_{0}\right)}{\partial x}=\left(f_{a_{0}}^{2}(0)\right)^{\prime}-1=\left(f_{a_{0}}^{\prime}(0)\right)^{2}-1=0,
$$

d.h. $H$ ist an der Stelle $\left(0, a_{0}\right)$ stetig.

Die Existenz stetiger partieller Ableitungen ist für $x \neq 0$ und $a$ klar. Für $x=0$ berechnen wir

$$
\lim _{x \rightarrow 0} \frac{\partial H(x, a)}{\partial x}=\frac{1}{x^{2}}\left(\frac{\partial G(x, a)}{\partial x} \cdot x-G(x, a)\right) .
$$

Wiederum mit der Regel von de l'Hôpital erhält man daraus

$$
\frac{\partial H(0, a)}{\partial x}=\frac{1}{2} \frac{\partial^{2} G(0, a)}{\partial x^{2}} \text {. }
$$

Gemäß Voraussetzung haben wir schließlich

$$
\frac{\partial G\left(0, a_{0}\right)}{\partial x}=\left.\frac{\partial\left(\left(f_{a}^{2}(0)\right)^{\prime}-1\right)}{\partial a}\right|_{a=a_{0}}=\frac{\partial\left(f_{a}^{2}(0)\right)^{\prime}}{\partial a} \neq 0 .
$$

Also existiert eine stetige Funktion $\phi(x)$ in einer Umgebung von 0 , die $\phi(0)=a_{0}$ und $H(x, \phi(x))=0$ genügt. Damit ist $G(x, \phi(x)) / x=0$ für $x \neq 0$, so dass $x$ ein Punkt der Periode 2 für $f_{\phi(x)}$ ist. Aus Satz 3.22 folgt zudem, dass $x$ kein Fixpunkt für $f_{\phi(x)}$ ist.

\subsubsection{Hopf-Bifurkation}

Die Hopf-Bifurkation tritt in mindestens zweidimensionalen stetigen und diskreten Systemen auf. ${ }^{217}$ Wir können uns hier auf den zweidimensionalen diskreten Fall beschränken. Wenn ein System eine Hopf-Bifurkation durchläuft,

${ }^{216}$ Nach der Regel von de l'Hôpital folgt für zwei in einer Umgebung von $\xi$ differenzierbare Funktionen $f(x)$ und $g(x)$ aus $\lim _{x \rightarrow \xi} f(x)=\lim _{x \rightarrow \xi} g(x)=0$, dass $\lim _{x \rightarrow \xi} f(x) / g(x)=\lim _{x \rightarrow \xi} f^{\prime}(x) / g^{\prime}(x)$. Man kann damit also Grenzwerte von Ausdrücken der Form $0 / 0$ berechnen. Analoge Formeln existieren für Ausdrücke $\infty / \infty$. Vgl. Chiang (1984), S. 429, und Heuser (1994), S. 287.

${ }^{217}$ In diskreten Systemen heißt sie zur Unterscheidung auch Neimark-Sacker-Bifurkation, so etwa in Kuznetsov (1995). 
bedeutet dies, dass ein stabiler Fixpunkt bei Parameteränderung instabil wird und von einer geschlossenen Kurve umgeben wird. Die Kurve zieht alle Bahnen an, gleich ob sie innerhalb oder außerhalb der Kurve starten, außer solche, die im instabilen Fixpunkt beginnen. ${ }^{218}$

Der folgende Satz fasst die Kriterien für eine Hopf-Bifurkation zusammen: ${ }^{219}$

\section{Satz 3.26}

Sei $\mathbf{f}_{a}: \mathbb{R}^{2} \rightarrow \mathbb{R}^{2}, \mathbf{x} \mapsto \mathbf{f}(\mathbf{x})$ eine in $a$ und $\mathbf{x}$ stetig differenzierbare Funktionenfamilie. Die Kurve der Fixpunkte $\mathbf{x}^{*}(a)$ sei stetig differenzierbar mit komplex-konjugierten Eigenwerten $\lambda(a)=\alpha \pm \beta \cdot i$ der Jacobi-Matrix zu $f_{a}$. Es gebe ein $a_{0}$ mit folgenden Eigenschaften:

(i) $|\lambda(a)|=1$,

(ii) $\lambda^{n}(a) \neq 1$ für $n=1,2,3,4$,

(iii) $\left.\frac{d|\lambda(a)|}{d a}\right|_{a=a_{0}}>0$.

Dann gibt es für $a>a_{0}$ eine geschlossene attraktive Kurve um den Fixpunkt $\mathbf{x}^{*}(a)$.

Der Beweis ist sehr lang und technisch und soll hier nicht wiedergegeben werden. ${ }^{220}$ Die Idee besteht darin, die Hopf-Bifurkation für eine sogenannte Normalform ${ }^{221} \mathrm{zu}$ demonstrieren und dann zu zeigen, dass alle Systeme, die die Eigenschaften von Satz 3.26 erfüllen, lokal topologisch äquivalent sind. Die Bedingungen (ii) und (iii) stellen sicher, dass die Bifurkation nicht degeneriert ist. ${ }^{222}$ Bedingung (ii) verlangt, dass in den ersten vier Iterationen die Eigenwerte, die sich unter Iteration immer auf dem Einheitskreis in der komplexen Ebene bewegen, nicht auf der reellen Achse liegen; Bedingung (iii) fordert, dass die Bifurkation bei Parameteränderung mit positiver Geschwindigkeit abläuft, d.h. die Eigenwerte den Einheitskreis mit positiver Geschwindigkeit passieren.

218 Vgl. Kuznetsov (1995), S. 115.

219 Vgl. Lorenz (1993), S. $115 f$.

220 Vgl. Kuznetsov (1995), S. 114ff.

${ }^{221} \mathrm{Vgl}$. Gleichung 4.15 in Kuznetsov (1995), S. 117.

222 Vgl. Kuznetsov (1995), S. 124. 


\subsubsection{Ein Musterbeispiel: Periodenverdopplung in der logistischen Parabel}

Von der Chaostheorie am gründlichsten erforscht ist die Periodenverdopplung der logistischen Parabel $x_{t+1}=a \cdot x_{t}\left(1-x_{t}\right)$. Anhand ihrer lässt sich der Weg von der Ordnung ins Chaos in allen wichtigen Aspekten aufzeigen. ${ }^{223} \mathrm{Im}$ Folgenden wird daher dieser Weg, dessen Darstellung im Feigenbaum-Diagramm eine ähnliche Berühmtheit erlangt hat wie die Mandelbrot-Menge ${ }^{224}$, in der gebotenen Kürze nachgezeichnet ${ }^{225}$.

Die logistische Parabel $x_{t+1}=a \cdot x_{t}\left(1-x_{t}\right)$ besitzt für Parameterwerte $0<a<1$ den stabilen Fixpunkt $(0,0)$, gegen den alle Startwerte des Einheitsintervalls konvergieren. Bei $a=1$ erfährt die Funktion eine Bifurkation. Es entstehen zwei Fixpunkte an den Stellen 0 und $(a-1) / a$, die beide die Fixpunktgleichung $a x(1-x)=x$ erfüllen. Während der erste nur die Urbilder 0 und 1 besitzt und somit abstoßend ist, ist der andere anziehend für das offene Intervall $(0,1)$. Es lässt sich leicht nachprüfen, dass wie in Satz 3.1 vorausgesetzt gilt: $\left|f^{\prime}((a-1) / a)\right|<1$. Dieses Verhalten ist typisch für eine Sattelpunktbifurkation. Wir wollen daher prüfen, ob die Voraussetzungen des Satzes 3.24 erfüllt sind. Man sieht leicht, dass in der Tat $f_{1}(0)=0$, $f_{1}^{\prime}(0)=1$ und $f_{1}^{\prime \prime}(0)=1$. Indes gilt $\partial f / \partial a=0$. Insofern handelt es sich nicht um eine typische Sattelpunktbifurkation. Die partielle Ableitung nach dem Parameter $a$ gibt gleichsam die "Geschwindigkeit" der Verzweigung an. Der Ursprung ist für alle $a \in(0,1)$ Fixpunkt, so dass die Bifurkation mit Geschwindigkeit Null vonstatten geht. ${ }^{226}$

Für $1<a<3$ haben wir es also mit einem Attraktor $(a-1) / a$ und einem Repellor $0 \mathrm{zu}$ tun $^{227}$. Das langfristige Verhalten des Systems ist daher sicher prognostizierbar: Wenn nicht gerade die Startwerte 0 oder 1 gewählt werden,

${ }^{223}$ Das allgemeine Konzept der Bifurkation als Verhaltensänderung eines dynamischen Systems bei Parameteränderung wird umfassender in Lorenz (1993), S. 80ff., diskutiert. Lorenz unterscheidet weitere Arten von Bifurkationen, auch abhängig davon, ob es sich um diskrete oder stetige Systeme handelt.

224 Vgl. z.B. Peitgen, Jürgens und Saupe (1994), S. 435.

${ }^{225}$ Sehr viel ausführlicher ist die Darstellung in Peitgen, Jürgens und Saupe (1994), S. $131 \mathrm{ff}$.

${ }^{226}$ Anders liegt der Fall bei der Sattelpunktbifurkation der Funktionenfamilie $f_{a}(x)=a e^{x}$ für $a=1 / e$. Ist $a>1 / e$, hat die Funktion gar keinen Fixpunkt, bei $a=1 / e$ einen von links anziehenden, von rechts aber abstoßenden Fixpunkt und bei $a<1 / e$ zwei Fixpunkte, einen anziehenden und einen abstoßenden. Die Bifurkation verläuft hier mit ,negativer Geschwindigkeit“, was sich auch in der partiellen Ableitung nach dem Parameter widerspiegelt.

${ }^{227} \mathrm{Zu}$ den Begriffen Attraktor und Repellor vgl. nochmals Definition 3.5 auf S. 34 . 
wird das System gegen den Wert $(a-1) / a$ konvergieren, wie Abbildung 3.22 zeigt. ${ }^{228}$ Der Schnittpunkt der Parabel mit der Winkelhalbierenden kann links oder rechts vom Extremum liegen.

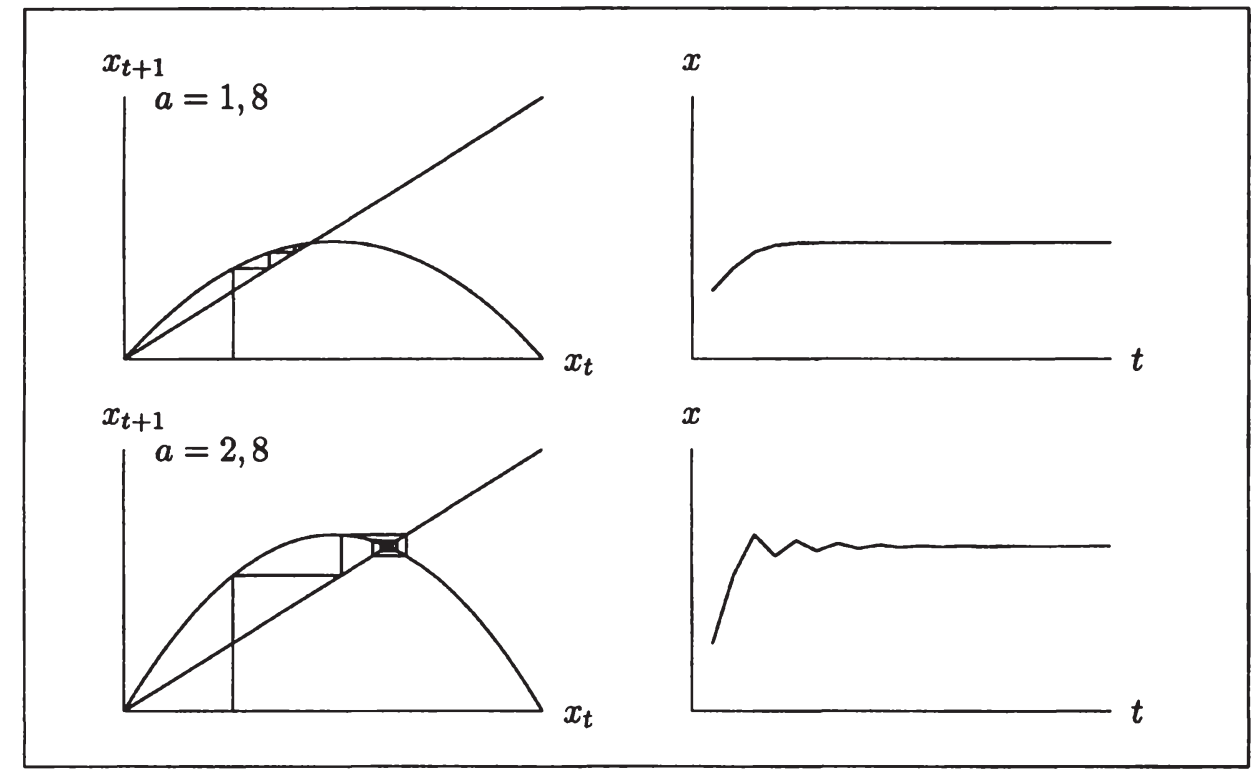

Abbildung 3.22: Phasendiagramm und Zeitreihe für die logistische Parabel

Wenn der Parameter den Wert $a=3$ erreicht, ist der Fixpunkt $(a-1) / a$ nicht mehr hyperbolisch, d.h. $\left|f_{3}^{\prime}(2 / 3)\right|=1$ nach Definition 3.6, ein Zeichen für eine Bifurkation. Um die Voraussetzungen des Satzes 3.25 überprüfen zu können, müssen wir zu der Funktion

$$
g_{a}(z)=f_{a}\left(z+\frac{a-1}{a}\right)-\frac{a-1}{a}
$$

übergehen. Damit wird gemäß Lemma 3.23 der Fixpunkt $(a-1) / a$ in den Ursprung verschoben. Wir erhalten dann für $g_{3}(z)=-3 z^{2}-z$ die Ergebnisse $g_{3}(0)=0, g_{3}^{\prime}(0)=-1$ und $\left.\frac{\partial\left(g_{a}^{2}\right)^{\prime}}{\partial a}\right|_{a=3}(0)=2$. Damit ist nachgewiesen, dass für $a=3$ eine Periodenverdopplung auftritt. Der Fixpunkt $(a-1) / a$ existiert weiterhin, wird aber abstoßend. Es tritt nun ein zweiperiodiger Orbit $\left\{x_{t}, x_{h}\right\}$ als Attraktor auf, der die Gleichung $f_{a}^{2}(x)=x$ erfüllt. Man erhält als Lösungen dieser Gleichung die Wurzeln $x_{h, t}(a)=\frac{a+1 \pm \sqrt{a^{2}-2 a-3}}{2 a}$. Diese

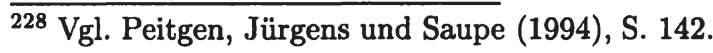



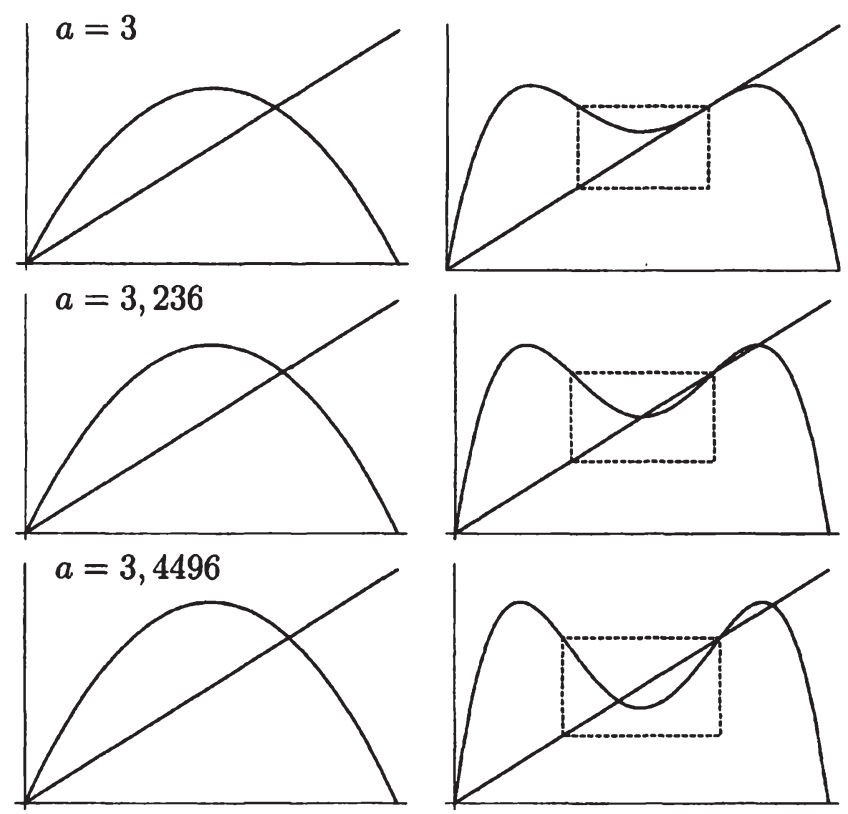

In Anlehnung an: Peitgen, Jürgens und Saupe (1994), S. 161

Abbildung 3.23: Bifurkation in der logistischen Parabel

Lösungen sind nur für $a \geq 3$ definiert und für $a=3$ gleich. Auch hieran kann man sehen, dass ein Orbit der Periode 2 nur für $a>3$ existiert. ${ }^{229}$ Die weitere Entwicklung bei steigendem $a$ wird in der Abbildung 3.23 dokumentiert. Hier sind neben den Graphen von $f_{a}$ auch die Graphen von $f_{a}^{2}$ dargestellt. Die gestrichelten Quadrate in den Graphen zeigen umgedrehte Parabeln, die den Graphen von $f_{1}$ bis $f_{3}$ entsprechen. Folglich erhalten wir analoge Ergebnisse. Für $a=3$ verliert der Fixpunkt $(a-1) / a$ seine Stabilität. Der Graph von $f_{3}^{2}$ zeigt die „atypische“ Sattelpunktbifurkation in dem gestrichelten Quadrat. $a=3,236$ ist der superattraktive Fall; bei $a=3,4496$ verliert der zweiperiodige Zyklus schließlich seine Stabilität und ein Zyklus der Periode 4 entsteht. Wenn der Parameter weiter vergrößert wird, geht die Stabilität erneut verloren, so dass man einen Zyklus der Periode 8 erhält und so weiter. Betrachten wir die Folge $\left\langle b_{n}\right\rangle$ von periodenverdoppelnden Parameterwerten etwas genauer. Die ersten Werte sind 3, 3,4495, 3,5441, 3,5644, 3,5688. Die Differenzen nehmen schnell ab, so dass die Vermutung naheliegt,

$\overline{229}$ Vgl. Peitgen, Jürgens und Saupe (1994), S. 156. 
die Folge $\left\langle d_{n}\right\rangle=\left\langle b_{n}-b_{n-1}\right\rangle$ sei geometrisch. ${ }^{230}$ Dies trifft nicht exakt zu, die Verhältnisse $d_{k} / d_{k+1}$ konvergieren aber gegen den Wert $4,6692 \ldots$, die sog. Feigenbaum-Konstante. Diese Zahl ist überraschenderweise universell, d.h. sie gilt nicht nur für den speziellen Fall der logistischen Parabel, sondern für eine große Klasse von Iteratoren. ${ }^{231}$

Durch das annähernd geometrische Verhalten der Periodenverdopplungspunkte gibt es einen kritischen Wert, jenseits dessen sich die Periodenlängen nicht weiter verdoppeln. Er heißt Feigenbaum-Punkt und hat den Wert $a=3,5699 \ldots$ Dadurch wird das Feigenbaum-Diagramm in zwei Bereiche unterteilt. Für $1<a<3,5699 \ldots$ finden wir den Periodenverdopplungsbaum. Der Bereich 3,5699 $\ldots<a \leq 4$ ist von Chaos bedeckt, das jedoch kein vollständiges Chaos im Sinne von Definition 3.7 ist. Vielmehr lassen sich reichhaltige Strukturen entdecken, denen hier noch ein Stück weit nachgegangen wird.

Betrachten wir das Feigenbaum-Diagramm, Abbildung 3.20 auf S. 87, von rechts nach links, d.h. für fallende Parameterwerte. Am rechten Ende bei $a=4$ ist keine Struktur mehr zu erkennen; im Endzustand wird das Einheitsintervall ganz und gleichmäßig ausgefüllt. Darin spiegelt sich wider, was in Satz 3.9 auf Seite 44 bewiesen wurde: Die logistische Parabel ist für $a=4$ chaotisch. Gehen wir nach links im Diagramm, so wird das Band der Endzustände enger, es wird von dichteren Linien durchzogen, so dass die Punkte des Endzustandes nicht gleichmäßig auf einer vertikalen Linie verteilt sind. Mehrfach wird das Band von sogenannten Fenstern durchbrochen, in denen der Endzustand auf wenige Punkte zusammenfällt. Bei $a \approx 3,6785$ spaltet es sich in zwei Bänder auf, die sich bei $a \approx 3,592$ in vier Bänder teilen und so fort. Man erhält so eine Folge von Parametern, an denen sich die Bänder in doppelt so viele Bänder aufspalten. Diese Folge ist recht bemerkenswert, denn sie wird von den gleichen Eigenschaften beherrscht wie die Folge der periodenverdoppelnden Parameter im linken Teil des Feigenbaum-Diagramms. Beide konvergieren gegen den Feigenbaum-Punkt, und in beiden konvergieren die Verhältnisse der Differenzen aufeinanderfolgender Glieder gegen die Feigenbaum-Konstante. Insofern kann man die chaotischen Bänder auf der rechten Seite als Spiegelbild des Periodenverdopplungsszenarios auf der linken Seite auffassen. ${ }^{232}$

${ }_{230}$ Vgl. Peitgen, Jürgens und Saupe (1994), S. 163.

231 Vgl. Peitgen, Jürgens und Saupe (1994), S. 167ff. und Kuznetsov (1995), S. $127 \mathrm{ff}$.

232 Vgl. Peitgen, Jürgens und Saupe (1994), S. 181. 
Lars Otterpohl - 978-3-631-75508-2

Downloaded from PubFactory at 01/11/2019 04:01:56AM

via free access 


\section{Kapitel 4}

\section{Koordination}

\subsection{Die Notwendigkeit der Koordination}

Im Folgenden ist zu klären, warum Koordination im Rahmen einer ökonomischen Betrachtungsweise ein unvermeidliches Problem darstellt. Zwei Voraussetzungen sind notwendig, damit das Problem der Koordination auftritt: Arbeitsteilung und Interessenkonflikte. Erst wenn mehrere Menschen die Lösung einer Aufgabe gemeinsam in Angriff nehmen, entsteht die Notwendigkeit der gegenseitigen Abstimmung. Und nur bei einem potenziellen Konflikt zwischen dem Interesse an der Aufgabenlösung und den Eigeninteressen der Beteiligten reicht Koordination über eine bloße Frage der Kommunikation hinaus. Daher wird zunächst das Verhaltensmodell, das auf die Interessen des Einzelnen abhebt, das Modell des Homo oeconomicus, beschrieben. Daran anschließend wird die Rolle der Arbeitsteilung eingehend dargestellt und zum Abschluss des Kapitels werden die wichtigsten in der Organisationstheorie diskutierten Instrumente der Koordination systematisiert.

\subsubsection{Das ökonomische Verhaltensmodell}

Die methodologische Grundlage der Ökonomik ist der methodologische Individualismus. Er besagt, „daß alle sozialen Phänomene, insbesondere das Funktionieren der sozialen Institutionen, immer als das Resultat der Entscheidungen, Handlungen, Einstellungen menschlicher Individuen verstanden werden sollen und daß wir nie mit einer Erklärung aufgrund sogenannter ,Kollektive (...) zufrieden sein dürfen“. ${ }^{233}$ Eine Gruppe, etwa ein Unternehmen,

${ }^{233}$ Popper (1945, 1992), S. 116. 
kann keine eigenen Interessen haben, die nicht auf die Gruppenmitglieder zurückgehen, und ihnen entsprechend als Kollektiv handeln. ${ }^{234}$ Das soll nicht bedeuten, dass ein Unternehmen nicht nach außen als Einheit auftreten kann. Doch muss sich solch geschlossenes Auftreten immer auf Interessen, Entscheidungen und Handlungen von Individuen zurückführen lassen. Das Setzen einer solchen Annahme im Rahmen eines Modells muss als methodologische Vereinfachung verstanden werden: Das Problem, wie sich aus dem Handeln der einzelnen Individuen ein geschlossenes Auftreten des Unternehmens nach außen ergibt, wird als vorab gelöst angenommen.

Wenn einmal die Vorentscheidung zugunsten des methodologischen Individualismus gefallen ist, und damit alles auf das Individuum zurückführbar sein muss, benötigt man ein Modell menschlichen Verhaltens. In der Ökonomik wird das Modell des sogenannten „Homo oeconomicus“ verwendet. Da der Homo oeconomicus nicht nur unter Nicht-Wirtschaftswissenschaftlern eher berüchtigt und Gegenstand zahlreicher Missverständnisse ist, wird die Modellstruktur, so wie sie in dieser Arbeit zugrunde gelegt wird, an dieser Stelle noch einmal herausgearbeitet. ${ }^{235}$

Der Homo oeconomicus soll nicht als Menschenbild verstanden werden in dem Sinne, dass er zur Analyse einzelner Individuen dienen könnte ${ }^{236}$. Vielmehr handelt es sich um ein Konstrukt, das geeignet ist, häufig wiederkehrende und auf viele Individuen zutreffende Situationen zu untersuchen. Das Verhalten eines Individuums wird in Abhängigkeit situativer Einflüsse untersucht, die streng in zwei Kategorien unterschieden werden, nämlich als eine Funktion der Präferenzen und Restriktionen. Die Präferenzen stehen für die individuellen Zielvorstellungen, die Restriktionen bilden die Knappheiten der für die Erreichung der Ziele verfügbaren Mittel ab. ${ }^{237}$ Folglich ist es mit dem Konstrukt des Homo oeconomicus möglich, individuelles Verhalten im Ziel-Mittel-Schema darzustellen.

Die Dichotomisierung der Einflussfaktoren beruht auf einer methodischen Vereinfachung. Genau genommen wird nämlich nicht das Verhalten eines Individuums analysiert, sondern eine Verhaltensänderung als Folge einer Veränderung der situativen Einflussfaktoren. ${ }^{238}$ Die Vereinfachung besteht nun darin, dass die Präferenzen als unveränderlich angesehen werden. Die Ver-

${ }^{234}$ Zur organischen Staatstheorie als Beispiel eines methodologischen Kollektivismus siehe Kirchgässner (2000), S. 24 und 96ff.

${ }^{235}$ Vgl. Kirchgässner (2000), Pies (1993), S. 94ff., Becker (1976), passim.

${ }^{236}$ Vgl. Pies (1993), S. 94.

${ }^{237}$ In der strikten Unterscheidung zwischen Präferenzen und Restriktionen unterscheidet sich die Ökonomik von der traditionellen Soziologie. Vgl. Kirchgässner (2000), S. 13.

${ }^{238}$ Vgl. Pies (1993), S. 95. 
haltensänderung wird als ausschließliche Folge veränderter Restriktionen modelliert. Als Begründung hierfür wird in der Regel angeführt, Präferenzen entstünden im Zuge der Sozialisation eines Menschen und seien daher höchstens langfristig veränderlich. ${ }^{239}$ Zusätzlich wird argumentiert, Präferenzen seien nicht bzw. höchstens indirekt ${ }^{240}$ beobachtbar, wohingegen die Restriktionen, denen menschliches Verhalten unterliegt, relativ leicht ermittelbar seien. ${ }^{241}$ Die Annahme stabiler Präferenzen hat zu Recht unter Ökonomen und noch mehr unter Soziologen Unverständnis hervorgerufen. Jeder kann an sich selbst beobachten, dass sich individuelle Ziele und Vorlieben im Laufe der Zeit eben doch verändern, auch in fortgeschrittenem Lebensalter, wenn die Sozialisation als abgeschlossen betrachtet werden kann. Die Annahme gewinnt erst dann an Plausibilität, wenn man sich Beckers Präferenzbegriff zu eigen macht. Dieser bezieht sich eben nicht auf Güter und Dienstleistungen, sondern auf grundlegende Ziele wie Gesundheit, Prestige, Wohlwollen, Neid oder Ähnliches ${ }^{242}$, auf die ,letzten Ziele“. Auf diese Weise erfolgt eine Trennung in Ziele und Mittel dergestalt, dass die instrumentalistische Mittelanalyse auf all jene Zwecke ausgeweitet wird, die sich als Mittel zur Erreichung der letzten Ziele oder Wertmaßstäbe, in der Sprache Max Webers der Ideale ${ }^{243}$, ausweiten lässt. Insofern ist der Präferenzbegriff äquivalent zu inhaltsleeren Formalzielen. Ersteres macht den Homo oeconomicus, Letzteres das Ziel-Mittel-Schema allgemein anwendbar. ${ }^{244}$ Die Abgrenzung von Präferenzen und Restriktionen ist in Beckers Konzept nicht der Widerschein der Trennung von Innen- und Außenwelt des Individuums ${ }^{245}$. Becker identifiziert vielmehr das Invariante und Universelle mit den Präferenzen und nennt alles Veränderliche Restriktionen. Mit diesem Kunstgriff ist die Konstanz der Präferenzen, die somit zwar das Verhalten, nicht aber Verhaltensänderungen beeinflussen, per Definition sichergestellt. Pies merkt hierzu an: „Es entbehrt folglich nicht einer gewissen Ironie, daß ausgerechnet der Becker-Ansatz die Perspektive für eine ökonomische Analyse des Präferenzenwandels eröffnet präziser: des Wandels jener Phänomene, die in der Umgangssprache gemeinhin als Präferenzen bezeichnet werden.“246

${ }^{239}$ Vgl. Kirchgässner (2000), S. 26, Blankart (1998), S. 11.

${ }^{240}$ Zur Theorie der offenbarten Präferenzen vgl. Varian (1992), S. 131ff. Der Ansatz geht zurück auf Samuelson (1948).

241 Vgl. Kirchgässner (2000), S. $25 f$.

242 Vgl. Becker (1976), S. 4.

${ }^{243}$ Vgl. Weber (1922, 1967), S. 151.

${ }^{244}$ Vgl. Pies (1993), S. 98

${ }^{245}$ Schon Simon (1955), S. 101, betont, dass eine solche Trennung dazu führt, dass ein Teil der Restriktionen im Individuum selbst begründet liegt.

${ }^{246}$ Pies (1993), S. 99, Fn. 30. 
$\mathrm{Zu}$ welchem Zweck macht man sich aber die Mühe, in unveränderliche Präferenzen und veränderliche Restriktionen $\mathrm{zu}$ unterscheiden, wenn man dafür einen sehr abstrakten Präferenzbegriff in Kauf nehmen muss? Der Grund ist methodologischer Natur. Bei der Untersuchung individueller Verhaltensänderungen stellt sich ein Zurechnungsproblem. Wie lässt sich eine Verhaltensänderung auf Änderungen der Argumente der Verhaltensfunktion zurechnen? Die ökonomische Analyse benutzt hier die ceteris-paribus-Klausel, d.h. die Wirkung der Veränderung einer einzigen Restriktion wird isoliert untersucht; alle anderen Einflussfaktoren werden konstant gehalten. Die Anwendung der ceteris-paribus-Klausel setzt aber unabdingbar voraus, dass sich nicht die individuellen Ziele ändern, sondern nur die Mittel zur Verfolgung dieser Ziele. Damit wird die Annahme stabiler Präferenzen zu einer Heuristik, die das kausale Zurechnungsproblem löst. Theorien können nicht dadurch gegen empirische Widersprüche immunisiert werden, dass auf die Ad-hoc-Erklärung einer Präferenzänderung zurückgegriffen wird. ${ }^{247}$

Das dargelegte Homo-oeconomicus-Modell erlaubt alle denkbaren Ziele und Mittel. Eine solch breite Öffnung sichert eine allgemeine Anwendbarkeit, die Becker für sein Forschungsprogreamm des ökonomischen Imperialismus benötigte. Andererseits verbietet diese Allgemeinheit jedoch zunächst, anhand des Modells die Entscheidungen eines Individuums vorauszusagen. An Prognosekraft gewinnt das Modell erst durch die Annahme der Rationalität individuellen Verhaltens: Individuen kennen ihre Interessen und Ziele und wählen Handlungen nach deren Beiträgen zu den Zielen aus. Die übliche Analysemethode der Ökonomik ist der Nutzenmaximierungskalkül. Der Mitteleinsatz erfolgt so, dass ein individuelles Nutzenmaximum erreicht wird, das sich als Gleichgewicht interpretieren lässt. Verhaltensänderungen führen nicht mehr zu Nutzensteigerungen, das Individuum hat folglich keinen Anlass, seine Mittel umzudisponieren. Ein solcher Zustand der Ruhe, in dem ohne Anstöße von außen keine Veränderungen zu erwarten sind, wird als methodisches Gleichgewicht bezeichnet. ${ }^{248}$

Die in Kapitel 5 zu diskutierenden Modelle der Koordination verwenden Nutzen- und Gewinnmaximierungskalküle. Das Problem der Veränderbarkeit von Präferenzen wird dabei keine explizite Rolle mehr spielen, weil die interessierenden Phänomene gerade modellendogen ohne Rückgriff auf derartige exogene Einflüsse erklärt werden sollen. Insofern ist die Annahme konstanter Präferenzen auch bezüglich nicht letzter Ziele als Anwendung der ceterisparibus-Klausel anzusehen.

247 Vgl. Pies (1993), S. 97f., Becker (1976), S. 4.

248 Vgl. Felderer und Homburg (1994), S. 12. 
Gewöhnlich wird bezüglich der Ziele unterstellt, Individuen handelten eigennützig. Altruistisches oder bösartiges Verhalten wird damit nicht grundsätzlich ausgeschlossen und in manchen Modellen sogar ausdrücklich angenommen. ${ }^{249}$ Nimmt man reinen Eigennutz an, enthält die Nutzenfunktion nicht den Nutzen anderer Individuen als Argument. Rawls bezeichnet dies als ,mutually disinterested rationality“. 250

Der in Lehrbüchern dargestellte Extremfall des Homo oeconomicus ist der Popanz eines vollständig eigensüchtigen und in jedem Augenblick gleichsam wie ein Computer optimal handelnden Menschen. Dieses Modell hat zu Recht immer wieder zur Kritik aufgefordert. ${ }^{251}$ Eigennutz ist aber erstens scharf zu unterscheiden von Missgunst. Zweitens wird auch nicht behauptet, Altruismus sei keine in der Realität zu findende Verhaltensweise. Eigennutz kann aber als durchschnittliches Verhalten durchaus angenommen werden, vor allem wenn man bedenkt, dass es um die Prognose von Gruppenverhalten geht. Rationalität bedeutet nicht, dass Menschen wie wandelnde Computer handeln. Es wird lediglich unterstellt, dass sie in der Lage sind, ihren Handlungsraum im Großen und Ganzen zu überblicken und die ihnen zu Gebote stehenden Alternativen hinsichtlich ihres Vorteils zu bewerten.

\subsubsection{Verhalten unter unvollständiger Information}

Entgegen dem Eindruck, den Lehrbücher in der Regel vom Homo oeconomicus zu vermitteln pflegen, abstrahiert die moderne Theorie ausdrücklich nicht von unvollständiger Information. Mit dieser Modellerweiterung läuft die Kritik ins Leere, der Homo oeconomicus als stets vollständig informiertes und optimierendes Individuum sei ein realitätsfernes Konstrukt.

Ein Gegenentwurf zum optimierenden Homo oeconomicus ist das Konzept der bounded rationality von Simon ${ }^{252}$. Danach setzt das Individuum vorab ein Anspruchsniveau und sucht solange unter den ihm zugänglichen Alternativen, bis es eine findet, die dem Anspruch mindestens genügt. Findet sich keine akzeptable Alternative, wird das Anspruchsniveau korrigiert und die Suche erneut durchgeführt.

Der ,Satisficer" nach Simon ist ein Spezialfall der oben dargestellten Version des Homo oeconomicus. Die wesentlichen Elemente - die Trennung von

${ }^{249} \mathrm{Vgl}$. etwa die Formalisierung des Ricardianischen Äquivalenztheorems in Barro (1974).

250 Vgl. Rawls (1971), S. 144.

251 Vgl. Arrow (1986), Sen (1997).

252 Vgl. Simon (1955). 
Präferenzen und Restriktionen, die Bewertung der Alternativen, die Entscheidung gemäß dem eigenen Vorteil und damit die Beeinflussbarkeit des Verhaltens durch Anreize - sind auch bei Simon enthalten.

Eingeschränkte Rationalität ist vor allem dann von Bedeutung, wenn Unkenntnis über den Handlungsraum und die Konsequenzen der Handlungsalternativen besteht. Der Einzelne beabsichtigt zwar, vernünftig zu handeln; dies ist ihm aber nur eingeschränkt möglich, da seine Entscheidung von einer Fülle von Handlungsmotiven abhängt und die Kosten der Informationsbeschaffung und Informationsauswertung zu beachten sind. ${ }^{253}$ In solchen Situationen kommt es vor allem darauf an, ,rationale Suchverfahren “254 zu entwickeln. Faustregeln, auf die in der Praxis häufig zurückgegriffen wird, lassen sich mit einem Entscheidungsverhalten, das auf Anspruchsanpassung beruht, gut begründen. Wie leicht man sich mit einer Anspruchserfüllung zufrieden gibt und auf die Suche nach der optimalen Handlungsalternative verzichtet, hängt sehr von den institutionellen Rahmenbedingungen ab. Sorgen die Institutionen für einen starken Konkurrenzdruck, steigen damit tendenziell die Kosten einer suboptimalen Entscheidung. Andererseits spielt eine Rolle, wie gut der Informationsstand des Entscheidenden vorab ist und wie kostspielig die Beschaffung weiterer Informationen ist. Es erscheint plausibel, dass der Anreiz zu optimierendem Verhalten auf polypolistischen Märkten größer ist als für Monopolisten oder gar bei bürokratischen Entscheidungen.

Dem Homo-oeconomicus-Modell ist von Dahrendorf der Homo sociologicus entgegengehalten worden. Dieses Modell beruht auf dem Gedanken, dass menschliches Verhalten weniger von rationalen Entscheidungen als vielmehr von sozialen Normen bestimmt wird. ${ }^{255}$ Der beabsichtigte Gegensatz dieser beiden Konstrukte wird aber im Licht des bisher Gesagten fragwürdig. Auch für den Homo oeconomicus kann es unter unvollständiger Information rational sein, sich an Regeln zu orientieren. Die Regeln werden aber nicht blind angewendet, sondern haben ausdrücklich den Sinn, Informationskosten zu sparen. Insofern sind solche Regeln „kontingent“256, d.h. sie gelten für eine Klasse von Standardsituationen und werden nur dort angewandt. Ändern sich die Umweltbedingungen so, dass die Regeln die entstehenden Situationen nicht mehr abdecken, wird ein rationales Individuum auch die Regeln

\footnotetext{
${ }^{253}$ Vgl. Schneider (1995b), S. 10f. Ihm zufolge bietet der Begriff der beschränkten Rationalität jedoch nur einen anderen Namen für unvollkommene Information des Entscheidenden. Wer rational zu handeln beabsichtigt, tut dies aus seiner Sicht immer; aus der Sicht eines außenstehenden Beobachters mit besserem Wissen jedoch durchweg beschränkt rational. Vgl. hierzu auch Schlicht (1990), S. $710 f$.

${ }^{254}$ Simon (1978), S. 11. Vgl. auch Simon (1979).

255 Vgl. Dahrendorf (1958).

${ }^{256}$ Kirchgässner (2000), S. 33.
} 
anpassen. Solche Regeln kann man als intern bezeichnen. Das Individuum setzt sie sich selbst und prüft vor Anwendung, ob die Regel auf die Entscheidungssituation passt. Doch auch externe Regeln, die an das Individuum von außen herangetragen werden, können im Modell des Homo oeconomicus berücksichtigt werden. Sie stellen dort nichts anderes als eine bestimmte Art von Restriktionen dar, deren Verletzung mit Kosten in Form von Sanktionen verbunden ist. Die Befolgung externer Regeln beruht also auf einem rationalen Abwägen von Kosten und Nutzen der Regelverletzung.

Die Diskussion hat aufgezeigt, dass das Individuum unter unvollständiger Information nicht in der Lage ist, die optimale Handlungsalternative zu finden bzw. als solche zu erkennen. Er muss also „eingeschränkt rational“ handeln, d.h. ein Anspruchsniveau setzen und eine Alternative auswählen, die dem genügt. Diese Bedingungen machen Regeln plausibel, an denen sich das Individuum bei der Suche orientiert. Solche Regeln werden in Kapitel 5 in Form von Rückkopplungsmechanismen verwendet. Das Individuum orientiert sich bei seiner Entscheidungsfindung an seinen Entscheidungen und deren Auswirkungen in der Vergangenheit.

\subsubsection{Arbeitsteilung}

Durch den methodologischen Individualismus rücken diejenigen Probleme in den Vordergrund, die dadurch erst entstehen, dass mehrere Individuen an der Erreichung eines Ziels gemeinsam arbeiten. Arbeitsteilung kann in zwei Formen erfolgen, als Art- oder Mengenteilung. Bei Artteilung wird eine Aufgabe in mehrere Teile aufgespalten, und die Teilaufgaben werden an verschiedene Personen vergeben. Mengenteilung liegt hingegen vor, wenn eine Aufteilung der Gesamtaufgabe nicht möglich ist, aber dennoch mehrere Personen an ihrer Bewältigung mitwirken bzw. mitwirken müssen. Die Vorteile einer solchen Kooperation liegen auf der Hand. Der Produktivitätsgewinn durch Arbeitsteilung wird durch das Beispiel einer Stecknadelmanufaktur von Adam Smith eindrucksvoll beschrieben ${ }^{257}$. Ein Arbeiter, der die Nadeln allein fertigt, schafft 20 Nadeln pro Tag. Wird der Vorgang in 10 Teilaufgaben zerlegt und von 10 auf den jeweiligen Teilvorgang spezialisierten Arbeitern erledigt, steigt der Output auf 4800 Nadeln pro Tag und Arbeiter, eine beeindruckende Steigerung um das 24-fache. Die Gründe für die Produktivitätssteigerung liegen darin, dass unterschiedliche Begabungen ausgenutzt werden können und Lerneffekte durch das kleinere Arbeitsfeld deutlicher sichtbar werden. Personelle Arbeitsteilung macht die Bewältigung von Aufgaben, zu denen

257 Vgl. Smith (1776, 1976). 
ein Einzelner nicht in der Lage wäre, überhaupt erst möglich. So bedarf es mehrerer Arbeiter, einen schweren Gegenstand zu transportieren, den ein einzelner nicht heben kann. ${ }^{258}$

Koordinationsbedarf entsteht nun als Folge der Arbeitsteilung. Durch die Zerlegung der Gesamtaufgabe kann der mit einer Teilaufgabe beauftragte Einzelne den gesamten Vorgang nicht mehr ohne Weiteres überblicken. Dennoch ist es im Interesse der Erfüllung der Gesamtaufgabe notwendig, dass alle Mitwirkenden dazu angehalten werden, ihre Handlungen, d.h. die Erfüllung ihrer Teilaufgaben, auf das übergeordnete Ziel auszurichten.

Der Begriff der Koordination lässt sich also in einer ersten Annäherung fassen als ,die Ausrichtung von Einzelaktivitäten in einem arbeitsteiligen Sys-

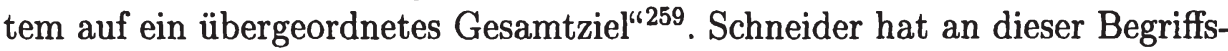
fassung berechtigte Kritik geübt ${ }^{260}$, wobei er das übergeordnete Gesamtziel als gemeinsames Ziel aller Beteiligten auffasst. In einer Hinsicht ist dieses Verständnis zu weit gefasst. Die Abstimmung von Einzelaktivitäten auf ein gemeinsames Ziel hin umfasst auch die Planung und den Vollzug von Handlungen durch den Einzelnen. So verstandene Koordination hat keinen eigenständigen Sinn. Statt dessen muss man die Planung des Einzelnen begrifflich abtrennen durch Betonung der Abstimmung unter mehreren Personen. In einer anderen Hinsicht ist die obige Begriffsfassung zu eng. Die Forderung nach einem gemeinsamen Ziel schließt die Abstimmung über Märkte aus. Nachfrager und Anbieter auf einem Markt verfolgen gerade kein gemeinsames Ziel. Sie suchen trotz gegenläufiger Ziele, nämlich möglichst teuer zu verkaufen bzw. möglichst billig einzukaufen, zu ihrem eigenen Vorteil eine freiwillige Planabstimmung. Ebenso verfolgen Gruppenmitglieder, die sich durch Selbstabstimmung oder Mehrheitsentscheidungen koordinieren wollen, eigene Ziele. Nun könnten Gruppen zwar versuchen, sich in Diskussionen auf gemeinsame Ziele zu einigen. Wie dies in der Praxis konkret aussehen soll, ist aber weitgehend unklar. ${ }^{261}$ Sinnvoller erscheint es, sich darauf zu verständigen, wie verschiedene Ziele durch ein gemeinsames Bündel von Handlungen erreicht werden können. Die Annahme eines gemeinsamen Ziels kann daher nur bedeuten, dass dieses Ziel die Eigeninteressen der Beteiligten überlagert, und ist als Vereinfachung aufzufassen.

\footnotetext{
$\overline{258}$ Vgl. Laux und Liermann (1997), S. 3.

${ }^{259}$ Frese (2000), S. 10.

260 Vgl. Schneider (1997a), S. 449f.

${ }^{261}$ Zur Problematik eines herrschaftsfreien Diskurses und zum Unmöglichkeitstheorem vgl. Schneider (1997a), S. 121ff.
} 
Schneider schlägt deshalb eine andere Definition vor ${ }^{262}$, die das Zusammenwirken mehrerer Personen betont, ohne auf gemeinsame Ziele zu rekurrieren. Koordination besteht danach im Erarbeiten eines gemeinsamen Wirtschaftsplans, wenn noch keine individuellen Wirtschaftspläne existieren, oder in der Abstimmung bereits bestehender Einzelwirtschaftspläne. Darüber hinaus werden Steuerungsregeln benötigt, nach denen sich bestimmt, wer die Umsetzung von Entscheidungen in Handlungen anordnet und wer den Vollzug der Handlungen kontrolliert.

\subsection{Sachliche und personelle Koordination}

Nach Ewert und Wagenhofer können die Ursachen des Koordinationsbedarfs in die beiden Kategorien sachliche und personelle Gründe unterschieden werden. ${ }^{263}$ Sachliche Interdependenzen lassen sich wiederum in vier Ausprägungen unterscheiden. ${ }^{264}$

- Ressourcenverbund. Die Knappheit der zur Verfügung stehenden Ressourcen erfordert eine Abstimmung hinsichtlich der Allokation auf verschiedene Verwendungszwecke. Die Verwendung von Ressourcen für eine Maßnahme begrenzt die Möglichkeiten der Durchführung anderer Maßnahmen. Im Hinblick auf eine optimale Erreichung der Unternehmensziele müssen die einzelnen Maßnahmen also koordiniert werden.

- Erfolgsverbund. Der Erfolgsbeitrag einer Maßnahme kann davon abhängen, welche anderen Maßnahmen noch durchgeführt werden. Die Interdependenzen treten in der Ergebnisfunktion auf.

- Risikoverbund. Sind bei der Entscheidungsfindung unsichere Erwartungen zu berücksichtigen, können die Ergebnisbeiträge von Maßnahmen stochastisch abhängig sein. In Verbindung mit bestimmten Risikopräferenzen ${ }^{265}$ macht die Korrelation der Einzelaktivitäten eine Koordination im Rahmen einer Gesamtbetrachtung notwendig.

- Bewertungsverbund. Eine Interdependenz zwischen verschiedenen Einzelaktivitäten kann schließlich im Präferenzsystem des Entscheidenden

262 Vgl. Schneider (1997a), S. 448f.

${ }^{263}$ Vgl. Ewert und Wagenhofer (2000), S. 446ff.

${ }^{264}$ Vgl. Laux und Liermann (1997), S. $195 \mathrm{ff}$.

${ }^{265}$ Das Entscheidungsverhalten darf nicht risikoneutral sein, und die Risikopräferenz muss am isolierten Risiko des Unternehmens anknüpfen. Vgl. Ewert und Wagenhofer (2000), S. 448 
begründet sein. Die subjektive Bewertung einer bestimmten Maßnahme hängt vom bisherigen Ergebnisniveau und damit implizit von den Ergebnissen anderer Maßnahmen ab.

Die dargestellten Verbundtypen können prinzipiell unabhängig voneinander auftreten. Die Lösung solcher Probleme ist bereits in den obigen Ausführungen angeklungen. Die optimale Koordination beruht auf einer Gesamtbetrachtung, die konzeptionell durch Totalmodelle der Planung durchgeführt wird. Die Interdependenzen der Unternehmensbereiche und damit der Einzelaktivitäten können mit solchen Modellen erfasst und hinsichtlich des Zielsystems optimal koordiniert werden. Totalmodelle sind allerdings nur konzeptionell unproblematisch. Bei der praktischen Umsetzung stoßen sie recht schnell an die Grenzen eines erträglichen Rechenaufwandes. Daher ist man auf die Zerlegung in voneinander unabhängige Teilbereiche mittels der Anwendung von Separationstheoremen und der Verwendung von Heuristiken angewiesen. Hier finden Modelle der hierarchischen Planung Verwendung. ${ }^{266}$

Die bisher beschriebenen sachlichen Gründe für die Existenz von Koordinationsbedarf blenden die Probleme, die aus der Mitwirkung mehrerer Personen entstehen, noch vollständig aus. Das Planungsmodell wird durch die Unternehmensleitung aufgestellt und die Lösung anschließend implementiert. Das Unternehmen agiert damit wie ein Monolith. Dies widerspricht der hier gewählten Konzeption des methodologischen Individualismus. Daher wird in dieser Arbeit davon ausgegangen, dass Individuen auch in einem Unternehmen nach ihren eigenen Interessen handeln und ihre Entscheidungen auf der Basis unterschiedlicher Informationsstände treffen. Wenn ein gemeinsames Ziel unterstellt wird, ist damit implizit angenommen, dass dies den Eigeninteressen der Beteiligten nicht widerspricht.

Die Annahme monolithischen Handelns eines Unternehmens fallen zu lassen bedeutet, die Aufteilung der Entscheidungsbefugnisse auf mehrere Personen mit unterschiedlichem Wissen zu betrachten. Damit eröffnen sich den Entscheidungsträgern Möglichkeiten, ihre Befugnisse im Sinne ihrer eigenen Interessen $\mathrm{zu}$ benutzen, auch wenn dies dem Unternehmen und dessen Anspruchsgruppen zum Nachteil gereicht. Sie müssen also wirksam kontrolliert werden. Die Übertragung von Entscheidungsbefugnissen macht jedoch nur Sinn, wenn damit gleichzeitig auch die Planung für das Teilentscheidungsfeld delegiert wird. Verbliebe die Planung hingegen bei der zentralen Unternehmensführung, könnte diese auch selbst entscheiden. Der Planungsprozess mit

${ }^{266}$ Vgl. Steven (1994) zur Produktionsplanung sowie Homburg und Schneeweiß (1997) zur dezentralen Planung. 
der Beschaffung und Verarbeitung von entscheidungsrelevanten Informationen verschafft den Beauftragten einen Vorsprung an exklusivem Wissen, was eine Kontrolle durch die Zentrale erschwert. Daher muss die Zentrale Sorge tragen, die institutionellen Rahmenbedingungen so auszugestalten, dass die Verfolgung der Unternehmensziele im Interesse der Beauftragten liegt. Solche institutionellen Arrangements werden in der Principal-Agent-Theorie diskutiert. $^{267}$

\subsection{Koordinationsformen}

In der Literatur existiert eine Fülle von Typologien der Koordination, die sich mehr oder weniger voneinander unterscheiden. Grundlegend ist dabei die Unterscheidung in Koordination innerhalb oder außerhalb einer Hierarchie.

Innerhalb einer Hierarchie gibt es Vorgesetzte mit Weisungsbefugnissen und Untergebene; deshalb wird auch von vertikaler Koordination gesprochen. Das Problem besteht darin, die Untergebenen dazu zu veranlassen, die erteilten Weisungen auch tatsächlich im Sinne des Vorgesetzten umzusetzen, zumal die Untergebenen in der Regel einen Informationsvorsprung besitzen, den sie für ihre eigenen Interessen einsetzen können. Oder die Koordination erfolgt außerhalb einer Hierarchie unter gleichberechtigten Individuen, die gleichwohl nicht den gleichen Einfluß besitzen müssen. Wegen der Gleichberechtigung bezeichnet man heterarchische Koordinationsformen auch als horizontale Koordination.

Ferner kann man Koordinationsformen hinsichtlich der Existenz eines gemeinsamen Zieles aller Beteiligten klassifizieren. Gibt es ein solches Ziel, reduziert sich der Koordinationsbedarf auf sachliche Koordination. Ohne gemeinsames Ziel tritt die Notwendigkeit persönlicher Koordination hinzu.

Es wird sich jedoch zeigen, dass diese Klassifikationsmerkmale keineswegs in allen Typologien wiederzufinden sind. Einige Autoren benutzen auch andere Merkmale, andere wiederum verzichten völlig auf unterscheidende Merkmale und stellen die Koordinationsformen einfach nebeneinander. Im nächsten Abschnitt werden in der Literatur vorgestellte Typologien kurz dargestellt und kritisch gewürdigt.

$\overline{267}$ Vgl. Jensen und Meckling (1976), Alchian und Demsetz (1972), Ross (1973) und für einen Überblick z.B. Bamberg und Spremann (1987) sowie Elschen (1991). 


\subsubsection{Koordinationstypologien in der Literatur}

Schäffer orientiert sich explizit an den oben dargestellten Klassifikationsmerkmalen. Er entwirft auf der Grundlage der Merkmale Zielausrichtung und Koordinationsrichtung einen typologischen Kasten, der in Abbildung 4.1 dargestellt ist. ${ }^{268}$ Sein Ziel ist speziell die Einordnung des in der Literatur uneinheitlich gehandhabten Begriffes Selbstabstimmung. Dabei lehnt er sich an die Dichotomie Organisation und spontane Ordnung ${ }^{269}$ nach von Hayek an und bezeichnet vertikale Koordination mit gemeinsamem Zweck als Organisation, und horizontale Koordination ohne gemeinsamen Zweck als spontane Ordnung. Im ersten Fall finden die Koordinationsmechanismen Pläne und Anordnungen Verwendung, im zweiten Fall dienen Preismechanismus bzw. Verhandlungen zur Koordination. Damit bleiben in seinem Schema zunächst noch zwei Felder frei. Horizontale Koordination bei gemeinsamem Zweck wird nun von Schäffer als Selbstabstimmung bezeichnet. Das Beispiel „two people in a canoe" übernimmt er von Mintzberg. ${ }^{270}$ Den Fall vertikaler Koordination ohne gemeinsamen Zweck versteht er als „Koordination durch Zwang beispielsweise in einer Sklavenhaltergesellschaft oder Gefangenenlagern" ${ }^{\text {271. }}$. Die Anwendung von Zwang ist für ihn jedoch keine zulässige Koordinationsform.

Kieser und Kubicek untersuchen ausschließlich Organisationen, bei denen ein gemeinsames Ziel vorausgesetzt werden kann. ${ }^{272}$ Marktkoordination spielt deshalb bei ihnen keine Rolle. Die Unterscheidung nach vertikaler und horizontaler Koordination lässt sich implizit finden.

Wie Abbildung 4.2 zeigt, unterscheiden Kieser und Kubicek danach, ob die Mechanismen auf einer unmittelbaren persönlichen Kommunikation beruhen (personenorientierte Instrumente) oder sich zu einer Institution verselbstständigen (technokratische Instrumente), d.h. Gliederungskriterium sind die Medien der Koordination. Personenorientierte Koordination kann wiederum in Form persönlicher Weisungen erfolgen, wenn eine Personenhierarchie mit entsprechenden Rechten der übergeordneten Ebenen besteht, oder durch Selbstabstimmung, bei der die Koordination durch Eigeninitiative der Gruppe ohne Hierarchie erfolgt. Von unverbindlichem Informationsaustausch wird Selbstabstimmung dadurch abgegrenzt, dass die Gruppenentscheidung offiziell vorgesehen und für alle Gruppenmitglieder verbindlich sein muss. Bei

\footnotetext{
268 Vgl. Schäffer (1996).

${ }^{269} \mathrm{Vgl}$. von Hayek $(1969,1994)$.

270 Vgl. Mintzberg (1979), S. 3.

${ }^{271}$ Schäffer (1996), S. 1096, H.i.O.

272 Vgl. Kieser und Kubicek (1992), S. 95ff.
} 


\begin{tabular}{|c|c|c|}
\hline & $\begin{array}{c}\text { Vertikale } \\
\text { Koordination }\end{array}$ & $\begin{array}{c}\text { Horizontale } \\
\text { Koordination }\end{array}$ \\
\hline $\begin{array}{c}\text { Gemeinsamer } \\
\text { Zweck }\end{array}$ & $\begin{array}{c}\text { Pläne und } \\
\text { Anordnungen }\end{array}$ & Selbstabstimmung \\
\hline $\begin{array}{c}\text { Kein } \\
\text { gemeinsamer } \\
\text { Zweck }\end{array}$ & - & $\begin{array}{c}\text { Preismechanismus } \\
\text { bzw. } \\
\text { Verhandlungen }\end{array}$ \\
\hline
\end{tabular}

Quelle: Schäffer (1996), S. 1099

Abbildung 4.1: Koordinationsinstrumente nach Schäffer

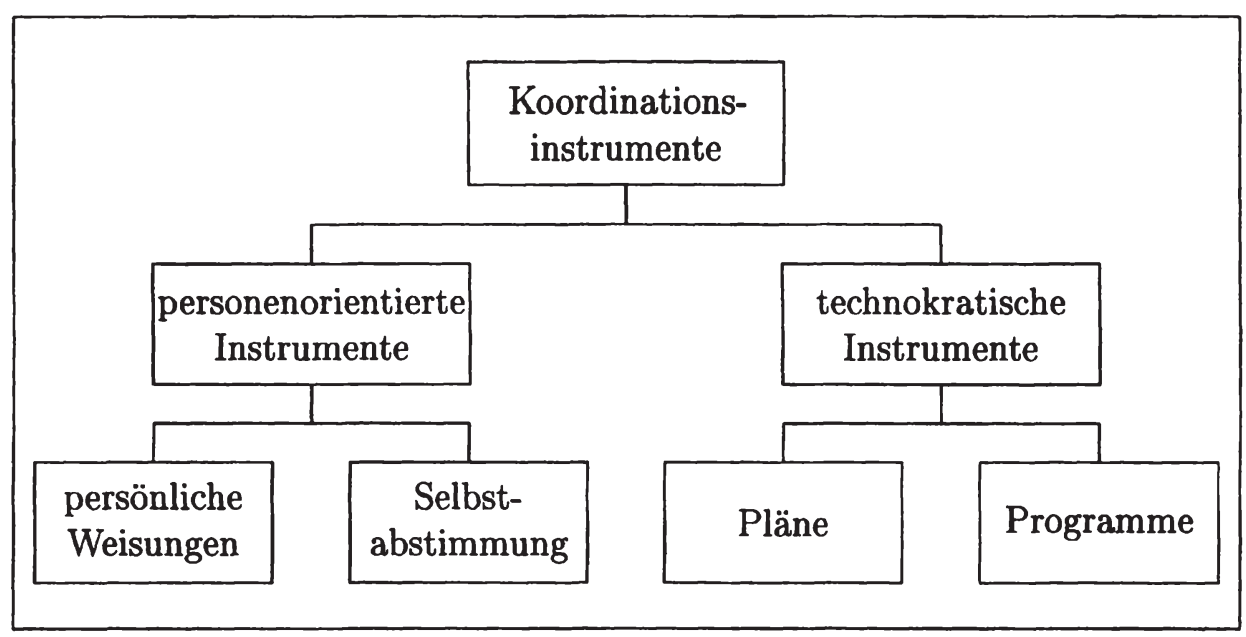

Abbildung 4.2: Koordinationsinstrumente nach Kieser und Kubicek

technokratischer Koordination ist der Urheber der verbindlichen Festlegungen meist nicht erkennbar und auch nicht von Interesse. Solche unpersönliche Koordination erfolgt durch Programme oder Pläne. Programme sind festgeschriebene Verfahrensabläufe. Die möglicherweise auftretenden Probleme werden definiert und Verfahren zu ihrer Lösung vorgegeben. Damit verringern sie den Weisungsbedarf, denn die Koordination wird in den definierten Problemstellungen automatisiert. Unter Plänen werden bestimmte Vorgaben 
für ausführende Stellen verstanden, die deren Aktivitäten koordinieren. Sie kommen im Rahmen eines institutionalisierten Planungsprozesses zustande, wobei sie im Gegensatz zu Programmen auf bestimmte Perioden begrenzt sind und sich hinsichtlich des Planinhaltes von Periode zu Periode ändern können. Damit sind sie wesentlich flexibler als Programme, die vor unvorhergesehenen Problemstellungen versagen.

Laux und Liermann gehen von der Dichotomie von Markt und Unternehmung aus. ${ }^{273}$ Die Begründung für die gleichzeitige Existenz beider grundlegender Koordinationsformen übernehmen sie von Coase. ${ }^{274} \mathrm{Im}$ Weiteren beschäftigen sie sich nur noch mit der Unternehmung oder Organisation, für die sie wiederum drei Unterfälle der Koordination identifizieren ${ }^{275}$, die sie jedoch ausdrücklich nicht als einander ausschließende Alternativen verstanden wissen wollen ${ }^{276}$, nämlich Selbstabstimmung, Gruppenabstimmung und Hierarchie. Selbstabstimmung liegt vor, wenn jeder Beteiligte eigenständig über seine Tätigkeiten entscheidet und die Abstimmung im Ermessen der Betroffenen liegt. In der Praxis kann darunter nur eine sukzessive wechselseitige Abstimmung verstanden werden, die jedoch bei großer Anzahl an Beteiligten und starken Interdependenzen der Einzelaufgaben regelmäßig zu extrem hohem Koordinationsaufwand führt. Trotz ausdrücklicher Bezugnahme der Autoren auf Kieser und Kubicek (1992) unterscheiden sich die Begriffsverwendungen. Was Kieser und Kubicek unter Selbstabstimmung verstehen, korrespondiert eher mit der Gruppenabstimmung nach Laux und Liermann. Danach entscheiden die Gruppenmitglieder mittels einer Abstimmungsregel über die Aufteilung der Tätigkeiten. Die Entscheidung ist für jedes Gruppenmitglied verbindlich. ${ }^{277}$ Bei der Hierarchie schließlich sind einige Individuen, sogenannte Instanzen, mit Entscheidungs- und Weisungsrechten ausgestattet. Ihre Aufgabe ist ausdrücklich die Koordination der übrigen Mitglieder der Organisation, die den Weisungen Folge zu leisten haben. Abbildung 4.3 zeigt die Koordinationsformen noch einmal in der Übersicht.

Der Ansatz von Thompson ist eher soziologischer denn ökonomischer Natur. Die institutionellen Rahmenbedingungen einer Organisation stehen im Vordergrund der Analyse, nicht die individuelle Entscheidungsfindung. ${ }^{278} \mathrm{Er}$ unterscheidet drei Koordinationsformen ${ }^{279}$ : Anweisung, Gruppenidentifikati-

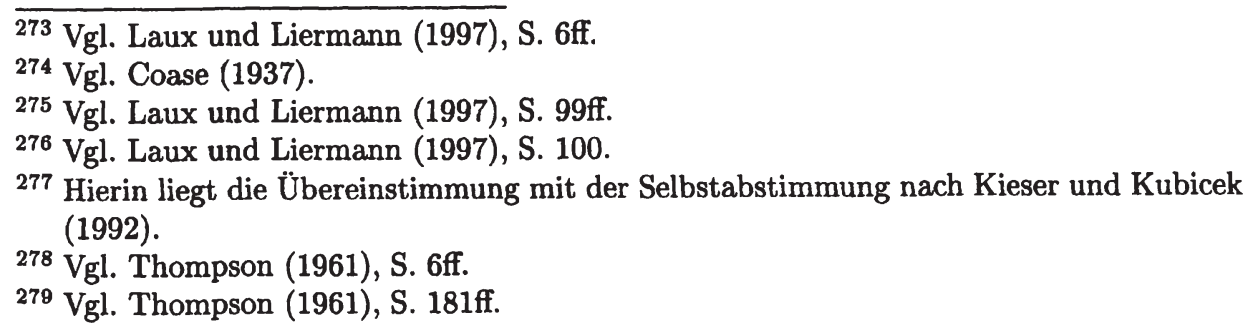




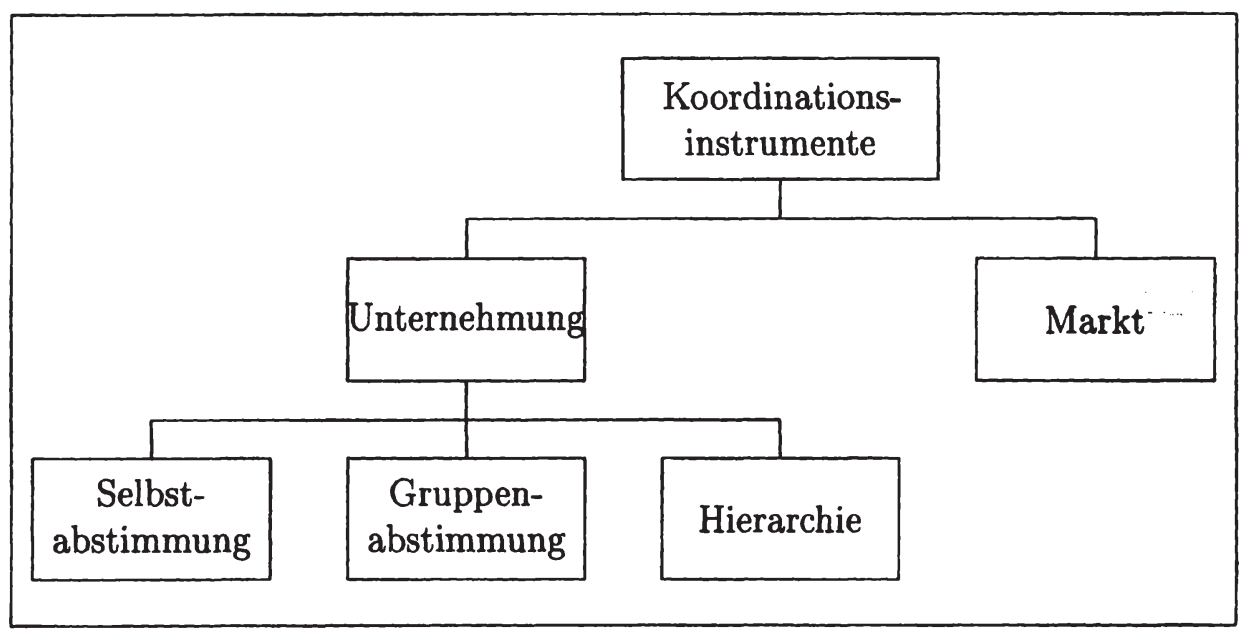

Abbildung 4.3: Koordinationsinstrumente nach Laux und Liermann

on und anerkannte gegenseitige Abhängigkeit ${ }^{280}$. Unter Anweisung sind alle Instrumente zusammengefassßt, die auf Über- und Unterordnung basieren, also Instrumente vertikaler Koordination. Die Problematik dieser Koordinationsform liegt zunächst in ihren Anwendungsvoraussetzungen. Die Anweisungen müssen verständlich und konsistent sein; ihre Ausführung muss durch die Autorität der Vorgesetzten oder durch ein System von Belohnungen und Sanktionen sichergestellt werden. Des Weiteren ist von Nachteil, dass ein autoritäres Koordinationssystem die Motivation der Untergebenen in der Regel konterkariert. Die Koordination durch Gruppenidentifikation versucht auszunutzen, dass informelle Gruppen sich durch gemeinsame Werte und Wahrnehmungen in großem Ausmaß selbst koordinieren, indem sie dies auf formale Gruppen innerhalb von Unternehmen übertragen. Dieser Ansatz betont die Notwendigkeit, dass Individuen von sich aus zur Kooperation bereit sein müssen, um erfolgreich koordiniert werden zu können, und stellt die Wirksamkeit individueller Anreize in Abrede. Koordination auf Basis anerkannter gegenseitiger Abhängigkeit betont die Effektivität von Gruppenentscheidungen, in die das Wissen verschiedener Spezialisten einfließen kann. Sie läuft letztlich auf ein Anreizsystem hinaus, das denjenigen begünstigt, der seine Tätigkeit in den Dienst der Gruppenziele stellt. Wie die genannten Formen der Koordination in der Praxis ablaufen sollen, bleibt unklar. Es handelt sich auch

280 „recognized mutual interdependence“, Thompson (1961), S. 187. Die Übersetzung "gegenseitig anerkannter Abhängigkeiten" von Schäffer (1996), S. 1096, ist nicht korrekt. 
weniger um konkrete Koordinationsinstrumente als vielmehr um Koordinationsformen ${ }^{281}$.

Schneider unterscheidet in einem ersten Schritt nach Koordination außerhalb und innerhalb einer Hierarchie. ${ }^{282}$ Im ersten Fall erfolgt die Koordination ohne Übertragung von Entscheidungsbefugnissen auf Beauftragte. Sie findet entweder in Entscheidungsgruppen formal gleichberechtigter Personen oder in Märkten statt. Die beteiligten Personen brauchen gleichwohl nicht gleich mächtig zu sein. Der zweite Fall geschieht mit Beauftragung, durch die die Hierarchie entsteht. Hier werden drei Instrumente unterschieden. Koordination durch Diktat erfolgt mittels Befehlen, die von den Untergebenen umgesetzt werden, entweder um Sanktionen zu entgehen, oder auch aufgrund von Einsicht in die Notwendigkeit. Bei Delegation werden Aufgaben an Mitglieder nachgeordneter Stufen einer Hierarchie zugewiesen, die diese im Rahmen eines verbindlichen, aber groben Gesamtplans zu erfüllen haben. An der Erstellung des Gesamtplans sind die unteren Hierarchiestufen beteiligt. Innerhalb dieses Plans können sie einen begrenzten Handlungsspielraum nutzen. Dezentralisation baut auf finanzielle Rahmenziele wie Bereichsgewinn oder strategische Nebenbedingungen wie Marktanteil als Vorgabe. Wie dies in Detailpläne umgesetzt wird, bleibt vollständig den nachgeordneten Hierarchiestufen überlassen. Abbildung 4.4 zeigt die Typologie nach Schneider in der Übersicht.

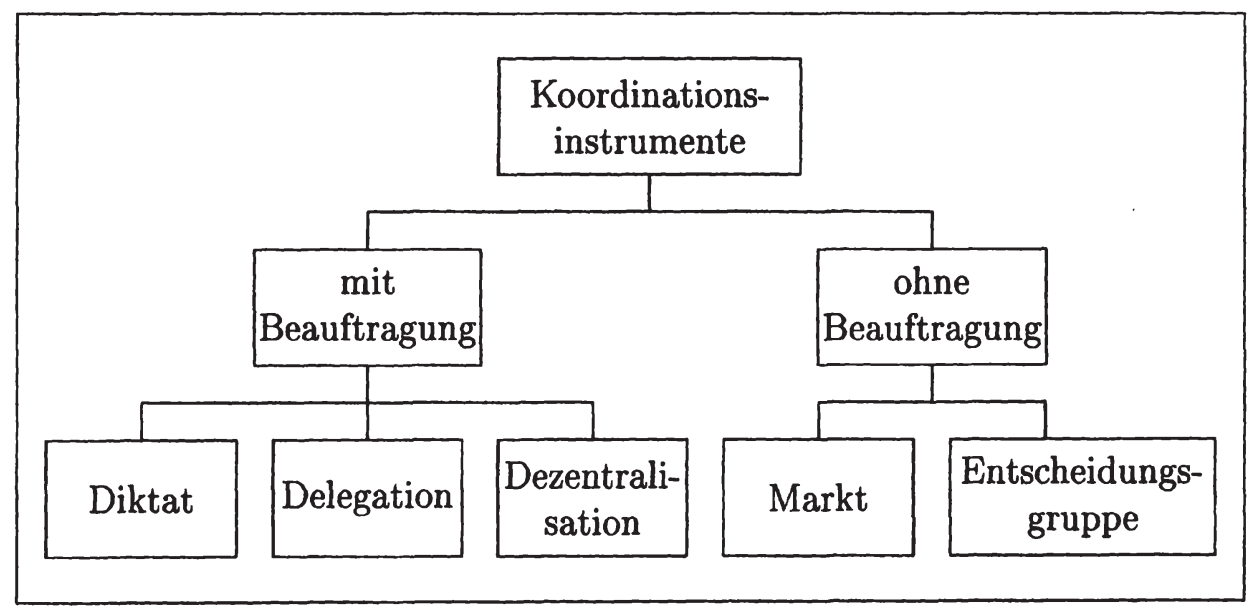

Abbildung 4.4: Koordinationsinstrumente nach Schneider

281 Thompson (1961), S. 187, spricht von einer "basis for coordination".

282 Vgl. Schneider (1997a), S. 452. 


\subsubsection{Kritische Würdigung}

Die Diskussion des letzten Abschnitts hat vor allem gezeigt, dass keine einheitliche Auffassung darüber existiert, nach welchen Klassifikationsmerkmalen Formen der Koordination voneinander abzugrenzen sind. Der Grund hierfür ist, dass die Fülle möglicher Koordinationsformen ein Kontinuum darstellt, d.h. jedes Klassifikationsmerkmal besitzt letztendlich unendlich viele Ausprägungen. Versucht man eine Abgrenzung, so steckt darin notwendig ein gewisses Maß Willkür mit der Folge mangelnder Trennschärfe. Einen Ausweg bietet eine Problemorientierung der Typologie: Jede Typologie muss sinnvollerweise von einer konkreten Problemstellung ausgehen, die der einzige Maßstab der Beurteilung ist, d.h. jede Kritik an einer Typologie muss sich auf den damit verfolgten Analysezweck beziehen.

Weitgehende Einigkeit herrscht darüber, dass zwischen Koordination innerhalb und außerhalb einer Hierarchie bzw. vertikaler und horizontaler Koordination zu unterscheiden ist. Diese Einteilung findet sich, mehr oder weniger ausdrücklich, in jedem Klassifikationsschema wieder. Indes lässt selbst ein solch weitgehend anerkanntes und plausibles Klassifikationsmerkmal keine eindeutige Trennung zu. Bei formaler Gleichberechtigung der beteiligten Personen, also außerhalb einer Hierarchie, sind eine Fülle ökonomischer und außerökonomischer Gründe denkbar, die de facto eine Art von Hierarchie etablieren. So können eine Monopolstellung, langfristige Vertragsbeziehungen oder auch verfügbare Drohungen und Verhandlungsgeschick einem der Beteiligten eine Überlegenheitsposition sichern. Auf der anderen Seite kann eine formale Weisungsbefugnis innerhalb einer Hierarchie durch Informationsvorsprünge oder Überzeugungskraft des Untergebenen oder freundschaftliche Beziehungen zwischen Vorgesetztem und Untergebenem aufgeweicht werden. Letztlich findet man ein Kontinuum möglicher Machtverhältnisse zwischen tatsächlich durchsetzbarer, uneingeschränkter Weisungsbefugnis und tatsächlicher Gleichberechtigung.

Und selbst wenn man eine Trennlinie zwischen Hierarchie und Heterarchie gezogen hat, gibt es innerhalb dieser Kategorien keine eindeutigen Abgrenzungen verschiedener Koordinationsformen. So versucht Schneider die s.E. unklaren Überlappungen und Abgrenzungen zwischen den Begriffen Dezentralisation und Delegation zu vermeiden. ${ }^{283}$ Doch auch ihm gelingt keine Trennschärfe mit der Gleichsetzung von Delegation und Kostenverantwortung bei Planvorgaben bzw. Dezentralisation und Gewinnverantwortung bei selbstgesetzten Detailplänen. Zwischen diesen beiden Formen sind wiederum

$\overline{283}$ Vgl. Schneider (1997a), S. 456 und Fn. 25. 
Zwischenformen mit unterschiedlicher Strenge der Planvorgaben denkbar, die eine eindeutige Abgrenzung unmöglich bzw. willkürlich machen.

Der in dieser Arbeit verfolgte Analysezweck ist nachzuweisen, unter welchen Bedingungen das Bemühen um Koordination mehrerer Individuen zu chaotischen Zeitpfaden führen kann. Dafür kommt es nicht so sehr darauf an, eine Typologie zu entwickeln, die frei von Zweifelsfällen und willkürlichen Einteilungen ist. Die obige Diskussion sollte auch gezeigt haben, dass ein solches Unterfangen fruchtlos wäre. Eine Typologie kann hier lediglich als „Hinterkopfschema" zur ungefähren Einordnung der später zu untersuchenden Szenarien dienen, nicht aber im Sinne einer deskriptiven Theorienbildung selbst Analysezweck sein.

Die Darstellung chaotischer Koordinationsmodelle im folgenden Kapitel orientiert sich an dem Schema von Schäffer, da die vorgeschlagenen Kriterien Koordinationsrichtung und Zweckverhältnis für die gewählten Analyseinstrumente brauchbar erscheinen. Zu jedem der drei besetzten Felder der Abbildung 4.1 wird ein Modell entworfen. 


\section{Kapitel 5}

\section{Koordination und Chaos}

Die Suche nach chaotischen Verhaltensmustern bei Koordinationsversuchen erfordert mathematische Modelle der Koordination. Die Spieltheorie bietet ein geeignetes Instrumentarium zur formalen Analyse von Koordinationssituationen. Zunächst werden in Abschnitt 5.1.1 die wichtigsten Grundbegriffe und Definitionen der Spieltheorie eingeführt. Zwei grundlegende Konzepte werden in Abschnitt 5.1.2 unterschieden: die klassische nichtkooperative Spieltheorie und die evolutionäre Spieltheorie. ${ }^{284}$ Die nichtkooperative Spieltheorie beruht auf dem Konzept der Rationalität, wie es für den homo oeconomicus unterstellt wird. Die Leistungen, aber auch die Grenzen dieses Konzepts werden in Abschnitt 5.1.3 skizziert. Abschnitt 5.1.4 beinhaltet ein konvergentes Koordinationsmodell auf der Grundlage der evolutionären Spieltheorie.

Beide Ansätze haben das Ziel, ein eindeutiges Ergebnis zu generieren, um an die Spieler Handlungsempfehlungen geben zu können. Damit liegt der Fokus bei der Modellkonstruktion auf stabilen Gleichgewichten. Abschnitt 5.2 hingegen untersucht die Rahmenbedingungen, unter denen man keine Konvergenz zu einem Gleichgewicht, sondern chaotische Zeitpfade finden kann.

\subsection{Koordination in der Spieltheorie}

Allgemein gesprochen sind strategische Interaktionssituationen der Gegenstand der Spieltheorie. Das Spielergebnis wird von den Handlungen mehrerer Individuen, die sich dieser Interdependenz bei ihren Entscheidungen bewusst

$\overline{284}$ Die kooperative Spieltheorie wird in der vorliegenden Arbeit nicht verwendet. 
sind, gemeinsam bestimmt. ${ }^{285}$ Jedem Spieler wird vom Spielergebnis eine so genannte Auszahlung zugeteilt, die auch negativ sein kann und eher allgemein als Nutzenmaß denn speziell als Geldwert aufzufassen ist.

Das Schachspiel ist z.B. ein Spiel im Sinne der Spieltheorie. Die Handlungen der Spieler sind die Züge, die in Übereinstimmung mit den allgemein bekannten Regeln auszuführen sind. Das Spielergebnis besteht im Gewinn oder Verlust einer Partie. Die Regeln können durchaus auch so gestaltet sein, dass unvorhersehbare Ereignisse möglich sind und das Spielergebnis beeinflussen. Die Situation gleicht dann eher einem Karten- oder Würfelspiel. Zufällige, unvorhersehbare Ereignisse werden in ein Spiel einbezogen, indem ein Spieler, der Natur genannt wird, Aktionen gemäß einer vorgegebenen Wahrscheinlichkeitsverteilung auswählt, beispielsweise die Kartenverteilung beim Skat. Die klassische Entscheidungstheorie ${ }^{286}$ kann so als Teilgebiet der Spieltheorie aufgefasst werden, nämlich als die Analyse von Spielen eines Spielers gegen die Natur. In der Regel wird unterstellt, die Spieler handelten durchweg rational, d.h. sie wählen ihre Aktionen so, dass ihre Auszahlung maximal wird. Es ist allerdings nicht nötig, die Spieler ausschließlich als Maximierer zu modellieren, was in etwa dem älteren, strengeren Modell des homo oeconomicus entspräche. Man kann auch irrationales, satisfizierendes oder Regeln folgendes Verhalten modellieren, indem man die Auswahlregeln, mit denen die Spieler die durchzuführenden Aktionen bestimmen, entsprechend ändert. Der Spieler Natur ist das naheliegendste Beispiel für irrationales Verhalten. Die evolutionäre Spieltheorie befasst sich in ihren biologischen Anwendungen ausschließlich mit nicht vernunftbegabten Spielern. ${ }^{287}$

\subsubsection{Strategische Form und Bimatrix-Form}

Ein Spiel in strategischer Form ${ }^{288}$ wird beschrieben durch das Tripel $\Gamma=$ $(I, S, \mathbf{p})$. Es enthält $n$ Spieler, die in der Indexmenge $I=\{1, \ldots, n\} \subset \mathbb{N}$ zusammengefasst werden. Jeder Spieler $i$ verfügt über eine endliche Strategienmenge $S_{i}=\left\{1, \ldots, m_{i}\right\} \subset \mathbb{N}$. Eine Strategie ist ein vollständiger Plan für das gesamte Spiel, der einem Spieler für jede denkbare Spielsituation sagt,

285 Vgl. Holler und Illing (1993), S. 1. Die tatsächliche Ausführung einer Handlung spielt in der Theorie keine Rolle, so dass Handlung und Entscheidung miteinander identifiziert werden. Vgl. Rasmusen (1994), S. 10.

${ }^{286}$ Vgl. etwa Mag (1990), Bamberg und Coenenberg (1996) oder Luce und Raiffa (1967).

287 Vgl. Maynard Smith (1982) oder Weibull (1995).

${ }^{288}$ Vgl. Hart (1992), S. 26 und zur extensiven Form S. 22. Die strategische Form wird mitunter auch als Normalform bezeichnet. 
welche Handlung er zu wählen hat. ${ }^{289}$ Die Strategien werden wie die Spieler durchnummeriert. Der Vektor $\mathbf{s}=\left(s_{1} \ldots s_{n}\right) \in \mathbb{N}^{n}$ enthält als Elemente die von jedem der $n$ Spieler gewählte Strategie und heißt Strategienprofil. Die Menge aller Strategienprofile wird Strategienraum genannt und ist das kartesische Produkt aller Strategienmengen $S=\times_{i \in I} S_{i} \subset \mathbb{N}^{n}$. Jeder Spieler $i$ erhält als Spielergebnis eine Auszahlung, die durch die Auszahlungsfunktion

$$
\forall i \in I: \quad p_{i}: S \rightarrow \mathbb{R}, \quad \mathbf{s} \mapsto p_{i}(\mathbf{s})
$$

bestimmt wird. Die Auszahlungen hängen also vom gesamten Strategienprofil ab, d.h. auch von den Strategien der anderen Spieler. Die Auszahlungen werden in Erwartungsnutzeneinheiten gemessen. Nicht-monetäre Ziele und die Risikoeinstellung lassen sich so in die Spielregeln aufnehmen. Die individuellen Auszahlungen $p_{i}$ werden zu einem Auszahlungsprofil $\mathbf{p}=\left(p_{1} \ldots p_{n}\right)$ zusammengefasst, d.h. es gibt eine Auszahlungsfunktion

$$
\mathbf{p}: S \rightarrow \mathbb{R}^{n}, \quad \mathbf{s} \mapsto \mathbf{p}(\mathbf{s}) .
$$

Das Tripel $\Gamma=(I, S, \mathbf{p})$ enthält die Spielregeln implizit, da vorausgesetzt wird, dass mit den Regeln unverträgliche Verhaltensweisen und Ergebnisse unmöglich sind. ${ }^{290}$

Der Strategienraum $S$ enthält streng genommen nur sogenannte reine Strategien. Jeder Spieler $i$ muss sich für genau eine Strategie $s_{i} \in S_{i}$ entscheiden. Statt dessen kann man zulassen, dass ein Spieler eine Wahrscheinlichkeitsverteilung über seine Strategienmenge wählt. Die Wahrscheinlichkeitsverteilung wird bei endlichen Strategienmengen beschrieben durch einen Vektor

$$
\mathbf{x}_{i}=\left(x_{i 1} \ldots x_{i m_{i}}\right) \in \Delta_{i}=\left\{\mathbf{x}_{i}: \forall s_{i} \in S_{i}\left(x_{i s_{i}} \geq 0 \wedge \sum_{s_{i} \in S_{i}} x_{i s_{i}}=1\right)\right\},
$$

der jeder Strategie $s_{i} \in S_{i}$ eine Wahrscheinlichkeit $x_{i s_{i}} \in \Delta_{i}$ zuordnet, wobei $\Delta_{i}$ der $m_{i}$-dimensionale Einheitssimplex ist. Die reinen Strategien $s_{i} \in S_{i}$ sind darin als Spezialfall $\mathbf{x}_{i}=\mathbf{e}_{i}^{s_{i}}$ enthalten. ${ }^{291}$

Der gemischte Strategienraum wird bezeichnet mit $\Theta=\times_{i \in I} \Delta_{i}$, ein $\mathbf{x}=$ $\left(\mathbf{x}_{1} \ldots \mathbf{x}_{n}\right) \in \Theta$ heißt gemischtes Strategienprofil. Zu gemischten Strategien

289 Vgl. Rieck (1993), S. 35.

290 Wenn man die Möglichkeit einer „Regelverletzung“ einräumen will, muss man die Spielregeln entsprechend ändern, z.B. indem man eine „verbotene" Strategie mit einer Sanktion, also einer Auszahlungsminderung, versieht.

$291 \mathbf{e}_{i}^{s_{i}}$ bezeichnet den $m_{i}$-dimensionalen Einheitsvektor mit der Eins an $s_{i}$-ter Stelle. 
gehören die Auszahlungsfunktionen

$$
\forall i \in I: \quad u_{i}: \Theta \rightarrow \mathbb{R}, \quad \mathbf{x} \mapsto u_{i}(\mathbf{x})=\sum_{s \in S} p_{i}(\mathbf{s}) \prod_{i \in I} x_{i s_{i}} .
$$

Das Produkt $\prod_{i \in I} x_{i s_{i}}$ ist die Wahrscheinlichkeit, dass ein bestimmtes Strategienprofil s gespielt wird. Es wird damit implizit unterstellt, dass die Wahlen der tatsächlich gespielten Strategien der einzelnen Spieler voneinander stochastisch unabhängig sind. Auch die gemischten Auszahlungsfunktionen werdên zü èinem Auszahlungsprofil zusammengefasst:

$$
\mathbf{u}: S \rightarrow \mathbb{R}^{n}, \mathbf{x} \mapsto \mathbf{u}(\mathbf{x})=\left(\mathbf{u}_{1}(\mathbf{x}) \ldots \mathbf{u}_{n}(\mathbf{x})\right) .
$$

Damit ist die gemischte Erweiterung $\Gamma=(I, \Theta, \mathbf{u})$ vollständig erläutert.

Zwei-Personen-Spiele mit $I=\{1,2\}$ lassen sich übersichtlich in der Bimatrix-Form darstellen. Die beiden Matrizen $\mathbf{A}=\left(a_{s_{1} s_{2}}\right)$ und $\mathbf{B}=\left(b_{s_{1} s_{2}}\right)$ sind $m_{1} \times m_{2}$-dimensional und enthalten als Einträge die Auszahlungen $a_{s_{1} s_{2}}=p_{1}\left(s_{1}, s_{2}\right)$ bzw. $b_{s_{1} s_{2}}=p_{2}\left(s_{1}, s_{2}\right)$. Die Auszahlungen bei gemischten Strategien lassen sich daraus leicht berechnen:

$$
u_{1}(\mathbf{x})=\mathbf{x}_{1}^{\prime} \mathbf{A} \mathbf{x}_{2} \quad \text { und } \quad u_{2}(\mathbf{x})=\mathbf{x}_{2}^{\prime} \mathbf{B}^{\prime} \mathbf{x}_{1}
$$

Zwei Spezialfälle der Auszahlungsfunktionen werden im Weiteren eine besondere Rolle spielen, die Symmetrie und die Doppelsymmetrie ${ }^{292}$ :

\section{Definition 5.1}

Ein Spiel $\Gamma=(I, S, \mathbf{p})$ heißt symmetrisches Zwei-Personen-Spiel, wenn $\mathbf{A}^{\prime}=$ B. Ein symmetrisches Zwei-Personen-Spiel ist doppelsymmetrisch, wenn $\mathbf{A}^{\prime}=$ A.

In symmetrischen Spielen gilt $\Delta_{1}=\Delta_{2}=\Delta$ und $\Theta=\Delta^{2}$. Außerdem stimmen die Auszahlungsfunktionen überein: $u_{1}(\mathbf{x})=u_{2}(\mathbf{x})=u(\mathbf{x})$.

\subsubsection{Lösungskonzepte}

Jeder Strategienvektor $\mathbf{x} \in \Theta$ stellt ein mögliches Spielergebnis dar. Ein Konzept, das aus der Menge aller möglichen Ergebnisse die sinnvollen und damit als tatsächliches Spielergebnis vorhersagbaren aussondert, heißt Lösungskonzept $\mathcal{L}$. Formal ist es eine Korrespondenz der Menge aller Spiele $G$, die durch 292 Vgl. Weibull (1995), S. 25f. 
das Tripel $\Gamma=(I, \Theta, \mathbf{u})$ beschrieben werden können, in die Potenzmenge des Strategienraums:

$$
\mathcal{L}: G \rightarrow \wp(\Theta)
$$

In diesem Abschnitt werden zwei Lösungskonzepte vorgestellt, die für ihren Zweig der Spieltheorie grundlegende Bedeutung haben, nämlich das Nash-Gleichgewicht NE und das Gleichgewicht in evolutionsstabilen Strategien ESS.

\subsubsection{Klassische Spieltheorie und Nash-Konzept}

Das Nash-Gleichgewicht basiert auf einer intuitiv einleuchtenden Vorstellung von Rationalität. Ein Strategienvektor ist dann ein sinnvolles Spielergebnis, wenn es sich für keinen Spieler lohnt, einseitig davon abzuweichen, oder mit anderen Worten, wenn jeder Spieler die beste Antwort auf die Strategien der anderen Spieler wählt. ${ }^{293}$ Die beste Antwort $\beta_{i}$ des Spielers $i$ auf das Strategienprofil $\mathbf{y} \in \Theta$ ist die Korrespondenz ${ }^{294}$

$$
\beta_{i}: \Theta \rightarrow \Delta_{i}, \quad \mathbf{y} \mapsto\left\{\mathbf{x}_{i}: \forall z_{i} \in \Delta_{i}\left(u_{i}\left(x_{i}, \mathbf{y}_{-i}\right) \geq u_{i}\left(z_{i}, \mathbf{y}_{-i}\right)\right)\right\} .
$$

Die besten Antworten aller Spieler auf ein gegebenes Strategienprofil y werden zusammengefasst in

$$
\beta: \Theta \rightarrow \Theta, \quad \mathbf{y} \mapsto x_{i \in I} \beta_{i}(\mathbf{y})
$$

\section{Definition 5.2}

Ein Strategienvektor $\mathbf{x} \in \Theta$ heißt Nash-Gleichgewicht, wenn gilt:

$$
\mathbf{x} \in \beta(\mathbf{x})
$$

Die Menge aller Nash-Gleichgewichte heißt $\Theta^{N E} \subset \Theta$. Ein Nash-Gleichgewicht heißt strikt, wenn

$$
\beta(\mathbf{x})=\{\mathbf{x}\}
$$

Satz 5.1

Für jedes Spiel $\Gamma=(I, \Theta, \mathbf{u})$ gilt $\Theta^{N E} \neq \emptyset$.

293 Der formale Apparat ist entnommen aus Weibull (1995), S. 12ff.

${ }^{294}$ Der Vektor $\mathbf{y}_{-i}$ entsteht aus dem Vektor $\mathbf{y}$ durch Weglassen des $i$-ten Elements, $\mathbf{y}_{-i}=$ $\left(y_{1} \ldots y_{i-1} y_{i+1} \ldots y_{n}\right)$. 
Beweis. Ein Nash-Gleichgewicht ist ein Fixpunkt der Korrespondenz $\beta: \Theta \rightarrow \Theta . \Theta$ ist nicht leer, konvex und kompakt. $\beta$ ist oberhalbstetig, und das Bild $\beta(\mathbf{x})$ ist ebenfalls nicht leer, konvex und kompakt. Damit sind die Voraussetzungen von Kakutanis Fixpunktsatz ${ }^{295}$ erfüllt und es existiert ein $\mathbf{x} \in \Theta$ mit $\mathbf{x} \in \beta(\mathbf{x})$.

In einem symmetrischen Zwei-Personen-Spiel wird ein NashGleichgewicht $(\mathbf{x}, \mathbf{y})$ symmetrisch genannt, wenn $\mathbf{x}=\mathbf{y}$ ist. Die Menge aller Strategien, die symmetrische Nash-Gleichgewichte bilden, wird bezeichnet mit

$$
\Delta^{N E}=\left\{x \in \Delta:(\mathbf{x}, \mathbf{x}) \in \Theta^{N E}\right\} .
$$

Nicht alle Gleichgewichte eines symmetrischen Spiels müssen symmetrisch sein, doch aus dem Fixpunktsatz von Kakutani ergibt sich, dass es mindestens ein symmetrisches Nash-Gleichgewicht gibt.

\subsubsection{Kritik der klassischen Spieltheorie und evolutionäre Spieltheorie}

So bestechend das Nash-Gleichgewicht auch erscheinen mag, das Konzept birgt ein großes Problem. Nur in Ausnahmefällen gibt es ein eindeutiges Nash-Gleichgewicht - Koordinationsspiele gehören nicht zu diesen Ausnahmen, wie später diskutiert wird. Diese Situation ist unbefriedigend, denn Definition 5.2 gibt keine Hinweise darauf, welches Gleichgewicht zum tatsächlichen Spielausgang werden könnte. Nicht minder schwerwiegend ist die Tatsache, dass in mehrrundigen Spielen oder bei unvollständiger Information nicht alle Nash-Gleichgewichte plausibel sind. Auch dies wird später anhand eines Beispiels belegt.

Um unplausible Nash-Gleichgewichte auszusortieren, wurde das Konzept verfeinert. Die bekanntesten dieser sogenannten Refinements sind die Teilspielperfektheit ${ }^{296}$, die Trembling-Hand-Perfektheit ${ }^{297}$, das propere Gleichgewicht $^{298}$ und das sequentielle Gleichgewicht ${ }^{299}$. Sie haben nicht das Ziel, eine eindeutige Lösung zu ermitteln; gelegentlich eliminiert ein Refinement sogar alle Lösungen eines Spiels.

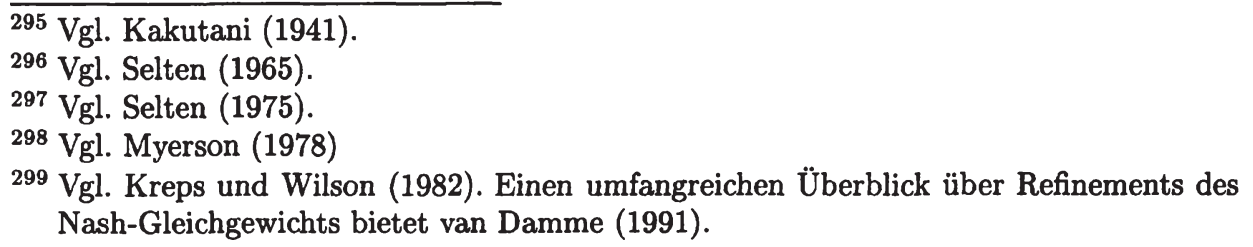


Um zu eindeutigen Lösungen zu kommen, sind verschiedene Methoden entwickelt worden. Man kann Gleichgewichte auf Prominenz untersuchen, die sich auf außerhalb des Spiels liegende, oftmals psychologische Fakten stützt. Solche Gleichgewichte werden als Fokuspunkte bezeichnet. ${ }^{300}$ Die Idee lässt sich jedoch kaum formalisieren, weshalb sie in der Spieltheorie nicht weiter verfolgt worden ist.

Ein weiterer Weg besteht darin, sinnvolle Anforderungen in Form von Axiomen an die Lösung zu stellen. ${ }^{301}$ Der Vorteil dieser Methode liegt darin, dass die Auswahlkriterien durch die strenge Axiomatisierung offengelegt werden und damit kritisierbar sind. Dies ist bei der Auswahl eines Fokuspunktes aufgrund außerhalb des Spiels liegender Einflüsse weniger möglich. Anhand eines Koordinationsspiels wird weiter unten die Problematik dieser Methode verdeutlicht, die darin liegt, dass die zugrunde gelegte Axiomatik in sich widersprüchlich sein kann.

Ein Ansatz, der sich in jüngerer Zeit als recht fruchtbar erwiesen hat, ist die evolutionäre Spieltheorie. ${ }^{302}$ Die evolutionsstabile Strategie ist ein Schlüsselkonzept der evolutionären Spieltheorie. Das Szenario sieht vor, dass aus einer großen Population zwei Spieler zufällig ausgewählt werden, die ein symmetrisches Zwei-Personen-Spiel spielen und dabei auf dieselbe Strategie $\mathbf{x} \in \Delta$ programmiert sind. Nun gebe es einen kleinen Anteil der Population, der eine andere Strategie $\mathbf{y} \in \Delta$ spielt. Die ursprüngliche Strategie $\mathbf{x}$ ist evolutionsstabil, wenn es eine Grenze $\epsilon>0$ gibt, unterhalb derer die Auszahlung eines solchen Anteils, der $\mathbf{y}$ spielt, geringer ist als die des Anteils, der $\mathbf{x}$ spielt. Man stellt sich dabei eine Verbindung zwischen Auszahlung und Verbreitung einer Strategie vor, sei es in biologischem Kontext durch Fortpflanzung, sei es in ökonomischem Kontext durch Nachahmung. ${ }^{303}$

$\overline{300}$ Der Ansatz geht zurück auf Schelling (1960). Ein instruktives Beispiel nach Schelling findet sich in Rasmusen (1994), S. 28, in dem Spieler eine Zahl aus der Reihe 7, 100, 13, 261, 99, 666 wählen müssen und gewinnen, wenn beide dieselbe Zahl wählen. Schelling identifizierte 7 als Fokuspunkt, in einer Gruppe von Satanisten könnte jedoch 666 der Fokuspunkt sein.

${ }^{301}$ Die axiomatische Gleichgewichtsauswahltheorie wurde in geschlossener Form erstmals von Harsanyi und Selten (1988) dargestellt und von Güth und Kalkofen (1989) weiterentwickelt. Für einen knappen Überblick vgl. Güth (1999), S. 179ff.

$302 \mathrm{Vgl}$. die umfassende Darstellung in Weibull (1995) oder die kurzen Einführungen Vilks und Clausing (1999) und Steven und Otterpohl (2000b).

${ }^{303}$ Vgl. zum Folgenden Weibull (1995), S. 36ff. 


\section{Definition 5.3}

$\mathbf{x} \in \Delta$ ist evolutionär stabil, wenn es für jede Strategie $\mathbf{y} \neq \mathbf{x}$ ein $\bar{\epsilon}_{\mathbf{y}}$ gibt, so dass für alle $\epsilon \in\left(0, \bar{\epsilon}_{\mathbf{y}}\right)$ gilt:

$$
u(\mathbf{x}, \epsilon \mathbf{y}+(1-\epsilon) \mathbf{x})>u(\mathbf{y}, \epsilon \mathbf{y}+(1-\epsilon) \mathbf{x})
$$

Die evolutionsstabilen Strategien werden in der Menge $\Delta^{E S S}$ zusammengefasst.

\section{Satz 5.2}

Für alle $\mathbf{x} \in \Delta^{E S S}$ und $\mathbf{y} \in \Theta$ gilt:

$$
\begin{gathered}
\forall \mathbf{y}: \quad u(\mathbf{x}, \mathbf{x}) \geq u(\mathbf{y}, \mathbf{x}) \\
\forall \mathbf{y} \neq \mathbf{x}: \quad u(\mathbf{x}, \mathbf{x})=u(\mathbf{y}, \mathbf{x}) \Rightarrow u(\mathbf{x}, \mathbf{y})>u(\mathbf{y}, \mathbf{y}) .
\end{gathered}
$$

Beweis. Wir zeigen, dass $\mathbf{x} \notin \Delta^{E S S}$, wenn eine der beiden Bedingungen nicht erfüllt ist. Sei zunächst $u(\mathbf{x}-\mathbf{y}, \mathbf{x})<0$. Es gilt

$$
\lim _{\epsilon \rightarrow 0} \epsilon \cdot u(\mathbf{x}-\mathbf{y}, \mathbf{y}-\mathbf{x})=0
$$

also gibt es ein $\bar{\epsilon}$, so dass für alle $\epsilon \in(0, \bar{\epsilon})$ gilt:

$$
\epsilon \cdot u(\mathbf{x}-\mathbf{y}, \mathbf{y}-\mathbf{x})<-u(\mathbf{x}-\mathbf{y}, \mathbf{x}) \Longleftrightarrow u(\mathbf{x}-\mathbf{y}, \epsilon \mathbf{y}+(\mathbf{1}-\epsilon) \mathbf{x})<0 .
$$

Dies ist ein Widerspruch zu Ungleichung (5.1).

Sei nun $\mathbf{y} \neq \mathbf{x}, u(\mathbf{x}-\mathbf{y}, \mathbf{x})=0$ und $u(\mathbf{x}-\mathbf{y}, \mathbf{y})<0$. Dann ist

$$
(1-\epsilon) u(\mathbf{x}-\mathbf{y}, \mathbf{x})+\epsilon \cdot u(\mathbf{x}-\mathbf{y}, \mathbf{y})<0 \Longleftrightarrow u(\mathbf{x}-\mathbf{y}, \epsilon \mathbf{y}+(\mathbf{1}-\epsilon) \mathbf{x})<0
$$

für alle $\epsilon \in(0,1)$ im Widerspruch zu (5.1).

Evolutionäre Stabilität ist eine statische Beschreibung des dynamisch stabilen Resultats der Selektion. ${ }^{304}$ Satz 5.2 hat gezeigt, dass es sich um ein Refinement des Nash-Gleichgewichts handelt. Die Bedeutung liegt darin, dass es sich in natürlicher Weise mit einer Dynamik zur Beschreibung des Anpassungsprozesses an das Gleichgewicht verbinden lässt.

\section{Corollar 5.3}

Wenn $(\mathbf{x}, \mathbf{x}) \in \Theta^{N E}$ ein striktes Nash-Gleichgewicht ist, dann gilt $\mathbf{x} \in \Delta^{E S S}$. 
Beweis. Die erste Bedingung aus Satz 5.2 besagt, dass jede evolutionsstabile Strategie nash-gleichgewichtig ist. Auf ein striktes Nash-Gleichgewicht gibt es keine alternative beste Antwort, so dass die zweite Bedingung ebenfalls erfüllt ist.

Beide Gleichgewichtskonzepte genügen gewissen Invarianzeigenschaften. Eine positiv affine Transformation der Auszahlungen lässt die Lösungen ebenso unverändert wie die Addition einer Konstanten zu allen Auszahlungen eines Spielers $i$, die zu einem fixen Strategienprofil $\mathbf{s}_{-i}$ der anderen Spieler gehören. ${ }^{305}$ Daraus ergibt sich die Möglichkeit, Äquivalenzklassen von Spielen gemäß den Gleichgewichten zu bilden und diese Äquivalenzklassen anhand von Repräsentanten mit normierten Auszahlungsfunktionen zu analysieren.

\subsubsection{Evolutorische Ökonomik und evolutionäre Spieltheorie}

Das ursprüngliche Anwendungsgebiet der evolutionären Spieltheorie ist die Analyse von Konflikt und Kooperation in Flora und Fauna. ${ }^{306}$ Bei der Betrachtung von Tieren und Pflanzen macht die in der Spieltheorie übliche Annahme der Rationalität offensichtlich wenig Sinn. Stattdessen werden Strategien als ererbte Verhaltensmuster betrachtet; die Spieler sind nicht in der Lage, von ihrer Programmierung abzuweichen. Die Auszahlungen des Spiels werden als Fitness interpretiert, nach der sich die Reproduktionswahrscheinlichkeit bemisst. Unterlegene Spieler sterben mitsamt ihren Strategien in einer Darwinschen Selektion aus. Neue Strategien entstehen durch zufällige Mutationen, die sich durch Reproduktion ausbreiten, sofern sie sich als den alten Strategien überlegen erweisen.

Die evolutionäre Spieltheorie hat in den letzten Jahren zunehmend Einzug in die Ökonomik gehalten. Evolutorische Ansätze sind in den Wirtschaftswissenschaften alles andere als neu ${ }^{307}$ Im Gegenteil wurde die Lehre Darwins ${ }^{308}$ bereits von Malthus und Smith beeinflusst ${ }^{309}$. Im 20. Jahrhundert wurden evolutorische Theorien weitgehend von der statischen Gleichgewichtsanalyse überdeckt, die ihre Vorbilder in der Physik findet ${ }^{310}$ und deren Ideen

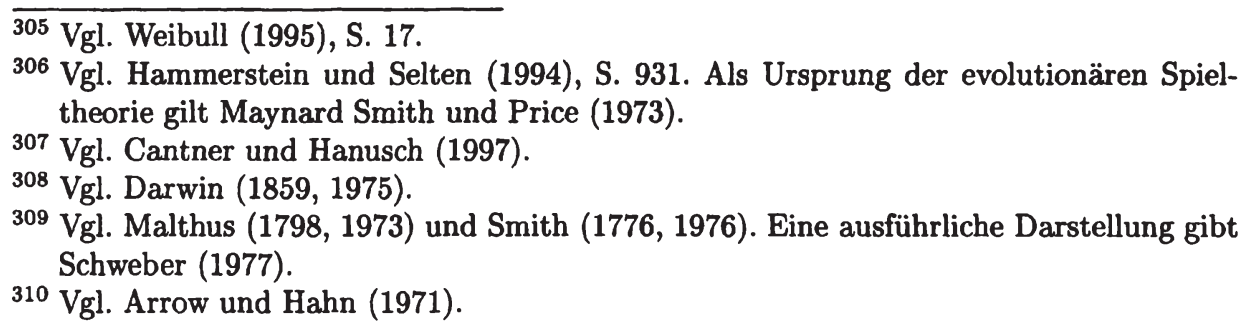


auch in die Betriebswirtschaftslehre transferiert wurden ${ }^{311}$. Die Erfolge dieser Theorierichtung sind unbestreitbar; folglich wurden grundlegende Annahmen von der Methode der klassischen Spieltheorie aufgegriffen, insbesondere die Common-Knowledge-Annahme der Rationalität.

Es gibt jedoch schwerwiegende Einwände gegen das Konzept der vollständigen Rationalität. Aus theoretischer Sicht ist die inhärente Widersprüchlichkeit zu bemängeln. ${ }^{312}$ Ein vollständig rationales Individuum wird nicht von seiner Gleichgewichtsstrategie abweichen. Die Definition des Gleichgewichts kommt aber nicht aus, ohne irrationales Verhalten in Betracht zu ziehen. ${ }^{313}$ Dieser Einwand mag noch esoterisch anmuten ${ }^{314}$, dennoch wird zweifelhaft, inwieweit vollständige Rationalität überhaupt einen sinnvollen Begriff darstellt.

Das Nash-Gleichgewicht gibt außerdem wie alle seine Refinements keine Auskunft darüber, auf welchem Wege es erreicht wird. Um es direkt auf die individuellen Entscheidungen zurückzuführen, müssen starke Informationsanforderungen erfüllt werden, was als ernsthafte Schwäche des Lösungskonzeptes angesehen wird. ${ }^{315}$ Dieser Einwand trifft ebenso die vornehmlich statisch ausgerichtete Mikroökonomik.

Aus empirischer Sicht erheben sich ebenfalls Zweifel an der Rationalitätsannahme. Sicherlich bedarf die Annahme, Menschen verhielten sich nicht rational, der Begründung. Doch zahlreiche Experimente stützen die These, dass Menschen optimale Strategien nicht durch sorgfältiges Vorausbedenken ihrer Handlungen, sondern durch einen Prozess von Versuch und Irrtum finden. ${ }^{316}$

Aufgrund der genannten Schwächen rationalitätsorientierter Konzepte sind evolutorische Theorien, die die Biologie zum Vorbild wählen, weiter verfolgt worden ${ }^{317}$ und erleben in den letzten Jahren eine Renaissance ${ }^{318}$. Die Ansätze sind vielfach kritisiert worden, da die behaupteten Analogien zwischen biologischer Evolution und ökonomischer Entwicklung nicht gegeben

311 Vgl. Gutenberg (1989).

312 Vgl. Binmore (1987), S. 181.

${ }^{313}$ Dies wird unmittelbar einsichtig, wenn man Trembling-Hand-perfekte Gleichgewichte betrachtet.

${ }^{314}$ Selten (1975), S. 35, betrachtet vollständige Rationalität als Grenzfall unvollständiger Rationalität, in dem gleichsam die Wahrscheinlichkeit irrationalen Verhaltens gegen null strebt.

315 Vgl. Binmore $(1987,1988)$.

${ }^{316}$ Vgl. das Vorwort von Binmore in Weibull (1995), S. ix.

${ }^{317}$ Vgl. z.B. Alchian (1950), Penrose (1952), Nelson und Winter (1982).

318 Vgl. Friedman (1998b). 
seien. ${ }^{319}$ Evolution vollzieht sich durch die Weitergabe von Genen durch Reproduktion. Dabei werden schlechte Erbanlagen durch eine geringere Reproduktionswahrscheinlichkeit im Laufe der Zeit verschwinden, gute Erbanlagen sich durchsetzen. Neue Erbanlagen entstehen durch zufällige Mutationen, deren Güte im Selektionsprozess erprobt wird. Nun darf man die Übertragung genetischer auf ökonomische Sachverhalte nicht so weit treiben, dass man zu der Redeweise greift, Unternehmen strebten danach, ihre Gene an Nachkommen zu vererben. ${ }^{320}$ Auch für die Erklärung menschlichen Verhaltens ist eine allzu wörtliche Übertragung wegen der sehr langen Frist evolutionärer Anpassungsprozesse wertlos.

Statt dessen kann man den drei biologischen Begriffen Reproduktion, Selektion und Mutation die Begriffe Imitation, Elimination und Innovation gegenüberstellen. Nicht Unternehmen reproduzieren sich, sondern Produkte, Produktionsmethoden, Organisationsformen usw. - kurz: Strategien - werden nachgeahmt. Innovationen werden als zielgerichtete Experimente aufgefasst, die sich als erfolgreich gegenüber dem Hergebrachten erweisen können oder wieder verschwinden. Für die Elimination unterlegener Strategien sorgt der Wettbewerb, der Unterlegenheit mit Verlusten und schließlich Konkurs bestraft. Auf diese Weise lässt sich selbstverständlich nicht jedes (wirtschaftliche) Handeln erklären. In der vereinfachenden Analogie bleiben Fragen ausgeklammert, wie Innovationen zustande kommen oder welche Rolle zielgerichteter Planung zukommt. Hinsichtlich solcher Elemente sind biologische Ideen abzuwandeln, um ökonomische Prozesse erklären zu können. ${ }^{321}$ Die zugrunde liegenden formalen Strukturen sind jedoch hinreichend ähnlich, um die Verwendung derselben Methode, der evolutionären Spieltheorie, geboten erscheinen zu lassen.

Hier soll nicht die Diskussion nachgezeichnet werden, inwieweit sich der evolutorische Ansatz von der herrschenden Lehre vereinnahmen lassen sollte. $^{322}$ Doch schon die Tatsache, dass evolutionsstabile Strategien nashgleichgewichtig sind, zeigt, dass beide Ansätze nicht so weit voneinander entfernt sein können. Vielleicht liegt die Ursache des Erfolges der rationalitätsorientierten Theorien sogar darin, dass evolutionäre Prozesse nur solches Verhalten langfristig in Erscheinung treten lassen.

\footnotetext{
319 Vgl. Schneider (1997b), S. 27ff.

320 Vgl. kritisch Penrose (1952).

321 Vgl. zu einer Kritik Brenner (1998).

322 Vgl. hierzu Friedman (1998b).
} 


\subsubsection{Koordinationsspiele}

Die Spieltheorie bildet das Problem der Koordination ab, indem die Regeln verlangen, dass die Spieler sich auf bestimmte Strategienkombinationen einigen müssen, um ihre Auszahlungen zu maximieren. Zur Einführung können wir uns auf Zwei-Personen-Spiele in Bimatrix-Form beschränken. Die Spieler werden mit Großbuchstaben, die Strategien mit Kleinbuchstaben bezeichnet, um sie von den Auszahlungen abzuheben. Zunächst werden die wichtigsten Beispiele mit passenden Cover Stories vorgestellt. Dann wird gezeigt, dass man alle diese Varianten durch geeignete Normierung derselben BimatrixForm zuordnen kann, die abschließend auf die vorgestellten Lösungskonzepte hin untersucht wird.

\subsubsection{Beispiele}

Zunächst die wichtigsten Beispiele: Das einfachste Koordinationsspiel wird in Abbildung 5.1 beschrieben, wobei der besseren Lesbarkeit wegen die Auszahlungen an beide Spieler aufgeführt sind:

\begin{tabular}{|cccc|}
\hline & \multicolumn{3}{c}{ Spieler B } \\
& & a & b \\
Spieler A & a & $\mathbf{1 , 1}$ & 0,0 \\
& b & 0,0 & $\mathbf{1 , 1}$ \\
& & & \\
\hline
\end{tabular}

Abbildung 5.1: Pure Coordination

Es beschreibt z.B. die Situation zweier Personen, die sich an einem von zwei möglichen Orten treffen wollen, sich aber nicht absprechen können. Ein anderes Beispiel wären zwei Autoren, die einen gemeinsamen Text verfassen wollen und die Wahl zwischen zwei Textverarbeitungssystemen haben.

Pure Coordination lässt sich auf vielfältige Weise variieren. Eine erste Abwandlung bestünde darin, die Symmetrie zwischen den Lösungen aufzuheben, wie in Abbildung 5.2 dargestellt.

Hier zeichnet sich das Strategienprofil $(b, b)$ durch höhere Auszahlungen an beide Spieler aus. Nichts aber garantiert, dass dieses Profil auch wirklich gespielt wird. Im Gegenteil, hat ein Spieler den Anzugsvorteil und wählt 


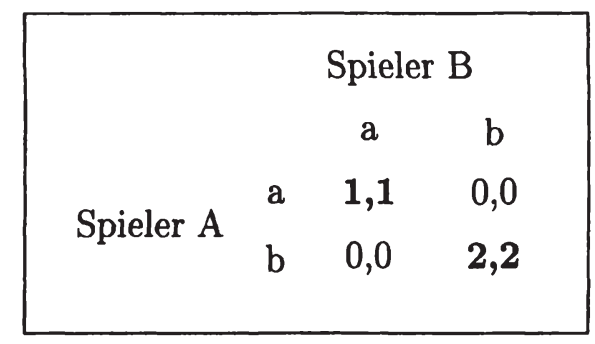

Abbildung 5.2: Win-Win-Game

aus irgendeinem Grund Strategie a, so muss der andere Spieler folgen. Man bezeichnet eine Situation, in der die Spieler in einem ineffizienten Strategienprofil gefangen sind, als Lock-in. ${ }^{323}$ Ein isoliertes Abweichen eines Spielers aus dem Lock-in würde ihm eine geringere Auszahlung bescheren.

Eine Variante, in der der Anzugsvorteil von entscheidender Bedeutung ist, zeigt Abbildung 5.3. Die Strategienprofile $(a, a)$ und $(b, b)$ sind auf eine ganz spezielle Weise asymmetrisch: Jedes gibt einem der Spieler einen Vorteil; dennoch ist es für den anderen Spieler immer noch die beste Antwort, dem ersten Spieler zu folgen.

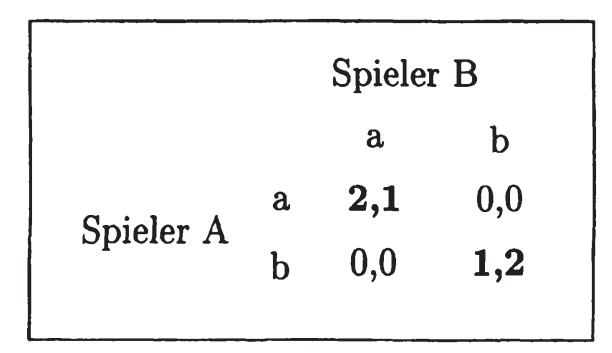

Abbildung 5.3: Battle of Sexes

Der Name des Spiels stammt von der üblichen Cover Story, in der die beiden Spieler ein (Ehe-)Paar sind und die beiden Strategien ,ins Theater gehen" bzw. ,einen Boxkampf anschauen“. Obwohl aber der Mann zum Boxen und die Frau ins Theater möchte, hängen sie doch so sehr aneinander, dass sie lieber mit dem anderen mitgehen, als allein ihren Willen durchsetzen. Wer hier nun einen Anzugsvorteil besitzt (z.B. weil er oder sie am schnellsten die Eintrittskarten kauft), setzt sich durch.

${ }^{323}$ Vgl. Rieck (1993), S. 50. 


\subsubsection{Normierung und Lösungen}

Koordinationsspiele mit zweielementigen Strategienmengen $S_{1}$ und $S_{2}$ sind bei normierten Auszahlungen doppelsymmetrisch mit der Matrix

$$
\mathbf{A}=\left(\begin{array}{cc}
a_{1} & 0 \\
0 & a_{2}
\end{array}\right)
$$

Das normierte Koordinationsspiel hat drei symmetrische NashGleichgewichte: $\Delta^{N E}=\left\{\mathbf{e}^{1}, \mathbf{e}^{2}, \mathbf{x}\right\}$ mit $\mathbf{x}=\left(a_{2} /\left(a_{1}+a_{2}\right), a_{1} /\left(a_{1}+a_{2}\right)\right)$, wie man leicht nachprüft. ${ }^{324}$ Die beiden Gleichgewichte in reinen Strategien sind strikt, wohingegen jedes $\mathbf{y} \in \Delta$ beste Antwort auf das gemischte Gleichgewicht $\mathbf{x}$ ist. Das gemischte Gleichgewicht ist daher lediglich nicht selbstzerstörend.

Die strikten Gleichgewichte $\left(\mathbf{e}^{1}, \mathbf{e}^{1}\right)$ und $\left(\mathbf{e}^{2}, \mathbf{e}^{2}\right)$ sind nach Corollar 5.3 evolutionsstabil. Für das gemischte Gleichgewicht $\mathbf{x}$ gilt jedoch für die beste Antwort $\mathbf{e}^{1}$, dass $u\left(\mathbf{e}^{1}, \mathbf{e}^{1}\right)=a_{1}>a_{1} \cdot a_{2} /\left(a_{1}+a_{2}\right)=u\left(\mathbf{e}^{1}, \mathbf{x}\right)$, also $\mathbf{x} \notin \Delta^{E S S}$.

Schon das einfachste Koordinationsspiel (Abbildung 5.1) zeigt das grundlegende Problem auf: Es gibt mehrere Gleichgewichte. Erfolgreiche Koordination bedeutet in spieltheoretischem Kontext, eines dieser Gleichgewichte auszuwählen. Eine Veränderung der Auszahlungen gemäß Abbildung 5.2 ändert nichts an der Anzahl der Gleichgewichte, das Strategienprofil $\left(\mathbf{e}^{1}, \mathbf{e}^{1}\right)$ ist jedoch pareto-superior. Damit wird es zu einem Fokuspunkt; nichtsdestoweniger lohnt sich ein einseitiges Abweichen von Profil $\left(\mathbf{e}^{2}, \mathbf{e}^{2}\right)$ nicht. Koordination lässt sich in beiden Varianten leicht durch Kommunikation herstellen. In der Spieltheorie wird die Kommunikation vor der Durchführung der Handlungen mit dem Ziel der wechselseitigen Abstimmung als cheap talk bezeichnet ${ }^{325}$, was eine ganz wesentliche Voraussetzung betont, nämlich dass die Kosten der Kommunikation vernachlässigbar sein müssen in dem Sinne, dass die Veränderung der Auszahlungen die Gleichgewichte nicht verändern darf.

Sich allein auf Kommunikation zu verlassen, birgt jedoch Probleme. Hebt man die Symmetrie der Gleichgewichte auf wie in Abbildung 5.3, bevorteilt jedes Nash-Gleichgewicht einen der Spieler. Es ist zweifelhaft, ob durch einfache Kommunikation eine Einigung erzielt werden kann. Hier wird sich der Einflussreichere durchsetzen, den es auch ohne formale Hierarchie geben dürfte. Die Spieltheorie modelliert dies dadurch, dass die Züge nicht mehr gleichzeitig durchgeführt werden. Streng genommen wird diese Frage von

324 Zur Berechnung gemischter Gleichgewichte vgl. z.B. Rieck (1993), S. 56.

325 Vgl. Rasmusen (1994), S. 76. 
Abbildung 5.3 nicht eingefangen, da die dort unterstellten Strategienmengen gleichzeitige Züge implizieren. Die Analyse verkompliziert sich durch sequentielle Züge jedoch. Der Anziehende hat nach wie vor die Wahl zwischen den Strategien $a$ und $b$, während der Nachziehende die Strategien $(a, a),(a, b)$, $(b, a)$ und $(b, b)$ zur Wahl hat. Dabei bedeutet $(b, a)$, dass der Nachziehende $b$ wählt, wenn der Anziehende a spielt, und $a$, wenn $b$ gespielt wird. Abbildung 5.4 zeigt die Bimatrix-Form.

\begin{tabular}{|cccccc|}
\hline & & \multicolumn{5}{c}{ Spieler B } \\
& & $(\mathrm{a}, \mathrm{a})$ & $(\mathrm{a}, \mathrm{b})$ & $(\mathrm{b}, \mathrm{a})$ & $(\mathrm{b}, \mathrm{b})$ \\
& $\mathrm{a}$ & $\mathbf{2 , 1}$ & $\mathbf{2 , 1}$ & 0,0 & 0,0 \\
Spieler A & $\mathrm{b}$ & 0,0 & 1,2 & 0,0 & $\mathbf{1 , 2}$ \\
& & & & & \\
\hline
\end{tabular}

Abbildung 5.4: Follow the Leader

Dieses Spiel hat drei Nash-Gleichgewichte in reinen Strategien: $\{a,(a, a)\}$, $\{b,(b, b)\}$ und $\{a,(a, b)\}$. Die beiden ersten erscheinen jedoch nicht sinnvoll, da der Nachziehende die Aktion des Anziehenden nicht berücksichtigt. ${ }^{326}$ Legt man die Verfeinerung der Teilspielperfektheit zugrunde ${ }^{327}$, erhält man nur noch das sinnvolle Gleichgewicht $\{a,(a, b)\}$ mit den Payoffs 2 für Spieler A und 1 für Spieler B. Damit erreicht man, dass der Nachziehende sich auch außerhalb des Gleichgewichtspfades rational verhält, und die Spielergebnisse sind auch dann sinnvoll, wenn der Anziehende möglicherweise einen Fehler bei der Wahl seiner Aktion macht. ${ }^{328}$

Wenn man das Win-Win-Game variiert wie in Abbildung 5.5, zeigt sich, dass Pareto-Effizienz ein fragwürdiges Kriterium sein kann. Auch hier gibt es ein pareto-superiores Strategienprofil (b,b), doch die Wahl der Strategie b ist riskant. Macht der andere Spieler einen Fehler und wählt Strategie a, erhält sein Gegner eine stark negative Auszahlung. Das Profil (a,a) heißt deshalb risikodominant. ${ }^{329}$

Das Gleichgewicht $(b, b)$ ist auszahlungsdominant und damit paretoeffizient. Andererseits geht ein Spieler, der Strategie b wählt, das Risiko ein,

326 Will man die Situation abbilden, dass der Nachziehende nicht in der Lage ist zu reagieren, etwa weil er die Aktion des Anziehenden nicht beobachten kann, muss man die Züge simultan ausführen lassen.

327 Das Konzept stammt von Selten (1965).

${ }^{328}$ Der Gedanke wird formalisiert in der „Trembling-Hand-Perfektheit“. Vgl. Selten (1975). $329 \mathrm{Vgl}$. Holler und Iling (1993), S. 138. 


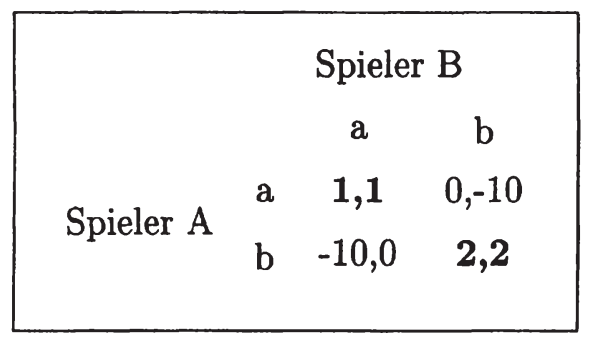

Abbildung 5.5: Dangerous Coordination

eine Auszahlung von -10 zu erhalten, wenn der Gegner abweicht. Bei Gleichgewicht $(\mathrm{a}, \mathrm{a})$ ist ein solcher Verlust kleiner, so dass dieses risikodominant ist. Die beiden Axiome führen also zu widersprüchlichen Ergebnissen. Harsanyi und Selten lösen das Problem, indem sie eine strikte Hierarchie der Axiome verwenden, in der der Auszahlungsdominanz Priorität eingeräumt wird. ${ }^{330}$ Dies ist jedoch nicht unbestritten geblieben ${ }^{331}$, zumal Experimente bestätigen, dass Spieler eher das risikodominante Gleichgewicht wählen ${ }^{332}$. Außerdem ergibt die Normalisierung des Spiels $a_{1}=11$ und $a_{2}=2$, und man kann damit argumentieren, dass das risikodominante Gleichgewicht das eigentlich rationale ist. ${ }^{333}$

\subsubsection{Ein konvergentes Koordinationsmodell}

Beide statischen Gleichgewichtskonzepte sind nicht in der Lage vorauszusagen, welches Gleichgewicht nun tatsächlich gespielt wird. Sie können weder erklären, wie ein Lock-in im Win-Win-Game zustandekommt, noch wie der Konflikt im Battle of Sexes gelöst wird. Der Vorteil der Evolutionsstabilität liegt aber darin, dass sie sich in natürlicher Weise mit einer Dynamik verbinden lässt. Die Hypothese der Replikator-Dynamik lautet, dass die Wachstumsrate der Wahrscheinlichkeit $x_{i}$, mit der eine reine Strategie $i$ gewählt wird, sich proportional zur erwarteten Auszahlungsdifferenz zwischen reiner Strategie $i$ und vorherrschender gemischter Strategie $\mathbf{x}$ verhält ${ }^{334}$ :

$$
\frac{\dot{x}_{i}}{x_{i}}=u\left(\mathbf{e}^{i}, \mathbf{x}\right)-u(\mathbf{x}, \mathbf{x})
$$

330 Vgl. Harsanyi und Selten (1988).

331 Vgl. Holler und Illing (1993).

332 van Huyck, Battalio und Beil (1990).

333 Vgl. Weibull (1995), S. 31.

${ }^{334}$ Diese Differenz wird in biologischem Kontext als relative Fitness interpretiert. Vgl. Friedman (1998a), S. 24. 
Die Replikator-Dynamik besitzt gewisse Invarianzeigenschaften, die eine Normalisierung der Auszahlungen erlauben. ${ }^{335}$ Für das normalisierte Koordinationsspiel lautet die Replikator-Dynamik

$$
\begin{gathered}
\dot{x}_{1}=\left(a_{1} x_{1}-a_{2} x_{2}\right) x_{1} x_{2}, \\
\dot{x}_{2}=-\dot{x}_{1} .
\end{gathered}
$$

Man erkennt, dass alle drei Nash-Gleichgewichte Steady States darstellen, das gemischte Gleichgewicht jedoch instabil unter der Replikator-Dynamik ist. Dies stimmt überein mit den Beobachtungen zur Evolutionsstabilität.

Außerdem sieht man, dass

$$
\begin{aligned}
& \lim _{t \rightarrow \infty} \mathbf{x}=\mathbf{e}^{1} \text { für } x_{1}^{0}>\frac{a_{2}}{a_{1}+a_{2}}, \\
& \lim _{t \rightarrow \infty} \mathbf{x}=\mathbf{e}^{2} \text { für } x_{1}^{0}<\frac{a_{2}}{a_{1}+a_{2}} .
\end{aligned}
$$

Die Replikator-Dynamik bestimmt, welches strikte Nash-Gleichgewicht den eindeutigen Grenzwert bildet. Das Konzept der Evolutionsstabilität in Verbindung mit der Replikator-Dynamik garantiert ein eindeutiges Ergebnis. Man kann nun die Existenz eines Lock-in durch die Anfangsbedingung erklären. Wenn die Wahrscheinlichkeit, dass die pareto-inferiore Strategie gespielt wird, hinreichend groß ist, ist die erwartete Auszahlung der pareto-superioren Strategie entsprechend klein, und die inferiore Strategie wird noch häufiger gewählt, bis das Lock-in erreicht ist. Die Anfangsbedingung selbst ist modellexogen, doch sie präzisiert die Umstände, unter denen es zum Lock-in kommt. Analog ergeben sich die Bedingungen, unter denen sich ein Spieler im Battle of Sexes durchsetzt.

\subsection{Koordination mit chaotischer Dynamik}

Die nachfolgenden Modelle wollen zeigen, dass chaotisches Verhalten prinzipiell bei keinem Koordinationsinstrument ausgeschlossen werden kann. Mit Schäffer unterscheiden wir vertikale Koordination sowie horizontale Koordination ohne und mit gemeinsamem Zweck. ${ }^{336}$ Die Modelle $\mathcal{B}$ (Abschnitt

335 Vgl. Weibull (1995), S. 73.

${ }^{336}$ Es sei daran erinnert, dass nach Schäffer (1996) vertikale Koordination einen gemeinsamen Zweck voraussetzt. Fehlt dieser, erfolgt die Koordination durch Zwang, was in einer freiheitlichen Gesellschaft unzulässig ist. Vgl. auch nochmals Abbildung 4.1 auf S. 111. 
5.2.1) zur Budgetierung, $\mathcal{V}($ Abschnitt 5.2.2) zur Koordination über Verrechnungspreise und $\mathcal{S}$ (Abschnitt 5.2.3) zur Selbstabstimmung korrespondieren mit den drei besetzten Feldern der Matrix nach Schäffer.

\subsubsection{Koordination durch Budgetierung}

\subsubsection{Modellformulierung}

Der Begriff der Budgetierung wird in der Literatur nicht einheitlich verwendet. Bisweilen wird Budgetierung mit der Gesamtplanung der Unternehmung gleichgesetzt, bisweilen beschränkt man sich auf die kurzfristige Planung. Gängiger als diese Auffassungen ist das Verständnis als Koordinationsinstrument, bei dem die an die nachgeordneten Instanzen delegierten Teilplanungen koordiniert werden, indem von der Zentrale Vorgaben hinsichtlich bestimmter Plangrößen gemacht werden. ${ }^{337}$

Mit Budgetierung wird hier die Zuweisung finanzieller Ressourcen an eine Abteilung wie Marketing oder Forschung oder eine Sparte respektive ein Profit Center - im Folgenden pars pro toto Abteilung genannt - bezeichnet. Damit wird faktisch eine Verschuldungsgrenze der Abteilungen gegenüber der Zentrale bzw. dem Unternehmen festgelegt. Die Allokation der vorhandenen Ressourcen erfolgt durch eine Budgetplanung auf übergeordneter Ebene, wobei hier die Frage der Partizipation der betroffenen Abteilungen und damit die Problematik asymmetrischer Informationen ausgeklammert bleibt. Die zentrale Planung gewährleistet eine konsistente Budgetierung und somit eine Koordination der Abteilungen. ${ }^{338}$

Im Folgenden wird ein Partialmodell $\mathcal{B}$ betrachtet, das die Budgetzuteilung einer Abteilung in dynamischem Kontext abbildet. Es wird unterstellt, dass die Budgethöhe von einer Zielgröße abhängt, die wiederum von der Budgethöhe beeinflusst wird. Solche Regeln sind in der Praxis häufig anzutreffen, z.B. wird das Marketingbudget vom Umsatz der Vorperiode bestimmt, das Forschungsbudget vom Gewinn der Vorperiode oder das Verwaltungsbudget von den Ausgaben der Vorperiode.

Zunächst stellen wir uns als Beispiel das Werbebudget $W$ und als Zielerreichungsgrad die Höhe des Gewinns $G$ vor. Intensivere Werbung erhöht den Absatz und damit den Erlös $E$, wobei man abnehmende Erlöszuwächse

337 Vgl. Serfling (1992), S. 251.

338 Vgl. Ewert und Wagenhofer (2000), S. 458. 
unterstellen kann:

$$
E=E(W), \quad E^{\prime}(W)>0, \quad E^{\prime \prime}(W)<0 .
$$

Damit wird angenommen, dass jede Intensivierung der Werbeaktivitäten zusätzliche Kunden akquiriert, die Anzahl der Neukunden bzw. die von den Kunden zusätzlich nachgefragte Menge aber immer geringer wird. Hier wird eine Analogie zum Ertragsgesetz der neoklassischen Produktionstheorie gebildet. ${ }^{339}$ Man kann die Annahme abnehmender Grenzerlöse für kleine Werbebudgets aber auch bezweifeln. Sehr geringe Werbeaktivitäten bleiben für die meisten potenziellen Kunden unterhalb der Wahrnehmungsschwelle. Von einem solch geringen Niveau aus hat eine Erhöhung des Budgets nur eine kleine Wirkung auf den Erlös. Wenn die Wahrnehmungsschwelle aber überschritten wird, kann man von größeren Grenzerlösen der Werbung ausgehen. Der Bekanntheitsgrad des Produktes steigt; die Werbung kann indirekte Wirkung entfalten, indem Nachfrager auftreten, die nicht von der Werbung selbst zum Kauf animiert wurden, sondern vom Kauf anderer Individuen. Für Budgets unterhalb einer gewissen Höhe sind also steigende Grenzerlöse plausibel, erst jenseits dieser Höhe tritt ein Sättigungseffekt ein, der die Grenzerlöse sinken lässt. Insgesamt ergibt sich so ein s-förmiger Verlauf der Erlösfunktion in Abhängigkeit vom Werbeaufwand, der dem klassischen Ertragsgesetz der Produktionstheorie ${ }^{340}$ entspricht.

Auf der anderen Seite verursacht die Werbung Kosten in Höhe von $K$. Zum einen geschieht dies direkt; die Werbeausgaben, die im Budget veranschlagt sind, werden von der Betriebsbuchhaltung als Kosten verbucht. Zum anderen entstehen über die höhere Absatz- und damit Produktionsmenge indirekt auf die Werbung zurückzuführende Kosten. Üblicherweise unterstellt man eine konvexe Kostenfunktion, hier allerdings in Abhängigkeit von $W$ statt von der Ausbringungsmenge:

$$
K=K(W), \quad K^{\prime}(W)>0, \quad K^{\prime \prime}(W)>0 .
$$

Wenn man nun annimmt, dass auch bei s-förmigem Verlauf der Erlösfunktion die Grenzerlöse der Werbung für kleine Budgets immer größer sind als die Grenzkosten, dann ist die Gewinnfunktion als Differenz von Erlös und Kosten unimodal mit einem eindeutigen globalen Maximum. ${ }^{341}$ Wie wir

339 Vgl. Steven (1998), S. 43

340 Vgl. Steven (1998), S. 25.

${ }^{341}$ Ein Modell zur Abbildung von Werbebudgets untersucht Kopel (1994), S. 24ff, allerdings in einem erheblich anderen Szenario. 
schon in Kapitel 3 am Beispiel des quadratischen Iterators (3.8) gesehen haben, sind unimodale Funktionen in einem rückgekoppelten Regelkreis anfällig für Chaos.

Kehren wir zurück auf die höhere Abstraktionsebene. Der funktionale Zusammenhang zwischen Budgethöhe und Zielerreichungsgrad wird von zwei gegenläufigen Effekten bestimmt. Zum einen wird unterstellt, dass ein höheres Budget ceteris paribus einen höheren Zielerreichungsgrad zur Folge hat. Zum anderen belastet der Verbrauch finanzieller Ressourcen unmittelbar den Zielerreichungsgrad. Eine höhere Budgetzuweisung führt zunächst zu einer besseren Zielerreichung. Der Grenznutzen, ausgedrückt als Erhöhung des Zielerreichungsgrades, ist höher als die Grenzkosten, ausgedrückt als Erhöhung des Budgets. Ab einer gewissen Budgethöhe kann sich dies jedoch in sein Gegenteil verkehren, da zusätzliche Mittel nicht mehr zielgerecht eingesetzt werden können und in anderen Abteilungen fehlen. Der Grenznutzen einer Budgeterhöhung liegt in diesem Bereich unterhalb der Budgeterhöhung selbst, d.h. der Nettogrenznutzen ist negativ.

Der Abteilung wird eine gewisse Myopie unterstellt. Sie ist nicht in der Lage, den Zusammenhang zwischen Budgethöhe und Zielerreichungsgrad hinreichend zu überblicken, um Budgetmaximierung über mehrere Perioden hinweg betreiben zu können; das heißt, es ist ihr nicht möglich, durch die konkrete Verwendung der zugewiesenen finanziellen Mittel, mitunter auch durch Einsparungen und Verlagerung der Ausgaben in spätere Perioden, den Zielerreichungsgrad und damit die Budgetzuteilung langfristig hochzuhalten. Statt dessen gibt sie das zugeteilte Budget vollständig aus, um keine Budgetkürzung oder sogar Kompetenzbeschneidung hinnehmen zu müssen.

Von der Unternehmensleitung wird der Zielerreichungsgrad als Maß für die künftige Budgetzuweisung angesehen. Je erfolgreicher eine Abteilung gewirtschaftet hat, desto mehr finanzielle Mittel werden ihr in der Folgeperiode anvertraut.

Wir unterstellen mit den geschilderten Annahmen einen unimodalen $\mathrm{Zu}$ sammenhang zwischen Budgethöhe und Zielerreichungsgrad und eine monotone Funktion zwischen Zielerreichungsgrad und Budgetzuweisung für die nächste Periode. Dieses Szenario wird nun formalisiert.

Seien die Budgetmenge $B \subset \mathbb{R}_{0}^{+}$und die Menge der möglichen Zielerreichungsgrade $Z \subset \mathbb{R}$ kompakte Intervalle. Die Budgets sind also stets nichtnegativ; die Zielerreichungsgrade können jedes Vorzeichen annehmen und müssen nicht dieselbe Maßeinheit wie die Budgets besitzen. Letztere werden in Geldeinheiten gemessen, für die Zielerreichungsgrade sind neben finanziellen 
Größen Durchlauf- oder Lieferzeiten, Produktionsmengen oder bei weichen Zielen Punktzahlen aus Scoring-Verfahren denkbar.

Die Zeit $t$ wird in diskreten Schritten gemessen, also lassen sich die Bahnen der Budgets und Zielerreichungsgrade darstellen als die Folgen $\left\langle b_{t}\right\rangle_{t \in}, b_{t} \in B$ und $\left\langle z_{t}\right\rangle_{t \in}, z_{t} \in Z$.

Ferner unterstellen wir eine mindestens dreimal stetig differenzierbare ${ }^{342}$, unimodale Funktion $\phi: B \rightarrow Z, b_{t} \mapsto z_{t}$ und eine mindestens dreimal stetig differenzierbare, streng monoton steigende Funktion $\psi: Z \rightarrow B, z_{t} \mapsto b_{t+1} . \phi$ beschreibt den funktionalen Zusammenhang zwischen der Budgethöhe $b_{t}$ und dem damit maximal erreichbaren Zielerreichungsgrad $z_{t}$. Die Analogie zur Definition der Produktionsfunktion ${ }^{343}$ ist durchaus gewollt. In beiden Fällen steht ein Optimierungsgedanke im Hintergrund, hier die bestmögliche Nutzung der im Budget bereitgestellten Mittel, dort die effiziente Kombination der Produktionsfaktoren. Während jedoch für Produktionsfunktionen positive partielle Grenzproduktivitäten unterstellt werden, erreicht $\phi$ bei wachsen$\operatorname{dem} b$ ein Maximum und verläuft danach fallend. Analog zu diesem Verlauf wird im Modell von Day ${ }^{344}$ eine gesamtwirtschaftliche Produktionsfunktion mit einem fallendem Abschnitt verwendet. Dort wird argumentiert, dass ab einem gewissen Punkt eine weitere Erhöhung z.B. des Produktionsfaktors Kapital die natürlichen Ressourcen, also den Produktionsfaktor Boden, der in gesamtwirtschaftlichen Produktionsfunktionen normalerweise konstant gesetzt und nicht weiter betrachtet wird, so stark schädigt, dass der Nettoeffekt auf die Produktion negativ ist. Die Funktion $\psi$ verknüpft den Zielerreichungsgrad $z_{t}$ mit der Budgetzuweisung der Folgeperiode $b_{t+1}$. Sie beschreivi ids Verhalten der Unternehmensleitung und ist heuristischer, nicht optimierender Natur, da der Unternehmensleitung für ein Optimierungskalkül nicht genügend Informationen zur Verfügung stehen.

Aus der Verkettung von $\phi$ und $\psi$ ergibt sich als Modellbeschreibung die Differenzengleichung

$$
f=\psi \circ \phi: B \rightarrow B, b_{t} \mapsto b_{t+1},
$$

die die Budgethöhe in $t$ mit der Budgethöhe in $t+1$ verknüpft. Der nachfolgende Satz charakterisiert die allgemeinen Eigenschaften dieser Funktion. Die Untersuchung auf Chaos muss wie üblich anhand konkreter Funktionen

${ }^{342}$ Die Forderung nach dreifacher stetiger Differenzierbarkeit bedeutet keine wesentliche Einschränkung, sondern stellt sicher, dass alle verwendeten Ableitungen und Kompositionen wohldefiniert sind.

343 Vgl. z.B. Steven (1998), S. 6.

${ }^{344}$ Vgl. Day (1982) und oben Abschnitt 2.2.1. 
durchgeführt werden, die jedoch die Eigenschaften aus Satz 5.4 erfüllen müssen.

Satz 5.4

Die Funktion $f$ aus (5.2) ist mindestens dreimal stetig differenzierbar und unimodal.

Beweis. Aus $\phi \in C^{3}$ und $\psi \in C^{3}$ folgt ohne weiteres $\psi \circ \phi \in C^{3}$. Wegen der Differenzierbarkeit ist $\phi$ genau dann unimodal, wenn es einen Modus $b^{\text {mod }} \in B$ gibt mit

$$
\forall b_{t} \leqq b^{\bmod }: f^{\prime}\left(b_{t}\right) \gtreqless 0 .
$$

Wegen der strengen Monotonie von $\psi$ gilt $\psi^{\prime}\left(z_{t}\right)>0$ für alle $z_{t} \in Z$. Aus der Kettenregel erhält man

$$
f^{\prime}\left(b_{t}\right)=\psi^{\prime}\left(\phi\left(b_{t}\right)\right) \cdot \phi^{\prime}\left(b_{t}\right)
$$

und damit

$$
\forall b \in B: \quad \operatorname{sign} f^{\prime}\left(b_{t}\right)=\operatorname{sign} \phi^{\prime}\left(b_{t}\right) .
$$

Also ist auch $f$ unimodal.

\subsubsection{Deterministisches Chaos}

Nach den Ausführungen des vorherigen Unterabschnitts ist prinzipiell jede unimodale Funktion geeignet, die oben dargestellten Annahmen abzubilden. Die folgende Analyse wird am Beispiel der Funktionenfamilie

$$
b_{t+1}=f_{n}\left(b_{t}\right)=a \cdot \sqrt[n]{b_{t}^{n}\left(1-b_{t}^{n}\right)}, n \in \mathbb{R}^{+}, a>0
$$

durchgeführt. Diese Differenzengleichung entsteht durch die Verkettung von $\phi\left(b_{t}\right)=\sqrt[n]{b_{t}^{n}\left(1-b_{t}^{n}\right)}$ und $\psi\left(z_{t}\right)=a \cdot z_{t}$. Die Spezifikation von $\psi$ lässt sich so interpretieren, dass die Unternehmensleitung die Budgetzuteilung für die Folgeperiode proportional zum Zielerreichungsgrad der gegenwärtigen Periode ansetzt. Das ist zum Beispiel der Fall, wenn das Werbebudget als fester Prozentsatz des Gewinns festgelegt wird. In der gegenwärtigen Spezifikation (5.3) kann $a$ allerdings auch Werte größer als eins annehmen, ist also erst nach einer linearen Transformation als Prozentsatz interpretierbar. Darüber hinaus ist $\phi$ unimodal, so dass die getroffenen Annahmen erfüllt sind. Der genaue Verlauf hängt vom konkreten $n$ ab.

Die Entwicklung der Budgets geht wie folgt vor sich: Ausgehend von einem Startpunkt $t_{0}$, zu dem das System eingeführt wird, legt die Unternehmensleitung ein Budget fest. Die Abteilung erreicht damit gemäß $\phi$ einen 
bestimmten Zielerreichungsgrad, wobei sie sich optimal verhält und den maximalen Zielerreichungsgrad realisiert. Niedrige und hohe Budgets führen zu niedrigen Zielerreichungsgraden, mittlere Budgets zu hohen Zielerreichungsgraden. Die Unternehmensleitung nimmt den Zielerreichungsgrad zur Kenntnis und setzt gemäß $\psi$ das Budget für die nächste Periode fest, mit dem die Abteilung wiederum gemäß $\psi$ den Zielerreichungsgrad in der nächsten Periode erwirtschaftet. Abbildung 5.6 veranschaulicht den Zusammenhang noch einmal.

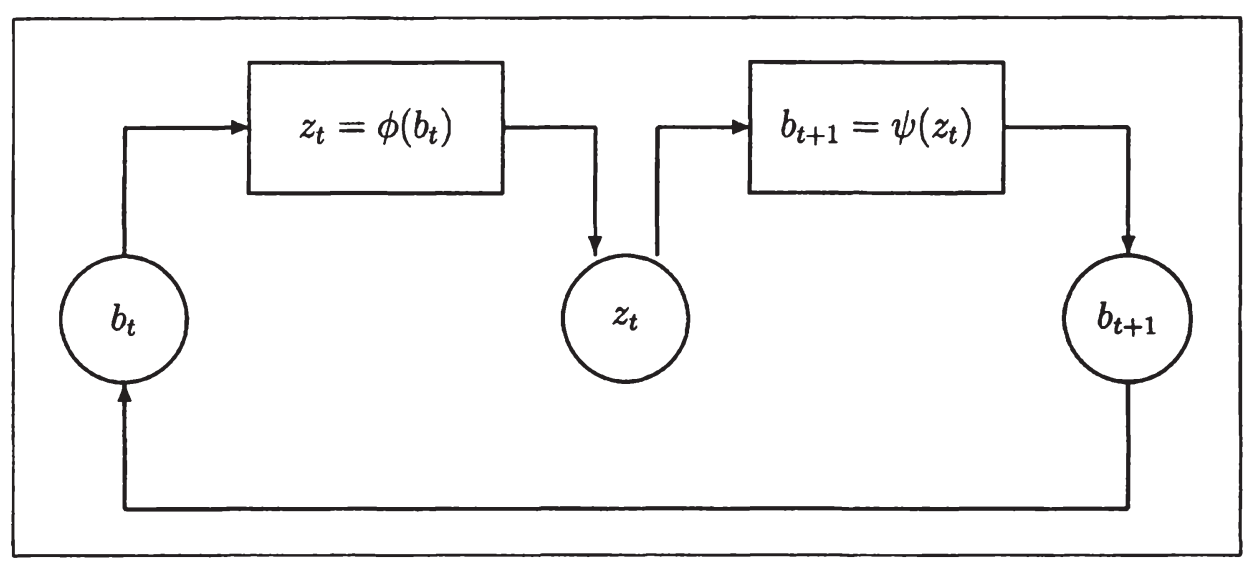

Abbildung 5.6: Regelkreis des Modells $\mathcal{B}$
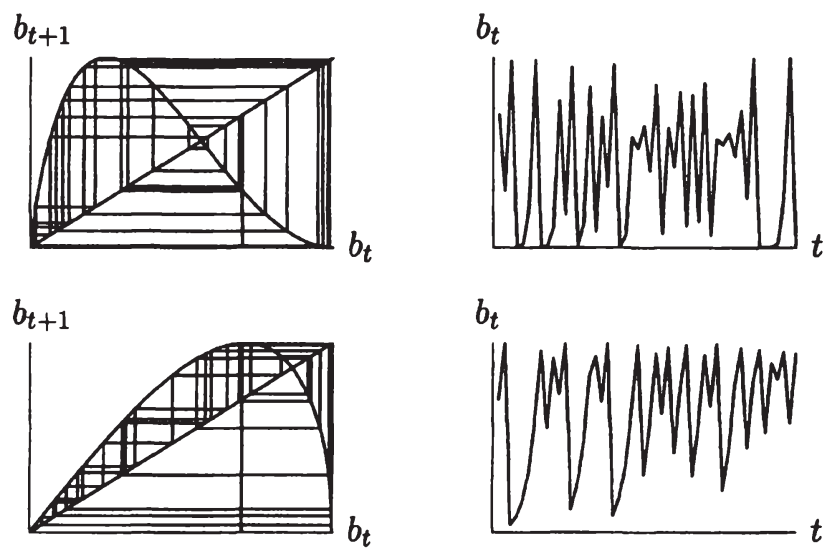

Abbildung 5.7: Phasendiagramme und Bahnen des deterministischen Budgetmodells für $n=\frac{1}{2}$ (oben) und $n=2$ (unten). 
Abbildung 5.7 zeigt zwei Phasendiagramme für $n=\frac{1}{2}$ und $n=2$ und die zugehörigen Bahnen. Die Verwandtschaft mit dem quadratischen Iterator einschließlich der Chaos-Eigenschaft ist deutlich zu erkennen, wie auch der folgende Satz bestätigt.

\section{Satz 5.5}

$f_{n}:[0,1] \rightarrow[0,1]$ ist für $a=\sqrt[n]{4}$ topologisch chaotisch im Sinne von Definition 3.7 .

Beweis. Sei $h:[0,1] \rightarrow[0,1], b_{t} \mapsto b_{t}^{n}$. $h$ ist stetig und surjektiv auf $[0,1]$. Sei außerdem $g:[0,1] \rightarrow[0,1], b_{t} \mapsto a^{n} \cdot b_{t}\left(1-b_{t}\right)$. Dann gilt:

$$
g\left(h\left(b_{t}\right)\right)=a^{n} \cdot b_{t}^{n}\left(1-b_{t}^{n}\right)=h\left(f_{n}\left(b_{t}\right)\right)
$$

d.h. $f$ und $g$ sind nach Definition 3.13 topologisch semikonjugiert. Für $a=$ $\sqrt[n]{4}$ ist $g$ nach Satz 3.9 topologisch transitiv und besitzt dichte periodische Punkte. Gemäß Satz 3.14 gilt dies auch für $f_{n}$ bei $a=\sqrt[n]{4}$. Aus Satz 3.12 folgt, dass $f_{n}$ sensitiv abhängig von den Anfangsbedingungen ist und damit chaotisch im Sinne von Definition 3.7.

Die Konstruktion der Funktionenfamilie $f_{n}$ beruht auf der Definitionsgleichung

$$
f_{n}=h_{n}^{-1} \circ g \circ h_{n}
$$

mit der als chaotisch bekannten Funktion $g$ und dem Homöomorphismus $h$. Die gefundenen Ergebnisse können in qualitativer Hinsicht auf alle weiteren Konstruktionen mit anderen Homöomorphismen verallgemeinert werden. Insofern hat die Analyse von $f_{n}$ den Charakter eines Beispiels, anhand dessen eine graphische Veranschaulichung möglich ist.

Die topologische Äquivalenz zum quadratischen Iterator spiegelt sich in den Feigenbaum-Diagrammen in Abbildung 5.8 wider. Auf den linken Seiten der Diagramme zeigt sich das Szenario der Periodenverdopplungen, das jenseits der Feigenbaum-Punkte in die chaotischen Bänder ${ }^{345}$ mündet. Auch die Fenster mit stabilen Zyklen, die die chaotischen Bereiche unterbrechen, sind zu erkennen. Die Parameterwerte, an denen Periodenverdopplungen oder Bänderspaltungen stattfinden, stehen im Verhältnis $a_{n_{1}}=a_{n_{2}}^{n_{2} / n_{1}}$. Der Vergleich der beiden Diagramme lässt erkennen, dass die Systeme über eine Potenzfunktion konjugiert sind. Beide erscheinen wie das Bifurkationsdiagramm des quadratischen Iterators, der ja den Spezialfall für $n=1$ darstellt, durch eine verzerrende Linse betrachtet.

\footnotetext{
${ }^{345}$ Es sei klar gestellt, dass die Bänder numerisch chaotisch sind, d.h. die LyapunovExponenten sind positiv. Topologisches Chaos im Sinne von Definition 3.7 gibt es nur an den rechten Rändern der Feigenbaum-Diagramme.
} 


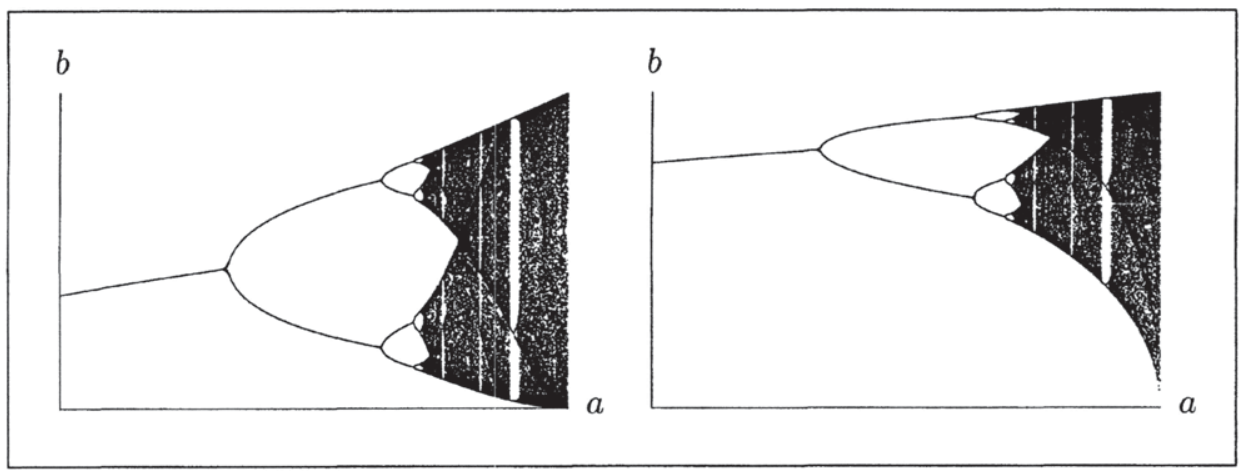

Abbildung 5.8: Feigenbaum-Diagramme des Modells $\mathcal{B}$, deterministische Variante, für $n=\frac{1}{2}$ und $n=2$.

Auch die Lyapunov-Exponenten in Abbildung 5.9 verhalten sich für verschiedene Parameterwerte $a$ in mit Abbildung 3.9 vergleichbarer Weise. Exponenten mit dem Wert null zeigen Bifurkationen an. Rechts des FeigenbaumPunktes findet man positive Exponenten, die den chaotischen Bändern entsprechen, aber auch negative Exponenten für die periodischen Fenster.
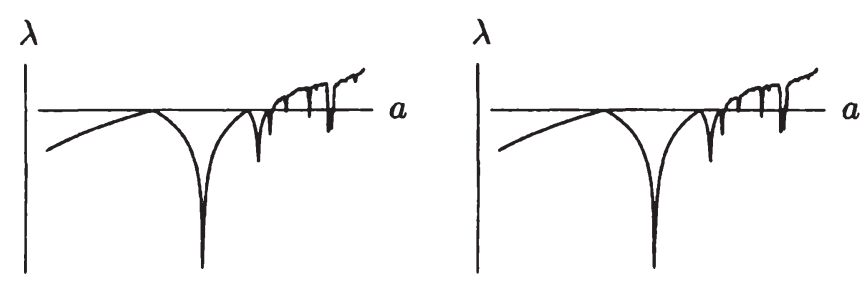

Abbildung 5.9: Lyapunov-Exponenten des Modells $\mathcal{B}$, deterministische Variante, für $n=\frac{1}{2}$ und $n=2$.

In der Analyse wurde $\psi$ als lineare Funktion mit dem Parameter $a$ aufgefaßt, also eine proportionale Beziehung zwischen Zielerreichungsgrad $z_{t}$ und Budgetzuweisung $b_{t+1}$. Der Proportionalitätsfaktor $a$ ist eine Entscheidungsvariable der Unternehmensleitung. Sie bestimmt, wieviel Prozent des Gewinns in die Werbung der Folgeperiode gesteckt werden. Wie die Abbildung 5.8 gezeigt hat, steigt mit $a$ die Komplexität des Systemverhaltens. Im Interesse der Unternehmensleitung liegt in der Regel eine möglichst gleichmäBige Entwicklung. Periodische Zyklen hoher Ordnung oder gar irreguläres Verhalten erschweren die mittel- und langfristige Planung ungemein. Mit einem hinreichend kleinen Proportionalitätsfaktor lässt sich das gewünschte 
Ergebnis erzielen; das System verharrt nach einer Einschwingphase in einem stationären Gleichgewicht, ähnlich wie es für den quadratischen Iterator in Abbildung 3.22 auf Seite 95 gezeigt wurde. Nun kann es aber auch vorkommen, dass sich die Unternehmensleitung für einen höheren Proportionalitätsfaktor entscheidet. In der Einführungsphase eines Produktes oder für einen Relaunch liegen die Ausgaben für Werbung relativ zu Umsatz oder Gewinn wesentlich höher als etwa in der Reifephase. Wenn der Zusammenhang zwischen Rückkopplung, hohem Parameterwert und irregulären Schwankungen der Unternehmensleitung nicht bekannt ist, wird sie unweigerlich die falschen Schlüsse ziehen und wirkungslose Maßnahmen ergreifen. So könnte sie etwa reagieren, indem sie den Parameterwert laufend ändert, um den Schwankungen gegenzusteuern. Dies wird das System jedoch möglicherweise zusätzlich destabilisieren.

Herrscht jedoch ein Bewusstsein für die mögliche Unvorhersagbarkeit und Irregularität nichtlinearer Systeme, kann besser zielgerichtet eingegriffen werden. Der chaosgenerierende Mechanismus besteht darin, dass die Budgetzuweisung an der Zielerreichung ausgerichtet wird, die wiederum vom Budget determiniert wird. So besteht die Problemlösung hier darin, das System zu entkoppeln, d.h. die Entscheidung für die kommende Periode so wenig wie möglich von Daten für die laufende Periode abhängig zu machen. Für die Einführungsphase eines Produktes könnte so das Werbebudget über mehrere Perioden hinweg konstant gehalten werden. Nun dürfte eine vollständige Entkopplung kaum möglich sein. Die wohl radikalste Lösung wäre, das Budget in jeder Periode nach der Methode des Zero-Base-Budgeting auszuarbeiten. ${ }^{346}$ Hierbei geht man gedanklich davon aus, dass das Unternehmen keine Vergangenheit hat, sondern baut die notwendigen Prozesse und die zugehörigen Budgets systematisch neu auf. Das Zero-Base-Budgeting dient nicht unbedingt einseitig der Kostensenkung, sondern der Reallokation der in den Gemeinkostenbereichen eingesetzten finanziellen Ressourcen und ist damit als Koordinationsinstrument grundsätzlich geeignet. ${ }^{347}$ Dieser Planungsansatz dürfte jedoch für die in jeder Periode neu zu treffenden Entscheidungen über die Budgetierung zu aufwendig und damit zu teuer sein. Ein gewisses Maß der Extrapolation vergangenheitsbezogener Daten in die Zukunft ist unvermeidlich, auch um die gesammelten Erfahrungen und Kenntnisse verwenden zu können. Um so wichtiger ist es, die Struktur der Rückkopplung zu kennen und die Parameter zu identifizieren, die die globale Dynamik bestimmen. Prinzipiell ungefährlich sind konstante oder sonstwie regelgebundene Fortschreibungen. Beispielsweise könnte man den Lebenszyklus eines Produk-

${ }^{346}$ Vgl. z.B. Maly (1982), Volz (1987) und Marettek (1982).

347 Vgl. Serfling (1992), S. 189. 
tes prognostizieren und das Werbebudget gemäß der Position des Produktes im Zyklus festlegen. Zwischen Werbebudget und Lebenszyklus besteht eine wesentlich schwächere Interdependenz als zwischen Werbebudget und Periodengewinn. Ohne Rückkopplung kann es aber auch nicht zu chaotischen Schwingungen kommen.

\subsubsection{Stochastisches Chaos}

Das Modell zur Budgetierung wird wegen seiner einfachen Struktur verwendet, um im Folgenden eine grundsätzliche Frage zu untersuchen. ${ }^{348}$ Chaotische Systeme zeigen ein stochastisch anmutendes Verhalten, obgleich ihre Bewegungsgleichungen vollkommen deterministisch sind. Am Beispiel des Budgetmodells werden nun die Auswirkungen zusätzlicher stochastischer Einflüsse untersucht. Der Systemgleichung (5.3) wird ein weißes Rauschen hinzugefügt, d.h. der grundsätzliche Zusammenhang zwischen Budgethöhe und Zielerreichungsgrad bleibt bestehen, doch es gibt weitere Einflüsse auf den Zielerreichungsgrad, bei denen der kausale Zusammenhang nicht bekannt ist.

Die Differenzengleichung lautet nun

$$
b_{t+1}=a \sqrt[n]{b_{t}^{n}\left(1-b_{t}^{n}\right)}+\epsilon_{t}
$$

mit der Zufallsvariablen $\epsilon_{t}$, für die eine Normalverteilung mit Erwartungswert $\mu=0$ und Varianz $\sigma^{2}>0$ angenommen wird: $\epsilon_{t} \sim N\left(0, \sigma^{2}\right)$.

Die Feigenbaum-Diagramme in Abbildung 5.10 zeigen die Wirkung des weißen Rauschens. Die Endzustände für kleine Parameterwerte $a$ sind keine Fixpunkte oder stabile Zyklen mehr, die sich als scharfe Linien zeigen, sondern es entstehen Streifen, die anzeigen, in welchen Bereich die Budgethöhen $b_{t}$ mit hoher Wahrscheinlichkeit fallen. Für die Erstellung der Diagramme ist eine sehr kleine Varianz verwendet worden, um die zugrunde liegende chaotische Struktur nicht zu sehr zu überlagern.

Im Bereich der chaotischen Bänder wird deutlich, dass sich das System bei gegebenem Parameter $a$ wie eine Mischung der deterministischen Systeme bei benachbarten Parametern verhält. Dies erscheint auf den ersten Blick überraschend, da sich das Rauschen annahmegemäß nicht direkt auf $a$ auswirkt. Insofern kann die Deutung von $a$ als Entscheidungsvariable der Unternehmensleitung bei der Festlegung des Budgets aufrecht erhalten werden. In der Tat lässt sich aber zeigen ${ }^{349}$, dass eine Störung der Systemvariablen $b$

348 Vgl. auch Pinkwart (1992).

${ }^{349}$ Vgl. Crutchfield, Farmer und Huberman (1982). 
ein ähnliches Verhalten hervorruft wie eine Störung des Parameters $a$ in der Form

$$
b_{t+1}=\left(a+\eta_{t}\right) \sqrt[n]{b_{t}^{n}\left(1-b_{t}^{n}\right)} \operatorname{mit} \eta \sim N(0, \sigma) .
$$

Eine einfache Umformung zeigt, dass dieses System ebenfalls einen additiven Störterm besitzt, allerdings von der Form $\frac{\eta}{a} \cdot f(b)$, d.h. der Term ist nicht normalverteilt. Die statistischen Eigenschaften beider Formen von Störtermen stimmen bei hinreichend kleiner Standardabweichung aber überein. ${ }^{350}$

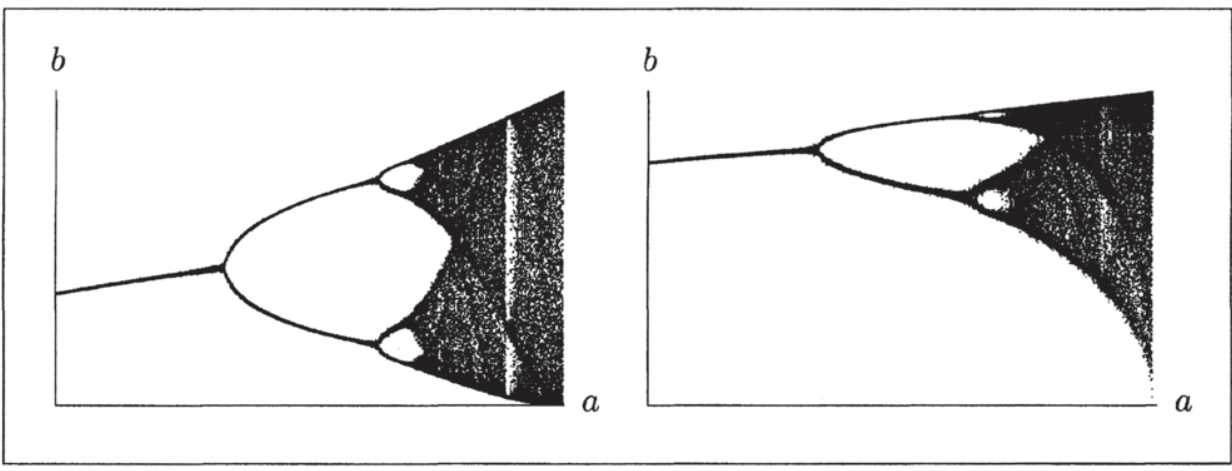

Abbildung 5.10: Feigenbaum-Diagramme des Modells $\mathcal{S}$, stochastische Variante, für $n=\frac{1}{2}$ und $n=2$.

Diese statistische Äquivalenz hat erstens zur Folge, dass es keine eindeutigen Bifurkationspunkte mehr gibt, sondern Bereiche, in denen keine Zuordnung zu Zyklen bestimmter Länge möglich ist. Dies ist unmittelbare Folge der Beobachtung, dass sich das System wie eine Mischung des deterministischen Systems links des Bifurkationspunktes mit kleinerem Zyklus und rechts des Bifurkationspunktes mit größerem Zyklus verhält. Der Entscheidungsträger steht vor dem Problem, den Übergang zwischen Zyklengrößen nicht genau erkennen zu können, so dass er sich der Bifurkation womöglich gar nicht bewusst wird.

Zweitens verschwinden Zyklen höherer Zweierpotenzen. Die Diagramme in Abbildung 5.10 lassen den Zyklus der Periode vier noch erkennen, danach folgt der Übergang in die Bänder. ${ }^{351}$ Der Weg ins Chaos ist dadurch erheblich verkürzt.

Drittens erkennt man im Fenster der Periode drei wesentlich mehr Punkte zwischen dem deterministischen Orbit als bei Zyklen in der Bifurkationskas-

350 Vgl. Kelsey (1988), S. 12.

${ }^{351}$ Solches Verhalten ist für den Übergang von laminaren zu turbulenten Flüssen empirisch bestätigt. Vgl. Kelsey (1988), S. 13. 
kade. Dies liegt daran, dass stabile Zyklen wie derjenige der Periode drei direkt benachbart mit chaotischen Bereichen sind. Der Effekt des Rauschens ist bei Zyklen der Periode drei also grundsätzlich anders als bei Zyklen mit gerader Periode, wo aus den Zyklen schmale Streifen werden, zwischen denen die Wahrscheinlichkeit beinahe bei null liegt. Ein Zyklus der Periode zwei oder vier der Zielerreichungsgrade, der bei entsprechender Periodenlänge z.B. bei oberflächlicher Betrachtung auf Saisoneinflüsse zurückgeführt werden könnte, ist bei schwachem Rauschen in der Bahn noch deutlich zu erkennen. Ein Zyklus der Periode drei oder sechs hingegen wird trotz gleich schwachem Rauschen wesentlich stärker gestört, weil das deterministische Chaos der benachbarten Parameterwerte hinzukommt.

Und viertens verschwinden wegen der Nachbarschaft zu chaotischen Bereichen die meisten periodischen Fenster innerhalb des chaotischen Bereiches völlig. Im Beispiel ist lediglich für $n=2$ das Fenster der Periode drei noch schwach zu erkennen; für $n=\frac{1}{2}$ gibt es keine Fenster mehr.

Man kann dies ebenfalls anhand der Lyapunov-Exponenten nachvollziehen, die prinzipiell auch für stochastische Systeme berechnet werden können. In Abbildung 5.11 zeigen sich nur wenige Bifurkationen, an denen die Lyapunov-Exponenten null sind in einer Umgebung negativer Exponenten. Für große Werte von $a$ tritt numerisches Chaos auf, d.h. die Exponenten sind positiv. Periodische Fenster mit stabilen Zyklen und negativen Exponenten sind kaum noch zu beobachten. Die Entwicklung der Budgets und Zielerreichungsgrade entwickelt sich für größere Bereiche als im deterministischen Fall sensitiv abhängig von den Anfangsbedingungen. Die Prognostizierbarkeit der Entwicklung und damit die Planbarkeit ist noch stärker eingeschränkt.

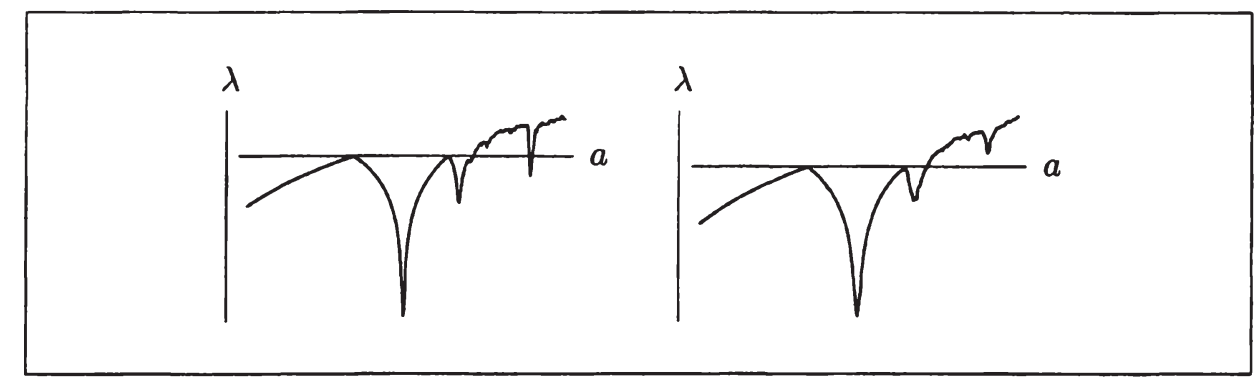

Abbildung 5.11: Lyapunov-Exponenten des Modells $\mathcal{S}$, stochastische Variante, $n=\frac{1}{2}$ und $n=2$.

Man kann also behaupten, dass chaotisches Verhalten auf externes Rauschen weniger sensitiv reagiert als periodisches stabiles Verhalten. Zyklen 
mit geringerem Existenzbereich verschwinden, nur eine endliche Zahl von Zyklen ist beobachtbar. Chaotisches Verhalten breitet sich hingegen gleichsam aus. ${ }^{352}$ Die Überlegungen zum deterministischen Fall, wie man die Auswirkungen des Chaos vermeidet, bleiben grundsätzlich gültig. Rückkopplungen sollten nach Möglichkeit vermieden werden; dort, wo sie aus Kosten- oder anderen Gründen unumgänglich sind, muss man versuchen, die Struktur des Systems so weit zu durchschauen, dass man die relevanten Parameter im stabilen Bereich halten kann.

Die für das Budgetierungsmodell gewonnenen Ergebnisse weisen über den unmittelbaren Modellrahmen hinaus. Stochastische Einflüsse sind in ökonomischen Systemen allgegenwärtig. Man mache sich dazu nur klar, welch großen Einfluss das Wetter auf die ökonomische Aktivität haben kann. ${ }^{353}$ Die Landwirtschaft ist je nach Nutzpflanze auf die unterschiedlichsten Klimate angewiesen; die Tätigkeit der Bauindustrie unterliegt starken saisonalen Schwankungen; ebenso ist der Verkehr, insbesondere See- und Luftverkehr, und damit eine Fülle logistischer Prozesse stark wetterabhängig. Eine deterministische Modellierung kann deshalb immer nur als Vereinfachung angesehen werden. Dies gilt umso mehr, wenn das Modell linear ist. Entscheidet man sich aber dafür, Nichtlinearität zuzulassen, ist chaotisches Verhalten des Systems in der Regel grundsätzlich möglich. Wir haben nun gesehen, dass topologisches Chaos nur bei ganz bestimmten Parameterkonstellationen auftritt und auch numerisches Chaos nur in engen Bereichen der Parameterwerte, die darüber hinaus von stabilen Fenstern unterbrochen werden. Schon ein sehr kleiner stochastischer Einfluss drängt aber das reguläre Systemverhalten weit zurück. Insofern besteht Anlass, das Augenmerk auf chaotische Lösungen von Systemen wie in Gleichung (5.4) zu richten. Wenn die Untersuchung stochastischer Einflüsse aus Gründen der Einfachheit im Folgenden wieder aufgegeben wird, so geschieht dies in dem Bewusstsein, dass die Bedeutung der chaotischen Regionen bei deren Vorliegen zunimmt.

\footnotetext{
352 Vgl. Kelsey (1988), S. 16.

${ }^{353}$ Man erkennt hier sehr schön, dass zufällig erscheinende Ereignisse, die in Form stochastischer Terme in Modelle integriert werden, in Wirklichkeit auf chaotischen Prozessen beruhen können.
} 


\subsubsection{Koordination durch Verrechnungspreise}

\subsubsection{Die Organisationsstruktur: Innerbetriebliche Märkte}

Entsprechend dem großen Interesse der Forschung gibt es für den Begriff des Verrechnungspreises eine Vielzahl von Definitionen. ${ }^{354}$ Nach Schneider heißen Verrechnungspreise „Preise, die bei zahlenmäßigen Abbildungen von Diensten, Sachen und Verfügungsrechten statt gegenwärtigen Marktpreisen benutzt werden, oder sie stellen Marktpreisimitationen für Dienste, Sachen und Verfügungsrechte dar, die nicht in Märkten gehandelt, sondern innerbetrieblich für Marktzufuhrhandlungen erbracht werden." ${ }^{\text {"355 }}$ Dieser Definition zufolge sind Verrechnungspreise Abweichungen von Marktpreisen, sofern letztere - bei marktfähigen Leistungen - existieren, aber aus verschiedenen Gründen nicht verwendet werden sollen oder dürfen. Als Beispiel können die Herstellungskosten dienen, mit denen Fertigerzeugnisse in Handels- und Steuerbilanzen zu bewerten sind. Eine Verwendung der Marktpreise widerspräche dem Realisationsprinzip, das aus § 252 Abs. 1 Nr. 4 HGB abgeleitet wird, und ist daher in der Handelsbilanz nicht erlaubt. Über das Maßgeblichkeitsprinzip nach $\S 5$ Abs. 1 Satz 1 EStG erstreckt sich dieses Verbot auch auf die Steuerbilanz. Im Folgenden von Interesse sind Verrechnungspreise für innerbetriebliche Leistungen, für die Marktpreise gar nicht existieren.

Die Verwendung von Verrechnungspreisen als Koordinationsmechanismus zielt darauf ab, das Modell einer effizienten Allokation von Ressourcen durch konkurrenzgleichgewichtige Märkte auf die Koordination innerhalb von Unternehmen zu übertragen. ${ }^{356}$ An diesem Bemühen ist vielfach Kritik geübt worden. Der wesentliche Punkt besteht darin, dass das Setzen korrekter Verrechnungspreise Informationen verlangt, die ein Verrechnungspreissystem überflüssig machen. Eine Unternehmensleitung, die über die erforderlichen Daten bezüglich der Grenzkosten und Grenznutzen der einzelnen Abteilungen verfügt, wäre in der Lage, gleich eine vollständige zentrale Planung durchzuführen, die zu einer optimalen Verwendung der knappen Ressourcen führt. ${ }^{357}$

Eine solche zentrale Planung ist jedoch gar nicht beabsichtigt. Wer ein Unternehmen mit Verrechnungspreisen steuern will, benötigt die für die zentrale Planung erforderlichen Informationen gar nicht; sie werden von den Abteilungen im Rahmen deren Gewinnstrebens offengelegt. Das Verhältnis zwischen

\footnotetext{
354 Vgl. Coenenberg (1999), S. 523.

355 Schneider (1997a), S. 466.

356 Vgl. Pfeiffer (1997), S. 7.

${ }^{357}$ Vgl. ausführlicher Schneider (1997a), S. $467 \mathrm{ff}$.
} 
Zentralplanung und Preissteuerung ist ein ähnliches wie zwischen Zentralverwaltungswirtschaft und Marktwirtschaft. Die Marktwirtschaft erweist sich letztlich deshalb als überlegen, weil die Informationen über Produktionsmöglichkeiten und Präferenzen der Konsumenten nicht gebündelt bei einer Zentrale vorliegen müssen. Eine Unternehmensleitung übernimmt die Aufgabe, die Verrechnungspreise solange anzupassen, bis Angebot und Nachfrage auf den innerbetrieblichen Märkten übereinstimmen, d.h. sie übernimmt die Aufgabe des Walrasianischen Auktionators ${ }^{358}$. Nach Schneider setzt dies einen Verhandlungsprozess voraus, dessen Zeitbedarf dazu führen kann, dass entweder der Verrechnungspreis überhastet und damit auf ineffizientem Niveau festgelegt wird oder Aufträge verloren gehen können. ${ }^{359}$

Hier wird gezeigt, dass unter bestimmten Bedingungen das Verhalten des Unternehmens von chaotischen Schwingungen geprägt sein kann, selbst wenn von dem Problem abgesehen wird, wie markträumende Verrechnungspreise überhaupt und rechtzeitig aufgefunden werden können.

Wir betrachten ein produzierendes System $\mathcal{V}=\{\mathcal{S}, \mathcal{D}, \mathcal{M}\}$ mit zwei Stufen, die durch einen Verrechnungspreis koordiniert werden sollen. Die vorgelagerte Stufe $\mathcal{S}$ stellt ein Zwischenprodukt in der Menge $x^{s}$ her mit Hilfe zweier Produktionsfaktoren $r$ und $\rho$, die zu den Preisen $w$ und $\omega$ beschafft werden, und liefert die Menge $x^{d}$ an die nachgelagerte Stufe $\mathcal{D}$, wo sie zusammen mit einem extern zum Preis $\nu$ beschafften Produktionsfaktor $\xi$ in ein Endprodukt $y$ eingeht, das am Absatzmarkt zum Preis $p$ verkauft wird. Zur Verrechnung dieser innerbetrieblichen Lieferung wird ein Preis $v$ verwendet, den die Unternehmensleitung $\mathcal{M}$ festsetzt. Abbildung 5.12 macht den Zusammenhang noch einmal deutlich.

Liefernde und empfangende Stelle verhalten sich beide als Preisnehmer und maximieren unabhängig voneinander ihre Gewinne. Die Unternehmensleitung übernimmt die Rolle eines Walrasianischen Auktionators ${ }^{360}$ und setzt den Verrechnungspreis so fest, dass Angebot und Nachfrage bezüglich des Zwischenproduktes ausgeglichen werden:

$$
x^{s}(v)=x^{d}(v)=x .
$$

Mit diesen Verhaltensannahmen ist grundsätzlich ein allokationseffizientes Ergebnis möglich. Die möglicherweise effizienzschädigenden Wirkungen von Preisverhandlungen in einem bilateralen Monopol zwischen Anbieter und

$\overline{358}$ Zur Erklärung der Preisanpassung auf freien Märkten wird häufig die Metapher des Walrasianischen Auktionators herangezogen. Vgl. Felderer und Homburg (1994) S. 94. 359 Vgl. Schneider (1997a), S. 470.

${ }^{360}$ Vgl. z.B. Schumann, Meyer und Ströbele (1999), S. 212. 


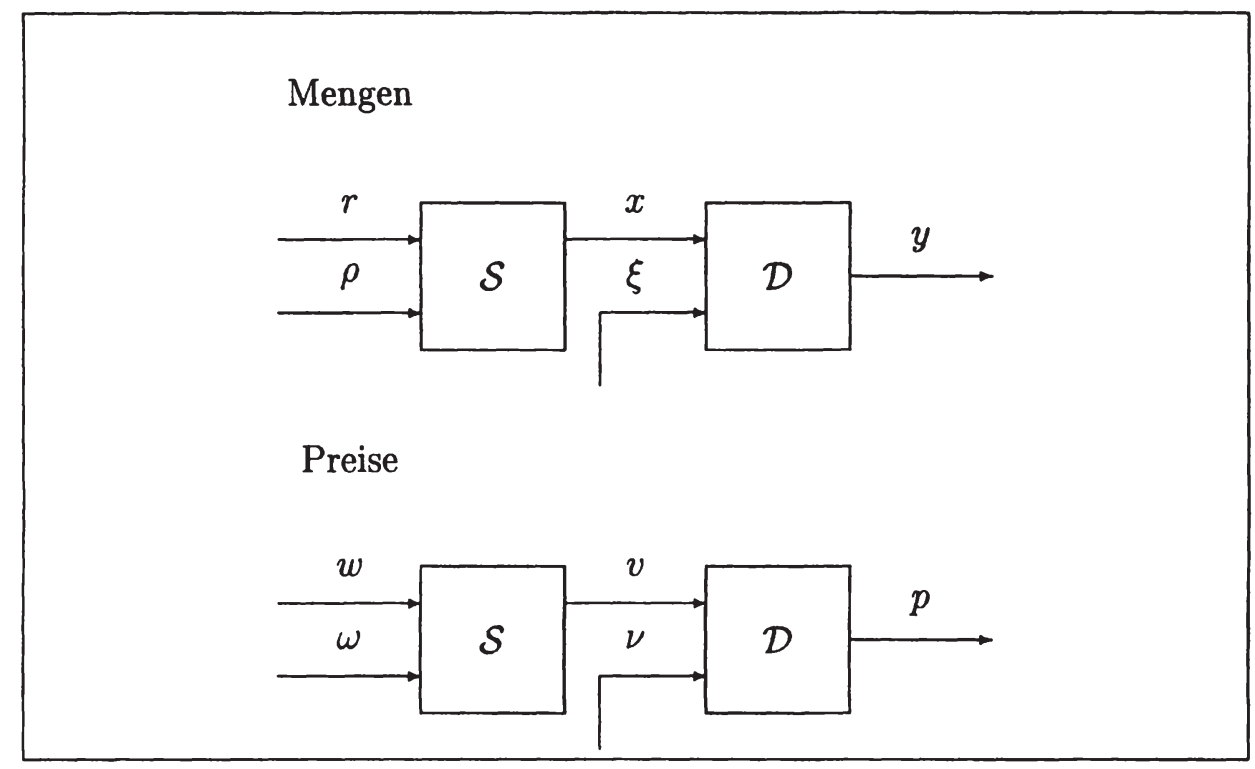

Abbildung 5.12: Struktur des produzierenden Systems $\mathcal{V}$

Nachfrager ${ }^{361}$ ohne Einflussnahme der Unternehmensleitung werden beiseite gelassen.

\subsubsection{Das formale Modell}

Im Folgenden wird zunächst für eine konkrete Technologie gezeigt, dass die Koordination von $\mathcal{V}$ über einen Verrechnungspreis in einem statischen Kontext zu einem stabilen allokationseffizienten Gleichgewicht führt. Danach wird das Modell um dynamische Eigenschaften erweitert und die Möglichkeit chaotischer Zeitpfade demonstriert. Die Technologie ist in Abbildung 5.13 dargestellt. Man erkennt, dass es sich um ertragsgesetzliche Verläufe handelt, wobei man sich bei $\mathcal{D}$ (rechte Seite) auf den Bereich $y>0$ beschränken muss.

\section{Satz 5.6}

Die Technologiemenge von $\mathcal{D}$ werde beschrieben durch

$$
T_{\mathcal{D}}=\left\{(y, x, \xi) \in \mathbb{R}_{+}^{3}: y \leq \ln (x \cdot \xi)\right\} .
$$

$\overline{361}$ Vgl. Schumann, Meyer und Ströbele (1999), S. 304ff. 

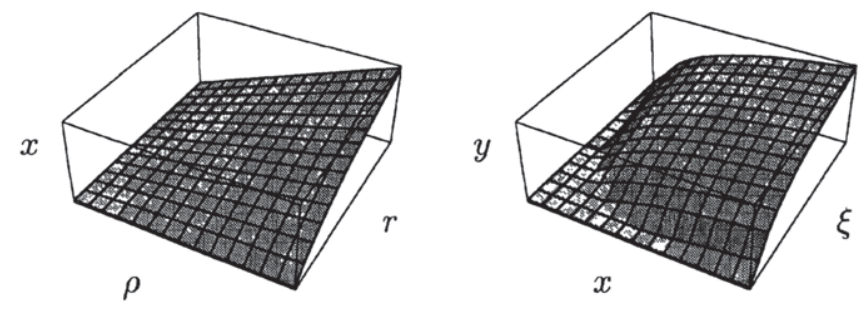

Abbildung 5.13: Technologien der liefernden (links) und der empfangenden (rechts) Stelle.

$\mathcal{D}$ sei Gewinnmaximierer und Preisnehmer auf allen Märkten ${ }^{362}$. Dann lautet die Faktornachfragefunktion für $x$

$$
x^{d}(v)=\frac{p}{v}
$$

und der Output $y$ ist positiv, wenn gilt: $v>\frac{p^{2}}{\nu}$.

Beweis. Das Gewinnmaximierungskalkül der empfangenden Stelle $\mathcal{D}$ lautet unter Berücksichtigung der Technologiemenge (5.6)

$$
\pi(p, v, \nu)=\max _{y, x, \xi} p \cdot y-v \cdot x-\nu \cdot \xi \text { s.t. } y=\ln (x \cdot \xi) .
$$

$\pi$ ist die indirekte Zielfunktion in Abhängigkeit von den Preisen.

Als Lagrange-Funktion $\mathcal{L}$ zu (5.8) erhält man

$$
\mathcal{L}(y, x, \xi, \lambda)=p \cdot y-v \cdot x-\nu \cdot \xi-\lambda(y-\ln (x \cdot \xi)) .
$$

Die notwendigen Bedingungen für (5.8) sind erfüllt, wenn alle partiellen ersten Ableitungen von $\mathcal{L}$ verschwinden ${ }^{363}$ :

$$
\begin{array}{r}
\frac{\partial \mathcal{L}}{\partial y}=p-\lambda=0, \\
\frac{\partial \mathcal{L}}{\partial x}=-v+\frac{\lambda}{x}=0, \\
\frac{\partial \mathcal{L}}{\partial \xi}=-\nu+\frac{\lambda}{\xi}=0, \\
\frac{\partial \mathcal{L}}{\partial \lambda}=-y+\ln (x \cdot \xi)=0 .
\end{array}
$$

$\overline{362}$ Die Annahme versteht sich einschließlich des innerbetrieblichen Marktes für das Zwischenprodukt $x$.

363 Vgl. Chiang (1984), S. 374. 
Hinreichend für ein Maximum von (5.8) ist die Bedingung, dass die Determinante der geränderten Hesse-Matrix $\operatorname{Hess}_{3}(\mathcal{L})$, berechnet an der durch die notwendigen Bedingungen bestimmten kritischen Stelle negativ und die Determinante des zweitgrößten Hauptminors $\mathrm{Hess}_{2}(\mathcal{L})$ positiv ist ${ }^{364}$ :

$$
\begin{gathered}
\operatorname{det}\left(\operatorname{Hess}_{3}(\mathcal{L})\right)=\operatorname{det}\left(\begin{array}{cccc}
0 & -1 & \frac{1}{x} & \frac{1}{\xi} \\
-1 & 0 & 0 & 0 \\
\frac{1}{x} & 0 & \frac{-\lambda}{x^{2}} & 0 \\
\frac{1}{\xi} & 0 & 0 & \frac{-\lambda}{\xi^{2}}
\end{array}\right)=\operatorname{det}\left(\begin{array}{ccc}
-1 & 0 & 0 \\
\frac{1}{x} & \frac{-\lambda}{x^{2}} & 0 \\
\frac{1}{\xi} & 0 & \frac{-\lambda}{\xi^{2}}
\end{array}\right) \\
=-\operatorname{det}\left(\begin{array}{cc}
\frac{-\lambda}{x^{2}} & 0 \\
0 & \frac{-\lambda}{\xi^{2}}
\end{array}\right)=-\frac{\lambda^{2}}{x^{2} \cdot \xi^{2}}<0, \\
\operatorname{det}\left(\operatorname{Hess}_{2}(\mathcal{L})\right)=\operatorname{det}\left(\begin{array}{ccc}
0 & -1 & \frac{1}{x} \\
-1 & 0 & 0 \\
\frac{1}{x} & 0 & \frac{-\lambda}{x^{2}}
\end{array}\right)=\operatorname{det}\left(\begin{array}{cc}
-1 & \frac{1}{x} \\
0 & \frac{-\lambda}{x^{2}}
\end{array}\right)=\frac{\lambda}{x^{2}}>0 .
\end{gathered}
$$

Die Determinanten haben an jeder, auch an der kritischen Stelle, die erforderlichen Vorzeichen. ${ }^{365}$

Durch Elimination von $\lambda$ aus den ersten beiden notwendigen Bedingungen (5.10) und (5.11) und Umstellen erhält man die Nachfragefunktion nach dem Produktionsfaktor $x$ :

$$
x^{d}(v)=\frac{p}{v} .
$$

Analog ergibt sich die Nachfragefunktion nach Faktor $\xi$ aus (5.10) und (5.12):

$$
\xi(\nu)=\frac{p}{\nu} .
$$

Gemäß $T_{\mathcal{D}}$ gilt $y>0$ genau dann, wenn die Ungleichung $x \cdot \xi>1$ erfüllt ist, aus den Nachfragefunktionen folgt also

$$
x \cdot \xi=\frac{p^{2}}{v \cdot \nu}>1 \Longleftrightarrow v<\frac{p^{2}}{\nu} .
$$

\section{Satz 5.7}

Die Technologiemenge der liefernden Stelle $\mathcal{S}$ sei gegeben durch

$$
T_{\mathcal{S}}=\left\{(x, r, \rho) \in \mathbb{R}_{0}^{3}: x \leq\left(\sqrt{\alpha^{2}+2 r}-\alpha\right) \rho\right\}
$$

364 Vgl. Chiang (1984), S. 385.

${ }^{365}$ Man beachte, dass gilt: $\lambda=p>0$. 
mit dem technischen Parameter $\alpha>0$; die Faktoreinsatzmenge $\rho$ sei fix. $\mathcal{S}$ sei Gewinnmaximierer und Preisnehmer auf allen Märkten ${ }^{366}$. Dann lautet die Angebotsfunktion für $x$

$$
x^{s}(v)=\frac{\rho^{2}}{w} \cdot v-\alpha \cdot \rho
$$

und das Angebot $x^{s}$ ist positiv, wenn gilt: $v>\frac{\alpha \cdot w}{\rho}$.

Beweis. $\mathcal{S}$ stellt sein Kalkül auf Basis des erwarteten Verrechnungspreises $v$ an. Wir schreiben die indirekte Gewinnfunktion von $\mathcal{S}$ entsprechend der Technologie (5.14) als

$$
\pi(v, w)=\max _{x, r} v \cdot x-w \cdot r-\omega \cdot \rho \text { s.t. } x=\left(\sqrt{\alpha^{2}+2 r}-\alpha\right) \rho .
$$

Die Lagrange-Funktion $\mathcal{L}$ zu (5.16) lautet hier

$$
\mathcal{L}(x, r, \lambda)=v \cdot x-w \cdot r-\omega \cdot \rho-\lambda\left(x-\left(\sqrt{\alpha^{2}+2 r}-\alpha\right) \rho\right) .
$$

Die notwendigen Bedingungen für (5.16) sind erfüllt, wenn

$$
\begin{array}{r}
\frac{\partial \mathcal{L}}{\partial x}=v-\lambda=0 \\
\frac{\partial \mathcal{L}}{\partial r}=-w+\lambda \frac{\rho}{\sqrt{\alpha^{2}+2 r}}=0, \\
\frac{\partial \mathcal{L}}{\partial \lambda}=-x+\left(\sqrt{\alpha^{2}+2 r}-\alpha\right) \rho=0 .
\end{array}
$$

Die Erfüllung der hinreichenden Bedingung

$$
\begin{aligned}
& \operatorname{det}\left(\operatorname{Hess}_{2}(\mathcal{L})\right)=\operatorname{det}\left(\begin{array}{ccc}
0 & -1 & \frac{\rho}{\sqrt{\alpha^{2}+2 r}} \\
-1 & 0 & 0 \\
\frac{\rho}{\sqrt{\alpha^{2}+2 r}} & 0 & \frac{-\lambda \cdot \rho}{\sqrt{\left(\alpha^{2}+2 r\right)^{3}}}
\end{array}\right) \\
& =\operatorname{det}\left(\begin{array}{cc}
-1 & 0 \\
\frac{\rho}{\sqrt{\alpha^{2}+2 r}} & \frac{-\lambda \cdot \rho}{\sqrt{\left(\alpha^{2}+2 r\right)^{3}}}
\end{array}\right)=\frac{\lambda \cdot \rho}{\sqrt{\left(\alpha^{2}+2 r\right)^{3}}}>0
\end{aligned}
$$

ist an allen Stellen sicher gestellt.

Durch Einsetzen von (5.18) in (5.19) und Umstellen erhält man die Faktornachfrage nach $r$ :

$$
r=\frac{1}{2}\left(\left(\frac{\rho \cdot v}{w}\right)^{2}-\alpha^{2}\right)
$$

${ }^{366}$ Die Annahme bezieht sich wiederum auch auf den innerbetrieblichen Markt für $x$. 
Einsetzen der Faktornachfrage in die dritte Bedingung (5.20) ergibt die Angebotsfunktion

$$
x^{s}(v)=\frac{\rho^{2}}{w} \cdot v-\alpha \cdot \rho .
$$

Das Angebot ist positiv, wenn

$$
\frac{\rho^{2}}{w} \cdot v-\alpha \cdot \rho>0 \Longleftrightarrow v>\frac{\alpha \cdot w}{\rho} .
$$

\subsubsection{Statische Eigenschaften: Allokationseffizienz}

Mit den hergeleiteten Reaktionsfunktionen (5.7) und (5.16) wird zunächst gezeigt, dass ein eindeutiges stabiles Gleichgewicht existiert, und daran anschließend, dass dieses Gleichgewicht allokationseffizient ist.

\section{Satz 5.8}

Seien das Angebot von $\mathcal{S}$ und die Nachfrage von $\mathcal{D}$ gegeben durch

$$
x^{s}(v)=\frac{\rho^{2}}{w} \cdot v-\alpha \cdot \rho \quad \text { und } \quad x^{d}(v)=\frac{p}{v} .
$$

Sei außerdem $t \in \mathbb{R}$. $\mathcal{M}$ passe $v$ in einem Tâtonnement-Prozess $h$ nach folgender Maßgabe an: ${ }^{367}$

$$
h: \mathbb{R} \rightarrow \mathbb{R}, x^{d}(v)-x^{s}(v) \mapsto \dot{v} \quad \text { mit } \quad h(0)=0, h^{\prime}>0 .
$$

Dann ist

$$
v^{*}=\frac{\alpha \cdot w+\sqrt{w\left(4 p+\alpha^{2} \cdot w\right)}}{2 \rho}
$$

das eindeutige und lokal stabile Gleichgewicht.

Beweis. Durch Gleichsetzen von Angebots- und Nachfragefunktion und Aufösen nach $v$ erhält man

$$
\frac{\rho^{2}}{w} \cdot v^{*}-\alpha \cdot \rho=\frac{p}{v^{*}} \Longleftrightarrow v^{*}=\frac{\alpha \cdot w+\sqrt{w\left(4 p+\alpha^{2} \cdot w\right)}}{2 \rho},
$$

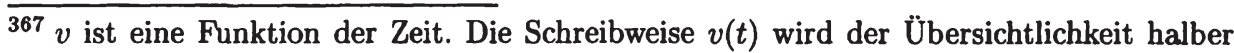
nicht verwendet. $\dot{v}$ ist die Ableitung von $v$ nach der Zeit. Die Verwendung kontinuierlicher Zeit ist lediglich eine mathematische Bequemlichkeit. 
wobei $v^{*}>0$ bereits vorausgesetzt wird.

Wegen $x^{d}\left(v^{*}\right)=x^{s}\left(v^{*}\right)$ gilt $h\left(x^{d}\left(v^{*}\right)-x^{s}\left(v^{*}\right)\right)=0$. Für $v \neq v^{*}$ ist die Differentialgleichung (5.22) ohne weitere Annahmen über $h$ nicht lösbar. Wir linearisieren $h$ durch eine Taylor-Entwicklung ${ }^{368}$ im Gleichgewicht $v^{*}$ unter Vernachlässigung des Restgliedes zweiter Ordnung ${ }^{369}$ :

$$
\begin{aligned}
& h\left(x^{d}(v)-x^{s}(v)\right) \\
& \approx h\left(x^{d}\left(v^{*}\right)-x^{s}\left(v^{*}\right)\right)+h^{\prime}\left(x^{d}\left(v^{*}\right)-x^{s}\left(v^{*}\right)\right) \frac{d\left(x^{d}\left(v^{*}\right)-x^{s}\left(v^{*}\right)\right)}{d v}\left(v-v^{*}\right) \\
& \quad=h^{\prime}\left(x^{d}\left(v^{*}\right)-x^{s}\left(v^{*}\right)\right) \frac{d\left(x^{d}\left(v^{*}\right)-x^{s}\left(v^{*}\right)\right)}{d v}\left(v-v^{*}\right) .
\end{aligned}
$$

Es gilt

$$
\frac{d\left(x^{d}\left(v^{*}\right)-x^{s}\left(v^{*}\right)\right)}{d v}=-\frac{p}{\left(v^{*}\right)^{2}}-\frac{\rho^{2}}{w}<0
$$

und mit $h^{\prime}>0$

$$
v \gtrless v^{*} \Longleftrightarrow \dot{v} \lessgtr 0 \text {. }
$$

Die Funktion $h$ beschreibt das Gesetz des Marktes, das die Unternehmensleitung umsetzt. Ihr Argument $x^{d}-x^{s}$ stellt die Überschussnachfrage dar. Ist sie positiv, steigt der Preis; ist sie negativ, sinkt er. Wenn Angebot und Nachfrage übereinstimmen, kommt die Preisbewegung zum Stillstand. Insofern ist das Gleichgewicht ein methodisches, weil es einen Ruhezustand darstellt, in dem sich die Variablen nicht mehr verändern, und zugleich auch ein theoretisches, weil der Markt geräumt ist. ${ }^{370}$ Der Ablauf dieses Tâtonnements - die Unternehmensleitung ruft solange neue Verrechnungspreise aus, bis die rückgemeldeten Angebots- und Nachfragemengen übereinstimmen geschieht nach der Logik des Modells in einem Zeitpunkt. Der Zeitverbrauch in der Realität hat Anlass zu dem Einwand gegeben, damit ließe man sich möglicherweise Chancen entgehen oder gerate unter Entscheidungsdruck. ${ }^{371}$ Dieser Einwand erscheint jedoch nicht zwingend, da beim heutigen Stand der Kommunikationstechnologie ein längerer Verhandlungsprozess nicht unabdingbare Voraussetzung für ein Zustandekommen eines Verrechnungspreises

\footnotetext{
368 Vgl. Chiang (1984), S. 256ff.

${ }^{369} \mathrm{Da}$ die Linearisierung nur in einer Umgebung von $v^{*}$ eine hinreichend genaue Näherung darstellt, gilt die Stabilitätsanalyse nur lokal. Um globale Stabilität nachzuweisen, sind weitergehende Annahmen über den Tâtonnement-Prozess nötig.

370 Vgl. zu den Gleichgewichtsbegriffen Felderer und Homburg (1994), S. 12.

371 Vgl. Schneider (1997a), S. 470.
} 
ist. Das Mitspracherecht der Abteilungen beschränkte sich ohnehin auf die Rückmeldung der gewünschten Mengen, die bei Gewinnmaximierung wahrheitsgemäß erfolgt.

\section{Satz 5.9}

Eine Setzung des Verrechnungspreises nach Gleichung (5.23) maximiert den Gesamtgewinn des Unternehmens.

Beweis. Es genügt zu zeigen, dass eine zentrale Gewinnmaximierung über die Produktionsstufen hinweg zur selben Menge des Zwischenproduktes $x$ führt wie die dezentrale Allokation über $v^{*}$.

Nach Gleichung (5.21) und (5.23) gilt bei dezentraler Allokation:

$$
x\left(v^{*}\right)=\frac{\rho}{2 w}\left(\alpha \cdot w+\sqrt{w\left(4 p+\alpha^{2} \cdot w\right)}\right)-\alpha \cdot \rho .
$$

Das zentrale Gewinnmaximierungskalkül schreibt man

$$
\pi(p, w, \nu)=\max _{y, r, \xi} p \cdot y-w \cdot r-\nu \cdot \xi \text { s.t. } y=\ln \left(\xi\left(\sqrt{\alpha^{2}+2 r}-\alpha\right) \rho\right) .
$$

Die Lagrange-Funktion $\mathcal{L}$ zu (5.29) lautet

$$
\mathcal{L}(y, r, \xi, \lambda)=p \cdot y-w \cdot r-\nu \cdot \xi-\lambda\left(y-\ln \left(\xi\left(\sqrt{\alpha^{2}+2 r}-\alpha\right) \rho\right)\right) .
$$

Die notwendigen Bedingungen für (5.29) sind erfüllt, wenn

$$
\begin{aligned}
\frac{\partial \mathcal{L}}{\partial y}=p-\lambda & =0, \\
\frac{\partial \mathcal{L}}{\partial r}=-w+\lambda \frac{\rho}{\sqrt{\alpha^{2}+2 r}\left(\sqrt{\alpha^{2}+2 r}-\alpha\right)} & =0, \\
\frac{\partial \mathcal{L}}{\partial \xi}=-\nu+\frac{\lambda}{\xi} & =0, \\
\frac{\partial \mathcal{L}}{\partial \lambda}=-y+\ln \left(\xi\left(\sqrt{\alpha^{2}+2 r}-\alpha\right) \rho\right) & =0 .
\end{aligned}
$$

Die Erfüllung der hinreichenden Bedingungen ist an allen Stellen gewährleistet:

$$
\begin{array}{r}
\operatorname{det}\left(\operatorname{Hess}_{3}(\mathcal{L})\right)=\operatorname{det}\left(\begin{array}{cccc}
0 & -1 & \frac{\partial^{2} \mathcal{L}}{\partial \lambda \partial r} & \frac{1}{\xi} \\
-1 & 0 & 0 & 0 \\
\frac{\partial^{2} \mathcal{L}}{\partial r \partial \lambda} & 0 & \frac{\partial^{2} \mathcal{L}}{\partial r^{2}} & 0 \\
\frac{1}{\xi} & 0 & 0 & \frac{-\lambda}{\xi^{2}}
\end{array}\right)=\operatorname{det}\left(\begin{array}{ccc}
-1 & 0 & 0 \\
\frac{\partial^{2} \mathcal{L}}{\partial r^{2} \lambda} & \frac{\partial^{2} \mathcal{L}}{\partial r^{2}} & 0 \\
\frac{1}{\xi} & 0 & \frac{-\lambda}{\xi^{2}}
\end{array}\right) \\
=-\operatorname{det}\left(\begin{array}{cc}
\frac{\partial^{2} \mathcal{L}}{\partial r^{2}} & 0 \\
0 & \frac{-\lambda}{\xi^{2}}
\end{array}\right)=-\frac{\partial^{2} \mathcal{L}}{\partial r^{2}} \cdot \frac{-\lambda}{\xi^{2}}<0
\end{array}
$$


mit

$$
\begin{gathered}
\frac{\partial^{2} \mathcal{L}}{\partial \lambda \partial r}=\frac{\partial^{2} \mathcal{L}}{\partial r \partial \lambda}=\frac{1}{\sqrt{\alpha^{2}+2 r}\left(\sqrt{\alpha^{2}+2 r}-\alpha\right)}>0 \\
\frac{\partial^{2} \mathcal{L}}{\partial r^{2}}=-\frac{\lambda}{\left(\alpha^{2}+2 r\right)\left(\sqrt{\alpha^{2}+2 r}-\alpha\right)^{2}}-\frac{\lambda}{\sqrt{\left(\alpha^{2}+2 r\right)^{3}}\left(\sqrt{\alpha^{2}+2 r}-\alpha\right)}<0
\end{gathered}
$$

und

$$
\operatorname{det}\left(\operatorname{Hess}_{2}(\mathcal{L})\right)=\operatorname{det}\left(\begin{array}{ccc}
0 & -1 & \frac{\partial^{2} \mathcal{L}}{\partial \lambda \partial r} \\
-1 & 0 & 0 \\
\frac{\partial^{2} \mathcal{L}}{\partial r \partial \lambda} & 0 & \frac{\partial^{2} \mathcal{L}}{\partial r^{2}}
\end{array}\right)=\operatorname{det}\left(\begin{array}{cc}
-1 & 0 \\
\frac{\partial^{2} \mathcal{L}}{\partial r \partial \lambda} & \frac{\partial^{2} \mathcal{L}}{\partial r^{2}}
\end{array}\right)=-\frac{\partial^{2} \mathcal{L}}{\partial r^{2}}>0
$$

Aus den Gleichungen (5.31) und (5.32) ergibt sich die Faktornachfrage

$$
r^{*}=\frac{2 p-\alpha^{2} \cdot w+\alpha \sqrt{w\left(4 p+\alpha^{2} \cdot w\right)}}{4 w}
$$

und daraus die optimale Menge des Zwischenprodukts $x$ :

$$
\begin{aligned}
x\left(r^{*}\right) & =\left(\sqrt{\alpha^{2}+2 \cdot \frac{2 p-\alpha^{2} \cdot w+\alpha \sqrt{w\left(4 p+\alpha^{2} \cdot w\right)}}{4 w}}-\alpha\right) \rho \\
& =\frac{\rho}{2 w} \sqrt{2 \alpha^{2} \cdot w^{2}+4 p \cdot w+2 \alpha \cdot w \sqrt{w\left(4 p+\alpha^{2} \cdot w\right)}}-\alpha \cdot \rho \\
& =\frac{\rho}{2 w}\left(\alpha \cdot w+\sqrt{w\left(4 p+\alpha^{2} \cdot w\right)}\right)-\alpha \cdot \rho \\
& =x\left(v^{*}\right) .
\end{aligned}
$$

Der Preis, der sich bei allseitigem Preisnehmerverhalten in einem von der Unternehmensleitung gesteuerten Tâtonnement einstellt, sorgt für eine effiziente Allokation der Ressourcen. Unter den Bedingungen von Satz 5.9 kann ein Verrechnungspreissystem installiert werden, das wie eine Marktallokation die dezentral vorliegenden Informationen nutzt. Die Unternehmensleitung wird von der Aufgabe einer zentralen Planung entlastet und muss lediglich den Tâtonnement-Prozess leiten. Die Effizienz der Produktionspläne wird gleichsam automatisch durch die Abteilungen sicher gestellt. Es sei allerdings noch einmal betont, dass sich dazu die Abteilungen als Preisnehmer verhalten müssen, d.h. sie dürfen über keine „Marktmacht" verfügen, die ihnen ein strategisches Verhalten ermöglicht. 


\subsubsection{Dynamische Eigenschaften: Chaotisches Tâtonnement}

Wir übertragen das Modell nun in den dynamischen Kontext mit diskreter Zeit $t \in \mathbb{Z}$. Der Verrechnungspreis wird von der Unternehmensleitung $\mathcal{M}$ für eine Periode festgesetzt, so dass auch die Planungen von liefernder und empfangender Stelle für eine Periode vorgenommen werden. Wir schreiben $v_{t}$. Der Tâtonnement-Prozess steht im Hintergrund, der innerbetriebliche Markt ist in jeder Periode geräumt. Die liefernde Stelle benötigt einen zeitlichen Vorlauf für ihre Produktion, der es ihr unmöglich macht, auf das Tâtonnement mengenmäßig zu reagieren. Sie ist dadurch gezwungen, eine Erwartung darüber zu bilden, welcher Verrechnungspreis herrschen wird, wenn die zurzeit geplante Produktionsmenge verfügbar ist, d.h. sie orientiert sich an $v_{t}^{e}$ :

$$
\forall t: \quad x_{t}^{s}\left(v_{t}^{e}\right)=x_{t}^{d}\left(v_{t}\right)
$$

Im Folgenden wird untersucht, was geschieht, wenn dieser Vorlauf nicht berücksichtigt wird, sondern die Unternehmensleitung von der Effizienz der Allokation über Verrechnungspreise überzeugt ist.

Die Erwartungen und ihre Veränderungen können exogen postuliert oder über Hypothesen endogenisiert werden. Ersteres ist theoretisch unbefriedigend und wird hier nicht weiter verfolgt. Für Letzteres werden gemeinhin drei Möglichkeiten unterschieden. ${ }^{372}$ Die einfachste Annahme ist diejenige statischer Erwartungen. Danach vermutet $\mathcal{S}$, dass der Verrechnungspreis in $t-1$ fortbestehen wird:

$$
v_{t}^{e}=v_{t-1} .
$$

Diese Form der Erwartungsbildung ist nur in einem Gleichgewicht nicht irrational. Bei über die Zeit konstantem Verrechnungspreis werden die Erwartungen nicht enttäuscht. Abseits des Gleichgewichts hingegen stimmen Erwartung und Realisation regelmäßig nicht überein, und es ist keineswegs gewiss, ob die Erwartung eine Konvergenz zum tatsächlichen Wert aufweist. Im Cobweb-Modell wird gezeigt, dass statische Erwartungen unter gewissen Bedingungen zu explodierenden Oszillationen führen können. ${ }^{373}$ Die Tatsache, dass solche Prozesse höchstens kurzfristig beobachtbar sind, dürfte darin liegen, dass Individuen aus Erwartungsfehlern lernen und langfristig von der Bildung statischer Erwartungen abgehen. ${ }^{374}$

372 Vgl. Felderer und Homburg (1994), S. 258ff.

373 Vgl. Schumann, Meyer und Ströbele (1999), S. 223ff.

${ }^{374}$ Für Cobweb-Modelle mit chaotischer Dynamik vgl. Hommes (1992, 1994). 
Der Lernprozess lässt sich durch die Annahme adaptiver Erwartungen ${ }^{375}$ modellieren:

$$
v_{t}^{e}=v_{t-1}^{e}+\theta\left(v_{t-1}-v_{t-1}^{e}\right), 0 \leq \theta \leq 1
$$

Als Basis der Erwartungsbildung wird der erwartete Verrechnungspreis der Vorperiode zugrunde gelegt und um den Erwartungsfehler multipliziert mit dem Adaptionsparameter $\theta$ korrigiert. Allgemein liegt adaptiven Erwartungen die Vorstellung zugrunde, dass die Korrektur der Erwartung um so größer ist, je größer der Erwartungsfehler in der Vorperiode war. Gleichung (5.36) trifft die übliche Annahme der Proportionalität von Korrektur und Fehler. Als Spezialfälle sind die statischen Erwartungen $(\theta=1)$ und die konstanten Erwartungen $^{376}(\theta=0)$ enthalten. Bei einem über die Zeit konstanten Verrechnungspreis konvergieren die Erwartungen gegen den tatsächlichen Wert $v_{t}=$ const.

Über das Lernmodell (5.36) kann man durch die Annahme rationaler Erwartungen noch hinaus gehen. Danach wird als erwarteter Verrechnungspreis der statistische Erwartungswert $\mathcal{E}$ verwendet, wie er sich aus der Modellstruktur und den verfügbaren Informationen ergibt:

$$
v_{t}^{e}=\mathcal{E}\left(v_{t}\right) \text {. }
$$

Eine rationale Erwartungsbildung lässt sich für Märkte durchaus rechtfertigen. Es existiert bei den Marktteilnehmern Wissen über den Preisbildungsprozess und die relevanten Einflussfaktoren, das genutzt werden kann. Dabei ist es nicht notwendig, dass jeder Anbieter und Nachfrager die notwendigen Berechnungen selbst durchführt, sondern er kann die wissenschaftlich fundierten Prognosen von Verbänden und Instituten nutzen. Im Durchschnitt wird man von rationalen Erwartungen nicht enttäuscht. ${ }^{377}$ Für das Verrechnungspreismodell ist es allerdings nicht konsistent, von rationalen Erwartungen auszugehen. Die Einführung eines Verrechnungspreissystems macht nur Sinn, wenn private Informationen, d.h. Informationen, die nur in bestimmten Abteilungen vorhanden sind, existieren und genutzt werden sollen. Dann steht aber keiner Abteilung ein Modell zur Verfügung, auf dessen Grundlage eine rationale Erwartung gebildet werden könnte. So kennt die liefernde

375 Vgl. Crawford (1993a,b).

${ }^{376}$ Konstante Erwartungen sind exogen gegeben. Thre Veränderungen können nicht aus dem Modell abgeleitet werden. Insofern sind sie lediglich Gegenstand komparativstatischer Betrachtungen.

${ }^{377}$ In Analogie dazu muss man auch kein Meteorologe sein, um eine Erwartung über das Wetter von morgen zu bilden. Es genügt den Wetterbericht zu hören. Andererseits wissen wir, dass das Wetter unter gewissen Bedingungen chaotisch ist. In einem inhärent chaotischen System nutzt also auch rationale Erwartungsbildung nichts. 
Stelle $\mathcal{S}$ die Nachfragefunktion der empfangenden Stelle $\mathcal{D}$ nicht und vice versa. Auch die Unternehmensleitung $\mathcal{M}$ kennt die Reaktionsfunktionen der Stellen nicht. Es ist gerade der Reiz eines Preissystems, dass solche Informationen nicht zentral verfügbar sein müssen, sondern durch Angebot und Nachfrage der Abteilungen offen gelegt werden. Kennte hingegen eine Stelle das Gesamtmodell, könnte sie eine zentrale Planung durchführen; die Verrechnungspreise wären überflüssig. Folglich wird im Weiteren für die liefernde Stelle $\mathcal{S}$ die Bildung adaptiver Erwartungen unterstellt, da dies weder unnötig geringe Anforderungen an die Rationalität der Entscheidungsträger impliziert wie statische oder konstante Erwartungen noch die Verfügbarkeit von Daten erfordert, die ein Verrechnungspreissystem unnötig machen würden.

\section{Satz 5.10}

Seien die Reaktionsfunktionen gegeben durch (5.7) und (5.15). Aus der Preissetzungshypothese (5.5) in Verbindung mit der Erwartungsbildungshypothese (5.36) ergibt sich die Bewegungsgleichung des Modells

$$
v_{t}=\frac{p \cdot v_{t-1}}{(1-\theta) p-\theta \cdot w \cdot \alpha \cdot \rho \cdot v_{t-1}+\theta \cdot \frac{\rho^{2}}{w} \cdot v_{t-1}^{2}} .
$$

Beweis. Wir setzen (5.15) und (5.7) in (5.5) ein und lösen nach $v_{t}^{e}$ auf:

$$
\frac{\rho^{2}}{w} \cdot v_{t}^{e}-\alpha \cdot \rho=\frac{p}{v_{t}} \Longleftrightarrow v_{t}^{e}=\frac{w \cdot p}{\rho^{2} \cdot v_{t}}+\frac{w \cdot \alpha}{\rho}
$$

Analog kann man die Gleichung für $v_{t-1}^{e}$ schreiben. Wir setzen ein in (5.36), lösen nach $v_{t}$ auf und erhalten Gleichung (5.37). ${ }^{378}$

\section{Satz 5.11}

Die Differenzengleichung (5.37) ist unimodal mit dem Modus

$$
v_{t-1}^{\max }=\sqrt{\frac{p(1-\theta)}{\frac{\rho^{2}}{w} \cdot \theta}} .
$$

Beweis. Durch Ableiten und Nullsetzen von (5.37) erhält man

$$
\frac{p \cdot w\left(p \cdot w(1-\theta)-\theta \cdot \rho^{2} \cdot v_{t-1}^{2}\right)}{\left(\theta \cdot \rho \cdot v_{t-1}\left(w \cdot \alpha-\rho \cdot v_{t-1}\right)-(1-\theta) p \cdot w\right)^{2}}=0 \Longleftrightarrow v_{t-1}^{\max }=\sqrt{\frac{p(1-\theta)}{\frac{\rho^{2}}{w} \cdot \theta}} .
$$

378 In einem volkswirtschaftlichen Modell zur Analyse von Agrarmärkten verwenden Finkenstädt und Kuhbier (1992) eine ähnliche Dynamik. 
Wir berechnen das Vorzeichen der Ableitung $v_{t}^{\prime}$ an der Stelle $(1+\epsilon) v_{t-1}^{\max }$. Es genügt die Bestimmung des Zählers, da der Nenner immer positiv ist:

$$
\begin{aligned}
\operatorname{sign} v_{t}^{\prime}= & \operatorname{sign}\left(p \cdot w\left(p \cdot w(1-\theta)-\theta \cdot \rho^{2} \cdot \frac{p(1-\theta)}{\frac{\rho^{2}}{w} \cdot \theta}\right)\right) \\
& =\operatorname{sign}\left(p^{2} \cdot w^{2}(1-\theta)-p^{2} \cdot w^{2}(1-\theta) \frac{\theta \cdot \rho^{2}}{\theta \cdot \rho^{2}}(1+\epsilon)\right)=-\operatorname{sign} \epsilon .
\end{aligned}
$$

$v_{t}$ ist links von $v_{t}^{\max }$ streng monoton steigend, rechts davon streng monoton fallend. Damit ist $v_{t}^{\max }$ der eindeutige Modus.

Um positive Verrechnungspreise sicher zu stellen, müssen wir an den Adaptionsparameter $\theta$ folgende hinreichende Bedingung stellen:

\section{Satz 5.12}

$v_{t}$ ist positiv für alle $t \in \mathbb{Z}$, wenn $\theta$ die Ungleichung

$$
\theta<\frac{4 p}{4 p+w^{3} \cdot \alpha^{2}}
$$

erfüllt.

Beweis. Es genügt zu untersuchen, wann der Nenner der Bewegungsgleichung (5.37) positiv ist. Der Zähler ist immer positiv. Es gilt:

$$
\begin{aligned}
& (1-\theta) p-\theta \cdot w \cdot \alpha \cdot \rho \cdot v_{t-1}+\theta \cdot \frac{\rho^{2}}{w} \cdot v_{t-1}^{2}>0 \\
& \Longleftrightarrow \theta\left(-p-w \cdot \alpha \cdot \rho \cdot v_{t-1}+\frac{\rho^{2}}{w} \cdot v_{t-1}^{2}\right)+p>0 .
\end{aligned}
$$

Wir schätzen die Klammer nach unten $a b$, indem wir ihren Minimalwert berechnen. Durch Differenzieren erhalten wir $v_{t-1}^{\min }=\frac{w^{2} \cdot \alpha}{2 \rho}$. Einsetzen ergibt $^{379}$ :

$$
\theta\left(-p-\frac{1}{4} w^{3} \cdot \alpha^{2}\right)+p>0 \Longleftrightarrow \theta<\frac{p}{p+\frac{1}{4} w^{3} \cdot \alpha^{2}} .
$$

Für die weiteren Betrachtungen wird nun ein numerisches Beispiel herangezogen. Wir spezifizieren die Parameter wie folgt:

$$
p=2, w=1, \alpha=1, \rho=0,25 .
$$

${ }^{379}$ Das Ungleichheitszeichen dreht sich wegen $p>0$ und damit $(\cdot)<0$ um. 
Gegenstand der Analyse ist das Systemverhalten für alternative Adaptionsparameter. Natürlich ließe sich das System beispielsweise auch für alternative Absatz- und Beschaffungspreise untersuchen. Hier soll jedoch demonstriert werden, wie das System $\mathcal{V}$ aus sich selbst heraus bei statischer Umwelt chaotische Schwingungen entwickeln kann.

Die Bewegungsgleichung heißt mit den oben angebenen Parameterwerten:

$$
v_{t}=\frac{32 v_{t-1}}{32(1-\theta)-4 \theta \cdot v_{t-1}+\theta \cdot v_{t-1}^{2}} .
$$

Die Bedingung für positive Verrechnungspreise gemäß Gleichung (5.39) lautet $\theta<\frac{8}{9}$. Ein analytischer Nachweis der Chaoseigenschaft von (5.40) wird an dieser Stelle nicht versucht. Die Bewegungsgleichung unterscheidet sich von den bisher behandelten Systemen dadurch, dass sie keinen begrenzten Definitionsbereich aufweist. Die Funktionswerte konvergieren für wachsende Argumentwerte gegen die Abszisse. Darüber hinaus geht, wie sich leicht nachrechnen lässt, der Modus für $\theta \rightarrow \frac{8}{9}$ gegen unendlich. Abbildung 5.14 zeigt ein Phasendiagramm mit zugehöriger Bahn aus dem chaotischen Bereich der Bewegungsgleichung (5.40). Man erkennt, dass für $t>0$ alle Werte $v_{t}$ zwischen Null und dem Modus liegen, auch wenn der Startwert $v_{0}$ größer als der Modus ist.

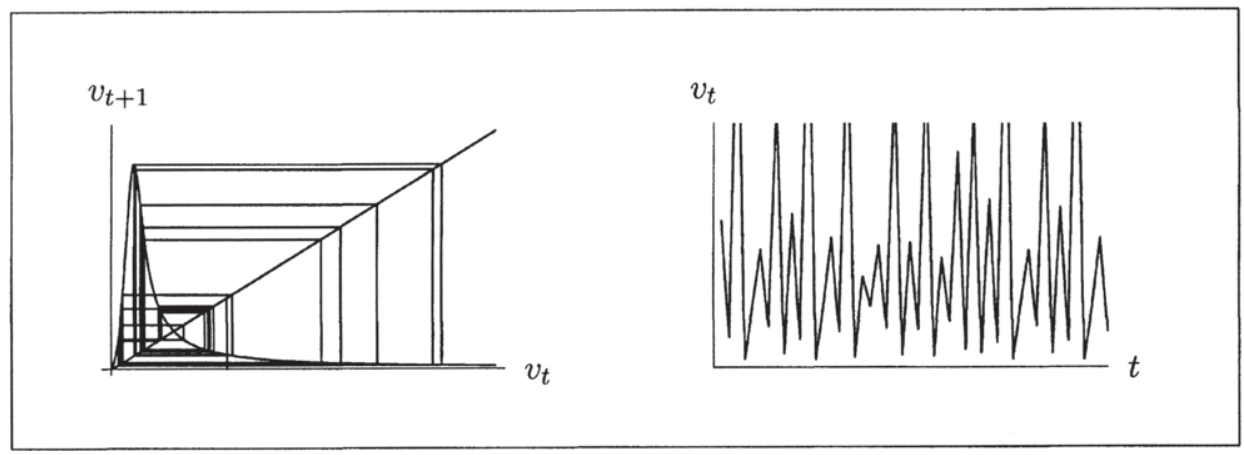

Abbildung 5.14: Phasendiagramm und Bahn des Modells $\mathcal{V}$ für $\theta=0,85$

Die Analyse der Lyapunov-Exponenten zeigt ein ähnliches Bild wie beim quadratischen Iterator in Abbildung 3.9. Man sieht in Abbildung 5.15 unten, dass für kleine $\theta$-Werte die Lyapunov-Exponenten negativ, aber zunehmend sind, bis der Wert null erreicht wird. Dies ist ein Zeichen für eine Bifurkation, was das Feigenbaum-Diagramm im oberen Teil von Abbildung 5.15 bestätigt. Dort erkennt man, dass zum selben $\theta$-Wert der stabile Fixpunkt in einen zweiperiodigen Zyklus übergeht. Dessen Stabilität lässt sich daran ablesen, 
dass die Lyapunov-Exponenten für diesen Bereich wiederum negativ werden. Auch der zweiperiodige Zyklus wird, wie alle folgenden Zyklen der Ordnung $2^{n}$ instabil, wobei die Stelle der Bifurkation einen Lyapunov-Exponenten von null besitzt.
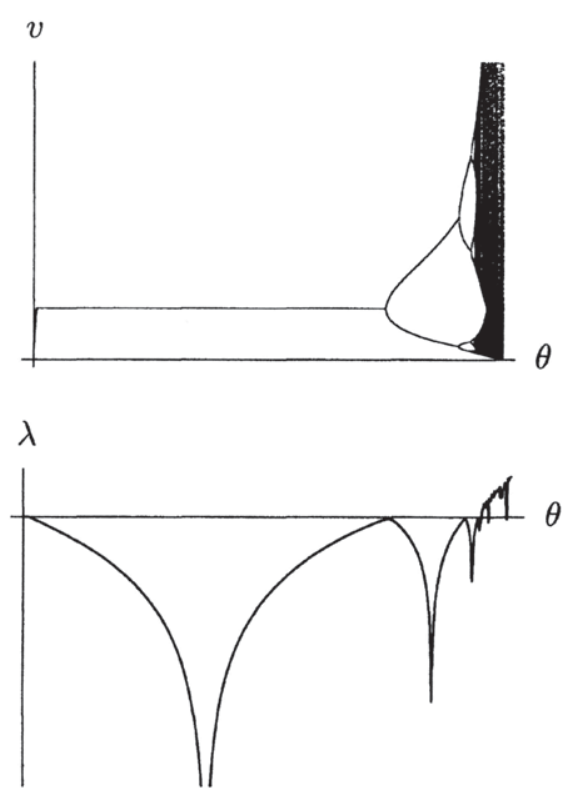

Abbildung 5.15: Feigenbaum-Diagramm und Lyapunov-Exponenten des Modells $\mathcal{V}$ für $0 \leq \theta \leq \frac{8}{9}$

Die Bifurkationen folgen immer rascher aufeinander, bis die LyapunovExponenten schließlich positiv werden. Im Feigenbaum-Diagramm lassen sich nun die typischen Bänder beobachten, die von periodischen Fenstern durchbrochen werden. Abbildung 5.16 zeigt eine Ausschnittvergrößerung des Verlaufs der Lyapunov-Exponenten und des Feigenbaum-Diagramms. Es zeigt sich wie erwartet, dass in den periodischen Fenstern, die allesamt keine Zweierpotenz als Periodenordnung besitzen, die Lyapunov-Exponenten negativ sind. Der Unterschied zu den bisher behandelten Systemen besteht darin, dass die Maxima der Bewegungsgleichung und damit auch der Orbits für steigendes $\theta$ über alle Grenzen wachsen.

\section{Hypothese 5.13}

Das System erreicht für $\theta<0,7$ den optimalen Verrechnungspreis. Ab $\theta \approx 0,7$ entsteht eine Periodenverdopplungskaskade, die bei $\theta \approx 0,83 \mathrm{zu}$ numerischem Chaos wird. 

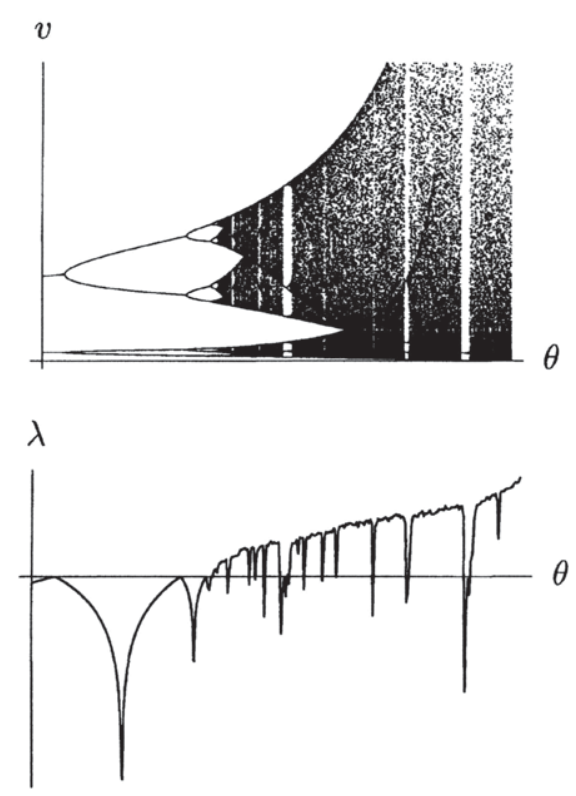

Abbildung 5.16: Feigenbaum-Diagramm und Lyapunov-Exponenten des Modells $\mathcal{V}$ für $0,8 \leq \theta \leq \frac{8}{9}$

Die Reaktionsfunktionen des vorgestellten Modells beruhen prinzipiell auf Optimierungskalkülen der liefernden und empfangenden Stelle. Die Allokationseffizienz wurde für den statischen Kontext gezeigt. Der von der Unternehmensleitung nicht berücksichtigte Produktionslag der liefernden Stelle führt jedoch in Verbindung mit der Bildung adaptiver Preiserwartungen dazu, dass der Adaptionsparameter das qualitative Systemverhalten bestimmt. Der Adaptionsparameter unterscheidet sich in gewisser Hinsicht fundamental vom chaosgenerierenden Mechanismus des Budgetmodells aus Abschnitt 5.2.1. Obzwar es sich in beiden Fällen, bei $\theta$ in Gleichung (5.37) wie bei $a$ in Gleichung (5.3), um Entscheidungsvariablen handelt, so wird doch $a$ bewusst gewählt und ist allen bekannt. Die Wahl seines $\theta$ dürfte der Entscheidungsträger hingegen nicht explizit treffen. Das Systemverhalten kann also in unterschiedliche Regionen geraten, ohne dass irgend jemand, einschließlich des Verantwortlichen selbst, die Ursache nachvollziehen könnte. Dementsprechend würden Korrekturen unweigerlich an der falschen Stelle ansetzen. Alternativ kann man unterstellen, dass der Adaptionsparameter für jedes Individuum gegeben, zwischen den Individuen aber verschieden ist. In diesem Szenario könnte der personelle Wechsel der Entscheidungskompe- 
tenz den Übergang ins Chaos verursachen. Das grundlegende Problem liegt auch in diesem System darin, dass die Ursache solcher qualitativer Änderungen nicht wahrgenommen wird. Die Unternehmensleitung geht dadurch fehl, dass sie glaubt, die Voraussetzungen für Allokationseffizienz geschaffen zu haben. Das Modell des vollkommenen Marktes, das ihr als Leitbild dient, ist aber grundsätzlich statischer Natur. Die Übertragung in den dynamischen Kontext gelingt nur unter zusätzlichen Voraussetzungen, die aufgrund der gegebenen Technologie nicht vorliegen. Hier besteht die Lösung darin, den Lag in der Produktion des Zwischenproduktes $x$ zu berücksichtigen, d.h. nicht blind darauf zu vertrauen, dass die Effizienzeigenschaft des „vollkommenen" innerbetrieblichen Marktes auch unter diesen Bedingungen noch besteht.

Abschließend soll noch darauf eingegangen werden, wie die Reaktionsfunktionen zustande kommen. Man mag einwenden, dass die zugrunde liegenden Technologien sehr speziell sind, insbesondere die Technologiemenge $T_{\mathcal{S}}$ der liefernden Stelle hat eine ungewöhnliche Gestalt. Nun lässt sich darauf erwidern, dass die postulierten Technologien mit den üblichen Annahmen vereinbar sind. Abbildung 5.13 veranschaulicht, dass die Produktionsfunktionen durchaus gewöhnliche Gestalt besitzen. Um affine bzw. hyperbolische Reaktionsfunktionen zu erhalten, müssen bei strikt rationaler Gewinnmaximierung eben solche Technologien vorliegen. Ohne die Annahme der Gewinnmaximierung sind die Funktionen (5.7) und (5.15) darüber hinaus aber auch als Heuristiken plausibel. Die affine Angebotsfunktion hat die Eigenschaft, auf dieselbe absolute Preisänderung unabhängig vom Ausgangspreis mit derselben konstanten Mengenänderung zu antworten. Die Nachfragefunktion hält die „Ausgaben" $v_{t} \cdot x^{d}$ konstant. Aufgrund dieser Eigenschaften werden solche Funktionen häufig verwendet, wenn auf eine explizite entscheidungslogische Fundierung verzichtet wird.

\subsubsection{Koordination durch Selbstabstimmung}

\subsubsection{Das Szenario}

In den vorherigen Modellen konnte der chaosgenerierende Mechanismus darin gesehen werden, dass die Unternehmensleitung als übergeordnete Instanz fehlerhafte Koordinationsregeln installierte. Das Modell zur Budgetierung bildete vertikale Koordination ab, da die untergeordnete Abteilung bei der Zuteilung des Budgets keinerlei Mitgestaltungsrecht besaß. Die Koordination über Verrechnungspreise versuchte zwar, den Marktprozess nachzuahmen, und enthält somit ein horizontales Element, doch das Problem bestand in 
dem von der Unternehmensleitung gesteckten Rahmen, der den Produktionslag der liefernden Stelle nicht berücksichtigte.

In diesem Abschnitt wird auf die Einschaltung einer übergeordneten Instanz verzichtet. Die Beteiligten stehen auf derselben hierarchischen Ebene und werden im Weiteren als Partner bezeichnet. Eine solche Situation besteht oft bei einer Kooperation von Unternehmen, etwa in Form einer Strategischen Allianz. Damit wollen die Partner Marktchancen erschließen, die einem der beteiligten Unternehmen allein aus Gründen mangelnder Kapazität oder fehlendem Know-how verschlossen blieben. Wir gehen von folgendem Szenario aus: Zwei Unternehmen stellen gemeinsam in Kooperation ein Gut in marktorientierter Produktion her. Ob es sich um die gemeinsame Herstellung eines Produktes handelt, bei dem die Partner jeweils Bauteile beisteuern, oder ob sie zueinander komplementäre Güter anbieten, die der Kunde gemeinsam konsumiert, kann offen bleiben, da beide Fälle identisch strukturiert sind.

Die nahe liegende Form der Abstimmung ist die Bildung eines unternehmensübergreifenden Koordinationsteams, das einen gemeinsamen, für beide Partner bindenden Wirtschaftsplan für die nächste Planungsperiode aufstellt. Eine rollierende Planung ist selbstverständlich denkbar. Diese Koordinationsform hat sicherlich ihre Vorzüge, die am Ende dieses Abschnitts noch einmal aufgegriffen werden. Sie ist jedoch auch mit verschiedenen Problemen behaftet.

Zunächst kann es sich als schwierig erweisen, die Machtverhältnisse im Team auszubalancieren. Mit der Aufgabe, den Wirtschaftsplan beider Partner, soweit die Kooperation betroffen ist, auszuarbeiten, besitzt das Team faktisch einen großen Einfluss auf beide Geschäftspolitiken und damit auch die entsprechende Verantwortung. Wenn die Kooperation einen gewissen Anteil am Geschäftsumfang eines oder beider Partner hat, können Fehlentscheidungen fatale Auswirkungen bis hin zum Konkurs haben. Ähnliches kann eintreten, wenn es einem Partner gelingt, seinen Einfluss im Koordinationsteam zum Schaden des anderen geltend zu machen. Entweder zerbricht die Kooperation, was beiden zum Schaden gereicht, oder der schwächere Partner wird in der Kooperation geknebelt. Nur wenn beide Partner annähernd gleiche Macht besitzen, kann man überhaupt mit einem fairen Koordinationsprozess rechnen, der beide gemäß ihrem Anteil am Marktergebnis entlohnt. Doch auch dann bleiben die Probleme bestehen, die in der Verfügbarkeit der notwendigen Informationen und in den Fähigkeiten der Teammitglieder, die vorhandenen Informationen adäquat zu verarbeiten, begründet liegen.

Beide Partner sind sich bewusst, dass der jeweils andere ein rechtlich und - soweit Geschäftstätigkeit außerhalb der Kooperation existiert - wirtschaft- 
lich selbständiges Unternehmen ist und daher eigene Interessen besitzt und verfolgt, die mit den Interessen des Partners in Konflikt stehen können. Schon deshalb, weil sie auf demselben Markt operieren, sind konfliktäre Ziele wahrscheinlich. So müssen beide jederzeit damit rechnen, dass die Kooperation aufgelöst wird, und sie mit neuen Partnern nun in Wettbewerb zueinander stehen. Vor diesem Hintergrund werden sie relevante Informationen, die ihre wahre Leistungsfähigkeit betreffen, nicht ohne Weiteres offenlegen. Damit ist die Informationsbasis als notwendige Voraussetzung einer sinnvollen Planung von fragwürdiger Qualität.

Ebenso fragwürdig wird unter den genannten Umständen, ob eine wirksame Kontrolle der Einhaltung des Wirtschaftsplans durchführbar ist. Die benötigten Kontrollinstrumente einschließlich einer Vereinbarung über anzuwendende Sanktionsmechanismen müssen zunächst institutionalisiert werden. Gerade in der Anfangsphase der Kooperation oder wenn der Zeithorizont der Zusammenarbeit unklar ist und eher kurz eingeschätzt wird, wird auf die Bereitstellung der Instrumente in der Regel verzichtet. Zum anderen erfordert die Kontrolle Informationen über Produktionskennziffern und Kosten, die aber nur von den Partnern auf freiwilliger Basis zur Verfügung gestellt werden können. Außerdem beinhalten einfache Anreizschemata in der Regel einen Anreiz, solche Daten zu verfälschen. Einigt man sich z.B. darauf, den Gesamterlös nach Abzug der Kosten beider Partner zu teilen - womöglich auch mit entsprechender Gewichtung, um eine ungleiche Bedeutung der Partner für das Marktergebnis zu würdigen - besteht ein Anreiz, die Produktionskosten zu übertreiben. Dieses Phänomen ist von öffentlichen Aufträgen her bekannt, die auf Basis einer Vollkostenkalkulation nach den Leitsätzen für die Preisermittlung (LSP) entlohnt werden. ${ }^{380}$ Im Kontext kurzfristiger Unternehmensverbünde wird in der Regel gegenseitiges Vertrauen als Voraussetzung erfolgreicher Zusammenarbeit betont. ${ }^{381}$ Solche Verbünde wie z.B. virtuelle Unternehmen sind Musterbeispiele dafür, dass die Institutionalisierung von Kontrollmechanismen ökonomisch nicht immer sinnvoll ist und daher bisweilen gar nicht angestrebt wird. Besteht ein funktionierendes Vertrauensverhältnis zwischen den Partnern, sind Kontrollen der Einhaltung der vereinbarten Wirtschaftspläne in der Tat überflüssig. In der Realität wird Vertrauen als Instrument der Vereinfachung der Geschäftsbeziehung durchaus genutzt. Jedoch liegt es bei genauerem Hinsehen im Eigeninteresse der Beteiligten, dem in sie gesetzten Vertrauen gerecht zu werden. Bei Berücksichtigung der Existenz von widerstrebenden Partikularinteressen erscheint

380 Vgl. Coenenberg (1999), S. 132ff.

381 Vgl. z.B. Byrne (1993), Davidow und Malone (1992) und kritisch Weibler und Deeg (1998). 
die Funktionsfähigkeit der Kooperation jedoch fragwürdig. ${ }^{382}$ Vertrauen als conditio sine qua non einer Kooperation erscheint nicht als tragfähige Basis. Das Rekurrieren auf eine Vertrauensbasis als Grundlage der Kooperation dient in der Regel dazu, das Problem widerstrebender Interessen wegzudefinieren, statt es zu lösen.

Schließlich stellt sich die Frage nach der Flexibilität der gemeinsamen Planung. In einer dynamischen Umwelt besteht die ständige Notwendigkeit, die Planung im Hinblick auf geänderte Parameter zu korrigieren. Ein Unternehmen, dem diese Fähigkeit fehlt, kann allenfalls in einer statischen Umwelt, die keine Anpassung erfordert, überleben. Eine Unternehmenskooperation sieht sich in diesem Zusammenhang zwei Trade-offs ausgesetzt. Fixiert man im Rahmen des Koordinationsteams den gemeinsamen Wirtschaftsplan so detailliert wie möglich, geht die Fähigkeit der flexiblen Anpassung weitgehend verloren. Es ist nur für theoretische Szenarien denkbar, dass es gelingt, alle möglichen Entwicklungen der künftigen Planungsperiode vorwegzunehmen und dafür geeignete Handlungsanweisungen festzulegen. Erfolgt hingegen nur eine Grobplanung, so fehlen für das operative Geschäft die für eine erfolgreiche Koordination wichtigen Detailinformationen; darüber hinaus lässt sich dem Plan nicht entnehmen, wie auf unvorhergesehene Ereignisse zu reagieren ist. Der gemeinsame Wirtschaftsplan ist weitgehend nutzlos.

Man könnte diesem Trade-off begegnen, indem man die Länge der Planungsperiode verkürzt und die lange Frist durch rollierende Planung erfasst. Auf diese Weise lässt sich die zukünftige Entwicklung mit geringerer Unsicherheit prognostizieren, der Plan würde seltener durch Lückenhaftigkeit versagen. Man gerät jedoch in einen zweiten Trade-off, denn mit der Planungshäufigkeit steigen auf der anderen Seite die Kosten für das Koordinationsteam. Hoch qualifizierte Angestellte mit entsprechenden Gehältern müssen von beiden Partnern abgestellt werden und können ihre eigentlichen Aufgaben in ihren Unternehmen nicht mehr oder nur eingeschränkt wahrnehmen. Je kürzer die Planungsperiode ist, desto zeitintensiver wird der Planungsprozess. Im Extremfall wird das Koordinationsteam zu einer ständigen Einrichtung, etwa im Rang eines Stabes, der beiden Unternehmensleitungen zugleich unterstellt ist. Die Mitglieder würden dann aus ihren ursprünglichen Funktionen vollständig ausscheiden.

In der Theorie lässt sich das Problem dieser Trade-offs durch ein Optimierungsmodell lösen. Man legt für die Größen Flexibilität, Verlässlichkeit und Periodenlänge Anspruchsniveaus fest, die dann als Restriktionen in das Modell eingehen, und erklärt die Planungskosten zur Zielgröße, die zu mini-

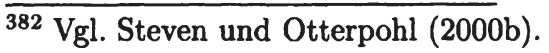


mieren ist. Dieses Vorgehen ist mit zwei Mängeln behaftet. Zum einen sagt das Modell nichts darüber, wie die Anspruchsniveaus zu bestimmen sind. Sie gehen vielmehr als exogene Größen in das Modell ein. $\mathrm{Zu}$ ihrer Festlegung wären weitere Optimierungsmodelle oder Heuristiken heranzuziehen. Zum anderen gibt es Schwierigkeiten bei der Operationalisierung aller Größen des Modells. Für die Periodenlänge ergibt sich zwar kein Problem, und auch die Planungskosten lassen sich bei allen Abgrenzungsproblemen im Einzelnen wohl noch messen. Für Flexibilität und Verlässlichkeit jedoch gibt es kein „natürliches“ Maß. Man muss Kennziffern wie Durchlaufzeiten oder Servicegrade heranziehen, die die gewünschte Größe unvollkommen abbilden.

Die dargestellten Gründe sind natürlich nicht hinreichend, um die Erarbeitung eines gemeinsamen Wirtschaftsplans grundsätzlich nicht empfehlenswert zu machen. Wir untersuchen hier den Fall, dass die Partner die Argumente in ihrer konkreten Situation für schwerwiegend genug halten, um auf die Einrichtung einer unternehmensübergreifenden Koordinationsinstanz zu verzichten. Transaktionskosten sollen soweit wie möglich eingespart werden, so dass die Partner auch auf sonstige bindende Absprachen, deren Einhaltung ja wieder kontrolliert werden müsste, verzichten. Statt dessen vereinbaren sie, sich auf einen grundlegenden Kreuzanpassungsprozess zu verständigen, d.h. jeder Partner wählt seinen Wirtschaftsplan für die kommende Periode auf der Basis sowohl des eigenen Plans der gegenwärtigen Periode als auch des Plans des Partners, soweit er sich in Produktion und Absatz manifestiert hat. Sie wissen, dass ein Nash-Gleichgewicht mit gewissen Stabilitätseigenschaften für die Partnerschaft existiert, und vertrauen darauf, dass sie sich durch den vereinbarten Prozess stets dem Gleichgewicht annähern. Ob sie es jemals erreichen, hängt von der Dynamik der Umwelt ab, doch ist die permanente Annäherung an das statische Optimum das Beste, was sich unter den gegebenen Bedingung erreichen lässt. Es spielt keine Rolle, ob die Entscheidungsträger spieltheoretisch geschult sind und mit dem Begriff des Nash-Gleichgewichts umzugehen wissen. Es kommt einzig darauf an, dass sie das Gleichgewicht als Win-Win-Situation erkennen und nutzen.

In Übereinstimmung mit dem Schema von Schäffer ${ }^{383}$ kann man davon sprechen, dass die Koordination in Selbstabstimmung erfolgt, auch wenn der Begriff in der Literatur nicht einheitlich verwendet wird und das Szenario mit dem Begriffsverständnis mancher Autoren von Selbstabstimmung nicht vereinbar ist. ${ }^{384}$ Die Produktion in Kooperation ist ein Sachziel, das die beiden Partner gemeinsam verfolgen. Die Gewinnerzielung durch Erfüllung des

${ }^{383}$ Vgl. wiederum Abbildung 4.1 auf S. 111.

${ }^{384}$ Vgl. Schäffer (1996) und die Diskussion in Abschnitt 4.3. 
Sachziels ist nur durch Zusammenarbeit zu erreichen. Es liegt also ein gemeinsamer Zweck auf Sach- wie auf Formalzielebene vor. Da die Konstellation hierarchiefrei gestaltet ist, ohne Weisungsbefugnisse des einen Partners dem anderen gegenüber, erfolgt die Koordination horizontal.

Im Folgenden wird das beschriebene Szenario formalisiert. Zunächst zeigen wir die Existenz und Optimalität des statischen Nash-Gleichgewichts. Dann wird ein adaptiver Kreuzanpassungsprozess definiert, der sich von der

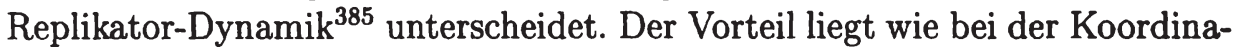
tion über Verrechnungspreise ${ }^{386}$ in den geringen Informationsanforderungen.

\subsubsection{Die Elemente des Modells}

Die Akteure im Modell zur Selbstabstimmung $\mathcal{S}$ sind zwei Partner $\mathcal{X}$ und $\mathcal{Y}$, die gemeinsam ein Produkt in der Menge $z^{s} \in \mathbb{R}_{+}$auf den Markt bringen. Sie sehen sich dort einer Marktnachfrage $z^{d} \in \mathbb{R}_{+}$gegenüber, die allein vom Preis $p \in \mathbb{R}_{+}$des Produktes abhängt. Für die Herstellung des Produktes sind die Beiträge $x \in \mathbb{R}_{+}$und $y \in \mathbb{R}_{+}$der Unternehmen erforderlich nach Maßgabe der Produktionsfunktion

$$
z: \mathbb{R}_{+}^{2} \rightarrow \mathbb{R}_{+},(x, y) \mapsto z .
$$

Beide Unternehmen maximieren nur ihre eigenen Gewinne; eine Kooperationsbereitschaft in dem Sinne, dass ein Unternehmen auf einen Teil seines Gewinns zugunsten der Kooperation zu verzichten bereit ist, wird also nicht angenommen.

Befassen wir uns zunächst mit der Marktnachfragefunktion. Häufig wird aus Gründen der Einfachheit auf eine explizite entscheidungslogische Fundierung verzichtet und eine lineare Nachfragefunktion angenommen. Für dieses Modell wird jedoch auf Basis eines Nutzenmaximierungskalküls eine konkrete aggregierte Nachfrage begründet. Im Ergebnis erhalten wir eine hyperbolische Funktion, die der Nachfrage der empfangenden Stelle im Verrechnungspreismodell $\mathcal{V}$ entspricht. Die Unterschied liegt darin, dass in $\mathcal{V}$ eine individuelle Faktornachfrage aus einer gegebenen Technologie hergeleitet wird, in $\mathcal{S}$ hingegen eine aggregierte Konsumnachfrage aus Nutzenmaximierungskalkülen.

Auf dem Markt für Produkt $z$ treten $n$ potenzielle Konsumenten als Nachfrager auf. Sie verwenden ihre jeweiligen Einkommen $c_{i} \in \mathbb{R}_{+}, i=1, \ldots, n$ auf Gut $z$, für das sie den Preis $p$ zahlen, sowie auf ein Güterbündel $\mathbf{w} \in \mathbb{R}_{+}^{m}$

385 Vgl. Abschnitt 5.1.4.

386 Vgl. Abschnitt 5.2.2. 
mit dem zugehörigen Preisvektor $\mathbf{q} \in \mathbb{R}_{+}^{m}$; die Einkommen $c_{i}$ werden im Vektor $\mathbf{c} \in \mathbb{R}_{+}^{n}$ zusammengefasst. Um das Problem so einfach wie möglich zu halten, ersetzen wir $\mathbf{w}_{i}$ durch den Mengenindex $w_{i} \in \mathbb{R}_{+}$und $\mathbf{q}$ durch den Preisindex $q \in \mathbb{R}_{+}$. Lemma 5.14 klärt die Voraussetzungen, unter denen eine solche Aggregation über Güter möglich ist. ${ }^{387}$

\section{Lemma 5.14}

Sei der Preisvektor $\mathbf{q}$ stets proportional zu einem Vektor $\mathbf{q}_{0}$ mit dem Proportionalitätsfaktor $q$. Wir wählen als Preisindex $q$ und als Mengenindex $w=\mathbf{q}_{0} \cdot \mathbf{w}^{388}$ Dann gilt für die Nachfragefunktionen $\mathbf{w}(p, \mathbf{q}, c)$ als Lösung des nicht aggregierten Problems

$$
v(p, \mathbf{q}, c)=\max _{z, \mathbf{w}} u(z, \mathbf{w}) \text { s.t. } p \cdot z+\mathbf{q} \cdot \mathbf{w}=c
$$

und die Nachfragefunktion $w(p, q, c)$ als Lösung des aggregierten Problems

$$
V(p, q, c)=\max _{z, w} U(z, w) \text { s.t. } p \cdot z+q \cdot w=c
$$

die Identität

$$
w(p, q, c)=\mathbf{q}_{0} \cdot \mathbf{w}(p, \mathbf{q}, c)
$$

Beweis. Unter Verwendung der Proportionalitätsbedingung schreiben wir den Nutzenmaximierungskalkül

$$
v(p, q, c)=\max _{z, \mathbf{w}} u(z, \mathbf{w}) \text { s.t. } q \cdot \mathbf{q}_{0} \cdot \mathbf{w}+p \cdot z=c .
$$

Eine Anwendung von Roys Identität ${ }^{389}$ zeigt mit Hilfe des Umhüllendentheorems ${ }^{390}$, dass sich die Definition des Mengenindex auf die Indexfunktion $w(q, p, c)$ und die Nachfragefunktionen $\mathbf{w}(p, \mathbf{q}, c)$ überträgt:

$$
w(p, q, c)=-\frac{\partial v(p, q, c) / \partial q}{\partial v(p, q, c) / \partial c}=\mathbf{q}_{0} \cdot \mathbf{w}(p, \mathbf{q}, c) .
$$

Wir kommen zum selben Ergebnis unabhängig davon, ob wir zuerst aggregieren und die Nachfrage für das gesamte Güterbündel berechnen oder

${ }^{387}$ Die im Lemma verwendete Form der Separierbarkeit wird als Hickssche Separierbarkeit bezeichnet. Vgl. Varian (1992), S. 148f.

${ }^{388}$ Die Indizierung der Variablen $\mathrm{w}, w, z$ und $c$ sowie der Funktionen $u, v, U$ und $V$ wird in der Formulierung dieses Lemmas einschließlich Beweis weggelassen.

${ }^{389} \mathrm{Vgl}$. Varian (1992), S. 106.

390 Vgl. Varian (1992), S. 502. 
zuerst die Nachfragen für die einzelnen Güter berechnen und diese aggregieren. Die Forderung nach der Proportionalität des Preisvektors q zu einem Basispreisvektor $\mathbf{q}_{0}$ bedeutet nichts anderes, als dass die relativen Preise der Güter w zueinander konstant bleiben. Daher ist es, wie Lemma 5.14 gezeigt hat, gerechtfertigt, über die Güter w zu aggregieren; die Indizes $q$ und $w$ sind geeignet, um das Güterbündel wie ein einziges Gut zu behandeln. Da allein das Gut $z$ im Mittelpunkt der Betrachtung steht und die übrigen Güter w lediglich dazu dienen, das Modell zu schließen, bedeutet die Annahme konstanter relativer Preise q keine Einschränkung.

\section{Satz 5.15}

Jeder Konsument $i=1, \ldots, n$ besitze Präferenzen, die er unabhängig von den anderen Konsumenten maximiert und die durch eine Cobb-DouglasNutzenfunktion der Form

$$
u_{i}\left(z_{i}, v_{i}\right)=z_{i}^{\gamma_{i}} \cdot w_{i}^{1-\gamma_{i}}, 0<\gamma_{i}<1
$$

repräsentiert werden. Sei außerdem $\sum_{i=1}^{n} \gamma_{i} \cdot c_{i}=1.391$

Dann lautet die aggregierte Nachfrage nach Gut $z$

$$
z^{d}(p)=\frac{1}{p}
$$

Beweis. Wir wählen als indirekte Nutzenfunktion ${ }^{392}$

$$
\begin{gathered}
v_{i}\left(p, q, c_{i}\right)=\max _{z_{i}, w_{i}} \gamma_{i} \cdot \ln z_{i}+\left(1-\gamma_{i}\right) \ln w_{i} \\
\text { s.t. } \quad p \cdot z_{i}+q \cdot w_{i}=c_{i} .
\end{gathered}
$$

Aus der zugehörigen Lagrange-Funktion erhalten wir die notwendigen Bedingungen:

$$
\begin{aligned}
\frac{\gamma_{i}}{z_{i}}-\lambda \cdot p & =0, \\
\frac{1-\gamma_{i}}{w_{i}}-\lambda \cdot q & =0 \\
-p \cdot z_{i}-q \cdot w_{i}+c_{i} & =0 .
\end{aligned}
$$

${ }^{391}$ Diese Normierung dient ausschließlich dazu, einen Systemparameter zu eliminieren, dessen Auswirkungen auf die Systemdynamik nicht veränderbar ist.

392 Die Darstellung der Präferenzstruktur durch eine direkte Nutzenfunktion ist lediglich bis auf monotone positive Transformationen bestimmbar. Der Einfachheit halber wird hier deswegen die logarithmische Form der Cobb-Douglas-Nutzenfunktion verwendet. 
Das Auflösen dieses Gleichungssystems ergibt die individuelle Nachfrage nach Gut $z$ :

$$
z_{i}^{d}\left(p, c_{i}\right)=\frac{\gamma_{i} \cdot c_{i}}{p}
$$

Wir prüfen die geränderte Hesse-Matrix auf ihr Vorzeichen:

$$
\operatorname{det}(\text { Hess })=\operatorname{det}\left(\begin{array}{ccc}
0 & -p & -q \\
-p & -\frac{\gamma_{i}}{z_{i}^{2}} & 0 \\
-q & 0 & -\frac{1-\gamma_{i}}{w_{i}^{i}}
\end{array}\right)=p^{2} \cdot \frac{1-\gamma_{i}}{w_{i}^{2}}+q^{2} \cdot \frac{\gamma_{i}}{z_{i}^{2}}>0
$$

Also maximiert Gleichung (5.43) den individuellen Nutzen von Konsument $i$.

Die aggregierte Konsumnachfrage nach Gut $z$ erhält man durch Addition der individuellen Nachfragen ${ }^{393}$ :

$$
z^{d}(p)=\sum_{i=1}^{n} z_{i}^{d}\left(p, c_{i}\right)=\frac{1}{p} \sum_{i=1}^{n} \gamma_{i} \cdot c_{i}=\frac{1}{p} .
$$

Wir werden später die Umkehrfunktion der Nachfragefunktion (5.42) verwenden, die Preis-Absatz-Funktion

$$
p\left(z^{d}\right)=\left(z^{d}(p)\right)^{-1}=\frac{1}{z^{d}} .
$$

Wir wenden uns nun der Angebotsseite des Modells zu. Um eine interessante Dynamik zu erhalten, unterstellen wir kein Konkurrenzgleichgewicht, das einen „Nullpunkt von Wettbewerb“ ${ }^{\text {“394 }}$ darstellt. Um andererseits das Modell nicht unnötig zu komplizieren, nehmen wir an, dass die Kooperation der Partner $\mathcal{X}$ und $\mathcal{Y}$ ein Monopol auf Gut $z$ innehat; es gebe keine weiteren Konkurrenten, auch keine solchen, die ähnliche, von den Konsumenten als Substitute betrachtete Produkte anbieten. Dennoch können die Partner nicht ohne Weiteres ein Kartell bilden und sich wie ein Monopolist verhalten. Solche Kartelle neigen zur Instabilität, weil sich den Partnern Spielräume eröffnen, auf Kosten des anderen zusätzliche Gewinne zu erzielen.

Die Partner $\mathcal{X}$ und $\mathcal{Y}$ verfügen über linear-homogene Technologien zur Herstellung von $x$ und $y$. Wir leiten daraus die jeweiligen Kostenfunktionen ab.

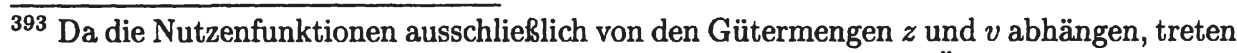
keine Interdependenzen in Form von Bandwagon-Effekten oder Ähnlichem auf.

${ }^{394}$ Schneider (1995a), S. 298. 
Satz 5.16

Seien die linear-homogenen Produktionsfunktionen der Unternehmen gegeben durch:

$$
\begin{aligned}
& \phi_{x}: \mathbb{R}_{+}^{k_{x}} \rightarrow \mathbb{R}_{+}, \quad \mathbf{r}_{x} \mapsto x, \\
& \phi_{y}: \mathbb{R}_{+}^{k_{y}} \rightarrow \mathbb{R}_{+}, \quad \mathbf{r}_{y} \mapsto y .
\end{aligned}
$$

$\mathbf{s}_{x}$ und $\mathbf{s}_{y}$ bezeichnen die Preise der Produktionsfaktoren $\mathbf{r}_{x}$ und $\mathbf{r}_{y}$. Die Minimalkostenkombinationen für $x=y=1$ heißen $\mathbf{r}_{x}^{*}$ bzw. $\mathbf{r}_{y}^{*}$. Dann lauten die Kostenfunktionen der Unternehmen

$$
\begin{aligned}
& c_{x}\left(\mathbf{s}_{x}, x\right)=\mathbf{s}_{x} \cdot \mathbf{r}_{x}^{*} \cdot x \\
& c_{y}\left(\mathbf{s}_{y}, y\right)=\mathbf{s}_{y} \cdot \mathbf{r}_{y}^{*} \cdot y .
\end{aligned}
$$

Beweis. Wir führen den Beweis für $c_{x}$. Aus der Definition von $\mathbf{r}_{x}^{*}$ folgt

$$
c_{x}\left(\mathbf{s}_{x}, 1\right)=\mathbf{s}_{x} \cdot \mathbf{r}_{x}^{*} .
$$

Wegen der Linearhomogenität gilt

$$
\phi_{x}\left(x \cdot \mathbf{r}_{x}^{*}\right)=x .
$$

Die Faktoreinsatzmenge $x \cdot \mathbf{r}_{x}^{*}$ genügt, um den Output $x$ zu produzieren. Es bleibt zu zeigen, dass $x \cdot \mathbf{r}_{x}^{*}$ kostenminimal ist. Sei angenommen, dass $\mathbf{r}_{x}^{\prime}$ geringere Kosten verursacht, also

$$
\mathbf{s}_{x} \cdot \mathbf{r}_{x}^{\prime}<x \cdot \mathbf{s}_{x} \cdot \mathbf{r}_{x}^{*}
$$

Dann ist aber auch

$$
\frac{1}{x} \cdot \mathbf{s}_{x} \cdot \mathbf{r}_{x}^{\prime}<\mathbf{s}_{x} \cdot \mathbf{r}_{x}^{*}
$$

$\frac{1}{x} \cdot \mathbf{r}_{x}^{\prime}$ genügt zur Produktion von $x=1$ und verursacht im Widerspruch zur Definition von $\mathbf{r}_{x}^{*}$ geringere Kosten. Also kann $\mathbf{r}_{x}^{\prime}$ nicht existieren; $x \cdot \mathbf{r}_{x}^{*}$ muss kostenminimal zur Produktion von $x$ sein.

Wir schreiben zur Vereinfachung

$$
\begin{aligned}
& c_{x}(x)=\alpha \cdot x \operatorname{mit} \alpha=\mathbf{s}_{x} \cdot \mathbf{r}_{x}^{*}, \\
& c_{y}(y)=\beta \cdot y \operatorname{mit} \beta=\mathbf{s}_{y} \cdot \mathbf{r}_{y}^{*} .
\end{aligned}
$$

Zur Aufstellung der Reaktionsfunktionen bleibt zu klären, wie der Gesamterlös $p \cdot z$ auf die Partner zu verteilen ist. Eine gleichsam naturgemäße 
Aufteilung gibt es nicht, die Partner müssen sich auf eine Regel verständigen. Solche Verteilungsregeln lassen sich grob in zwei Gruppen einteilen.

Zum einen kann man Regeln formulieren, die die Erlösanteile vorab und ohne Berücksichtigung der jeweiligen tatsächlich geleisteten Zielbeiträge festlegen. Die einfachste derartige Regel gibt jedem die Hälfte des Gesamterlöses, doch können die Anteile auch unterschiedlich groß sein. Darüber hinaus muss der Gesamterlös keineswegs ausgeschöpft werden. Statt dessen kann vereinbart werden, dass im Rahmen einer langfristigen Kooperation Erlöse thesauriert und in eine gemeinsame Infrastruktur investiert werden. Die Bestimmung der Anteile erfolgt in einem Verhandlungsprozess, in dessen Ergebnis sich die Verhandlungsmacht der Partner niederschlägt. Es liegt nahe, sich an der „Wichtigkeit" der Unternehmen für die Kooperation zu orientieren, wenn der Verhandlungsprozess nicht modelliert, sondern sein Ergebnis postuliert werden soll. Ein mögliches Maß für die Wichtigkeit und Verhandlungsmacht der Partner sind deren Zielbeiträge. Da die Vereinbarung aber vorab getroffen werden soll, muss an den vermuteten Zielbeiträgen angeknüpft werden. Das Problem dieser Regeln liegt in der Entkopplung von Leistung und Entlohnung. Die Kostenersparnis infolge einer Verringerung der Leistung kommt allein dem betreffenden Unternehmen zugute. Die Erlösminderung hingegen wird mittels der Verteilungsregel auf alle beteiligten Unternehmen umgelegt. Es ist damit zu rechnen, dass diesem Anreiz zur Leistungsverringerung alle beteiligten Unternehmen nachgeben mit der Folge, dass die Kooperation schließlich zusammenbrechen wird. ${ }^{395}$ Eine solche Verteilungsregel müsste also ergänzt werden durch die Festlegung von Mindestanspruchsniveaus, die man kontrollieren und deren Verfehlen man gegebenenfalls sanktionieren müsste.

Zum anderen kann man die tatsächlich geleisteten Beiträge der Unternehmen als Maßstab zur Berechnung der Erlösanteile verwenden. Damit stehen die Erlösanteile nicht im Vorhinein fest; der Anreiz zur Leistungsverringerung ist nicht mehr gegeben. Problematisch ist die Messung der tatsächlich geleisteten Beiträge. Die Mengen $x$ und $y$ sind wegen unterschiedlicher Maßeinheiten und damit mangelnder Vergleichbarkeit ungeeignet. Eine Umrechnung in Geldeinheiten benötigt (Schatten-)Preise für die mengenmäßigen Beiträge. Hierzu bieten sich die Grenzkosten $\alpha$ und $\beta$ an, die effiziente Mengen $x$ und $y$ sicherstellen. Dazu muss gewährleistet sein, dass die Grenzkosten vom jeweiligen anderen Partner nachgeprüft werden können. Anderenfalls muss als

395 Es liegt eine typische Gefangenen-Dilemma-Struktur vor. Vgl. Rasmusen (1994), S. 16. Die ursprüngliche Version des Gefangenen-Dilemmas beinhaltet zwei Beteiligte, eine Verallgemeinerung auf $n$ Personen ist aber ohne weiteres möglich. Vgl. hierzu Weimann (2001), S. 123ff. 
Second-best-Lösung eine möglichst gute Näherung in einem Verhandlungsprozess gefunden werden, mit der sich Planungssicherheit und Anreizverträglichkeit, wenn auch nicht sicher Effizienz erreichen lassen.

Wir nehmen an, dass die Grenzkosten dem jeweils anderen Partner nicht bekannt sind. Die Gewichte bei der Berechnung der Anteile - Ergebnis der Verhandlung - werden beide auf Eins normiert. Damit engen wir die Betrachtung nicht auf den Fall gleicher „Wichtigkeit" der Partner ein, sondern die Maßeinheiten der Mengen $x$ und $y$ werden so gewählt, dass die Gewichte jeweils den Wert eine Geldeinheit pro Mengeneinheit $x$ bzw. $y$ annehmen. Damit hängen die Grenzkosten, die sich später als die Dynamik bestimmenden Parameter herausstellen werden, vom Verhandlungsergebnis ab, weil hierdurch die Maßeinheiten von $x$ und $y$ festgelegt werden. Da der Verhandlungsprozess aber exogen ist, hat dies auf die weitere Modellanalyse keinen Einfluss. Wir schreiben die Anteilsfunktionen $\psi_{x}$ und $\psi_{y}$ daher

$$
\begin{aligned}
& \psi_{x}: \mathbb{R}_{+}^{2} \rightarrow[0,1], \quad(x, y) \mapsto \frac{x}{x+y}, \\
& \psi_{y}: \mathbb{R}_{+}^{2} \rightarrow[0,1], \quad(x, y) \mapsto \frac{y}{x+y} .
\end{aligned}
$$

\subsubsection{Statische Eigenschaften: Nash-Gleichgewicht}

Mit der Produktions- und Nachfragefunktion für $z$, den Kostenfunktionen der Beiträge $x$ und $y$ und der Verteilungsregel können wir die Gewinnmaximierungskalküle der Unternehmen formulieren und die Reaktionsfunktionen ableiten.

\section{Satz 5.17}

Die Kalküle der Unternehmen sind unter Verwendung der Gleichungen (5.41), (5.44), (5.45) und (5.46) gegeben durch

$$
\begin{aligned}
& \pi_{x}(y)=\max _{x} \psi_{x}(x, y) \cdot p\left(z^{d}\right) \cdot z(x, y)-c_{x}(x)=\frac{x}{x+y}-\alpha \cdot x \\
& \pi_{y}(x)=\max _{y} \psi_{y}(x, y) \cdot p\left(z^{d}\right) \cdot z(x, y)-c_{y}(y)=\frac{y}{x+y}-\beta \cdot y
\end{aligned}
$$


Dann heißen ihre Reaktionsfunktionen ${ }^{396}$

$$
\begin{aligned}
& x: \mathbb{R}_{+}^{2} \rightarrow \mathbb{R}_{+}, \quad(y, \alpha) \mapsto \sqrt{\frac{y}{\alpha}}-y, \\
& y: \mathbb{R}_{+}^{2} \rightarrow \mathbb{R}_{+}, \quad(x, \beta) \mapsto \sqrt{\frac{x}{\beta}}-x .
\end{aligned}
$$

Beweis. Wir führen den Beweis für $x$. Notwendige Bedingung für ein Gewinnmaximum ist

$$
\frac{y}{(x+y)^{2}}-\alpha=0
$$

Die hinreichende Bedingung

$$
-\frac{2 y}{(x+y)^{3}}<0
$$

ist erfüllt. Durch Umstellen der notwendigen Bedingung erhält man

$$
x= \pm \sqrt{\frac{y}{\alpha}}-y
$$

wobei nur die positive Wurzel dem Definitionsbereich entspricht und ökonomisch relevant ist.

Die Reaktionsfunktionen werden in Abbildung 5.17 dargestellt. Beide Kurven verlaufen durch den Ursprung, erreichen jeweils bezüglich ihrer Abszisse ein Maximum und schneiden dann wieder die jeweilige Achse. Daher gibt es neben dem Ursprung in jedem Fall, d.h. bei jeder Ausprägung der Parameter, einen weiteren Schnittpunkt. Wir werden im Folgenden sehen, dass dieser Schnittpunkt die Eigenschaften eines Nash-Gleichgewichts aufweist.

Die Kooperation der Partner $\mathcal{X}$ und $\mathcal{Y}$ lässt sich als Spiel auffassen und analysieren. ${ }^{397}$ Die Partner selbst werden in der Menge der Spieler zusammengefasst. Die von ihnen geleisteten Beiträge $x$ und $y$ stellen ihre Strategien dar, so dass der Vektor $(x, y)$ das Strategienprofil beschreibt. ${ }^{398}$ Die Auszahlungen sind die Gewinne, erfasst im Auszahlungsprofil $\left(\pi_{x}, \pi_{y}\right)$. Das Konzept

${ }^{396}$ In Puu (1997), S. 134ff., wird dieses Gleichungssystem zur Analyse eines Oligopols verwendet. Puu kommt jedoch teilweise zu anderen numerischen Ergebnissen.

397 Vgl. hierzu nochmals Abschnitt 5.1.

${ }^{398}$ Die Begriffe Zielbeitrag, Menge und Strategie können in diesem Modell synonym verwendet werden. 


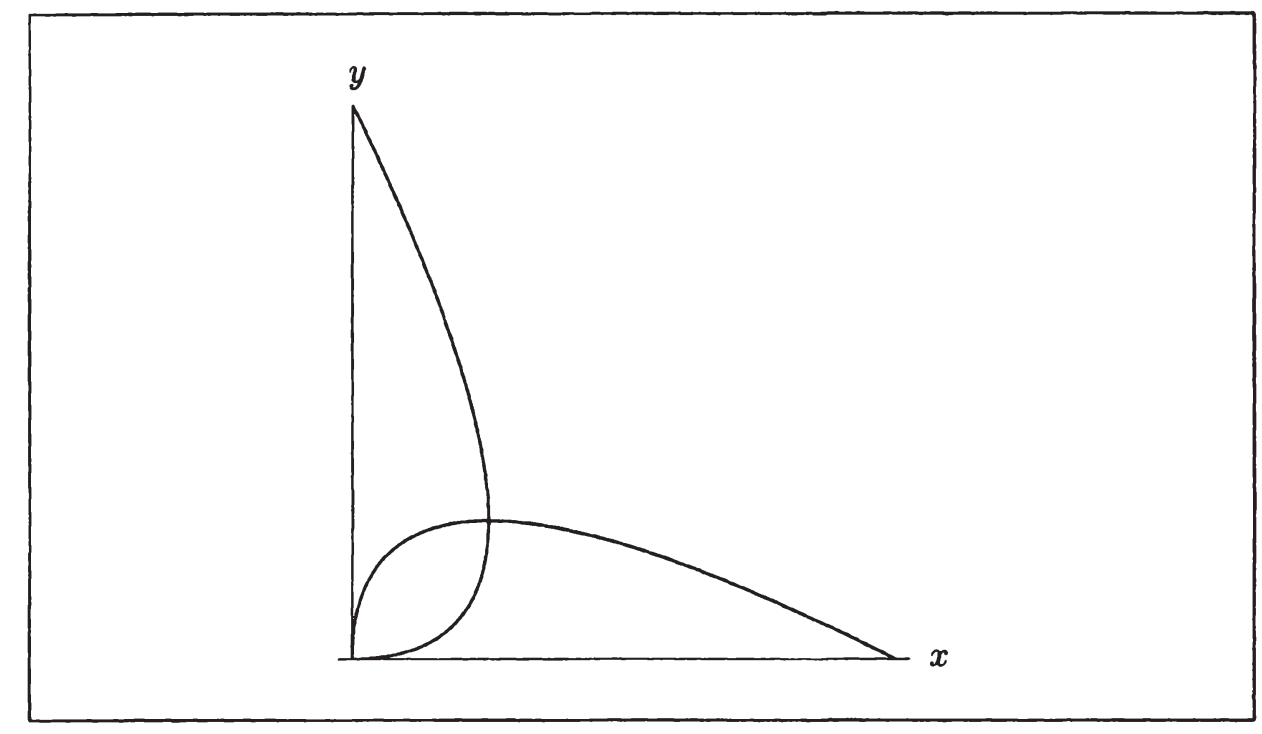

Abbildung 5.17: Reaktionsfunktionen des Modells $\mathcal{S}$ für $\alpha=\beta$

des Nash-Gleichgewichts ist anwendbar, allerdings mit leichten Modifikationen gegenüber Abschnitt 5.1.2.1. Dort verwendete Definition 5.2 die BesteAntwort-Korrespondenz auf der Basis endlich vieler mischbarer Strategien. Hier liegt der Fall etwas anders: Die Menge der reinen Strategien ist nicht endlich, da alle $(x, y) \geq 0$ zulässig sind und jede Menge als eigene Strategie anzusehen ist. Man könnte den Strategienraum künstlich einschränken, indem man nur eine endliche Zahl von Mengen zulässt; dies ist im vorliegenden Modell jedoch nicht erforderlich. Von gemischten Strategien als Wahrscheinlichkeitsverteilung über reine Strategien wird abgesehen; eine Einbeziehung verändert die Struktur des Modells nicht, da alle gemischten Strategien bereits eine reine Strategie darstellen.

Wegen der unendlichen Strategienmengen lässt sich das Spiel weder in strategischer Form noch in Bimatrix-Form sinnvoll darstellen. Statt dessen greift man auf Reaktionsfunktionen zurück, die auf dem Rationalitätsprinzip aufbauen und in denen das Lösungskonzept schon mitgedacht ist. Beide Spieler verhalten sich rational, indem sie unter Berücksichtigung der Strategie des anderen ihre Auszahlungen maximieren, wie es das Konzept des Nash-Gleichgewichts vorsieht. Dies erfolgt im Gewinnmaximierungskalkül. Die daraus abgeleiteten Reaktionsfunktionen geben die beste Antwort bei gegebener Strategie des anderen Spielers an. In Bezug auf die dargestellte Situation definieren wir: 


\section{Definition 5.4}

Ein Strategienprofil $\left(x^{*}, y^{*}\right)$ heißt Nash-Gleichgewicht des Modells $\mathcal{S}$, wenn gilt: $\left(x^{*}, y^{*}\right)$ ist Lösung des Gleichungssystems

$$
\begin{aligned}
& x=\sqrt{\frac{y}{\alpha}}-y, \\
& y=\sqrt{\frac{x}{\beta}}-x .
\end{aligned}
$$

Ein solches Strategienprofil genügt den Anforderungen des NashGleichgewichts: $x^{*}$ maximiert den Gewinn $\pi_{x}$ für ein gegebenes $y$, ist also beste Antwort auf $y^{*}$, und vice versa. Daher gibt es für keinen Spieler einen Grund, vom Profil $\left(x^{*}, y^{*}\right)$ abzuweichen, und es ist ein Gleichgewicht im Sinne eines Ruhezustands erreicht.

\section{Satz 5.18}

Das Nash-Gleichgewicht des Modells $\mathcal{S}$ ist gegeben durch

$$
x^{*}=\frac{\beta}{(\alpha+\beta)^{2}} \quad \text { und } \quad y^{*}=\frac{\alpha}{(\alpha+\beta)^{2}} .
$$

Die Partner $\mathcal{X}$ und $\mathcal{Y}$ erzielen dabei Gewinne in Höhe von

$$
\pi_{x}^{*}=\frac{\beta^{2}}{(\alpha+\beta)^{2}} \quad \text { und } \quad \pi_{y}^{*}=\frac{\alpha^{2}}{(\alpha+\beta)^{2}} .
$$

Beweis. Die nash-gleichgewichtigen Strategien, d.h. die Mengen $x^{*}$ und $y^{*}$ erhält man durch simultanes Lösen des nichtlinearen Gleichungssystems (5.48). Aus den Reaktionsfunktionen ergibt sich unmittelbar: ${ }^{399}$

$$
\sqrt{\frac{y}{\alpha}}=\sqrt{\frac{x}{\beta}} \Longleftrightarrow y=\frac{\alpha}{\beta} \cdot x \Longleftrightarrow x=\frac{\beta}{\alpha} \cdot y .
$$

Eingesetzt in die erste bzw. zweite Reaktionsfunktion ergibt dies

$$
\begin{aligned}
& x=\sqrt{\frac{\frac{\alpha}{\beta} \cdot x}{\alpha}}-\frac{\alpha}{\beta} \cdot x \Longleftrightarrow x=\frac{\beta}{(\alpha+\beta)^{2}}, \\
& y=\sqrt{\frac{\frac{\beta}{\alpha} \cdot y}{\beta}}-\frac{\beta}{\alpha} \cdot y \Longleftrightarrow y=\frac{\alpha}{(\alpha+\beta)^{2}} .
\end{aligned}
$$

${ }^{399}$ Durch die Einschränkung des Definitionsbereichs auf nicht-negative reelle Zahlen sind die Umformungen äquivalent. 
Als hinreichende Bedingung müssen die Gewinnfunktionen an der kritischen Stelle konkav sein. Wir sehen:

$$
-\frac{y}{(x+y)^{2}}<0 \text { und }-\frac{x}{(x+y)^{2}}<0
$$

an allen Stellen mit $x, y>0$, also auch an der kritischen Stelle.

Die Gewinne der Unternehmen erhält man durch Einsetzen der optimalen Strategien in die Gewinnfunktionen.

Beide Unternehmen befinden sich nicht nur im eindeutigen NashGleichgewicht, d.h. es macht keinen Sinn, von den Mengen $x^{*}$ und $y^{*}$ abzuweichen. Sondern es sind auch beide Gewinne $\pi_{x}^{*}$ und $\pi_{y}^{*}$ positiv, so dass ein Anreiz, die Kooperation zu verlassen, nur besteht, wenn woanders höhere Gewinne zu erwarten sind. Unter Berücksichtigung der Risiken, die eine solche Veränderung mit sich bringt, kann man davon ausgehen, dass die Kooperation weitgehend stabil ist.

\subsubsection{Dynamische Eigenschaften in kontinuierlicher Zeit: Stabilität}

Wir prüfen nun die Stabilität des Nash-Gleichgewichts. Hierzu formulieren wir einen Anpassungsprozess in kontinuierlicher Zeit, $t \in \mathbb{R}$. Aus dem Satz 3.21 von Poincaré und Bendixson in Kapitel 3.5.2 wissen wir, dass ein solcher Anpassungprozess für Modell $\mathcal{S}$ nicht chaotisch verlaufen kann.

Die Veränderungsraten $\dot{x}$ und $\dot{y}$, also die Ableitungen nach der Zeit, seien abhängig von der Differenz zwischen der tatsächlichen Strategie zum Zeitpunkt $t$ und der gemäß den Reaktionsfunktionen (5.47) nashgleichgewichtigen Strategie.

$$
\begin{aligned}
& h_{x}: \mathbb{R} \rightarrow \mathbb{R}, \quad \sqrt{\frac{y}{\alpha}}-y-x \mapsto \dot{x}, \\
& h_{y}: \mathbb{R} \rightarrow \mathbb{R}, \quad \sqrt{\frac{x}{\beta}}-x-y \mapsto \dot{y} .
\end{aligned}
$$

An die genaue Form der Abhängigkeit lassen sich unterschiedlich strenge Anforderungen stellen. ${ }^{400}$ Abbildung 5.18 veranschaulicht die verschiedenen Anforderungsstufen durch beispielhafte Kurvenverläufe. Man kann als $\overline{400}$ Vgl. Steven und Otterpohl (2000a). 


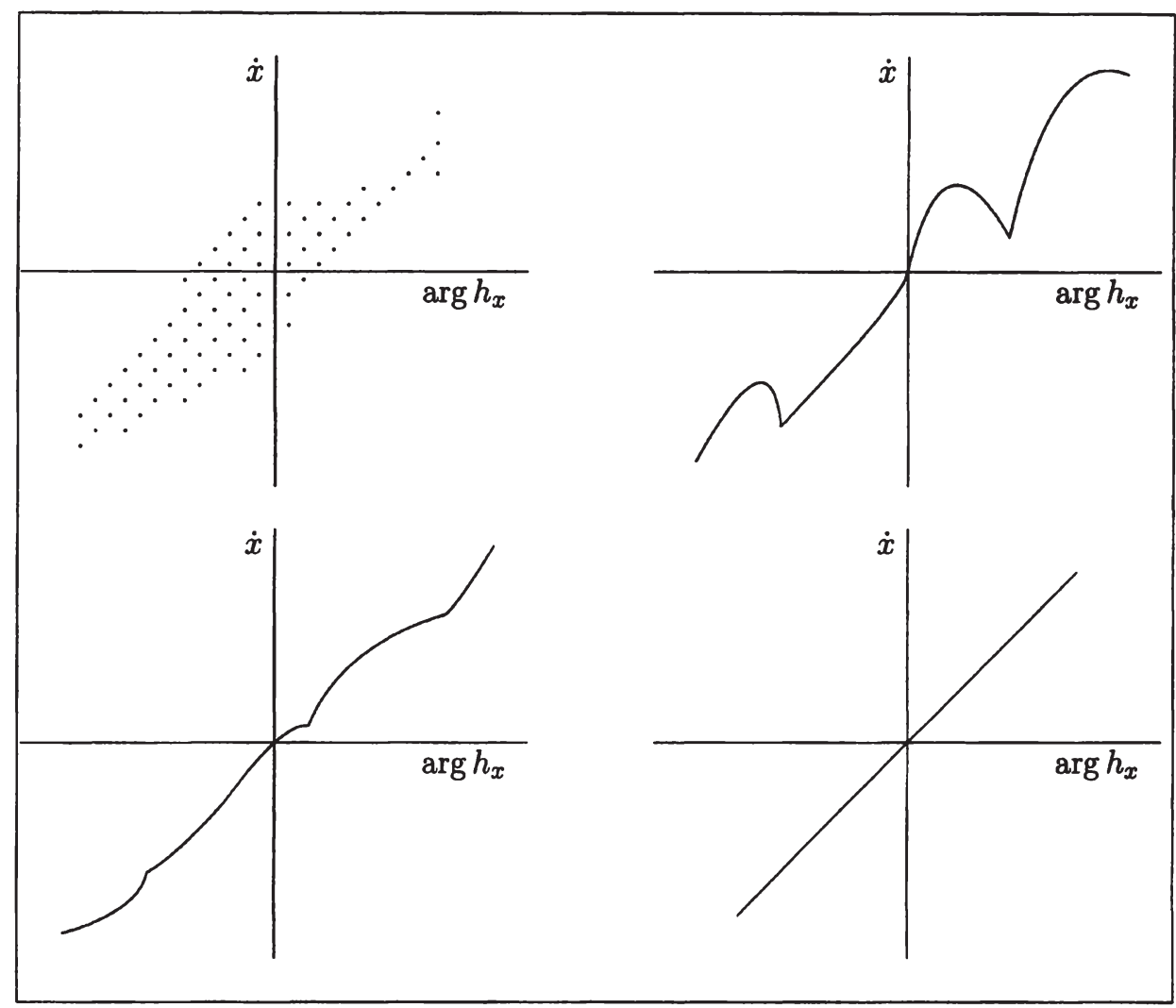

Abbildung 5.18: Veränderungsrate und Differenz

schwächste Anforderung lediglich eine positive statistische Korrelation verlangen. Hierzu muss der Anpassungsprozess eine stochastische Komponente aufweisen: ${ }^{401}$

$$
\operatorname{cov}\left(\dot{x}, \arg h_{x}\right)>0 .
$$

Bei deterministischem Anpassungprozess ist die Vorzeichengleichheit die schwächste Anforderung. Hierbei muss die Veränderungsrate $\dot{x}$ positiv sein, wenn $x<\sqrt{\frac{y}{\alpha}}-y$ und vice versa. Dadurch ist sichergestellt, dass sich die tatsächliche Strategie der nash-gleichgewichtigen Strategie stets annähert:

$$
\operatorname{sign} \dot{x}=\operatorname{sign} \arg h_{x} .
$$

Schärfer ist die strenge Monotonie. Die Veränderungsrate $\dot{x}$ muss umso größer sein, je größer die Differenz $\arg h_{x}$ ist:

$$
\arg _{1} h_{x}<\arg _{2} h_{x} \Longleftrightarrow \dot{x}_{1}<\dot{x}_{2}
$$

$\overline{401}$ Die Formeln werden im Folgenden nur für $x$ angegeben und sind für $y$ analog zu bilden. 
bzw. bei Differenzierbarkeit von $h_{x}$

$$
h_{x}^{\prime}>0 \text {. }
$$

Die stärkste Anforderung ist schließlich die Proportionalität zwischen Veränderungsrate und Differenz. Sie lässt sich am leichtesten mathematisch handhaben: ${ }^{402}$

$$
\dot{x}=\lambda \cdot \arg h_{x}, \quad \lambda \geq 0 .
$$

Die folgende Stabilitätsanalyse geht von strenger Monotonie aus. Es gelte, dass $\dot{x}$ und $\dot{y}$ umso größer werden, je größer die Abweichung zwischen tatsächlichen und nash-gleichgewichtigen Strategien ist. Außerdem seien die Anpassungsfunktionen differenzierbar, so dass wir von folgenden Eigenschaften ausgehen:

$$
h_{x}^{\prime}>0, \quad h_{y}^{\prime}>0, \quad h_{x}(0)=h_{y}(0)=0 .
$$

Satz 5.19

Seien die Anpassungsprozesse gegeben durch

$$
\dot{x}=h_{x}\left(\sqrt{\frac{y}{\alpha}}-y-x\right) \quad \text { und } \quad \dot{y}=h_{y}\left(\sqrt{\frac{x}{\beta}}-x-y\right),
$$

wobei $h_{x}$ und $h_{y}$ diffenzierbar und streng monoton steigend sind sowie durch den Ursprung verlaufen. Dann ist das Nash-Gleichgewicht

$$
x^{*}=\frac{\beta}{(\alpha+\beta)^{2}} \quad \text { und } \quad y^{*}=\frac{\alpha}{(\alpha+\beta)^{2}}
$$

lokal asymptotisch stabil.

Beweis. Das Nash-Gleichgewicht (5.52) ist dann lokal asymptotisch stabil, wenn die Eigenwerte der Jacobi-Matrix D zu System (5.51) im Gleichgewicht negative Realteile besitzen. Dies gilt bei einem zweidimensionalen System genau dann, wenn die Spur der Jacobi-Matrix negativ, ihre Determinante aber positiv ist. ${ }^{403}$ Es gilt:

$$
\mathbf{D}=\left(\begin{array}{ll}
h_{x}^{\prime} \cdot \frac{\partial \dot{x}}{\partial x} & h_{x}^{\prime} \cdot \frac{\partial \dot{x}}{\partial y} \\
h_{y}^{\prime} \cdot \frac{\partial \dot{y}}{\partial x} & h_{y}^{\prime} \cdot \frac{\partial y}{\partial y}
\end{array}\right)=\left(\begin{array}{cc}
-h_{x}^{\prime} & h_{x}^{\prime} \cdot \frac{1}{2} \sqrt{\frac{1}{\alpha \cdot y}}-1 \\
h_{y}^{\prime} \cdot \frac{1}{2} \sqrt{\frac{1}{\beta \cdot x}}-1 & -h_{y}^{\prime}
\end{array}\right)
$$

402 Vgl. Friedman (1998a), S. 24.

${ }^{403}$ Vgl. Felderer und Homburg (1994), S. 426. Es handelt sich um einen Spezialfall des Routh-Hurwitz-Kriteriums. Vgl. hierzu Chiang (1984), S. 546. 
Wir prüfen:

$$
\begin{gathered}
\operatorname{sp}(\mathbf{D})=-h_{x}^{\prime}-h_{y}^{\prime}<0 \\
\left.\operatorname{det}(\mathbf{D})\right|_{x^{*}, y^{*}}=h_{x}^{\prime} \cdot h_{y}^{\prime} \cdot\left(\frac{1}{2}+\frac{\alpha}{4 \beta}+\frac{\beta}{4 \alpha}\right)>0 .
\end{gathered}
$$

\subsubsection{Dynamische Eigenschaften in diskreter Zeit: eindimensionale Variante}

Die Situation stellt sich anders dar, wenn der Anpassungsprozess in diskreten Schritten abläuft. Im Unterschied zur Analyse in kontinuierlicher Zeit werden die Strategien der beiden Unternehmen nun als Funktionen der diskreten Zeit $t \in \mathbb{Z}$ verstanden.

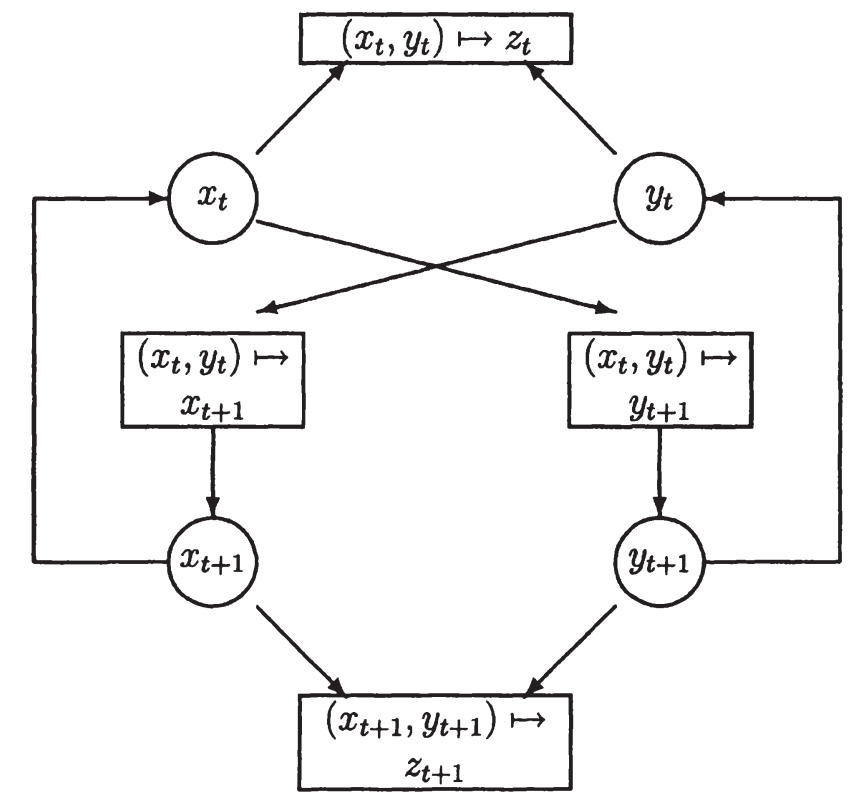

Abbildung 5.19: Scheinbar doppelter Regelkreis der eindimensionalen Variante

Die Nichtlinearität der Reaktionsfunktionen macht chaotisches Verhalten des Systems nun zumindest möglich. Die Unternehmen passen ihre Strategien adaptiv an das Koordinationsergebnis der gegenwärtigen Periode an. Die 
Wahl der adaptiven Anpassung wird wie im Modell $\mathcal{V}$ mit der Berücksichtigung von Lerneffekten und den geringen Informationsanforderungen begründet. ${ }^{404}$ Zunächst untersuchen wir den Spezialfall, in dem beide Adaptionsparameter den Wert Eins annehmen. Hier hat man es mit der unabhängigen Iteration zweier Variablen zu tun. Abbildung 5.19 zeigt den Regelkreis schematisch. Die Analogie zur kontinuierlichen Zeitmessung zeigt die Formulierung der Anpassungsprozesse mit Hilfe von Differenzenoperatoren:

$$
\begin{aligned}
\Delta x & =\sqrt{\frac{y_{t}}{\alpha}}-y_{t}-x_{t} \\
\Delta y & =\sqrt{\frac{x_{t}}{\beta}}-x_{t}-y_{t}
\end{aligned}
$$

Die übliche Form der Differenzengleichungen deckt die Unabhängigkeit der Iterationen auf:

$$
\begin{aligned}
& x_{t+1}=\sqrt{\frac{y_{t}}{\alpha}}-y_{t} \\
& y_{t+1}=\sqrt{\frac{x_{t}}{\beta}}-x_{t}
\end{aligned}
$$

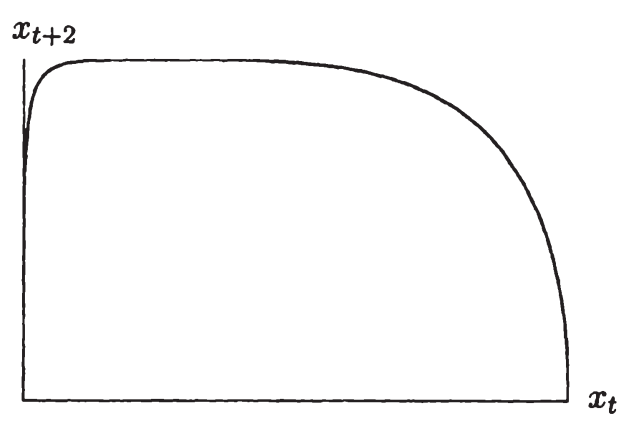

Abbildung 5.20: Reaktionsfunktion $x_{t} \mapsto x_{t+2}$ für $\alpha=\beta=1$

Durch Einsetzen der einen Gleichung in die andere erhält man eine Differenzengleichung mit nur noch einer Variablen. Der einzige Unterschied zu den gewohnten Iteratoren besteht lediglich darin, dass die Zeitverzögerung zwei Perioden beträgt, was aber für die Analyse ohne Bedeutung ist. Die

$\overline{404}$ Vgl. die Ausführungen in Abschnitt 5.2.2 auf S. 157f. 
Abbildung 5.20 zeigt die Gleichung

$$
x_{t+2}=\sqrt{\frac{1}{\alpha} \sqrt{\frac{x_{t}}{\beta}}-\frac{x_{t}}{\alpha}}-\sqrt{\frac{x_{t}}{\beta}}+x_{t}
$$

für $\alpha=\beta=1$. Die Ähnlichkeit mit der logistischen Parabel hat ein vergleichbares Szenario der Periodenverdopplungen ins Chaos zur Folge.

Der Verlauf des Anpassungprozesses hängt vom Verhältnis der Grenzkosten ab. Abbildung 5.21 zeigt Phasendiagramm und Bahnen für ein Verhältnis von $4: 1$ links und 6,25:1 rechts. Wegen der Zweidimensionalität des Systems sind die Phasendiagramme etwas anders zu lesen als etwa in den Abbildungen 5.7 und 5.14. Die beiden Kurven stellen die Reaktionsfunktionen dar; die Phasenpunkte $\left(x_{t}, y_{t}\right)$ liegen nicht auf den Kurven. Die Konvergenz zeigt sich im linken Teil der Abbildung 5.21 in Form einer Spirale in den Schnittpunkt der Kurven, das Nash-Gleichgewicht. Im rechten Teil scheinen die Phasenpunkte fast willkürlich im Phasenraum zu streuen. Der Übersichtlichkeit halber fehlen hier die Verbindungslinien der Phasenpunkte.

Je weiter die Grenzkosten der beiden Unternehmen auseinander liegen, desto länger dauert die transiente Phase bis zum Erreichen des Fixpunktes. Bei hinreichend großen Grenzkostenunterschieden - im vorliegenden Beispiel ab einem Verhältnis von ca. 5, $82: 1$ - beginnt mit der ersten Periodenverdopplung der Weg ins Chaos.

Das Bifurkationsdiagramm zeigt den auch aus den Modellen $\mathcal{B}$ und $\mathcal{V}$ bekannten Weg über Periodenverdopplungen. Wir untersuchen die Kaskade der Periodenverdopplungen genauer in den Bifurkationsdiagrammen in Abbildung 5.22. Die oberen beiden Diagramme zeigen die Endzustände der Strategievariablen $x$ und $y$, abgetragen gegen das Grenzkostenverhältnis $\alpha / \beta$. Wir sehen die üblichen Periodenverdopplungen bis zum Eintritt in chaotische Bänder, die von periodischen Fenstern unterbrochen werden. Übersteigt das Grenzkostenverhältnis den Wert 6, $25: 1$, so bricht das System zusammen. ${ }^{405}$

Das untere Diagramm weist eine Besonderheit auf. Hier ist gegen das Grenzkostenverhältnis $\alpha / \beta$ die Summe der Strategievariablen $x+y$ abgetragen. Diese Summe hat ökonomisch natürlich keine Bedeutung, sie dient hier nur der Aufdeckung der dynamischen Struktur des Systems. Die Besonderheit besteht darin, dass der Zyklus der Periode zwei fehlt. Der Grund

${ }_{405}$ Die mathematische Ursache des Zusammenbruchs besteht darin, dass die Radikanden in den Reaktionsfunktionen negativ und die Werte der Strategievariablen dadurch komplex werden. Ökonomisch sind komplexe Werte natürlich nicht mehr interpretierbar, d.h. das Modell ist in diesem Parameterbereich ökonomisch nicht mehr relevant. 

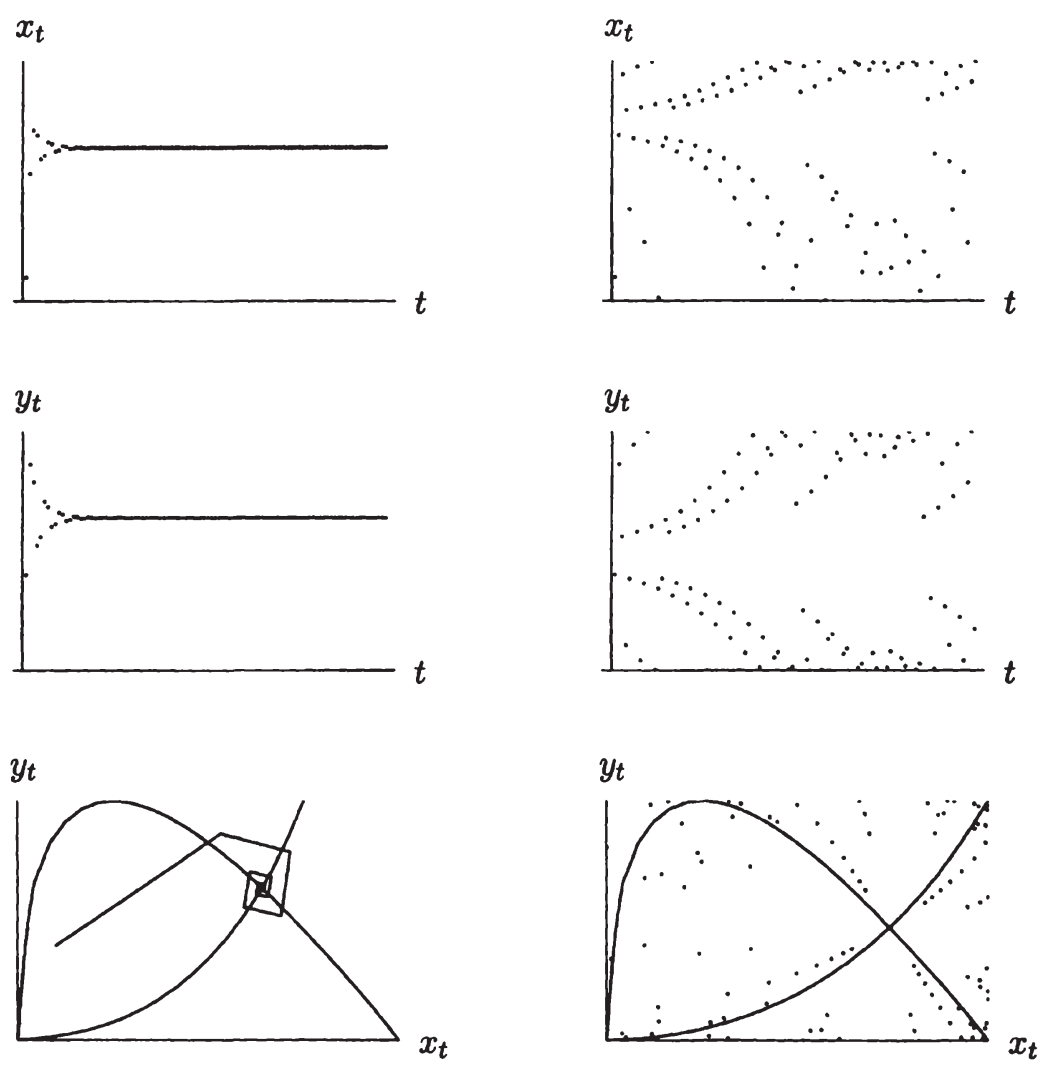

Abbildung 5.21: Phasendiagramme und Bahnen für $\alpha: \beta=1: 4$ (links) und $\alpha: \beta=1: 6,25$ (rechts)

liegt darin, dass die Werte $x_{t+1}$ bzw. $y_{t+1}$ ausschließlich von $y_{t}$ bzw. $x_{t}$ abhängen und sich das System in eine Funktion $x_{t} \mapsto x_{t+2}$ fassen lässt, wie in Gleichung (5.54) und Abbildung 5.20 bereits gezeigt wurde. ${ }^{406}$ Dadurch verdoppeln sich alle Periodenlängen, die Variablen $x_{t}$ und $y_{t}$ durchlaufen jeden Wert aber zweimal. Abbildung 5.23 zeigt beispielhaft Zyklen der Periode vier bzw. acht für die Variable $x_{t}$. Im Bifurkationsdiagramm werden für die entsprechenden Parameterwerte nur zwei Endzustände angezeigt. Da sich die Reihenfolgen, in denen die Zyklen durchlaufen werden, für $x_{t}$ und $y_{t}$ unterscheiden, unterscheiden sich alle Summen $x_{t}+y_{t}$ des gesamten Zyklus. So ist das Muster des vierperioden Zyklus dadurch gekennzeichnet, dass beide

$\overline{406}$ Äquivalent kann man natürlich auch eine Funktion $y_{t} \mapsto y_{t+2}$ bilden. 

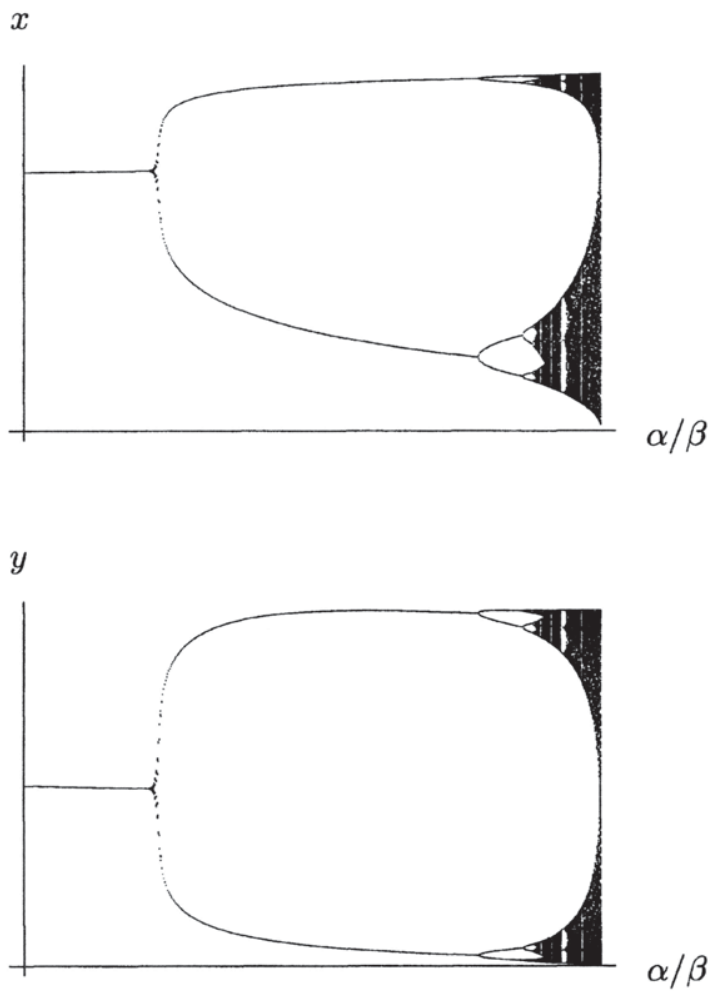

$x+y$

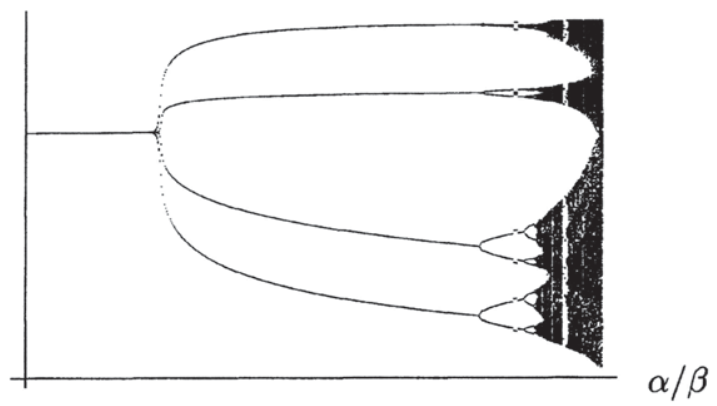

Abbildung 5.22: Bifurkationsdiagramme des Modells $\mathcal{S}$, eindimensionale Variante 

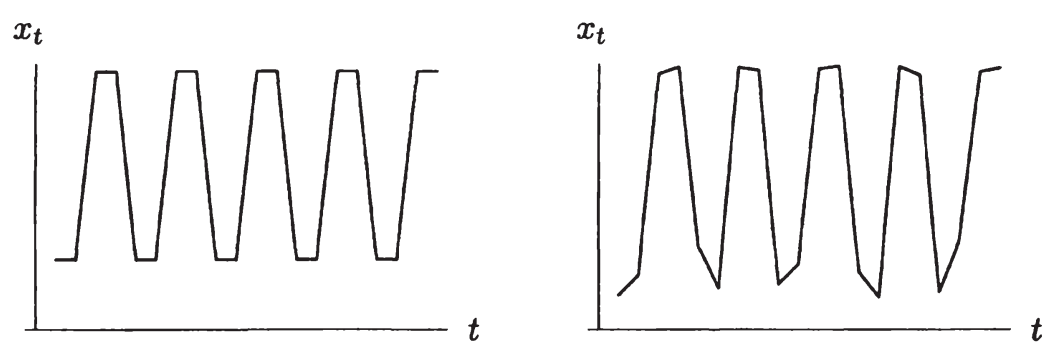

Abbildung 5.23: Bahnen für Zyklen der Periode vier und acht

Variablen zunächst zweimal den hohen Wert und dann zweimal den tiefen Wert annehmen. Allerdings ist dieses Muster für die beiden Variablen um eine Periode verschoben, so dass der hohe Wert für $x_{t}$ einmal auf den tiefen und einmal auf den hohen Wert für $y_{t}$ trifft, ebenso der tiefe Wert für $x_{t}$, so dass sich insgesamt vier Summen $x_{t}+y_{t}$ ergeben.

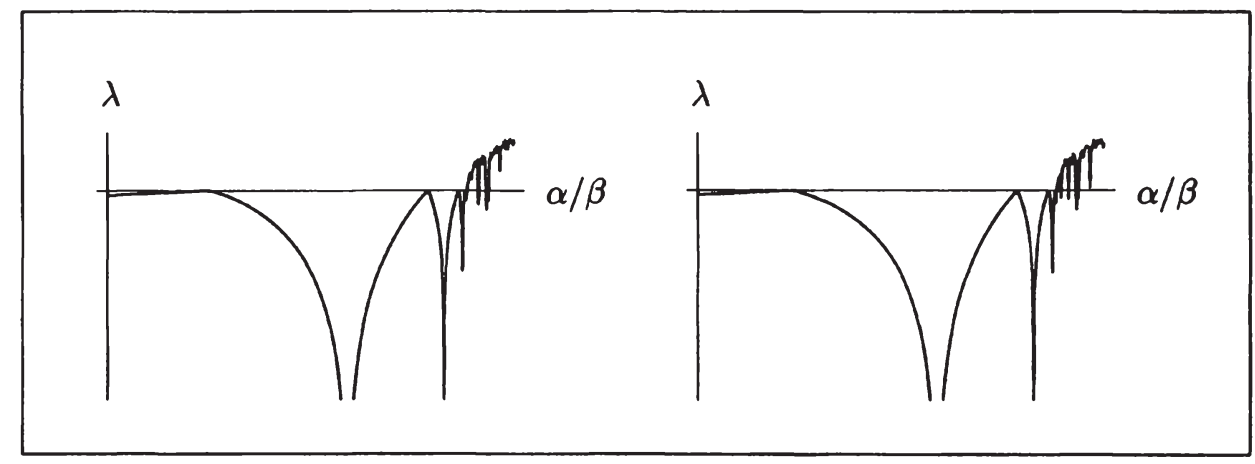

Abbildung 5.24: Lyapunov-Exponenten des Modells $\mathcal{S}$, eindimensionale Variante

Die Betrachtung der Lyapunov-Exponenten zeigt ein eher unerwartetes Bild. Nach den Ausführungen in Abschnitt 3.5.5 sollte bei einem zweidimensionalen System stets mindestens ein Exponent negativ sein, wie man in der Abbildung 3.15 für das Musterbeispiel des Hénon-Attraktors sehen konnte. Hier zeigt sich hingegen in Abbildung 5.24 ein beinahe identisches Sensitivitätsverhalten in $x$ - und $y$-Richtung. Wir haben es jedoch, wie bereits mehrfach betont, nicht mit einem echten zweidimensionalen System zu tun. Eine Konsequenz daraus ist, dass wir auch keinen seltsamen Attraktor finden, sondern der gesamte Phasenraum dicht gefüllt wird. Abbildung 5.25 stellt den Phasenraum nach 30.000 Iterationen dar. Das Auftreten sensitiver Abhängigkeit findet gleichmäßig in beide Achsenrichtungen statt. 


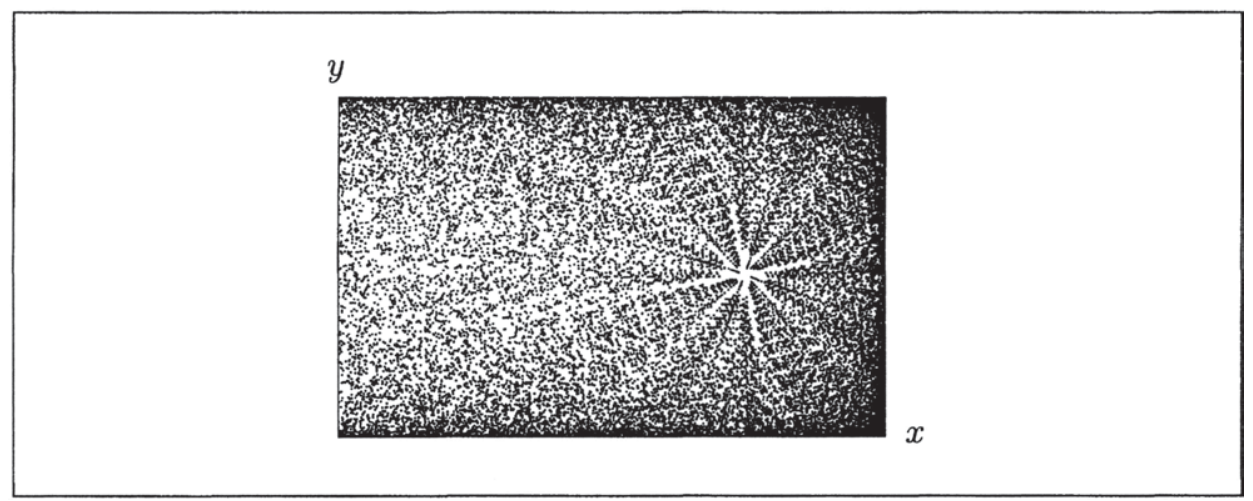

Abbildung 5.25: Phasendiagramm bei $\alpha / \beta=6,25$ nach 30.000 Iterationen

\section{Hypothese $\mathbf{5 . 2 0}$}

Das System (5.53) tritt für $\alpha: \beta=5,82: 1$ in ein Periodenverdopplungsszenario ein und wird für $\alpha: \beta=6,2: 1$ sensitiv in $x$ - und $y$-Richtung bei beschränktem Zustandsraum. Damit ist es numerisch chaotisch im Sinne von Definition 3.14 .

\subsubsection{Dynamische Eigenschaften in diskreter Zeit: zweidimensionale Variante}

In dem durch das Gleichungssystem (5.53) beschriebenen Modell gibt es praktisch keine Möglichkeit für die Unternehmensführung, aktive Maßnahmen zur Vermeidung langer transienter Phasen bzw. chaotischer Bereiche zu ergreifen. Der das Systemverhalten bestimmende Parameter ist das Verhältnis der Grenzkosten $\alpha / \beta$, das zum einen von der Technologie und zum anderen vom Ergebnis der Verhandlungen über die Verteilungsregel beeinflusst wird. ${ }^{407}$ Veränderungen können sich durch Innovationen, d.h. durch neue Produktionsanlagen oder -methoden ergeben. Doch ungeachtet der Tatsache, dass sich ein Unternehmen selbstverständlich ständig um Verbesserungen im Produktionsbereich bemühen muss, um wettbewerbsfähig zu bleiben, lässt sich eine Innovation nicht so planen, dass ihre Wirkung zur Vermeidung chaotischer Bereiche des Grenzkostenverhältnisses eingesetzt werden könnte. Das Grenzkostenverhältnis ließe sich auch durch den Verzicht auf individuelle Gewinnmaximierung annähern. Wenn schon der Partner mit den hohen Grenz-

${ }_{407}$ Man vergegenwärtige sich, dass die Gewichte der Anteile in den Verteilungsfunktionen $\psi_{x}$ und $\psi_{y}$ normiert sind. Das Verhandlungsergebnis wird durch die Wahl der Maßeinheiten der Produktmengen ausgedrückt und nimmt so Einfluss auf die Höhe der Grenzkosten. 
kosten diese nicht zu senken im Stande ist, so könnte doch der andere Partner seine niedrigen Grenzkosten erhöhen, also Ressourcen verschwenden. Dieser Ressourcenverschwendung müsste man nun die Kosten der Sensitivität gegenüber stellen, sofern letztere sinnvoll messbar sind. Und schließlich ist eine Nachverhandlung und anschließende Veränderung der Verteilungsfunktionen denkbar. Das Verhandlungsergebnis spiegelt jedoch die Machtverhältnisse wider, und einer Verschiebung der Verteilungsgewichte stimmt der Partner, zu dessen Ungunsten die Nachverhandlung ausgeht, nur dann zu, wenn er durch die Stabilisierung der Systemdynamik kompensiert wird.

Wir gehen einen anderen Weg, indem wir direkt an der Gestalt der Reaktionsfunktionen (5.53) ansetzen. Wir geben die Annahme auf, dass die Anpassungsgeschwindigkeiten gleich eins sind. Das Modell erhält dadurch einen echten zweidimensionalen Charakter, wie das Gleichungssystem (5.55) zeigt:

$$
\begin{aligned}
& x_{t+1}=x_{t}+\theta_{x}\left(\sqrt{\frac{y_{t}}{\alpha}}-y_{t}-x_{t}\right) \\
& y_{t+1}=y_{t}+\theta_{y}\left(\sqrt{\frac{x_{t}}{\alpha}}-x_{t}-y_{t}\right) .
\end{aligned}
$$

Das Gleichungssystem (5.53) ergibt sich durch Setzen von $\theta_{x}=\theta_{y}=1$. Um die numerische Analyse nicht unnötig zu verlängern, wird im Folgenden $\theta_{x}=\theta_{y}=\theta$ unterstellt. Der Regelkreis ist in Abbildung 5.26 dargestellt. Er unterscheidet sich vom Regelkreis in Abbildung 5.19 dadurch, dass $x_{t+1}$ auch von $x_{t}$ und $y_{t+1}$ auch von $y_{t}$ bestimmt wird.

Die zweidimensionale Form des Modells $\mathcal{S}$ enthält nun, neben den Systemvariablen $x_{t}$ und $y_{t}$, die Parameter $\alpha / \beta$ und $\theta$, also das Verhältnis der Grenzkosten und die Adaptionsgeschwindigkeit. Die numerische Analyse zeigt, dass für hinreichend hohe Grenzkostenunterschiede und Adaptionsparameter seltsame Attraktoren mit gebrochenem Dimensionsmaß auftreten. In Abbildung 5.27 sind von oben nach unten Attraktoren bei steigendem $\theta$ und $\alpha / \beta=6,25$ dargestellt. In den ersten fünf Phasendiagrammen scheint sich der Fixpunktattraktor auszudehnen, als würde er explodieren. Es liegt jedoch keine Bifurkation vor, der Attraktor besteht in allen Fällen nur aus dem Fixpunkt. Die Diagramme werden erstellt, indem man von einem beliebigen Punkt im Bassin des Attraktors ausgeht. Dann werden einige Iterationen zur Überwindung der transienten Phase durchgeführt und die darauf folgenden Iterationen, die hinreichend nah beim Attraktor liegen, schließlich in das Diagramm eingezeichnet. Im ersten Diagramm wird auf diese Weise in der Tat nur der Fixpunkt erfasst; die transiente Phase wird jedoch mit steigendem $\theta$ extrem 


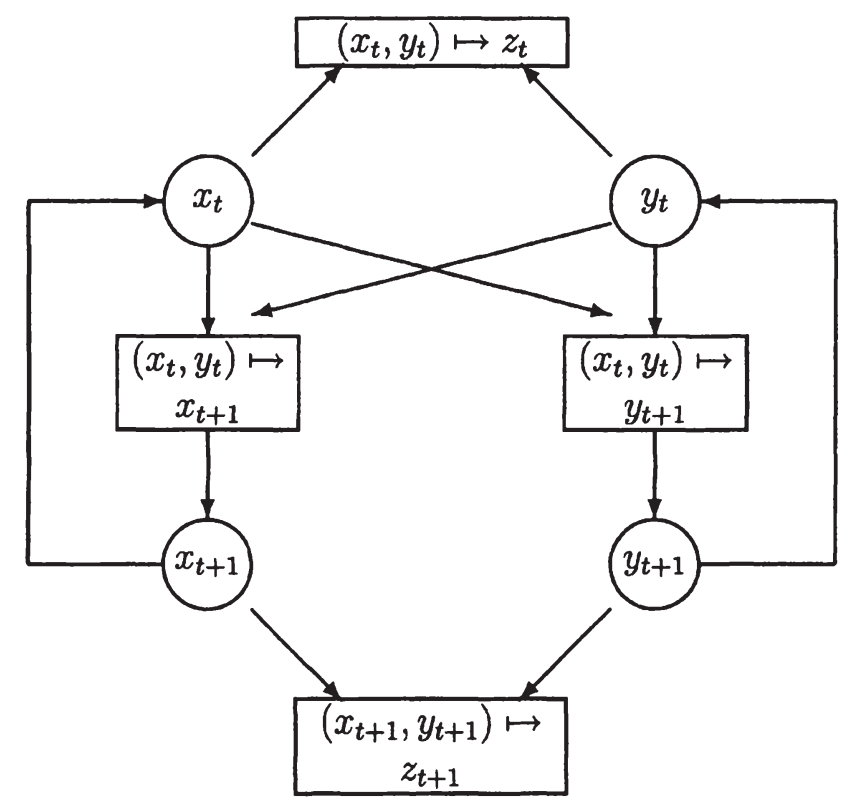

Abbildung 5.26: Echt doppelter Regelkreis der zweidimensionalen Variante

lang, so dass immer mehr Punkte in einiger Entfernung vom Attraktor in das Diagramm eingehen.

Die lange transiente Phase ist jedoch bereits Anzeichen für eine bevorstehende Änderung des Systemverhaltens. Aus dem Fixpunktattraktor entsteht ein Attraktor in Form einer geschlossenen Bahn. Man bezeichnet dies als Hopf-Bifurkation, deren Vorliegen sich auch analytisch nachweisen lässt. Satz 3.26 auf Seite 93 gibt die Kriterien an, die sich hier nachprüfen lassen.

Das System 5.55 besitzt bei Grenzkosten von $\alpha=6,25$ bzw. $\beta=1 \mathrm{im}$ Nash-Gleichgewicht die Jacobi-Matrix

$$
\mathbf{D}=\left(\begin{array}{cc}
1-\theta & -0,42 \cdot \theta \\
2,625 \cdot \theta & 1-\theta
\end{array}\right)
$$

mit den komplex-konjugierten Eigenwerten

$$
\lambda=1-\theta \pm 1,05 \cdot \theta \cdot i \text {. }
$$

Es gilt:

$$
|\lambda|=\sqrt{(1-\theta)^{2}+(1,05 \cdot \theta)^{2}}=1 \Longleftrightarrow \theta^{*}=\frac{800}{841} \approx 0,951 .
$$


Abbildung 5.27 bestätigt, dass der Übergang vom Fixpunkt zu einer geschlossenen Kurve zwischen $\theta=0,95$ und $\theta=0,96$ liegt. Berechnet man die ersten vier Potenzen von $\lambda$ an der Stelle $\theta^{*}=\frac{800}{841}$, erhält man sämtlich komplexe Werte, die auf dem Einheitskreis liegen, aber nicht gleichzeitig auf der reellen Achse, also ungleich \pm 1 sind. Und schließlich gilt:

$$
\left.\frac{d|\lambda|}{d \theta}\right|_{\theta^{*}=800 / 841}=\left.\frac{-1+1,05^{2} \cdot \theta}{\sqrt{(1-\theta)^{2}+(1,05 \cdot \theta)^{2}}}\right|_{\theta=800 / 841}=1>0
$$

Der Weg ins Chaos führt hier also nicht wie in den eindimensionalen Systemen über Periodenverdopplungen, sondern über eine Hopf-Bifurkation in eine geschlossene Kurve, die nach und nach gleichsam zerfasert. $\mathrm{Ab} \theta=0,951$ wird der größte Lyapunov-Exponent positiv, d.h. das System wird sensitiv. Erreicht $\theta$ den Wert 0,98, erhalten wir Lyapunov-Dimensionen zwischen eins und zwei, also einen seltsamen Attraktor mit fraktaler Struktur. Erreicht $\theta$ einen Wert sehr nahe bei eins, nähert sich das Systemverhalten dem Spezialfall aus Gleichung (5.53). Der Attraktor zerfällt zunächst in vier Teile, die bei $\theta=1$ zusammenwachsen und den gesamten Phasenraum ausfüllen.

Die Werte der Lyapunov-Dimensionen für steigendes $\theta$ sind in Abbildung 5.28 dargestellt. Der gebrochene Wert der Lyapunov-Dimension ist ökonomisch insoweit von Interesse, als er mit einem positiven LyapunovExponenten einher geht. Wenn man den Attraktor eines Systems wie Gleichung (5.55) im Phasenraum visualisiert und die Anschauung eine fraktale Struktur vermuten lässt, sind weiter gehende Tests auf Sensitivität dringend geboten.

Das System weist also auch in der zweidimensionalen Form sensitive Abhängigkeit von den Anfangsbedingungen auf mit den aus den Modellen $\mathcal{B}$ und $\mathcal{V}$ bekannten Folgen für die Prognostizierbarkeit und die Planbarkeit des Systems $\mathcal{S}$. In den Abbildungen 5.29 und 5.30 sieht man, dass es sich hier nicht um die bereits bekannten Periodenverdopplungen handelt, sondern der Weg ins Chaos hier über eine Hopf-Bifurkation verläuft.

Anhand der zweidimensionalen Version des Modells $\mathcal{S}$ lässt sich studieren, wie das Verhältnis mehrerer systembestimmender Parameter das dynamische Systemverhalten beeinflusst. Die Analyse zeigt somit, wie überaus wichtig es ist, sich aller Parameter bewusst zu werden. Die eindimensionale Modellvariante enthält nur das Grenzkostenverhältnis als Parameter. Die Adaptionsgeschwindigkeit ist hier auf Eins fixiert und damit aus dem Auge des Betrachters verschwunden. 

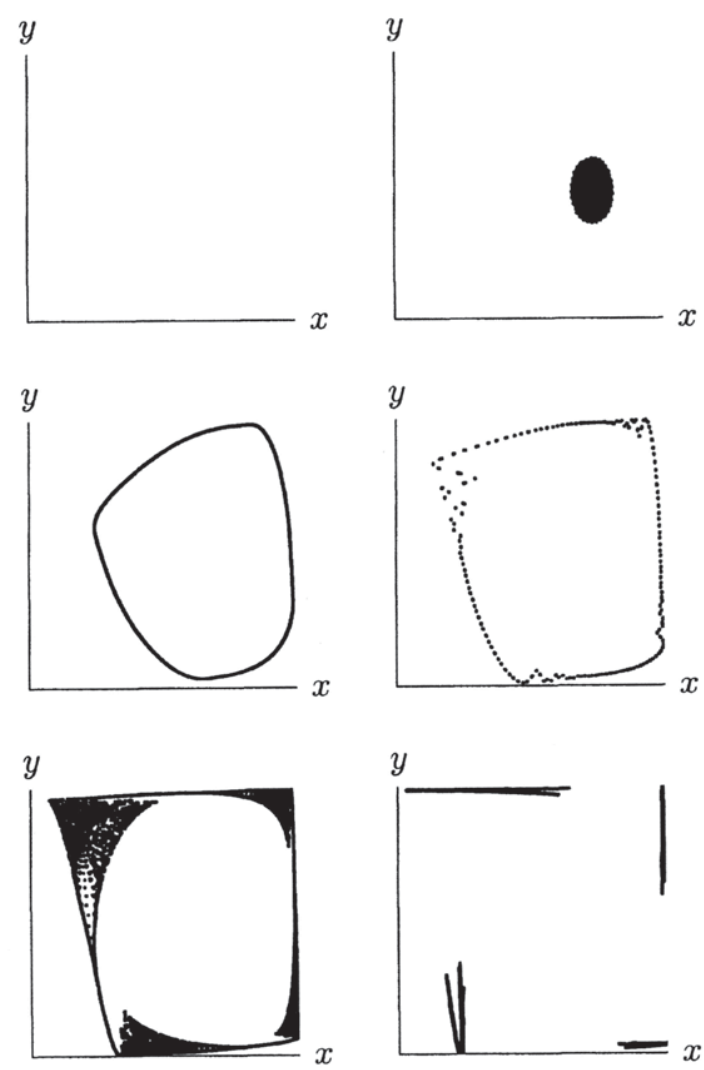

Abbildung 5.27: Attraktoren im $x$-y-Phasenraum für $\theta=0,94$ (oben links) bis $\theta=0,99$ (unten rechts)

Erst im zweidimensionalen Modell wird die Wechselwirkung verschiedener Parameter deutlich. Die Grenzkosten an sich sind nur um den Preis des Abrückens von der Gewinnmaximierung oder durch eine Nachverhandlung über die Verteilungsgewichte gezielt beeinflussbar. Endet die Analyse an dieser Stelle, muss man konstatieren, dass die Unternehmensführung keine zwingende Möglichkeit hat, das System aus einer chaotischen Region hinaus zu steuern. Der Einfluss der Grenzkosten kann aber durch den Adaptionsparameter konterkariert werden. Auch ein Grenzkostenverhältnis von mehr als 6,25:1, bei dem das eindimensionale Modell zusammenbricht, kann bei entsprechend niedriger Adaptionsgeschwindigkeit stabil sein. Interessant ist, dass die Aussage des Modells $\mathcal{V}$, die Adaptionsgeschwindigkeit nicht zu hoch 


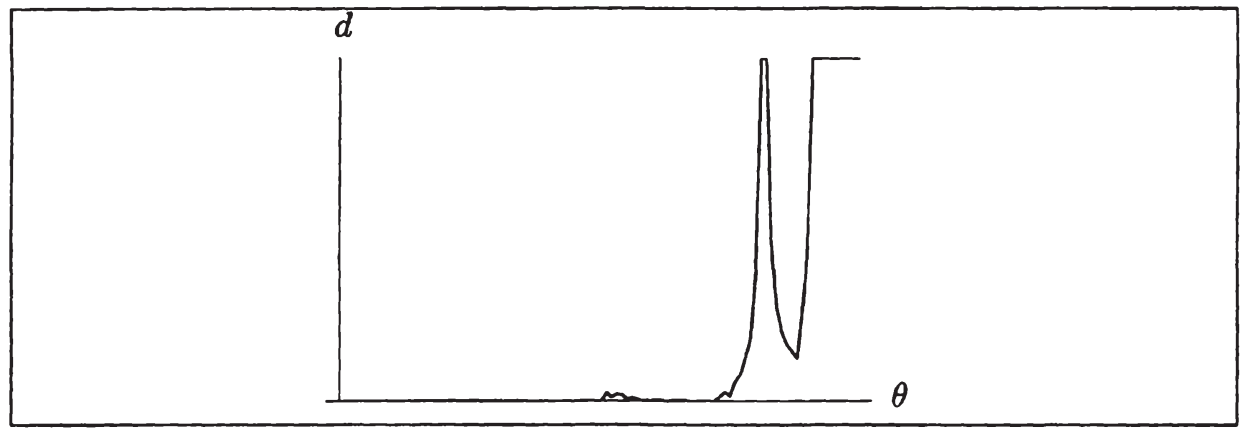

Abbildung 5.28: Lyapunov-Dimensionen des Modells $\mathcal{S}$, zweidimensionale Variante

zu wählen, auch in der völlig anderen Struktur des Modells $\mathcal{S}$ Gültigkeit besitzt. Der Grund liegt darin, dass eine „Politik der kleinen Schritte" hin zum Gleichgewicht ein Überschießen des Systemzustands verhindert.

Im besprochenen Modell haben die Partner auf jegliche zentrale Koordinationsinstanz und Absprache verzichtet. Statt dessen haben sie auf das Wirken des Kreuzanpassungsprozesses vertraut, der sie zum Nash-Gleichgewicht führen sollte. Wir haben jedoch gesehen, dass der Kreuzanpassungsprozess anfällig für Chaos ist und nur bei bestimmten Parameterkonstellationen zum gewünschten Ergebnis führt. Dies spricht trotz der oben angeführten Bedenken dafür, den Koordinationsmechanismus aufzugeben oder zumindest durch Elemente einer zentralen Planung zu ergänzen. In zentraler Planung werden $x$ und $y$ simultan und mit Blick auf den Gesamtgewinn festgelegt. Es ist daher nicht möglich, dass sich $x$ und $y$,aneinander vorbei“ entwickeln und einen seltsamen Attraktor formen. $\mathrm{Ob}$ damit auch die Entwicklung für die Partnerschaft insgesamt stabil verläuft, ließe sich nur in einem weiteren Modell klären. Von fallweisen Absprachen, wenn Fehlentwicklungen erkannt werden, bis zur Einrichtung eines ständigen, beiden Partnern gemeinsam unterstellten Koordinationsstabes gibt es eine ganze Reihe von Möglichkeiten. Wir verlassen damit den Bereich der Selbstabstimmung, das Modell $\mathcal{S}$ ist hierfür nicht geeignet. ${ }^{408}$ In jedem Fall wird aber der in Modell $\mathcal{S}$ Chaos verursachende Mechanismus durch eine zentrale Koordination ausgeschaltet. Was gegeneinander abzuwägen bleibt, und hier liegen Möglichkeiten für weitere Ansätze der Forschung, ist ein Vergleich der Kosten, die durch chaotische

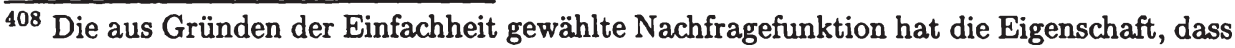
ein Monopolist bei jeder Preis-Mengen-Kombination denselben Erlös erzielt. Bei Gewinnmaximierung erhält man dadurch eine ökonomisch sinnlose Randlösung. Ohne zentrale Koordination tritt dieses Problem jedoch nicht auf. 

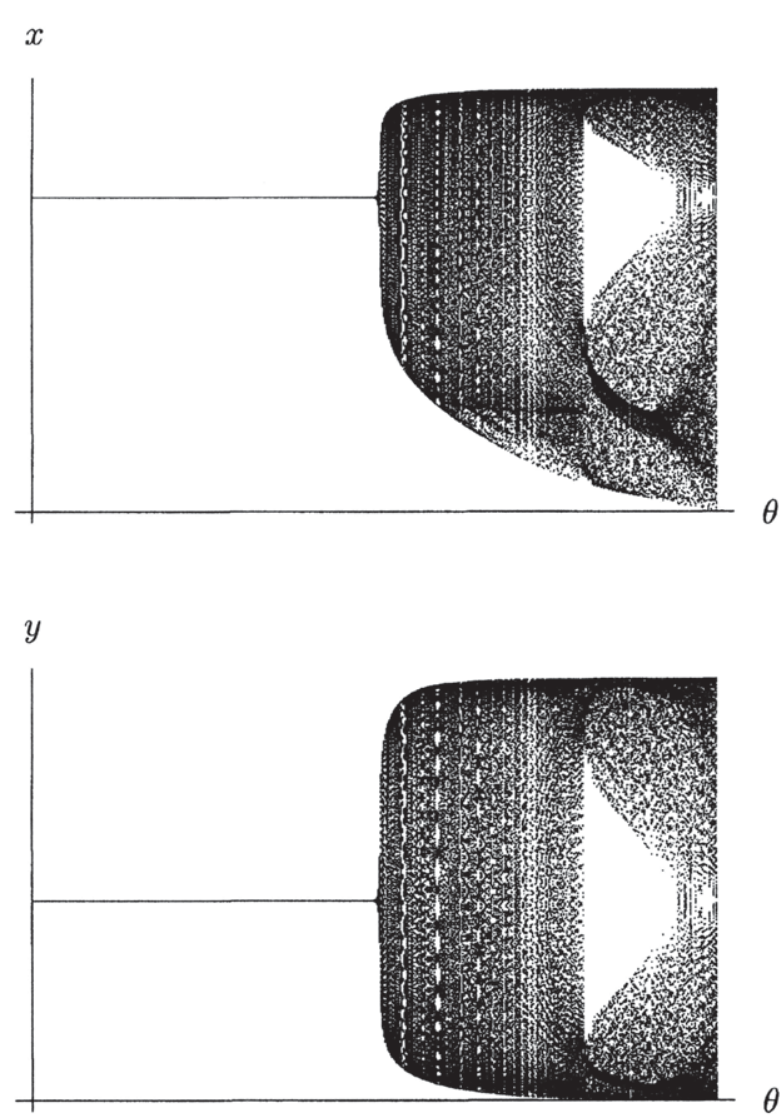

Abbildung 5.29: Feigenbaum-Diagramme des Modells $\mathcal{S}$, zweidimensionale Variante

Fehlentwicklungen entstehen, und der Kosten einer zentralen Planung, wobei Fehlentwicklungen durch strategisches Verhalten der Partner zu berücksichtigen sind.

Einen interessanten Ansatzpunkt, der den Rahmen dieser Arbeit jedoch sprengen würde, bietet in diesem Zusammenhang die Analyse der Gewinnverteilung bei stabilen und chaotischen Regimes. Das Nash-Gleichgewicht stellt zwar eine Win-Win-Situation dar, nicht jedoch ein Gewinnmaximum für die Kooperation als Ganzes. Es ist daher, im Gegensatz zu Modell $\mathcal{V}$, keineswegs klar, ob ein Verfehlen des Nash-Gleichgewichts zu einer Verminderung der 


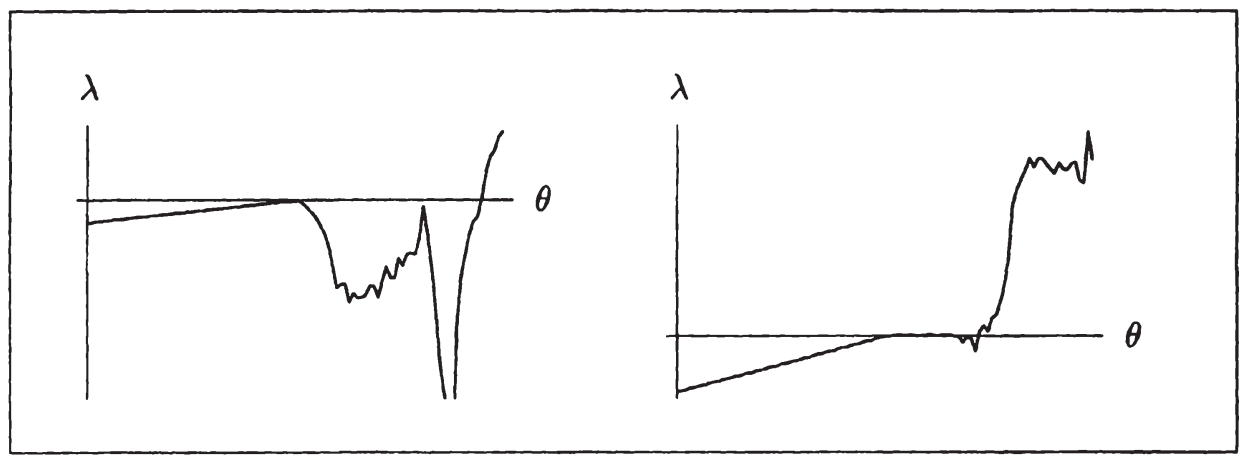

Abbildung 5.30: Lyapunov-Exponenten des Modells $\mathcal{S}$, zweidimensionale Variante

Gewinnsumme $\pi_{x}+\pi_{y}$ führt. Die folgende Übersicht zeigt die Entwicklung der Gewinne absolut und in Prozent für wachsendes $\theta$ :

\begin{tabular}{|c|ll|ll|ll|}
\hline$\theta$ & \multicolumn{2}{|c|}{$\pi_{x}$} & \multicolumn{2}{c|}{$\pi_{y}$} & \multicolumn{2}{c|}{$\pi_{x}+\pi_{y}$} \\
\hline 0,94 & 0,7432 & 100,00 & 0,0190 & 100,00 & 0,7622 & 100,00 \\
0,95 & 0,7420 & 99,85 & 0,0201 & 105,60 & 0,7621 & 99,99 \\
0,96 & 0,7262 & 97,72 & 0,0386 & 203,11 & 0,7649 & 100,35 \\
0,97 & 0,7167 & 96,44 & 0,0506 & 265,91 & 0,7673 & 100,67 \\
0,98 & 0,7056 & 94,95 & 0,0651 & 342,29 & 0,7708 & 101,12 \\
0,99 & 0,7073 & 95,18 & 0,0690 & 362,92 & 0,7764 & 101,86 \\
1 & 0,7077 & 95,22 & 0,0673 & 353,63 & 0,7749 & 101,67 \\
\hline
\end{tabular}

Die Gewinnsumme bleibt nahezu konstant, sie verändert sich um maximal $2 \%$ gegenüber dem Wert im Nash-Gleichgewicht. Für die Schlussfolgerung, das chaotische Regime sei vorzuziehen, weil es zu einer Gewinnsteigerung führe, erscheint diese geringfügige Erhöhung nicht ausreichend. Zudem ist zu bedenken, dass Berechnungen unter der Annahme vollständig linearer Technologien vorgenommen wurden: Die Gewinnfunktionen enthalten keine Fixkosten. Eine Empfehlung für das chaotische Regime lässt sich jedoch nur dann aufrecht erhalten, wenn Kapazitätsanpassungsmaßnahmen bzw. Leerkosten berücksichtigt werden. Unzweideutig ist hingegen der Gewinnumverteilungseffekt des chaotischen Regimes. Der Gewinn des Partners $\mathcal{X}$ sinkt um ca. 0,04, der des Partners $\mathcal{Y}$ steigt um ungefähr denselben Betrag. Relativ gesehen bedeutet dies jedoch lediglich eine Gewinnminderung für $\mathcal{X}$ um $5 \%$, wohingegen der Gewinn von $\mathcal{Y}$ um mehr als das Dreifache steigt. Es muss also davon ausgegangen werden, dass die Partner nicht das gleiche Interesse daran haben, die Kooperation in stabile Bahnen zu lenken. Eine eingehende Analyse der Anreizsituation mit ihren Folgen für die Systemdynamik könnte 
interessante Einsichten zu Tage bringen. An dieser Stelle können wir es mit dem Hinweis bewenden lassen, dass die Machtverhältnisse innerhalb der Kooperation eine entscheidende Rolle spielen und hier die Gewinnverteilung Ergebnis des Verhandlungsprozesses über die Gewichte der Verteilungsfunktionen - ein deutliches Übergewicht von Partner $\mathcal{X}$ andeutet.

\subsection{Zusammenfassung der Ergebnisse}

In diesem Kapitel wurden Modelle zur Koordination zwischen Individuen im Allgemeinen bzw. in Unternehmen im Besonderen vorgestellt. Abschnitt 5.1 befasste sich mit den Ansätzen der klassischen und evolutorischen Spieltheorie. Hier wird Koordination in den Spielregeln dadurch abgebildet, dass die Spieler ihre Auszahlungen dann maximieren, wenn sie sich auf eine bestimmte Strategienkombination einigen können. Diese Modelle haben das Ziel, ein eindeutiges Ergebnis abzuleiten, um Handlungsempfehlungen geben zu können. Die klassische Spieltheorie zeigt, dass unter allgemeinen Annahmen kein eindeutiges Spielergebnis zu finden ist und versucht schärfere Bedingungen anzugeben, mit denen die Zahl der Gleichgewichte reduziert wird, sei es durch Verschärfungen des Gleichgewichtskonzepts, sei es durch einen Katalog von Axiomen.

Die evolutionäre Spieltheorie beruht nicht so sehr auf dem Rationalitätskonzept, sondern baut auf dem Gedanken des survival of the fittest auf. Sie ist daher besser geeignet, dem Weg zum Gleichgewicht mehr Aufmerksamkeit zu widmen. Durch die Verbindung von evolutionsstabilen Gleichgewichten mit einer Anpassungsdynamik kann sie - ohne an die Spieler Rationalitätsforderungen stellen zu müssen - aus Anfangsbedingungen ein eindeutiges Ergebnis der Koordination herleiten.

Beide spieltheoretische Konzepte sind aber auf die Prognose von möglichst stabilen Gleichgewichten ausgerichtet. Sowohl das statische NashGleichgewicht als auch die konvergente Replikator-Dynamik klammern die Möglichkeit chaotischen Verhaltens von vornherein aus. Anders sind hingegen die Modelle des Abschnitts 5.2 konzipiert.

Die Bandbreite in den Modellen $\mathcal{B}, \mathcal{V}$ und $\mathcal{S}$ der analysierten Szenarien zeigt, dass Chaos in zu koordinierenden Systemen in keiner in der Realität existierenden Koordinationsform und bei keinem in der unternehmerischen Praxis verwendeten Koordinationsinstrument prinzipiell ausgeschlossen werden kann. Der Geltungsbereich der Modelle reicht über die analysierten Szenarien hinaus auf die damit konjugierten Systeme. Der Zusammenhang zwi- 
schen den die Dynamik bestimmenden Parametern und dem Systemverhalten gilt hingegen nur für jeweils eine Modellspezifikation. Dies macht die Formulierung von Handlungsempfehlungen sehr schwierig und dürfte das größte Hindernis sein, dass sich der Chaostheorie auf dem Weg zu einem Instrument der Betriebswirtschaftslehre entgegenstellt: die Notwendigkeit, die Analyse anhand numerisch spezifizierter Systeme vorzunehmen, und die Unmöglichkeit, die numerischen Ergebnisse in Handlungsempfehlungen für die betriebliche Praxis umzusetzen.

Die Vielfalt in den Koordinationsmodellen erstreckt sich auf die systembestimmenden Parameter. In Modell $\mathcal{B}$ handelte es sich um eine Entscheidungsvariable der Unternehmensleitung, nämlich die Regel zur Bestimmung der Budgets. Hier haben es die Entscheidungsträger in der Hand, durch eine entsprechende Vorgabe des Budgets die Unternehmensentwicklung zu verstetigen. Sobald die Wirkung dieses Parameters verstanden wird, gibt es kein prinzipielles Problem mehr. Lediglich das genaue Intervall der unkritischen Parameterwerte kann aus dem Modell nicht allgemein bestimmt werden. Das Modell gibt immerhin die Aussage her, dass die kritischen Bereiche bei eher hohen Budgets zu finden sind. Diese Bereiche zu verlassen, sollte der Unternehmensleitung jedoch in vertretbarer Zeitspanne gelingen.

Etwas anders stellte sich die Situation in Modell $\mathcal{V}$ dar. Hier war die Adaptionsgeschwindigkeit im Anpassungsprozess der entscheidende Parameter. Im Gegensatz zu Modell $\mathcal{B}$ liegt dieser Parameter nicht gleichsam auf dem Tisch, sondern ist ein Verhaltensparameter des Entscheidungsträgers, dessen sich dieser womöglich gar nicht bewusst ist. Dieses Modell zeigt besonders die Wichtigkeit auf, sich der dynamischen Wirkung von Parametern bewusst zu werden. Hier kann das Systemverhalten von stabilem zu chaotischem Verhalten wechseln, ohne dass sich dem Anschein nach etwas verändert hat.

Das Modell $\mathcal{S}$ wurde in zwei Varianten diskutiert, um die Interaktion verschiedener Parameter zu beleuchten. Die erste, eindimensionale Variante enthielt als Parameter das Verhältnis der Grenzkosten zweier Unternehmen in Kooperation. Dieser Parameter wird von der Technologie determiniert; es sieht beim ersten Blick so aus, als entziehe sich die Systemdynamik dem Einfluss der Unternehmensleitungen. In der zweiten, zweidimensionalen Variante wurde jedoch als zusätzlicher Parameter die Adaptionsgeschwindigkeit eingeführt und gezeigt, wie sich die Parameter wechselweise beeinflussen. Die Wirkung des Grenzkostenverhältnisses kann durch die Wahl der Adaptionsgeschwindigkeit konterkariert werden; die angemessene Adaptionsgeschwindigkeit hängt aber wiederum vom Grenzkostenverhältnis ab. Die Aussage aus 
Modell $\mathcal{V}$, dass chaotische Bereiche eher bei hoher Adaptionsgeschwindigkeit erreicht werden, wird in diesem Modell bestätigt.

Gegen Modell $\mathcal{S}$ ließe sich einwenden, dass der chaotische Bereich so klein ist, dass er ökonomisch irrelevant oder zumindest von geringer Bedeutung ist. Darauf lässt sich erwidern, dass zum einen die Grenzen des kritischen Bereichs von der Spezifikation abhängt und somit keine Allgemeingültigkeit beanspruchen kann und zum anderen im Rahmen von Modell $\mathcal{B}$ demonstriert wurde, wie stochastische Einflüsse der Sensitivität eines Systems Vorschub leisten. Die Relevanz eines chaotischen Modells lässt sich also nicht danach beurteilen, wie groß der chaotische Bereich der untersuchten Modellspezifikation ist. 


\section{Kapitel 6}

\section{Fazit}

In einem arbeitsteiligen System ist die Frage nach der Koordination der einzelnen Teilleistungen im Hinblick auf die Erfüllung der gesamten Aufgabe von grundsätzlicher Bedeutung. Bislang hat die Forschung das Augenmerk auf die Bedingungen und Instrumente gelegt, die zu erfolgreicher Koordination führen. Sei es die verbal ausgerichtete Organisationstheorie, sei es die formal orientierte Spieltheorie, im Mittelpunkt steht stets die Frage, wie sich ein Zustand charakterisieren lässt, in dem Arbeitsteilung und Zusammenarbeit zu einem gewünschten Ergebnis führen und unter welchen Bedingungen bzw. durch den Einsatz welcher Instrumente sich dieser Zustand erreichen lässt.

Die vorliegende Arbeit ist das Problem der Koordination aus einer chaostheoretischen Perspektive heraus angegangen. Es wurde untersucht, unter welchen Umständen die Anwendung von Koordinationsmechanismen zu chaotischen Schwankungen führt. Es zeigte sich, dass keine Koordinationsform vor Chaos sicher ist, man aber in jedem Fall die systembestimmenden Parameter in stabilen Bereichen halten kann.

Dies ist die Botschaft aller chaostheoretischen Modelle in der Betriebswirtschaftslehre. Berücksichtigt man in einem dynamischen Kontext, dass die Zusammenhänge zwischen den relevanten Größen nichtlinear sind, muss man immer mit dem Auftreten chaotischer Schwingungen rechnen. Es muss ein Bewusstsein dafür bestehen, dass irreguläres Verhalten ökonomischer Systeme nicht nur auf stochastischen Einflüssen beruht, sondern auch in einem deterministischen System immanent auftreten kann, und ein Wissen darüber, wie man mit diesem Phänomen umgehen kann. Dann erst sind die Entscheidungsträger in der Lage, das System aktiv und gezielt zu gestalten, statt sich vermeintlich stochastischen Einflüssen hilflos gegenüber zu sehen. Ihre 
Aufgabe besteht darin, die systembestimmenden Parameter zu identifizieren und die stabilitätsbegünstigenden Wertebereiche in einem Lernprozess $\mathrm{zu}$ ermitteln.

Die in Abschnitt 5.2 vorgestellten Modelle greifen das Problem der Koordination von auf mehrere Schultern verteilten Aufgaben auf und zeigen, dass chaotisches Verhalten des Systems sehr unterschiedliche Ursachen haben kann. Die einen sind plausibel und nahe liegend: Dass eine große Adaptionsgeschwindigkeit zu permanentem Über- und Unterschießen von Systemvariablen führt, kann nicht weiter überraschen. Andere wiederum sind nicht auf den ersten Blick einleuchtend: Dass das Grenzkostenverhältnis über die Systemstabilität entscheidet, würde man im Vorhinein kaum vermuten.

Bis sich aus solchen Modellen mit einiger Sicherheit ein vollständiges Bild chaosauslösender Faktoren in Koordinationsmechanismen ergibt, ist sicherlich noch erhebliche Forschungsarbeit zu leisten. Die vorliegende Arbeit hat hier für ganz unterschiedliche Szenarien Grundmodelle vorgelegt, auf die aufbauend umfassendere Modelle, die zusätzliche Parameter und Systemvariablen berücksichtigen, entwickelt werden können.

Bei der Beurteilung dieser Modelle mag man kritisieren, dass sich die Analyse und Identifikation chaotischer Bereiche auf numerisch spezifizierte Modelle bezieht. Dementsprechend gelten Handlungsempfehlungen zur Vermeidung von Chaos vorderhand nur für eben diese Spezifikationen. Nun sind ökonomische Modelle in der Regel darauf bedacht, möglichst wenig zu spezifizieren. Oft wird nicht nur auf die Bestimmung von Parametern verzichtet, sondern sogar die Form der Modellgleichungen so allgemein wie möglich gehalten. Beispielsweise wird für eine Produktionsfunktion lediglich unterstellt, dass sie neoklassische Eigenschaften hat. Damit wird angenommen, dass sie abnehmende positive Grenzerträge aufweist, eine abnehmende Grenzrate der Substitution besitzt und die Skalenerträge nicht zunehmen. Eine speziellere Form, z.B. eine Cobb-Douglas-Funktion wird höchstens für didaktische Zwecke verwendet, etwa um die allgemeinen neoklassischen Eigenschaften veranschaulichen zu können. Darüber hinaus kann man einwenden, dass nichtlineare Modelle in der Regel auf einer sehr hohen Abstraktionsebene stehen, d.h. nur sehr wenige Gleichungen, Variablen und Parameter enthalten und damit viele reale Interdependenzen vernachlässigen. Betriebswirtschaftliche Modelle werden daher häufig als lineare Optimierungsmodelle formuliert, wobei man sich darum bemüht, die Einflussfaktoren und ihre Interdependenzen möglichst vollständig abzubilden.

Diese Vorgehensweisen sind selbstverständlich sinnvoll: In der Ökonomik ist es nur selten möglich, Sachverhalte strukturgleich durch spezifische Funk- 
tionen abzubilden. In der Regel zwingt die Komplexität der Sachverhalte, die allgegenwärtige Interdependenz zu starker Vereinfachung. Insbesondere menschliches Verhalten entzieht sich dem Schema mathematischer Formeln. Der fehlenden Exaktheit wegen versuchen Ökonomen also, mathematische Modelle so allgemein wie nur irgend möglich zu halten. Denn Erkenntnisse, die auf diese Weise gewonnen werden, können eine dementsprechende Allgemeinheit beanspruchen. Lineare Modelle sind prinzipiell in jedem Umfang lösbar, insbesondere wenn sie auf Ganzzahligkeitsbedingungen verzichten, sogar in kurzer Rechenzeit. Sie sind daher besonders geeignet, Probleme großen Umfangs zu bearbeiten.

Das in der vorliegenden Arbeit gewählte Vorgehen der Verwendung numerischer Spezifikationen ist dennoch wohl begründet. Zunächst muss auf den Nachteil der oben skizzierten Methode verwiesen werden. Die Erkenntnisse aus allgemein gehaltenen Modellen sind oft recht spärlich. So lässt sich aus der neoklassischen Theorie des Haushalts eine inverse Beziehung zwischen dem Marktpreis eines Gutes und der Nachfrage eines Haushaltes nach diesem Gut, d.h. eine fallende Nachfragefunktion, nicht herleiten. Hierzu ist die Annahme nötig, dass der Einkommenseffekt einer Preisänderung den Substitutionseffekt nicht dominiert. Dies ist aber durch das entscheidungslogische Rationalprinzip, das die Basis der neoklassischen Theorie des Haushalts bildet, nicht gedeckt und beispielsweise für den Arbeitsmarkt empirisch widerlegt. Die Nachfrage nach Freizeit müsste nämlich bei steigendem Reallohn zurückgehen, da entgangener Lohn der Preis für Freizeit ist. In der Tat ist aber das Arbeitsangebot in den letzten hundert Jahren bei steigendem Lohn permanent zurückgegangen. Lineare Modelle vernachlässigen den in der Realität vorherrschenden nichtlinearen Charakter ökonomischer Zusammenhänge. Z.B. werden Stückdeckungsbeiträge konstant gesetzt, obwohl weder Preise noch Stückkosten unabhängig von der abgesetzten bzw. hergestellten Menge sein müssen.

Der Nachweis von Chaos in ökonomischen dynamischen Systemen ist nun an die Beschreibung nichtlinearer Systeme durch konkrete Funktionen geknüpft. Es gibt keine Kriterien, die für allgemein gehaltene Funktionen herangezogen werden könnten. Die Definitionen selbst lassen sich nur auf spezifische Funktionen anwenden und darüber hinaus gelingt der Nachweis anhand einer Definition oftmals nicht, so dass man auf numerische Simulationen angewiesen ist, die aber einen mathematischen Beweis nicht ersetzen können, sondern nur zur Aufstellung von Hypothesen taugen.

Eine wichtige Hilfe ist das Konzept der topologischen Konjugation, das im Modell $\mathcal{B}$ explizit herangezogen wurde, aber auch in den anderen Model- 
len seine Gültigkeit und Bedeutung behält. Topologisch konjugierte Systeme besitzen dieselben qualitativen dynamischen Eigenschaften, d.h. der Nachweis von Chaos für ein konkretes System erstreckt sich auf alle konjugierten Systeme. Insofern kann man Modelle mit chaotischer Dynamik niemals als irrelevant weil numerisch spezifiert abtun.

Über die Notwendigkeit, die Erkenntnisse der Theorie nichtlinearer dynamischer Systeme in das Instrumentarium der Betriebswirtschaftslehre einzugliedern, kann kein ernsthafter Zweifel mehr bestehen. Der Chaostheorie kommt dabei nicht die Rolle eines neuen Paradigmas zu, das alte Paradigmen ersetzen müsste. Sondern sie tritt als Methode neben andere Methoden der Mathematik, Natur- und Sozialwissenschaften. Die zukünftige Aufgabe wird sein aufzuzeigen, bei welchen Problemen Vereinfachungen durch Linearisierung oder statische Analyse gerechtfertigt sind und wo neue Erkenntnisse durch Berücksichtigung nichtlinearer Zusammenhänge in dynamischem Kontext zu erwarten sind. 


\section{Literaturverzeichnis}

Albach, Horst (1987). Geburt und Tod von Unternehmen. Techn. Ber. 55, IFM-Materialien

Alchian, Armen A. (1950). Uncertainty, Evolution, and Economic Theory. The Journal of Political Economy, 58, 211-221

Alchian, Armen A.; Demsetz, Harold (1972). Production, Information, Costs and Economic Organization. American Economic Review, 62, 777-795

Alligood, Kathleen T.; Sauer, Tim D.; Yorke, James A. (1997). Chaos An Introduction to Dynamical Systems. Berlin, New York, Heidelberg: Springer

Andersen, David F. (1988). Foreword: Chaos in System Dynamics Models. System Dynamics Review, 4, 3-13

Arrow, Kenneth J. (1986). Rationality of Self and Others in an Economic System. Journal of Business, 59, S385-S399

Arrow, Kenneth J.; Hahn, Frank (1971). General Competitive Analysis. San Francisco: Holden-Day

Bamberg, Günter; Coenenberg, Adolf (1996). Betriebswirtschaftliche Entscheidungslehre. München: Vahlen, 9. Aufl.

Bamberg, Günter; Spremann, Klaus (Hg.) (1987). Agency Theory, Information, and Incentives. Berlin, New York, Heidelberg: Springer

Bandelow, Christoph (1989). Einführung in die Wahrscheinlichkeitstheorie. Mannheim, Wien, Zürich: BI-Wissenschaft, 2. Aufl.

Banks, J.; Brooks, J.; Cairns, G.; Davis, G.; Stacey, P. (1992). On Devaney's Definition of Chaos. American Mathematical Monthly, 99, 332-334 
Barro, Robert J. (1974). Are Government Bonds Net Wealth? Journal of Political Economy, 82, 1095-1117

Barro, Robert J.; Sala-i-Martin, Xavier (1995). Economic Growth. New York: McGraw-Hill

Baumol, William; Benhabib, Jess (1989). Chaos: Significance, Mechanism, and Economic Applications. Journal of Economic Perspectives, 3, 77-105

Becker, Gary S. (1976). The Economic Approach to Human Behavior. Chicago (Ill.): University of Chicago Press

Behrens, D. A.; Feichtinger, G.; Prskawetz, A. (1997). Complex Dynamics and Control of Arms Race. European Journal of Operational Research, $100,192-215$

Benhabib, Jess (Hg.) (1992). Cycles and Chaos in Economic Equilibrium. Princeton, N.J.: Princeton University Press

Benhabib, Jess; Day, Richard H. (1980). Erratic Accumulation. Economics Letters, 6, 113-117

Benhabib, Jess; Day, Richard H. (1981). Rational Choice and Erratic Behaviour. Review of Economic Studies, 48, 459-471

Benhabib, Jess; Day, Richard H. (1982). A Characterization of Erratic Dynamics in the Overlapping Generations Model. Journal of Economic Dynamics and Control, 4, 37-55

Benhabib, Jess; Nishimura, Kazuo (1985). Competitive Equilibrium Cycles. Journal of Economic Theory, 35, 284-306

Bennettin, Giancarlo; Galgani, Luigi; Giorgilli, Antonio; Strelcyn, JeanMarie (1980). Lyapunov Characteristic Exponents for Smooth Dynamical Systems and for Hamiltonian Systems; a Method for Computing All of Them. Meccanica, 15, 9-20

Binmore, Ken (1987). Modelling Rational Players, Part I. Economics and Philosophy, 3, 179-214

Binmore, Ken (1988). Modelling Rational Players, Part II. Economics and Philosophy, 4, 9-55

Blankart, Charles B. (1998). Öffentliche Finanzen in der Demokratie. München: Vahlen, 3. Aufl. 
Boldrin, Michele; Montrucchio, Luigi (1986). On the Indeterminacy of Capital Accumulation Paths. Journal of Economic Theory, 40, 26-39

Brenner, Thomas (1998). Can Evolutionary Algorithms Describe Learning Processes? Journal of Evolutionary Economics, 8, 271-283

Busse von Colbe, Walther; Hammann, Peter; Laßmann, Gert (1992). Betriebswirtschaftstheorie, Bd. 2. Berlin, New York, Heidelberg: Springer, 4. Aufl.

Byrne, John A. (1993). The Virtual Corporation. International Business Week, 8. 2. 1993, $36-40$

Cantner, Uwe; Hanusch, Horst (1997). Evolutorische Ökonomik - Konzeption und Analytik. Das Wirtschaftsstudium, 26, 776-785

Chiang, Alpha C. (1984). Fundamental Methods of Mathematical Economics. Singapore: McGraw-Hill, 3. Aufl.

Coase, Ronald H. (1937). The Nature of the Firm. Economica, 4, 386-405

Coenenberg, Adolf (1999). Kostenrechnung und Kostenanalyse. Landsberg am Lech: moderne industrie, 4. Aufl.

Crawford, Vincent P. (1993a). Introduction: Adaptive Dynamics, Part I. Games and Economic Behavior, 5, 315-319

Crawford, Vincent P. (1993b). Introduction: Adaptive Dynamics, Part II. Games and Economic Behavior, 5, 509-513

Crutchfield, J. P.; Farmer, J. D.; Huberman, B. A. (1982). Fluctuations and Simple Chaotic Dynamics. Physics Reports, 92, 45-82

Dahrendorf, Ralf (1958). Homo Sociologicus: Versuch zur Geschichte, Bedeutung und Kritik der Kategorie der sozialen Rolle. Kölner Zeitschrift für Soziologie und Sozialpsychologie, 10, 178-208

van Damme, Eric (1991). Stability and Perfection of Nash Equilibria. Berlin, New York, Heidelberg: Springer, 2. Aufl.

Darwin, Charles $(1859,1975)$. The Origin of Species by Means of Natural Selection. Harmondsworth: Nachdruck: Penguin Books

Davidow, William H.; Malone, Michael S. (1992). The Virtual Corporation. New York: Harper Collins 
Day, Richard H. (1982). Irregular Growth Cycles. American Economic Review, 72, 406-414

Deneckere, Raymond; Pelikan, Steve (1986). Competitive Chaos. Journal of Economic Theory, 40, 13-25

Deser, Frank (1997). Chaos und Ordnung im Unternehmen. Heidelberg: Physica

Devaney, Robert L. (1989). An Introduction to Chaotic Dynamical Systems. Redwood City: Addison-Wesley, 2. Aufl.

Diamond, P. A. (1965). National Debt in a Neoclassical Growth Model. American Economic Review, 55, 1126-1150

Diamond, Phil (1976). Chaotic Behaviour of Systems of Difference Equations. International Journal of Systems Science, 7, 953-956

Ebeling, Werner (1991). Chaos, Ordnung, Information. Frankfurt a. M., Thun: Deutsch, 2. Aufl.

Eckmann, Jean-Pierre; Ruelle, David (1985). Ergodic Theory of Chaos and Strange Attractors. Review of Modern Physics, 57, 617-656

Elschen, Rainer (1991). Gegenstand und Anwendungsmöglichkeiten der Agency-Theorie. Zeitschrift für betriebswirtschaftliche Forschung, 43, 1002-1012

Elsner, Jürgen (1996). Chaos und Zufall am deutschen Aktienmarkt. Heidelberg: Physica

Erramilli, A.; Forys, L. (1991). Oscillations and Chaos in a Flow Model of a Switching System. IEEE Journal on Selected Areas in Communications, $9,171-178$

Ewert, Ralf; Wagenhofer, Alfred (2000). Interne Unternehmensrechnung. Berlin, New York, Heidelberg: Springer, 4. Aufl.

Falconer, Kenneth (1990). Fractal Geometry, Mathematical Foundations and Applications. New York: Wiley

Farmer, J. Doyne; Ott, Edward; Yorke, James A. (1983). The Dimension of Chaotic Attractors. Physica, 7D, 153-180 
Feichtinger, Gustav; Hommes, Cars; Milik, Alexandra (1997). Chaotic Consumption Pattern in a Simple 2-D Addiction Model. Economic Theory, 10, $147-173$

Feichtinger, Gustav; Kopel, Michael (1993). Chaos in Nonlinear Dynamical Systems Exemplified by an RED Model. European Journal of Operational Research, 68, 145-159

Feichtinger, Gustav; Kopel, Michael (1994). Nichtlineare dynamische Systeme und Chaos: Neue Impulse für die Betriebswirtschaftslehre? Zeitschrift für Betriebswirtschaft, 64, 7-34

Feigenbaum, Mitchell (1978). Quantitative Universality for a Class of Nonlinear Transformations. Journal of Statistical Physics, 19, 25-52

Feigenbaum, Mitchell (1979). The Universal Metric Properties of Nonlinear Transformations. Journal of Statistical Physics, 21, 669-706

Felderer, Bernhard; Homburg, Stefan (1994). Makroökonomik und neue Makroökonomik. Berlin, New York, Heidelberg: Springer, 6. Aufl.

Finkenstädt, Bärbel; Kuhbier, Peter (1992). Chaotic Dynamics in Agricultural Markets. Annals of Operations Research, 37, 73-96

Fischer, Gerd (1995). Lineare Algebra. Braunschweig, Wiesbaden: Vieweg, 10. Aufl.

Forrester, Jay W. (1972). Industrial Dynamics. Cambridge, Mass.: MIT Press

Forster, Otto (1992). Analysis, Bd. 1. Braunschweig, Wiesbaden: Vieweg, 4. Auf.

Forster, Otto (1993). Analysis, Bd. 2. Braunschweig, Wiesbaden: Vieweg, 5. Aufl.

Frederickson, Paul; Kaplan, James L.; Yorke, Ellen D.; Yorke, James A. (1983). The Liapunov Dimension of Strange Attractors. Journal of Differential Equations, 49, 185-207

Frese, Erich (2000). Grundlagen der Organisation. Wiesbaden: Gabler, 8. Aufl.

Friedman, Daniel (1998a). On Economic Applications of Evolutionary Game Theory. Journal of Evolutionary Economics, 8, 15-43 
Friedman, Daniel (1998b). Evolutionary Economics Goes Mainstream: A Review of the Theory of Learning in Games. Journal of Evolutionary Economics, 8, 423-432

Gaertner, W. (1986). Zyklische Konsummuster. Jahrbücher für Nationalökonomie und Statistik, 201, 54-65

Garfield, A.; Spano, M. L.; Ditto, W. L.; Weiss, J. N. (1992). Controlling Cardiac Chaos. Science, 257, 1230-1235

Geist, Karlheinz; Parlitz, Ulrich; Lauterborn, Werner (1990). Comparison of Different Methods for Computing Lyapunov Exponents. Progress of Theoretical Physics, 83, 875-893

Gerthsen, Christian (1997). Physik. Berlin, New York, Heidelberg: Springer, 19. Aufl.

Glass, Leon; Shrier, Alvin; Bélair, Jacques (1986). Chaotic Cardiac Rhythms. In: Holden, Arun V. (Hg.), Chaos. Manchester: Manchester University Press, 237-256

Gleick, James (1988). Chaos - die Ordnung des Universums. München: Droemer Knaur

Grandmont, Jean-Michel (1985). On Endogenous Competitive Business Cycles. Econometrica, 53, 995-1045

Granovetter, Mark; Soong, Roland (1983). Threshold Models of Diffusion and Collective Behavior. Journal of Mathematical Sociology, 9, 165-179

Granovetter, Mark; Soong, Roland (1986). Threshold Models of Interpersonal Effects in Consumer Demand. Journal of Economic Behavior and Organization, 7, 83-99

Guckenheimer, John; Holmes, Philip (1983). Nonlinear Oscillations, Dynamical Systems, and Bifurcations of Vector Fields. Berlin, New York, Heidelberg: Springer

Gutenberg, Erich (1989). Zur Theorie der Unternehmung. Berlin, New York, Heidelberg: Springer. Schriften und Reden von Erich Gutenberg; aus dem Nachlass hg. von Horst Albach

Güth, Werner (1999). Spieltheorie und ökonomische (Bei-)Spiele. Berlin, New York, Heidelberg: Springer, 2. Aufl. 
Güth, Werner; Kalkofen, Brigitte (1989). Unique Solutions for Strategic Games. Berlin, New York, Heidelberg: Springer

Hammerstein, Peter; Selten, Reinhard (1994). Game Theory and Evolutionary Biology. In: Aumann, Robert J.; Hart, Sergiu (Hg.), Handbook of Game Theory, Vol. II. Amsterdam, Lausanne, New York: Elsevier, 929-994

Harsanyi, John C.; Selten, Reinhard (1988). A General Theory of Equilibrium Selection in Games. Cambridge (Mass.): MIT Press

Hart, Sergiu (1992). Games in Extensive and Strategic Forms. In: Aumann, Robert J.; Hart, Sergiu (Hg.), Handbook of Game Theory, Vol. I. Amsterdam, London, New York, Tokyo: North-Holland, 19-40

Hausdorff, Felix (1918). Dimension und äußeres Maß. Mathematische Annalen, $79,157-179$

von Hayek, Friedrich A. $(1969,1994)$. Freiburger Studien - Gesammelte Aufsätze von F.A. von Hayek. Tübingen: Mohr, 2. Aufl.

Hénon, Michel (1976). A Two-dimensional Mapping with a Strange Attractor. Communications in Mathematical Physics, 50, 69-77

Heuser, Harro (1991). Gewöhnliche Differentialgleichungen. Stuttgart: Teubner, 2. Aufl.

Heuser, Harro (1994). Lehrbuch der Analysis, Bd. 1. Stuttgart: Teubner, 11. Aufl.

Hey, John D. (1991). Experiments in Economics. Oxford: Blackwell

Hilbert, David (1891). Über die stetige Abbildung einer Linie auf ein Flächenstück. Mathematische Annalen, 38, 459-460

Hirsch, Morris W.; Smale, Stephen (1974). Differential Equations, Dynamical Systems, and Linear Algebra. Berkeley: Academic Press

Holler, Manfred; Illing, Gerhard (1993). Einführung in die Spieltheorie. Berlin, New York, Heidelberg: Springer, 2. Aufl.

Homburg, Carsten; Schneeweiß, Christoph (1997). Hierarchisch-partizipative Koordinationsprozesse in dezentralen Organisaionen. Zeitschrift für Betriebswirtschaft, 67, 759-779

Hommes, Cars H. (1992). Cobwebs, Chaos and Bifurcations. Annals of Operations Research, 37, 97-100 
Hommes, Cars H. (1994). Dynamics of the Cobweb Model with Adaptive Expectations and Nonlinear Supply and Demand. Journal of Economic Behavior and Organization, 24, 315-335

Hutchinson, John E. (1981). Fractals and Self-Similarity. Indiana University Mathematics Journal, 30, 713-747

van Huyck, John B.; Battalio, Raymond C.; Beil, Richard O. (1990). Tacit Coordination Games, Strategic Uncertainty and Coordination Failure. American Economic Review, 80, 234-248

Jensen, Michael C.; Meckling, William H. (1976). Theory of the Firm: Managerial Behavior, Agency Costs, and Ownership Strucure. Journal of Financial Economics, 3, 305-360

Jetschke, Gottfried (1989). Mathematik der Selbstorganisation. Braunschweig, Wiesbaden: Vieweg

Kakutani, Shizuo (1941). A Generalization of Brouwer's Fixed Point Theorem. Duke Mathematical Journal, 8, 457-459

Kaplan, James L.; Yorke, James A. (1979). Chaotic Behavior of Multidimensional Difference Equations. In: Peitgen, Heinz-Otto; Walter, HansOtto (Hg.), Functional Differential Equations and Approximation of Fixed Points. Heidelberg, Berlin, New York: Springer

Keener, J. P. (1981). Chaotic Cardiac Dynamics. In: Hoppenstaedt, F. C. (Hg.), Mathematical Aspects of Physiology. Providence (R.I.): American Mathematical Society, 299-325

Kelsey, David (1988). The Economics of Chaos or the Chaos of Economics. Oxford Economic Papers, 40, 1-31

Kieser, Alfred; Kubicek, Herbert (1992). Organisation. Berlin, New York: deGruyter, 3. Aufl.

Kirchgässner, Gebhard (2000). Homo Oeconomicus. Tübingen: Mohr, 2. Aufl.

Kistner, Klaus-Peter; Steven, Marion (1993). Produktionsplanung. Heidelberg: Physica, 2. Aufl.

Klír, Jiří; Valach, Miroslav (1967). Cybernetic Modelling. London: Iliffe 
von Koch, Helge (1904). Sur une courbe continue sans tangente, obtenue par une construction géometrique élémentaire. Arkiv för Matematik, 1, 681-704

von Koch, Helge (1906). Une méthode géométrique élémentaire pour l'étude de certaines questions de la théorie des courbes planes. Acta Mathematica, $30,145-174$

Kopel, Michael (1994). Komplexe Unternehmensdynamik: chaotische dynamische Systeme in der Betriebswirtschaftslehre. Wiesbaden: DUV

Kopel, Michael (1996). Kontrolliertes Chaos: Ein Ausweg aus der Unternehmenskrise. Zeitschrift für Betriebswirtschaft, 66, 487-503

Kopel, Michael (1997). Improving the Performance of an Economic System: Controlling Chaos. Journal of Evolutionary Economics, 7, 269-289

Kopel, Michael; Feichtinger, Gustav (1995). Rationalität und komplexes Verhalten. Zeitschrift für betriebswirtschaftliche Forschung, 47, 545-557

Krallmann, Hermann (1996). Systemanalyse im Unternehmen. München, Wien: Oldenbourg, 2. Aufl.

Kreps, David; Wilson, Robert (1982). Sequential Equilibria. Econometrica, 50, 863-894

Kurths, Jürgen; Herzel, H. (1987). An Attractor in a Solar Time Series. Physica D, 25, 165-172

Kuznetsov, Yuri A. (1995). Elements of Applied Bifurcation Theory. Berlin, New York, Heidelberg: Springer

Laux, Helmut; Liermann, Felix (1997). Grundlagen der Organisation. Berlin, New York, Heidelberg: Springer, 4. Aufl.

Li, Tien-Yien; Yorke, James A. (1975). Period Three Implies Chaos. American Mathematical Monthly, 82, 985-992

Loistl, Otto; Betz, Iro (1996). Chaostheorie. Wien, München: Oldenbourg, 3. Aufl.

Lorenz, Edward N. (1963). Deterministic Non-Periodic Flow. Journal of the Atmospheric Sciences, 20, 130-141

Lorenz, Edward N. (1964). The Problem of Deducing the Climate from the Governing Equations. Tellus, 16, 1-11 
Lorenz, Hans-Walter (1993). Nonlinear Dynamical Economics and Chaotic Motion. Berlin, New York, Heidelberg: Springer, 2. Aufl.

Luce, R. Duncan; Raiffa, Howard (1967). Games and Decision. New York: Wiley

Mag, Wolfgang (1990). Grundzüge der Entscheidungstheorie. München: Vahlen

Malthus, Thomas Robert $(1798,1973)$. An Essay on the Principle of Population. London: Nachdruck: Dent

Maly, W. (1982). Zero-Base Budgeting. Zeitschrift für Organisation, 51, 267-268

Mandelbrot, Benoît B. (1967). How Long Is the Coast of Britain? Statistical Self-Similarity and Fractional Dimension. Science, 155, 636-638

Marettek, A. (1982). Arbeitsschritte zur Durchführung der Zero-BaseBudgeting-Analyse. Wirtschaftswissenschaftliches Studium, 11, 257-263

Marotto, Frederick R. (1978). Snap-Back Repellers Imply Chaos in $\mathbb{R}^{n}$. Journal of Mathematical Analysis and Applications, 63, 199-223

Maußner, Alfred (1994). Konjunkturtheorie. Berlin, New York, Heidelberg: Springer

Maußner, Alfred; Klump, Rainer (1996). Wachstumstheorie. Berlin, New York, Heidelberg: Springer

May, Robert M. (1976). Simple Mathematical Models with Very Complicated Dynamics. Nature, 261, 459-467

Maynard Smith, John (1982). Evolution and the Theory of Games. Cambridge (Mass.): Cambridge University Press

Maynard Smith, John; Price, G. R. (1973). The Logic of Animal Conflict. Nature, 246, 15-18

Medio, Alfredo (1992). Chaotic Dynamics. Cambridge (Mass.): Cambridge University Press

Meffert, Heribert (2000). Marketing. Wiesbaden: Gabler, 9. Aufl. 
Metropolis, Nicholas; Stein, Paul; Stein, Myron (1973). On Finite Limit Sets for Transformations on the Unit Interval. Journal of Combinatorial Theory Ser. A, 15, 25-44

Mintzberg, Henry (1979). The Structuring of Organizations. Englewood Cliffs (N.J.): Prentice-Hall

Mushi, Toshimitsu; Higuchi, Hideyo (1976). The $1 / f$ Fluctuation of a Traffic Current on an Expressway. Japanese Journal of Applied Physics, 15, 12711275

Myerson, Roger (1978). Refinements of the Nash Equilibrium Concept. International Journal of Game Theory, 7, 73-80

Nelson, Richard R.; Winter, Sidney G. (1982). An Evolutionary Theory of Economic Change. Cambridge (Mass.), London: Belknap

Peano, Giuseppe (1890). Sur une courbe, qui remplit toute une aire plaine. Mathematische Annalen, 36, 157-160

Peitgen, Heinz-Otto; Jürgens, Hartmut; Saupe, Dietmar (1992). Bausteine des Chaos: Fraktale. Berlin, Heidelberg, New York: Klett-Cotta

Peitgen, Heinz-Otto; Jürgens, Hartmut; Saupe, Dietmar (1994). Chaos: Bausteine der Ordnung. Berlin, Heidelberg, New York: Klett-Cotta

Penrose, Edith Tilton (1952). Biological Analogies in the Theory of the Firm. American Economic Review, 42, 804-819

Perko, Lawrence (1991). Differential Equations and Dynamical Systems. Berlin, New York, Heidelberg: Springer

Pfeiffer, Thomas (1997). Innerbetriebliche Verrechnungspreisbildung bei dezentralen Entscheidungsstrukturen. Heidelberg: Physica

Pies, Ingo (1993). Normative Institutionenökonomik. Tübingen: Mohr

Pinkwart, Andreas (1992). Chaos und Unternehmenskrise. Wiesbaden: Gabler

Poincaré, Henri $(1914,1973)$. Wissenschaft und Methode. Stuttgart: Teubner

Popper, Karl R. $(1945,1992)$. Die offene Gesellschaft und ihre Feinde, Bd. 2. Tübingen: Mohr, 7. Aufl. 
Prigogine, Ilya; Allen, P. M.; Herman, R. (1977). Long Term Trends and the Evolution of Complexity. New York: Pergamon

Prigogine, Ilya; Glansdorff, P. (1971). Thermodynamic Theory of Structure, Stability, and Fluctuations. New York: Wiley

Puu, Tönu (1997). Nonlinear Economic Dynamics. Berlin, New York, Heidelberg: Springer, 4. Aufl.

Rasmusen, Eric (1994). Games and Information. Cambridge (Mass.), Oxford: Blackwell, 2. Aufl.

Rasmussen, Dan; Mosekilde, Erik (1988). Bifurcations and Chaos in a Generic Management Model. European Journal of Operational Research, 35, $80-88$

Rawls, John (1971). A Theory of Justice. Cambrigde (Mass.): Harvard University Press

Rieck, Christian (1993). Spieltheorie. Wiesbaden: Gabler

Ross, Stephen A. (1973). The Economic Theory of Agency: The Principal's Problem. American Economic Review, 63, 134-139

Ruelle, David; Takens, Floris (1971). On the Nature of Turbulence. Communications in Mathematical Physics, 20, 167-192

Samuelson, Paul A. (1948). Consumption Theory in Terms of Revealed Preference. Economica, 15, 243-253

Sandri, Marco (1996). Numerical Calculation of Lyapunov Exponents. Mathematica Journal, 6, 78-84

Saperstein, Alvin M. (1984). Chaos - A Model for the Outbreak of War. Natura, 309, 303-305

Sarkovskii, A. N. (1964). Coexistence of Cycles of a Continuous Mapping of the Line into Itself. Ukrainian Mathematical Journal, 16, 61-71

Schäffer, Utz (1996). Koordination durch Selbstabstimmung. Das Wirtschaftsstudium, 25, 1096-1101

Scheinkman, José (1990). Nonlinearities in Economic Dynamics. The Economic Journal, 100, 33-48 
Schelling, Thomas (1960). The Strategy of Conflict. Cambridge (Mass.): Harvard University Press

Schlicht, Ekkehart (1990). Rationality, Bounded or not, and Institutional Analysis. Journal of Institutional and Theoretical Analysis, 146, 703-719

Schlittgen, Rainer; Streitberg, Bernd (1999). Zeitreihenanalyse. München, Wien: Oldenbourg, 8. Aufl.

Schneider, Dieter (1995a). Betriebswirtschaftslehre, Bd. 1. München, Wien: Oldenburg, 2. Aufl.

Schneider, Dieter (1995b). Informations- und Entscheidungstheorie. München, Wien: Oldenbourg

Schneider, Dieter (1997a). Betriebswirtschaftslehre, Bd. 2. München, Wien: Oldenbourg, 2. Aufl.

Schneider, Dieter (1997b). Betriebswirtschaftslehre, Bd. 3. München, Wien: Oldenbourg

Schumann, Jochen; Meyer, Ulrich; Ströbele, Wolfgang (1999). Grundzüge der mikroökonomischen Theorie. Berlin, New York, Heidelberg: Springer, 7. Aufl.

Schumpeter, Joseph A. (1939). Business Cycles. New York, London: McGraw-Hill

Schweber, Silvan S. (1977). The Origin of the Origin Revisited. Journal of the History of Biology, 10, 229-316

Selten, Reinhard (1965). Spieltheoretische Behandlung eines Oligopolmodells mit Nachfrageträgheit. Zeitschrift für die gesamte Staatswissenschaft, 12, 301-324

Selten, Reinhard (1975). Reexamination of the Perfectness Concept for Equilibrium Points in Extensive Games. International Journal of Game Theory, $4,25-55$

Selten, Reinhard (1991). Evolution, Learning, and Economic Behavior. Games and Economic Behavior, 3, 3-24

Sen, Amartya K. (1997). Rational Fools: A Critique of the Behavioral Foundations of Economic Theory. Philosophy and Public Affairs, 6, 317-344 
Serfling, Klaus (1992). Controlling. Stuttgart: Kohlhammer, 2. Aufl.

Shannon, Claude E. (1948). A Mathematical Theory of Communication. The Bell System Technical Journal, 27, 379-423 und 623-656

Shimada, Ippei; Nagashima, Tomomasa (1979). A Numerical Approach to Ergodic Problems of Dissipative Dynamical Systems. Progress of Theoretical Physics, 61, 1605-1616

Simon, Herbert A. (1955). A Behavioral Model of Rational Choice. Quarterly Journal of Economics, 69, 99-118

Simon, Herbert A. (1978). Rationality as Process and as Product of Thought. American Economic Review, Papers and Proceedings, 68, 1-16

Simon, Herbert A. (1979). Rational Decision Making in Business Organizations. American Economic Review, 69, 493-513

Smale, Stephen (1967). Differentiable Dynamical Systems. The Bulletin of the American Mathematical Society, 74, 747-817

Smale, Stephen (1980). The Mathematics of Time. Berlin, New York, Heidelberg: Springer

Smith, Adam $(1776,1976)$. An Inquiry into the Nature and the Causes of the Wealth of Nations. Oxford: Nachdruck: Clarendon

Solow, Robert M. (1956). A Contribution to the Theory of Growth. Quarterly Journal of Economics, 70, 65-94

Sterman, John D. (1988). Deterministic chaos in models of human behavior: methodological issues and experimental results. System Dynamics Review, $4,148-178$

Sterman, John D. (1989). Deterministic Chaos in an Experimental Economic System. Journal of Economic Behavior and Organization, 12, 1-28

Steven, Marion (1994). Hierarchische Produktionsplanung. Heidelberg: Physica, 2. Aufl.

Steven, Marion (1998). Produktionstheorie. Wiesbaden: Gabler

Steven, Marion; Otterpohl, Lars (2000a). Evolutionäre versus nichtkooperative Spieltheorie. Wirtschaftswissenschaftliches Studium, 29, 201206 
Steven, Marion; Otterpohl, Lars (2000b). Virtuelle Unternehmen aus spieltheoretischer Sicht. In: Albach, Horst; Specht, Dieter; Wildemann, Horst (Hg.), Virtuelle Unternehmen. ZfB-Ergänzungsheft 2/2000, Wiesbaden: Gabler

Strogatz, Steven (1994). Nonlinear Dynamics and Chaos. Reading (Mass.): Perseus

Thoma, Beat (1997). Chaostheorie, Wirtschaft und Börse. München, Wien: Oldenbourg, 2. Aufl.

Thompson, Victor A. (1961). Modern Organization. New York: Knopf

Tomita, K.; Tsuda, I. (1979). Chaos in the Belousov-Zhabotinsky Reaction in a Flow System. Physics Letters, 71A, 489-492

Türschmann, Carl (1990). Stabilität und Chaos in einfachen, dynamischen Modellen der Betriebswirtschaftslehre. Zeitschrift für betriebswirtschaftliche Forschung, 42, 22-49

Tyson, J. J. (1978). On the Appearance of Chaos in a Model of the Belousov Reaction. Journal of Mathematical Biology, 5, 351-362

Varian, Hal R. (1992). Microeconomic Analysis. New York: Norton, 3. Aufl.

Vilks, Arnis; Clausing, Thorsten (1999). Evolutionäre Spieltheorie. Das Wirtschaftsstudium, 28, 1386-1400

Volz, J. (1987). Praktische Probleme des Zero-Base-Budgeting (Gemeinkostenwertanalyse). Zeitschrift für Betriebswirtschaft, 57, 870-881

Warnecke, Hans-Jürgen (1992). Die fraktale Fabrik. Berlin, New York, Heidelberg: Springer

Warnecke, Hans-Jürgen (1993). Revolution der Unternehmenskultur. Berlin, New York, Heidelberg: Springer

Warnecke, Hans-Jürgen (Hg.) (1995). Aufbruch zum Fraktalen Unternehmen. Berlin, New York, Heidelberg: Springer

Weber, Max $(1922,1967)$. Gesammelte Aufsätze zur Wissenschaftslehre. Tübingen: Mohr, 3. Aufl.

Weibler, Jürgen; Deeg, Jürgen (1998). Virtuelle Unternehmen - eine kritische Analyse aus strategischer, struktureller und kultureller Perspektive. Zeitschrift für Planung, 9, 107-124 
Weibull, Jörgen W. (1995). Evolutionary Game Theory. Cambridge (Mass.): MIT Press

Weimann, Joachim (2001). Wirtschaftspolitik: Allokation und kollektive Entscheidung. Berlin, New York, Heidelberg: Springer, 2. Aufl.

Wilding, Richard D. (1998). Chaos Theory: Implications for Supply Chain Management. The International Journal of Logistics Management, 9, 43-56

Wolf, A. (1986). Quantifying Chaos with Lyapunov Exponents. In: Holden, Arun V. (Hg.), Chaos. Manchester: Manchester University Press, 273-290

Zäpfel, Günther; Wasner, Michael (1999). Der Peitschenschlageffekt in der Logistikkette und Möglichkeiten der Überwindung chaotischen Verhaltens. logistik management, 1, 297-309 


\section{Bochumer Beiträge zur Unternehmungsführung und Unternehmensforschung}

Herausgegeben vom Direktorium des Instituts für Unternehmungsführung und Unternehmensforschung der Ruhr-Universität Bochum

Band 1 Busse von Colbe, Walther/Mattessich, Richard (Hrsg.): Der Computer im Dienste der Untemehmungsführung (1968)

Band 2 Busse von Colbe, Walther/Meyer-Dohm, Peter (Hrsg.): Unternehmerische Planung und Entscheidung (1969)

Band 3 Anthony, Robert N.: Harvard-Fälle aus der Praxis des betrieblichen Rechnungswesens. Herausgegeben von Richard V. Mattessich unter Mitarbeit von Klaus Herrnberger und Wolf Lange (1969)

Band 4 Mattessich, Richard: Die wissenschaftlichen Grundlagen des Rechnungswesens (1970)

Band 5 Schweim, Joachim: Integrierte Untemehmungsplanung (1969)

Band 6 Busse von Colbe, Walther (Hrsg.): Das Rechnungswesen als Instrument der Unternehmungsführung (1969)

Band 7 Domsch, Michel: Simultane Personal- und Investitionsplanung im Produktionsbereich (1970)

Band 8 Leunig, Manfred: Die Bilanzierung von Beteiligungen. Eine bilanztheoretische Untersuchung (1970)

Band 9 Franke, Reimund: Betriebsmodelle. Rechensystem für Zwecke der kurzfristigen Planung, Kontrolle und Kalkulation (1972)

Band 10 Wittenbrink, Hartwig: Kurzfristige Erfolgsplanung und Erfolgskontrolle mit Betriebsmodellen (1975)

Band 11 Lutter, Marcus (Hrsg.): Recht und Steuer der internationalen Unternehmensverbindungen (1972)

Band 12 Niebling, Helmut: Kurzfristige Finanzrechnung auf der Grundlage von Kosten- und Erlösmodellen (1973)

Band 13 Perlitz, Manfred: Die Prognose des Unternehmenswachstums aus Jahresabschlüssen deutscher Aktiengesellschaften (1973)

Band 14 Niggemann, Walter: Optimale Informationsprozesse in betriebswirtschaftlichen Entscheidungssituationen (1973)

Band 15 Reichardt, Harald: Der aktienrechtliche Abhängigkeitsbericht unter ökonomischen Aspekten (1974)

Band 16 Backhaus, Klaus: Direktvertrieb in der Investitionsgüterindustrie - Eine Marketing-Entscheiung (1974)

Band 17 Plinke, Wulff: Kapitalsteuerung in Filialbanken (1975)

Band 18 Steffen, Rainer: Produktionsplanung bei Fließbandfertigung (1977)

Band 19 Kolb, Jürgen: Industrielle Erlösrechnung - Grundlagen und Anwendungen (1978)

Band 20 Busse von Colbe, Walther/Lutter, Marcus (Hrsg.): Wirtschaftsprüfung heute: Entwicklung oder Reform? (1977)

Band 21 Uphues, Peter: Unternehmerische Anpassung in der Rezession (1979) 
Band 22 Gebhardt, Günther: Insolvenzprognosen aus aktienrechtlichen Jahresabschlüssen (1980)

Band 23 Domsch, Michel: Systemgestützte Personalarbeit (1980)

Band 24 Schmied, Volker: Alternativen der Arbeitsgestaltung und ihre Bewertung (1982)

Band 25 Wäscher, Gerhard: Innerbetriebliche Standortplanung bei einfacher und mehrfacher Zielsetzung (1982)

Band 26 Weber, Martin: Entscheidungen bei Mehrfachzielen - Verfahren zur Unterstützung von Individual- und Gruppenentscheidungen (1983)

Band 27 Kroesen, Alfred: Instandhaltungsplanung und Betriebsplankostenrechnung (1983)

Band 28 Plinke, Wulf: Erlösplanung im industriellen Anlagengeschäft (1985)

Band 29 Chamoni, Peter: Simulation störanfälliger Systeme (1986)

Band 30 Arning, Andreas: Die wirtschaftliche Bewertung der Zentrenfertigung - Dargestellt am Beispiel einer Fertigungsinsel (1987)

Band 31 Gebhardt, Günther: Finanzielle Planung und Kontrolle bei internationaler Unternehmenstätigkeit

Band 32 Markiewicz, Michael: Ersatzteildisposition im Maschinenbau - Betriebswirtschaftliche Methoden der Planung und Überwachung (1988)

Band 33 Pellens, Bernd: Der Informationswert von Konzernabschlüssen - Eine empirische Untersuchung deutscher Börsengesellschaften (1989)

Band 34 Mrotzek, Rüdiger: Bewertung direkter Auslandsinvestitionen mit Hilfe betrieblicher Investitionskalküle (1989)

Band 35 Deppe, Joachim: Quality Circle und Lernstatt - Ein integrativer Ansatz (1989, 3. Auflage 1993)

Band 36 Rademacher, Michael: Arbeitszeitverkürzung und -flexibilisierung - Formen und betriebliche Auswirkungen (1990)

Band 37 Kaiser, Klaus: Kosten- und Leistungsrechung bei automatisierter Produktion (1991, 2. Auflage 1993)

Band 38 Müller, Hermann: Industrielle Abfallbewältigung - Entscheidungsprobleme aus betriebswirtschaftlicher Sicht (1991)

Band 39 Schörner, Peter: Gesetzliches Insiderhandelsverbot - Eine ordnungspolitische Analyse (1991)

Band 40 Bentler, Martin: Grundsätze ordnungsmäßiger Bilanzierung für die Equitymethode (1991)

Band 41 Brüggerhoff, Jürgen: Management von Desinvestitionen (1992)

Band 42 Bröker, Erich W.: Erfolgsrechnung im industriellen Anlagengeschäft - Ein dynamischer Ansatz auf Zahlungsbasis - (1993)

Band 43 Frankenberg, Peter: Transnationale Analyse US-amerikanischer und deutscher Jahresabschlüsse - Eine theoretische und empirische Untersuchung (1993)

Band 44 Kleinaltenkamp, Michael: Standardisierung und Marktprozeß - Entwicklungen und Auswirkungen im CIM-Bereich (1993)

Band 45 Pellens, Bernhard: Aktionärsschutz im Konzern - Empirische und theoretische Analyse der Reformvorschläge der Konzernverfassung (1994)

Band 46 Reckenfelderbäumer, Martin: Marketing-Accounting im Dienstleistungsbereich - Konzeption eines prozeßkostengestützten Instrumentariums (1995) 
Band 47 Knittel, Friedrich: Technikgestützte Kommunikation und Kooperation im Büro. Entwicklungshindernisse - Einsatzstrategien - Gestaltungskonzepte (1995)

Band 48 Riezler, Stephan: Lebenszyklusrechnung - Instrument des Controlling strategischer Projekte (1996)

Band 49 Schulte, Jörn: Rechnungslegung und Aktienkursentwicklung - Erklärung und Prognose von Aktienrenditen durch Einzel- und Konzernabschlußdaten (1996)

Band 50 Muhr, Martin: Zeitsparmodelle in der Industrie - Grundlagen und betriebswirtschaftliche Bedeutung mehrjähriger Aßeitszeitkonten (1996)

Band 51 Brotte, Jörg: US-amerikanische und deutsche Geschäftsberichte. Notwendigkeit, Regulierung und Praxis jahresabschlußergänzender Informationen (1997)

Band 52 Gersch, Martin: Vernetzte Geschäftsbeziehungen. Die Nutzung von EDI als Instrument des Geschäftsbeziehungsmanagement (1998)

Band 53 Währisch, Michael: Kostenrechnungspraxis in der deutschen Industrie. Eine empirische Studie (1998)

Band 54 Völkner, Peer: Modellbasierte Planung von Geschäftsprozeßabläufen (1998)

Band 55 Fülbier, Rolf Uwe: Regulierung der Ad-hoc-Publizität. Ein Beitrag zur ökonomischen Analyse des Rechts (1998)

\section{Band 1 - 55 erschienen belm Gabler Verlag Wlesbaden}

Band 56 Ane-Kristin Reif-Mosel: Computergestützte Kooperation im Büro. Gestaltung unter Berücksichtigung der Elemente Aufgabe, Struktur, Technik und Personal (2000)

Band 57 Claude Tomaszewski: Bewertung strategischer Flexibilität beim Unternehmensenwerb. Der Wertbeitrag von Realoptionen (2000)

Band 58 Thomas Erler: Business Objects als Gestaltungskonzept strategischer Informationssystemplanung (2000)

Band 59 Joachim Gassen: Datenbankgestützte Rechnungslegungspublizität. Ein Beitrag zur Evolution der Rechnungslegung (2000)

Band 60 Frauke Streubel: Organisatorische Gestaltung und Informationsmanagement in der lernenden Unternehmung. Bausteine eines Managementkonzeptes organisationalen Lernens (2000)

Band 61 Andreas von der Gathen: Marken in Jahresabschluß und Lagebericht. 2001.

Band 62 Lars Otterpohl: Koordination in nichtlinearen dynamischen Systemen. 2002. 


\author{
Peter M. Schulze
}

\title{
Regionales Wachstum und Strukturwandel
}

\section{Quantitative Analysen mit Regionaldaten für die Bundesrepublik Deutschland Unter Mitarbeit von Christoph Balz}

Frankfurt/M., Berlin, Bern, Bruxelles, New York, Oxford, Wien, 2001. XIV, 112 S., zahlr. Abb. und Tab.

Schriften zur empirischen Wirtschaftsforschung. Herausgegeben von Peter M. Schulze. Bd. 4

ISBN 3-631-37787-8 · br. € 25.10*

Durch das Zusammenwachsen Europas und die Wiedervereinigung Deutschlands stellen sich Fragen der Konvergenz und Anteilsveränderung der primären, sekundären und tertiären Wirtschaftsbereiche besonders dringlich, gerade in Hinblick auf Output, Beschäftigung und die Arbeitsproduktivität auf regionaler Ebene.

Hierzu werden für die genannten Größen quantitative Analysen mit Hilfe statistisch-ökonometrischer Verfahren durchgeführt, die theoretische Elemente der Regionalwirtschaftslehre einbeziehen. Diese Untersuchungen basieren auf Bundesländer- und Kreisdaten.

Aus dem Inhalt: Steigende Skalenerträge und regionales Wachstum - Rolle der Dienstleistungen für das regionale Wachstum - Regionale Konvergenz · Nationale, regionale und sektorale Einflüsse auf Output, Beschäftigung und Produktivität · Regionale Wirtschaftsstruktur und regionales Wachstum

Frankfurt/M - Berlin - Bern - Bruxelles - New York - Oxford · Wien

Auslieferung: Verlag Peter Lang AG

Jupiterstr. 15, CH-3000 Bern 15

Telefax (004131) 9402131

*inklusive der in Deutschland gültigen Mehrwertsteuer

Preisänderungen vorbehalten

Homepage http://wuw.peterlang.de 